\title{
MESERAN Calibration for Low Level Organic Residues
}

Federal Manufacturing \& Technologies

M. G. Benkovich

\section{KCP-613-6792}

Published April 2004

Final Report

Approved for public release; distribution is unlimited.

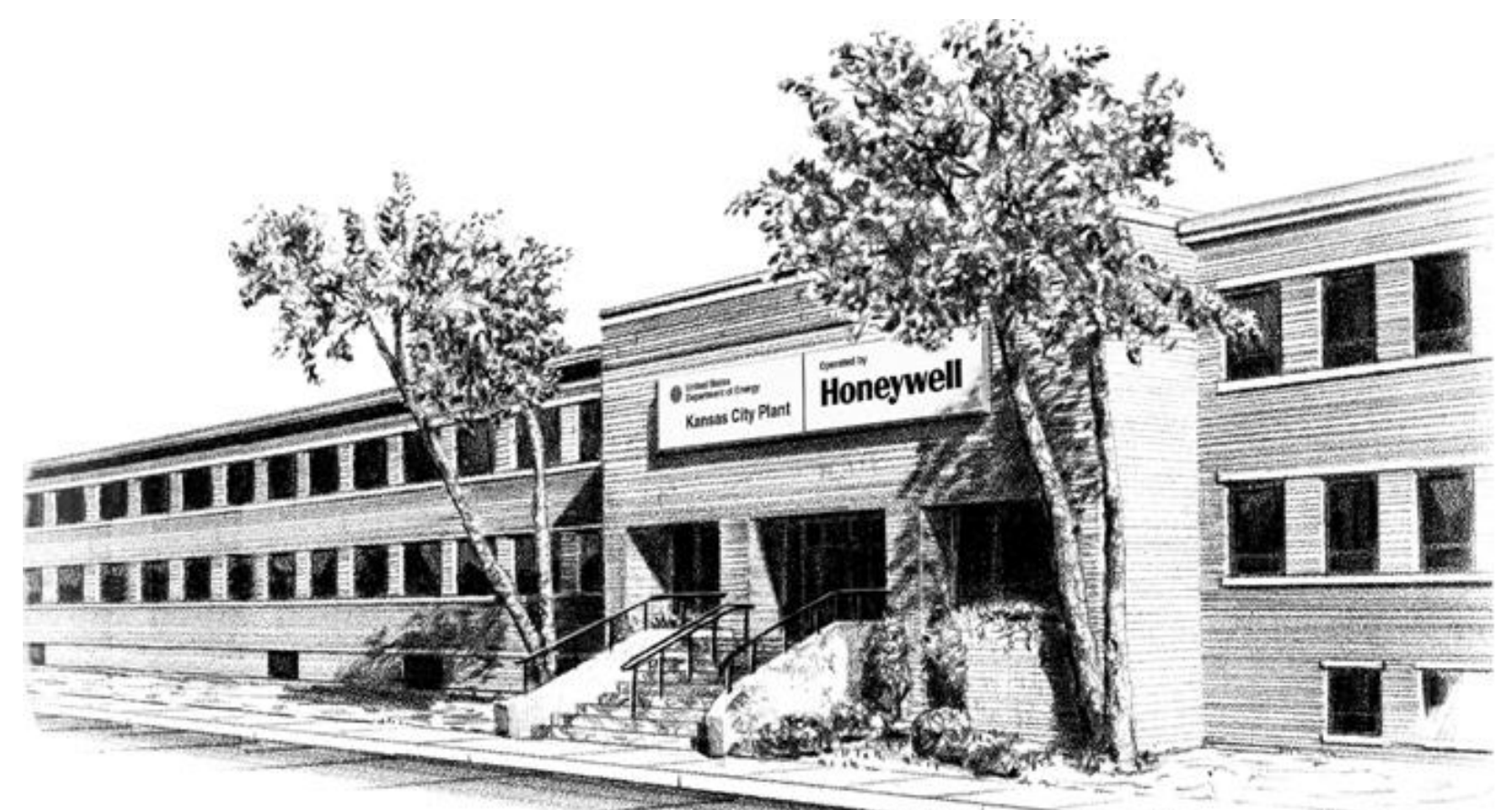




\section{United States Department of Energy}

\section{DISCLAIMER}

This report was prepared as an account of work sponsored by an agency of the United States Government. Neither the United States Government nor any agency thereof, nor any of their employees, nor any of their contractors, subcontractors or their employees, makes any warranty, express or implied, or assumes any legal liability or responsibility for the accuracy, completeness, or any third party's use or the results of such use of any information, apparatus, product, or process disclosed, or represents that its use would not infringe privately owned rights. Reference herein to any specific commercial product, process, or service by trade names, trademark, manufacturer, or otherwise, does not necessarily constitute or imply its endorsement, recommendation, or favoring by the United States Government or any agency thereof or its contractors or subcontractors. The views and opinions of authors expressed herein do not necessarily state or reflect those of the United States Government or any agency thereof.

All data prepared, analyzed and presented has been developed in a specific context of work and was prepared for internal evaluation and use pursuant to that work authorized under the referenced contract. Reference herein to any specific commercial product, process or service by trade name, trademark, manufacturer, or otherwise, does not necessarily constitute or imply its endorsement, recommendation, or favoring by the United States Government, any agency thereof or Honeywell Federal Manufacturing \& Technologies, LLC.

Printed in the United States of America.

This report has been reproduced from the best available copy.

Available to DOE and DOE contractors from the Office of Scientific and Technical Information,

P. O. Box 62, Oak Ridge, Tennessee 37831; prices available from (865) 576-8401,

FTS 626-8401 Facsimile: (865) 576-5728, E-mail: reports@adonis.osti.gov

Available to the public from the National Technical Information Service, U. S. Department of Commerce, 5285 Port Royal Rd., Springfield, Virginia 22161, (800) 553-6847, Facsimile:

(703) 605-6900, E-mail: orders@ntis.fedworld.gov

A prime contractor with the United States Department of Energy under prime contract DE-ACO4-01AL66850.

\section{Honeywell}

Federal Manufacturing

\& Technologies

P. O. Box 419159

Kansas City, Missouri

$64141-6159$ 


\section{MESERAN CALIBRATION FOR LOW LEVEL ORGANIC RESIDUES}

M. G. Benkovich

Published April 2004

Final Report

M. G. Benkovich, Project Leader

Project Team:

G. W. Christoff

T. E. Hand

M. D. Smith

E. T. Walsh II

C. M. Woodburn

John Anderson, ERA Systems, Inc., The MESERAN Company

\section{Contents}

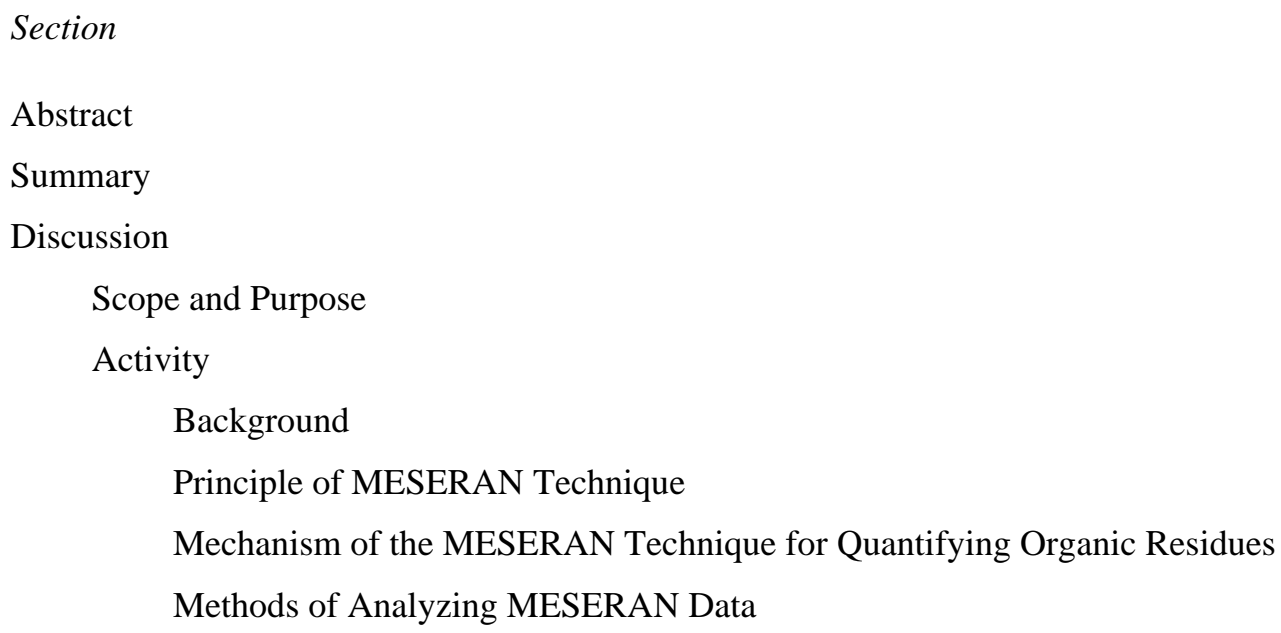


Formulation of Calibration Solutions

Contaminants

Substrates

Cleaning Processes

Calibration Results

Accomplishments

References

Appendices

A. Calibration Curves for Kester 135 Rosin Flux on Aluminum Panels and Stainless Steel Disks

B. Calibration Curves for Kester 185 Rosin Flux on Aluminum Panels and Stainless Steel Disks

C. Calibration Curves for Kester 197 Rosin Flux on Aluminum Panels and Stainless Steel Disks

D. Calibration Curves for Kester 1544 Rosin Flux on Aluminum Panels and Stainless Steel Disks

E. Calibration Curves for WD-40 Oil on Aluminum Panels and Stainless Steel Disks

F. Calibration Curves for Apiezon Grease M on Aluminum Panels and Stainless Steel Disks

G. Calibration Curves for Starrett M-1 Lubricant on Aluminum Panels and Stainless Steel Disks

H. Calibration Curves for Vaseline on Aluminum Panels and Stainless Steel Disks

I. Calibration Curves for Dioctyl Phthalate (DOP) on Aluminum Panels and Stainless Steel Disks

J. Calibration Curves for Carnauba Wax on Aluminum Panels and Stainless Steel Disks

K. Calibration Curves for OSR Mold Release on Aluminum Panels and Stainless Steel Disks

L. Calibration Curves for Thalco 550 Mold Release on Aluminum Panels and Stainless Steel Disks

M. Calibration Curves for RAM 225 Mold Release on Aluminum Panels and Stainless Steel Disks

N. Calibration Curves for Krylon Clear Covercoat Spray on Aluminum Panels and Stainless Steel Disks

O. Calibration Curves for EPON 828 Resin on Aluminum Panels and 
Stainless Steel Disks

P. Calibration Curves for Versamid 140 Curing Agent on Aluminum Panels and Stainless Steel Disks

Q. Calibration Curves for Velva Sheen Mop Oil on Aluminum Panels and Stainless Steel Disks

R. Calibration Curves for Regal R\&O 32 Oil on Aluminum Panels and Stainless Steel Disks

S. Calibration Curves for Hydrocarbon Blend on Aluminum Panels and Stainless Steel Disks

T. Calibration Curves for Mineral Oil on Stainless Steel Disks

U. Calibration Curves for Hexatriacontane on Stainless Steel Disks

\section{Illustrations}

Figure

1 Application of Test Solution

2 Measurement of Emissions

3 Typical Evaporation of Radiochemical Solution From a Clean Surface

4 Typical Evaporations of Radiochemical Solution With Increasing Amounts of Residue

5 Plot of Raw Data Showing Low, Medium, and High Levels of Organic Contamination

6 Logarithm Plot of Smoothed Data Showing Low, Medium, and High Levels of Organic Contamination

7 Calibration Solution Formulation

8 Total Counts Calibration Curve for DOP on Aluminum Panels

9 Low Variance Slope Calibration Curve for DOP on Aluminum Panels 


\begin{abstract}
Precision cleaning studies done at Honeywell Federal Manufacturing \& Technologies (FM\&T), the Kansas City Plant (KCP), and at other locations within the Department of Energy (DOE) Weapons complex over the last 30 years have depended upon results from MESERAN Evaporative Rate Analysis for detecting low levels of organic contamination. The characterization of the surface being analyzed is carried out by depositing a Carbon-14 tagged radiochemical onto the test surface and monitoring the rate at which the radiochemical disappears from the surface with a Geiger-Mueller counter. In the past, the total number of counts over a 2-minute span have been used to judge whether a surface is contaminated or not and semi-quantitatively to what extent. This technique is very sensitive but has not enjoyed the broad acceptance of a purely quantitative analysis. The work on this project developed calibrations of various organic contaminants typically encountered in KCP operations. In addition, a new analysis method was developed to enhance the ability of MESERAN Analyzers to detect organic contamination and yield quantitative data in the microgram and nanogram levels.
\end{abstract}

\title{
Summary
}

Precision cleaning studies done at Honeywell Federal Manufacturing \& Technologies (FM\&T), the Kansas City Plant (KCP), and at other locations within the Department of Energy (DOE) Weapons complex over the last 30 years have depended upon results from MESERAN Evaporative Rate Analysis for detecting low levels of organic contamination. The characterization of the surface being analyzed is carried out by depositing a Carbon-14 tagged radiochemical onto the test surface and monitoring the rate at which the radiochemical disappears from the surface with a Geiger-Mueller counter. In the past, the total number of counts over a 2-minute span have been used to judge whether a surface is contaminated or not and semi-quantitatively to what extent. This technique is very sensitive but has not enjoyed the broad acceptance of a purely quantitative analysis. The purpose of this work is to develop calibrations of various organic contaminants typically encountered in KCP operations and develop an analysis method to enhance the ability of MESERAN Analyzers to detect organic contamination and yield quantitative data in the microgram and possibly nanogram levels.

Calibrations of various organic contaminants typically encountered in KCP operations were developed for aluminum and stainless steel substrates.

A new analysis method was developed to enhance the ability of MESERAN Analyzers to detect organic contamination and yield quantitative data in the microgram and nanogram levels. The MESERAN program has been updated to calculate the various slope methods developed on this project. In addition, a program was developed to convert MESERAN data taken on KCP's automatic MESERAN Analyzers (i.e., various cleaning-related evaluations since 1985) into data that can be analyzed by the various slope methods.

Numerous evaluations were performed to identify "clean" substrates. It was shown that there is more variability in high precision cleaning processes than expected. The MESERAN technique for analyzing cleanliness using the newly developed slope method of analysis was able to discern differences in cleaning processes at low levels of contamination previously not known to exist. Replicate samples cleaned in the exact same processes at the same times are not necessarily cleaned to the same levels of cleanliness. In fact, multiple tests in different areas on the same substrate show varying levels of cleanliness (in the submonolayer region - below about 200 ng).

Plasma cleaning yields cleaner surfaces than wet chemical cleaning alone. Previously it was thought that plasma-cleaned surfaces could not be tested with the MESERAN Analyzer because the activity generated on the surfaces by plasma cleaning would interfere with the evaporation of the radiochemical. Tests performed previously on parts immediately after plasma cleaning showed an increase in total counts because the radiochemical was retained by the active surface. Evaluations were conducted on this project file:///C/ManualConversion/HTML/6792benk.htm (6 of 250)4/9/2004 4:05:24 AM 
that determined that aluminum panels could be successfully evaluated with MESERAN Analysis if they were tested using radiochemical BK (used for detecting nonpolar residues) at least 24 hours after plasma cleaning. Because plasma cleaning activates the surface by making it more polar, the duration of time between plasma cleaning and testing using radiochemical AK (used for detecting polar residues) must be longer. The exact amount of time has not been determined. These discoveries opened up MESERAN testing to plasma-cleaned parts and proved that plasma cleaning does improve the cleanliness of parts.

Surface roughness was found to be a greater-than-expected factor in MESERAN testing. Consistent surfaces are needed to perform accurate calibrations. In addition, calibrations for a particular contaminant do vary on different substrates. Anyone wanting quantifiable contamination data on a particular substrate using direct testing will need to have calibrations performed on that substrate to ensure differences in surface roughness can be eliminated.

Stainless steel disks have been designed with specified machine grooves that allow consistent MESERAN results to be obtained. These disks can be used as reference surfaces for evaluating cleaning processes and cleanliness levels of various products. Parts being analyzed can be extracted with a solvent to remove any potential organic contamination, and the solvent extract can then be evaporated using the MicroSolventEvaporator (developed on a Cooperative Research and Development Agreement - CRADA) to deposit the extracted residue on the disks. The disks can then be tested with the MESERAN Analyzer and the data compared to the calibrations performed on this project (or additional calibrations for specific contaminants of interest) so quantifiable contamination amounts can be determined.

Many contaminants have very similar calibration curves for the same contamination levels. Hydrocarbons tend to test very similarly. However, there are differences that exist in the responses for the wide variety of contaminants evaluated on this project. It may be possible to develop a calibration curve for a general hydrocarbon contaminant for a particular surface; however, it will be much more difficult to develop a general calibration curve for all contaminants.

During the course of the project, problems were encountered with the radiochemical solutions. Some radiochemical solutions appeared to be contaminated and therefore did not test well on clean substrates or low levels of contamination. The new Slope method of analysis was able to detect this problem even though the Total Count method did not. KCP utilized the Six Sigma Process and performed tests that showed that some of the radiochemical solutions The MESERAN Company received from their supplier were contaminated. In addition, The MESERAN Company depleted their supply of radiochemicals and had to reorder new tetrabromoethane-C14 (radiochemical A) and tridecane-C14 (radiochemical B). The newly synthesized tridecane-C14/cyclopentane (BK) did not test the same as the BK used in previously performed calibrations. The tetrabromoethaneC14 also appears to potentially have shelf life issues. Some of the bromine may evolve over time in the concentrated ampules The MESERAN Company receives from its supplier. This results in a mixture of materials instead of just pure tetrabromoethane-C14. Again, the Slope method of analysis detects this whereas the Total Count method does not. Great care must be taken to ensure that each lot of radiochemical solution is identical for the Slope method to be used without performing calibrations for each new lot of radiochemical.

The technology developed on this project has already been used to support KCP as follows:

- Obtain new business for testing NVRs for Lawrence Livermore National Laboratory (LLNL) on the NIF project;

- Evaluate KCP's ability to clean PEPC LRU NIF hardware for LLNL in support of a \$1.2-million reimbursable order;

- Evaluate contamination levels on LAC Shells;

- Evaluate contamination for LED Bond Analysis project in Telemetry;

- Evaluate contamination levels on the Detector Hook Assembly;

- SMRI support for ultrasonic cleaning equipment;

- Evaluate cleaning ability of various atmospheric plasma equipment for Advanced Design and Production Technologies (ADAPT) projects.

Additional work currently scheduled that will utilize the technology developed on this project includes:

- Cleaning evaluations performed for Sandia National Laboratory/New Mexico and Pantex to evaluate alternate wiping solvents;

- Evaluate the ability of plasma cleaning to remove organic contamination from pits for Los Alamos. 


\section{Discussion}

\section{Scope and Purpose}

The purpose of this work is to develop calibrations of various organic contaminants typically encountered in Honeywell Federal Manufacturing \& Technologies (FM\&T), Kansas City Plant (KCP), operations and develop an analysis method to enhance the ability of MESERAN Analyzers to detect organic contamination and yield quantitative data in the microgram and possibly nanogram levels.

\section{Activity}

\section{Background}

The principle of the MESERAN technique was discovered by John L. Anderson, President of ERA Systems, Inc., The MESERAN Company, in 1960. This analytical technique is used in a number of industrial and governmental facilities (within the United States and abroad) for research and development purposes as well as for quality and production control. The characterization of the surface being analyzed is carried out by depositing a chemical detector onto the test surface and observing the rate at which the chemical detector disappears from the surface. The MESERAN technique is routinely used for quantifying organic contamination on surfaces and the crosslink density (or degree of cure) in polymers. In addition, the MESERAN technique can be used for quantifying chemically active sites on surfaces, [1-3].

The Kansas City Plant has been using MESERAN Analyzers for approximately 30 years to detect and quantify organic contamination on parts and evaluate various cleaning processes for removing organic contamination. The KCP has used MESERAN Analyzers extensively to evaluate the ability of alternate solvents and processes for removing specific organic contaminants to eliminate the use of chlorinated and fluorinated solvents, [4-9]. In recent years, the KCP has been working on several projects with The MESERAN Company to improve data analysis and develop new methods for using the MESERAN technology [10-13].

\section{Principle of MESERAN Technique [14]}

The standard microcomputer-based MESERAN technology involves deposition, using a "clean" precision microsyringe, onto a flat or concave surface of $18 \mu \mathrm{L}$ of a test solution consisting of a low boiling solvent or solvent combination (for these evaluations - cyclopentane) and a high-boiling-but-volatile Carbon-14 labeled compound (in a ratio of approximately 60,000:1).

Figure 1 shows the application of test solution. For example, the amount of tridecane-C14 radiochemical per single test $(<0.06 \mu \mathrm{Ci})$ corresponds to approximately $6 \mathrm{E} 14(6 \mathrm{x}$ $10^{14}$ ) molecules which equates to one nanomole, the equivalent of approximately one molecular layer over one square centimeter. Metered air or nitrogen gas is permitted to flow across the surface 
and between the surface and a Geiger Müller detector positioned directly above the surface. The evaporation of the low-boiling solvent and then the radiochemical is observed as a function of time by recording the detected emissions per second arising from the radiochemical molecules remaining on, or retained by, the surface--the vapor-phase, alreadyevaporated molecules having been swept out from under the detector by the metered gas (see Figure 2).

Each test takes less than 3 minutes and the amount of radiochemical employed is EXEMPT from U S Nuclear Regulatory Commission and/or 'Agreement State' licensing regulations due to the very low level of C-14 involved.

For the measurement of microorganic residues, the MESERAN method may be used:

(1) Directly on a flat or concave surface and any microorganic residue thereon which is chemically compatible with the particular radiochemical employed, or

(2) Indirectly using an extracting solvent followed by depositing and evaporating an aliquot amount onto a "clean" reference surface. Subsequent deposition and evaporation of the radiochemical solution permits measurement of the amount of deposited residue by comparing the results with previously obtained standards similarly deposited from volumetric dilutions.

For nonpolar and/or hydrocarbon type residues, tridecane-C14 in cyclopentane (designated BK) is employed. For more polar residues, tetrabromoethane-C14 in cyclopentane (designated $\mathbf{A K}$ ) is used. In order to provide a high number of detected emissions for the minimal amount of radiochemical deposited, the tridecane-C14 has a specific activity of approximately $57 \mu \mathrm{Ci} / \mu$ mole (one tridecane atom is essentially pure $\mathrm{C} 14$ isotope) while the tetrabromoethane has both carbon atoms labeled (approximately $114 \mu \mathrm{Ci} / \mu \mathrm{mole}$ ). Approximately $200 \mathrm{ng}$ of radiochemical are deposited in each test with similar levels of radioactivity.

The MESERAN method assumes that the particular radiochemical employed is chemically compatible with the residue, that the test solution droplet covers all of the residue, and that the test solution solvent substantially dissolves the residue within the time period of the solvent evaporation. Attention to the avoidance of inadvertent contamination and the maintenance of reasonably constant temperature and pressure are required for optimal reproducibility from test to test.

\section{Mechanism of the MESERAN Technique for Quantifying Organic Residues [1-3, 10-13]}

When a homogeneous chemical is permitted to evaporate, the classical mechanism of the process (normally measured by monitoring the already evaporated portion) follows firstorder kinetics, i.e., the plot of $\log$ concentration vs. time is a straight line. This mechanism applies to pure materials as well as to solutions of chemicals in which the components are chemically compatible and in which the second component is nonvolatile under the conditions of the process. In the presence of the second component, the rate of evaporation is slowed.

In the MESERAN technology, however, the amount of radiochemical retained by the surface as a function of time is measured by counting the emissions arising from the radiochemical molecules remaining on the surface. In this discussion, the temperature and pressure are assumed constant and the concentration of already evaporated molecules in the adjacent gaseous phase approaches zero due to the flowing air or nitrogen referred to above. The molecular weight of each evaporating molecule and the intermolecular forces among the near-neighbor molecules are thus the primary factors in determining the tendency of each molecule to remain in solution or conversely to escape from the liquid portion of the air/liquid (or semisolid) interface. In the MESERAN technology, which employs only a monolayer equivalent of the radiochemical, the observed rate of evaporation is thus a function of the residual concentration of the non-evaporated molecules of the Carbon-14 radiochemical. Figure 3 illustrates the typical evaporation of the radiochemical solution from a clean surface. The A-B line represents the evaporation of the low boiling solvent (e.g., cyclopentane). The rationale for the initial increase in counts/second is that the C14 soft beta emissions are partially absorbed by the solvent molecules. B represents the point at which substantially all of the low-boiling solvent has evaporated and the maximal amount of residual radiation reaches the GM detector. The B-C line represents the evaporation of the radiochemical from the surface under the conditions of the test. C represents a level where the GM detector can no longer adequately differentiate the residual radiation from background. 


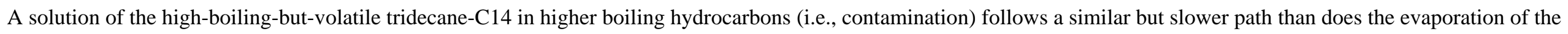

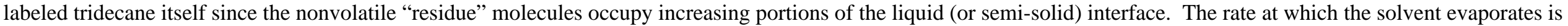

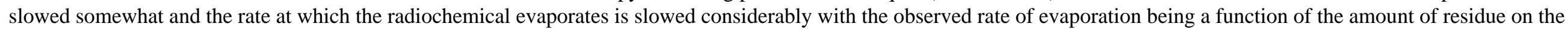

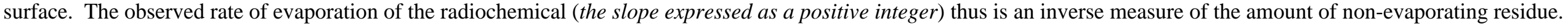

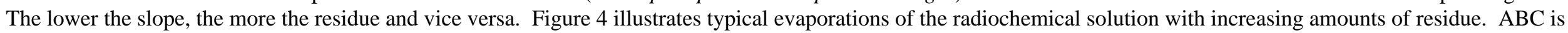

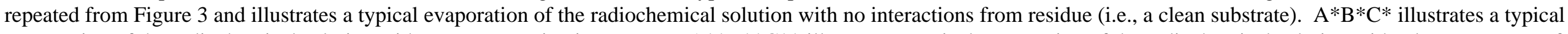

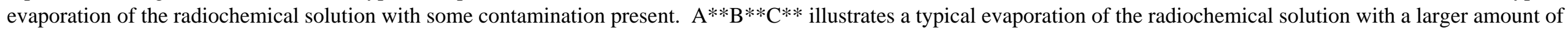
contamination present.

\section{Methods of Analyzing MESERAN Data [1-14]}

\section{Historical Method (Total Counts)}

Prior to the advent of this project, the Kansas City Plant only used the Total Counts method of analysis for analyzing MESERAN data. This involved summing the radioactive counts detected by the GM detector for a period of 112 seconds. The Total Counts method has been used for approximately 30 years at the KCP by testing a surface with the MESERAN Analyzer and comparing the results to those obtained from known clean standards for that particular surface. The total counts of the clean standard are subtracted from the total counts obtained on the surface being tested to give a net total count representing the contamination amount. Lower total counts represent low levels of organic residue and high total counts represent high levels of organic contamination. This result can be compared to previously performed calibrations of contamination to obtain a quantitative result for the contamination amount.

In many cases, quantitative contamination data are not needed. For instance, if one is performing process control work to determine if the cleaning process is performing as designed, quantitative data on the actual amount of contamination may not be necessary. Often times, as long as the parts being cleaned are less than a certain level of contamination, they are clean enough. Therefore, one only has to establish the MESERAN total counts that correspond to that level of contamination and relate the tests as being in compliance or not. The KCP has used this technique for years to control cleanliness and compare the abilities of different cleaners and cleaning processes to remove various 


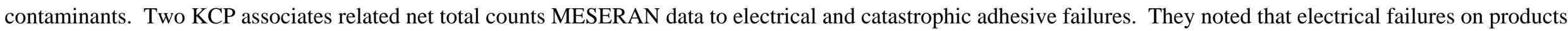

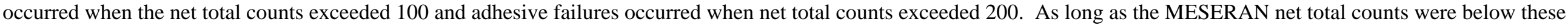
levels, no cleaning-related failures occurred, [4-9].

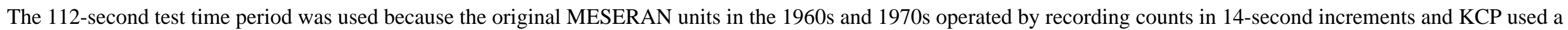

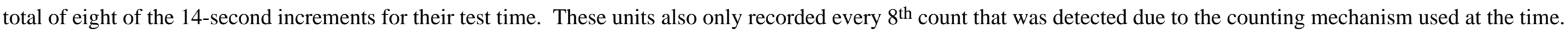

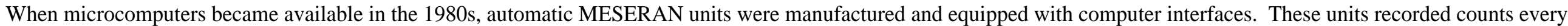

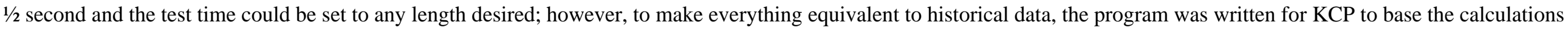
on 112 seconds with only every $8^{\text {th }}$ count reported.

\section{Newly Developed Method (Slope of Radiochemical Evaporation)}

A new Slope Method of analyzing the MESERAN data has been developed and improved during this project. The Slope Method is the slope of the evaporation of radiochemical (the post-peak portion of the curve shown in Figure 3 as the B-C line). Even though this method of analysis was known as a viable method since the 1960s and 1970s, it was not a practical method to use until microcomputers became widely available so the data could be manipulated easily. Some initial slope calculations were available with the automatic MESERAN units in the mid-to-late 1980s and early 1990s; however, KCP found them of limited use at the time. This project further developed the slope technology to its current state.

Based upon raw data minus background, Figure 5 illustrates three typical experimentally derived curves of natural logarithm (Ln or ln) counts per second minus background vs. time in seconds. Figure 5 is similar to Figure 4 except raw data from actual tests are shown.

In Figure 5, the upper curve represents a high level of organic residue, the middle curve represents a medium level of organic residue, and the lower curve represents a low level of organic residue. The scatter, particularly at the lower values, is due to the inherent randomness of radiation (the Poisson distribution in which the square root of each count total is the best estimate of one standard deviation). In an effort to reduce the effect of the randomness of radiation, the data (ln (counts - background) are "smoothed" from six seconds through 115 seconds (for 120-second length tests) and re-plotted. The smoothing is accomplished by 


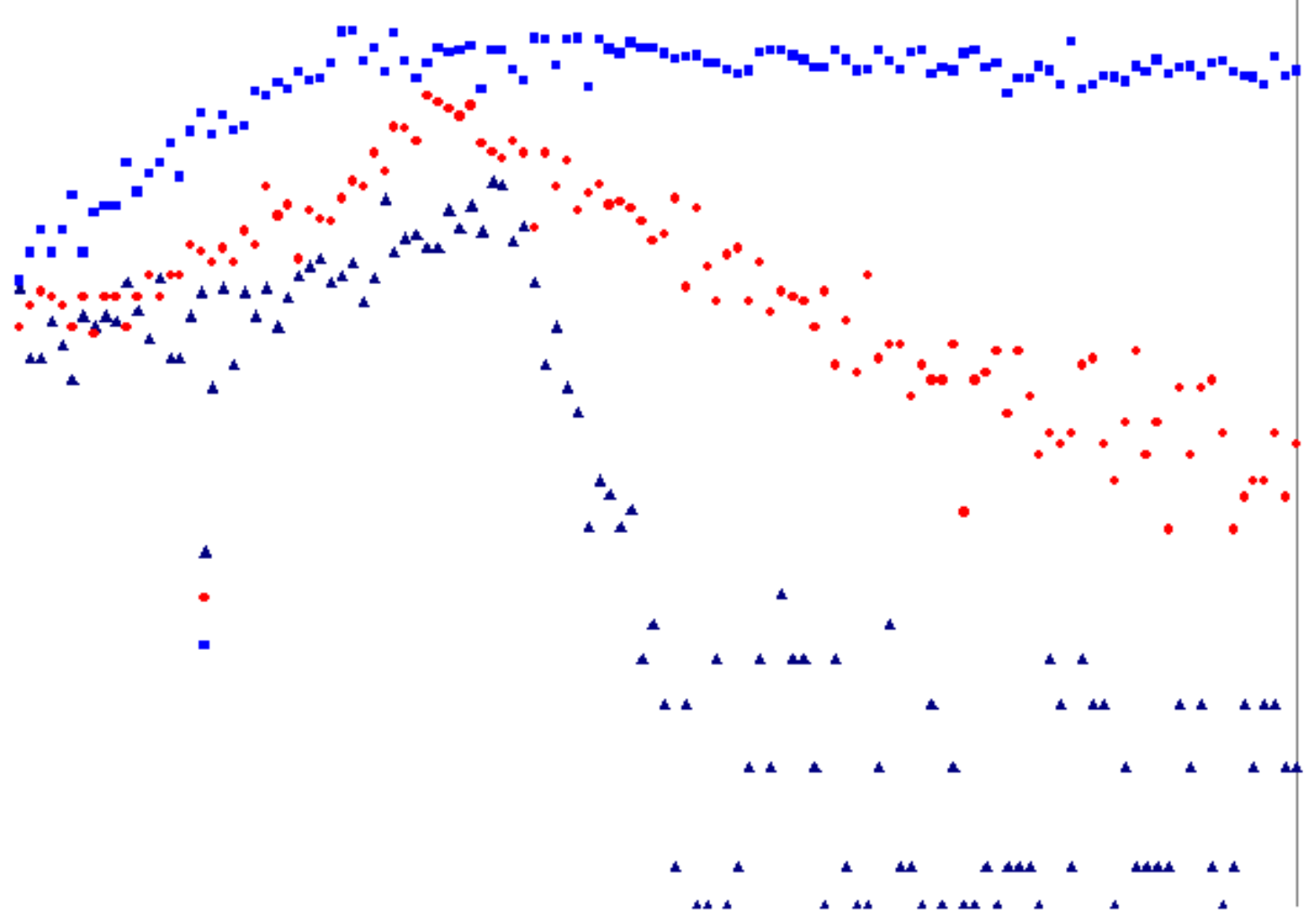

\section{Figure 5. Plot of Raw Data Showing Low, Medium, and High Levels of Organic Contamination}

summing the ln (counts - background) at the sixth second with the previous five seconds and the subsequent five seconds and dividing this number by 10 . A divisor of 10 is used instead of 11 because it is statistically sound to take the number of items being smoothed and subtract one from it because a degree of freedom is lost. This process is carried out through the $115^{\text {th }}$ second and the subsequent data are re-plotted as smoothed (ln (counts - background)) versus time. Figure 6 represents the same data as in Figure 5 except that the data in Figure 6 are logarithmically smoothed to increase the reliability of the individual points. The plotted smoothed curve is then analyzed via linear regression to determine the slope of the post-peak line (down to near background) which best fits the data representing the evaporative process. The determined slope is multiplied by $-10,000$ to convert it to a positive integer; this becomes the reported MESERAN slope value with units of smoothed (ln (counts - background))/sec $\mathrm{x}$ $(-10,0000)$. 


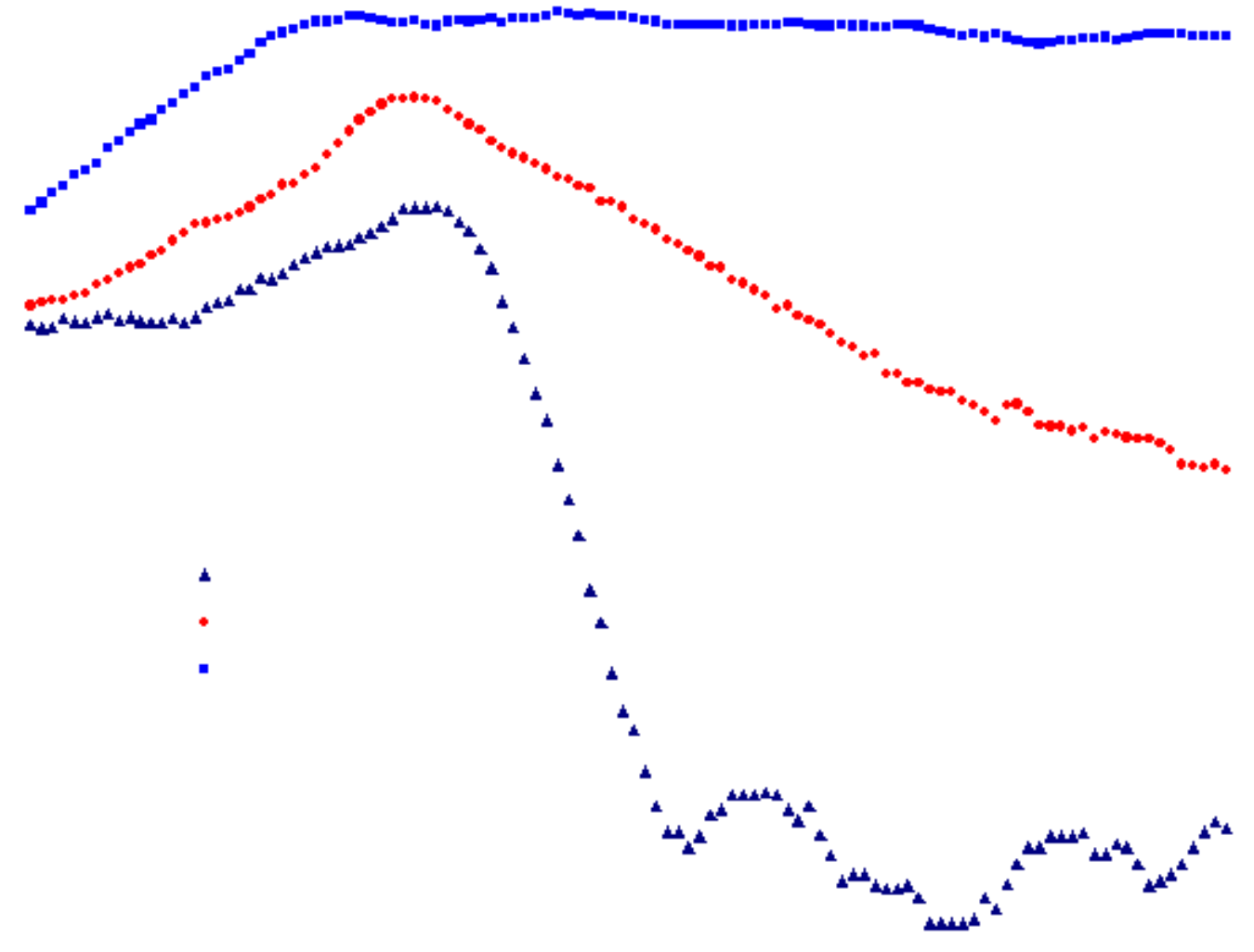

Medium, and High Levels of Organic

Figure 6. Logarithm Plot of Smoothed Data Showing Low, Contamination

\section{Comparison of Methods}

The Slope Method is more sensitive than the Total Counts Method, especially at low levels of contamination. For measuring microorganic residues, the Total Counts Method of analysis (i.e., the area under each overall curve based on actual counts) is valid from somewhat less than $200 \mathrm{ng}$ to approximately $100,000 \mathrm{ng}(100 \mu \mathrm{g}$ ). Higher total counts are indicative of higher organic residue levels and vice versa. The Slope Method of data analysis, normally based on statistically smoothed data and based on the log count vs. time relationship, increases the sensitivity of the lower limit markedly (to less than $1 \mathrm{ng}$ ) since the total counts method (the total area under each curve) approaches statistical insignificance somewhat below $200 \mathrm{ng}$. Expressed as a positive integer, the higher (or steeper) the slope, the cleaner the surface and conversely, the lower (or more flat) the slope, the higher the residue.

Both the Total Counts and Slope Methods of analysis can be used qualitatively or quantitatively. Qualitative measurements can be made by comparing the results obtained on the surface being tested to the results obtained from known clean standards for that particular surface. Quantitative measurements can be made by comparing the data to previously 


\section{Formulation of Calibration Solutions}

Calibrations of various contaminants (oils, greases, mold releases, rosin fluxes, etc) typically encountered in KCP production operations have been performed to develop calibration curves for these contaminants on substrates of interest. MESERAN total counts and slope results obtained can now be compared to the calibration curves to determine quantitative amounts of contamination detected, [11-14].

The volumetric dilution process for making calibration solutions is shown in Figure 7.

Volumetric dilutions were used to make contamination solutions for depositing known amounts of the organic contaminant on reference substrates (e.g., aluminum panels, stainless steel disks, glass cones, etc.). These calibrations were performed in the following manner. A master calibration solution was prepared in a 10-mL volumetric flask by dissolving $100 \mathrm{mg}$ of the organic contaminant in $10 \mathrm{~mL}$ of solvent (e.g., cyclopentane, methylene chloride, or hexane that has been double-distilled in an all-glass still with no grease in the joints - NVOR of these solvents are approximately $10 \mathrm{ppb}$ ). The master calibration solution was thoroughly mixed and

$1 \mathrm{~mL}$ of this solution was placed in another $10-\mathrm{mL}$ volumetric flask. The second volumetric flask was then diluted with the double-distilled solvent until the solution level was at $10 \mathrm{~mL}$ and this solution was thoroughly mixed. Subsequent dilutions were carried out in a similar fashion.

Ten microliters $(\mu \mathrm{L})$ of each calibration solution were deposited on the precleaned substrates and allowed to evaporate. This resulted in the following amounts of contamination on the substrates: $1 \mathrm{ng}, 10 \mathrm{ng}, 100 \mathrm{ng}, 1 \mu \mathrm{g}, 10 \mu \mathrm{g}$, and $100 \mu \mathrm{g}$. In some cases, intermediate levels were obtained by depositing $3 \mu \mathrm{L}$ and $5 \mu \mathrm{L}$ of the calibration solutions.

\section{Contaminants}

The contaminants chosen for calibrations are typically encountered in KCP production operations, and most have been evaluated for years when cleaning evaluations were performed and MESERAN Analyzers were used to determine how well the contaminants were removed. The following four rosin fluxes were evaluated: Kester 135, Kester 185, Kester 197, and Kester 1544. The following general contaminants were evaluated: WD-40 Oil, Apiezon Grease M, Starrett M-1 Lubricant, Vaseline, Dioctyl Phthalate (DOP), Carnauba Wax, OSR Mold Release, Thalco 550 Mold Release, RAM 225 Mold Release, Krylon Clear Covercoat Spray, EPON 828 Resin, Versamid 140 Curing Agent, Velva Sheen Mop Oil, Regal R \& O 32 Oil, a Hydrocarbon Blend (70\% Pennex N47 and 30\% Hangsterfer's Hard Cut \#511), Mineral Oil, and Hexatriacontane.

\section{$\underline{\text { Substrates }}$}




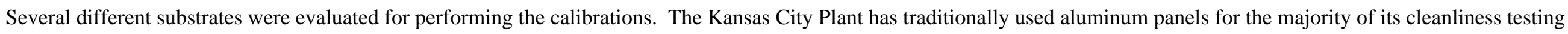

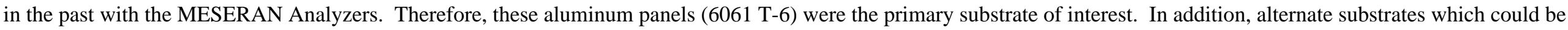

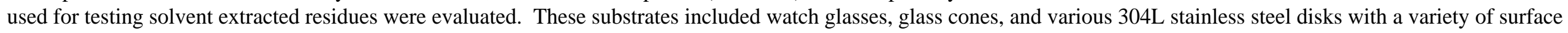
configurations (smooth, straight parallel grooves, spiral grooves, etc.).

\section{Cleaning Processes}

Several cleaning processes were evaluated to pre-clean the substrates to acceptable levels so the calibrations could be performed.

Cleaning processes evaluated for the aluminum panels included the following:

- Vapor degreasing and ultrasonic cleaning with trichloroethylene (TCE) using bench-top ultrasonic cleaner and small vapor degreaser followed by vacuum baking at $165^{\circ} \mathrm{F}$ for 1 hour,

- Spray cleaning, vapor degreasing, and ultrasonic cleaning with trichloroethylene (TCE) in F-1 Near Zero Emission Cleaner, and

- Spray cleaning, vapor degreasing, and ultrasonic cleaning with TCE in F-1 Near Zero Emission Cleaner followed by plasma cleaning in oxygen at 0.8 torr and 100 watts for 1 hour.

Cleaning processes evaluated for the watch glasses and glass cones included the following:

- Acid cleaning in sulfuric/nitric acid mixture $\left(\sim 1 \mathrm{~L}\right.$ of sulfuric acid and $\sim 5-10 \mathrm{~mL}$ of nitric acid) for 1 hour after mixture heated to $135^{\circ} \mathrm{C}$, rinse with tap water, rinse with distilled water, place in oven at $225^{\circ} \mathrm{F}$ for 4 hours,

- Acid cleaning as described above followed by plasma cleaning in oxygen at 0.8 torr and 100 watts for 1 hour, and

- Aqueous ultrasonic cleaning with a variety of aqueous cleaners (Brulin 815 GD and Dirl Lum 603 were the best) followed by a distilled water rinse and heating in a hot tube furnace.

Cleaning processes evaluated for the stainless steel disks included the following:

- Aqueous ultrasonic cleaning with either Brulin 815 GD or Dirl Lum 603 followed by a distilled water rinse and heating in a hot tube furnace at $390-425^{\circ} \mathrm{C}$ for $2-8$ hours,

- (1) Spray cleaning, vapor degreasing, and ultrasonic cleaning with TCE in F-1 Near Zero Emission Cleaner, (2) aqueous ultrasonic cleaning with Dirl Lum 603 (30 g per liter concentration) for 10 minutes at $140^{\circ} \mathrm{F}$, (3) rinsed in flowing deionized (DI) water for $15-30$ seconds, (4) DI water rinsed in ultrasonic cascade rinse station with 3 tanks ( 30 seconds in each tank) at $110-115^{\circ} \mathrm{F}$, (5) blown dry with filtered nitrogen, (6) baked for 30 minutes minimum at $220^{\circ} \mathrm{F}$ in a HEPA filtered convection oven with nitrogen flowing into the oven.

\section{Calibration Results}

Numerous evaluations were performed to identify "clean" substrates. Calibrations at low levels of contamination are impossible unless acceptably clean substrates can be reproduced. This project showed that there is more variability in high precision cleaning processes than expected. The MESERAN technique for analyzing cleanliness using the newly developed slope method of analysis was able to discern differences in cleaning processes at low levels of contamination previously not known to exist. Replicate samples cleaned in the exact same processes at the same times are not necessarily cleaned to the same levels of cleanliness. In fact, multiple tests in different areas on the same panel show varying levels of cleanliness (in the submonolayer region - below about $200 \mathrm{ng}$ ). Therefore, performing calibrations at levels below this amount requires significant care in how the samples are handled and packaged. In addition, in many cases numerous samples had to be cleaned to find enough acceptable samples to perform the calibrations at the low nanograms levels.

Plasma cleaning yields cleaner surfaces than wet chemical cleaning alone. Previously, it was thought that plasma-cleaned surfaces could not be tested with the MESERAN Analyzer because the activity generated on the surfaces by plasma cleaning would interfere with the evaporation of the radiochemical. Tests performed previously on parts immediately after plasma cleaning showed an increase in total counts because the radiochemical was retained by the active surface. Evaluations were conducted on this project 


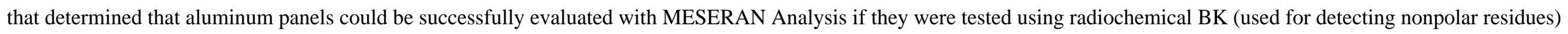

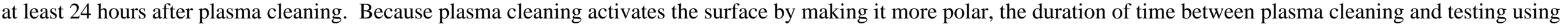

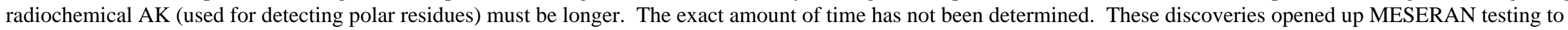
plasma cleaned parts and proved that plasma cleaning does improve the cleanliness of parts.

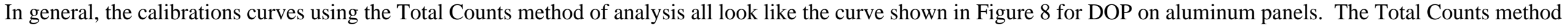
loses sensitivity below a few hundred nanograms.

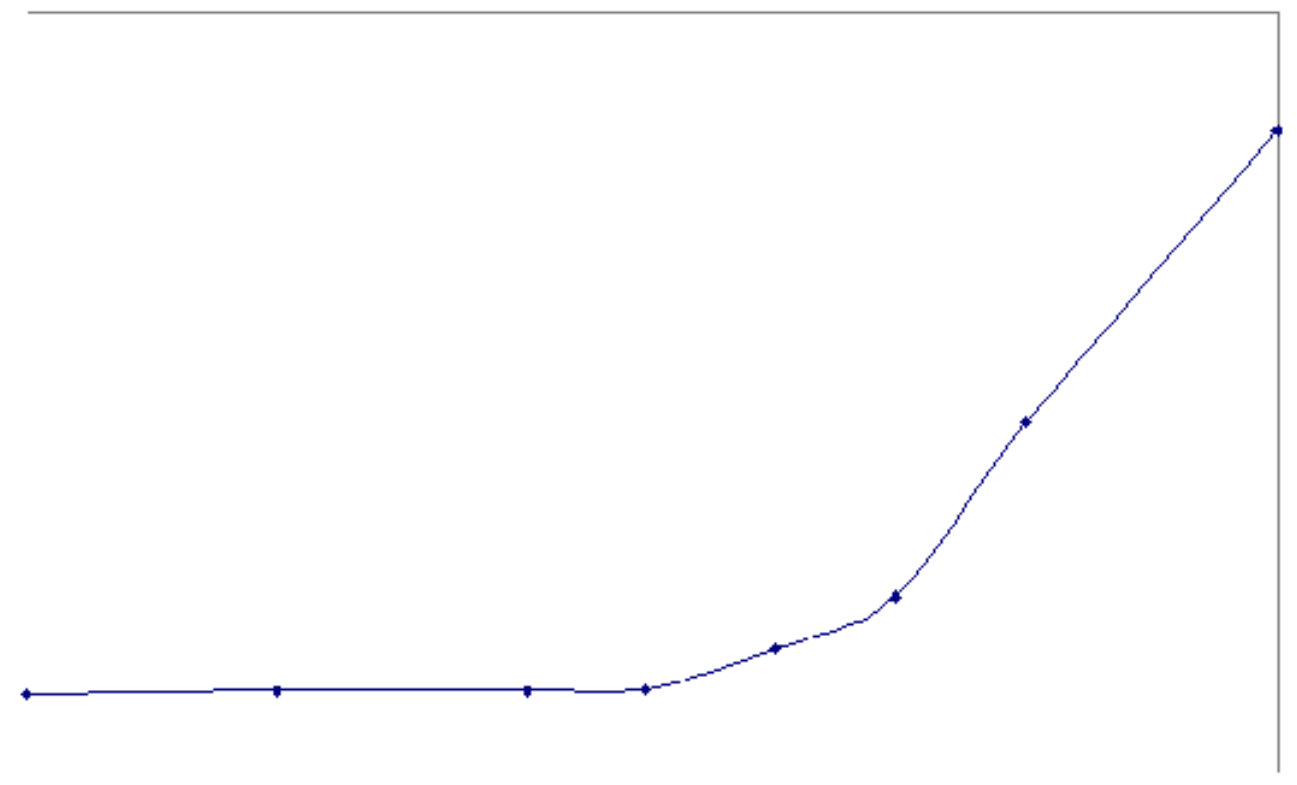

Figure 8. Total Counts Calibration Curve for DOP on Aluminum Panels

The calibration curves using the Slope method of analysis all generally look like the curve shown in Figure 9 for DOP on aluminum panels. The Slope method shows increased sensitivity in the low nanogram region. 


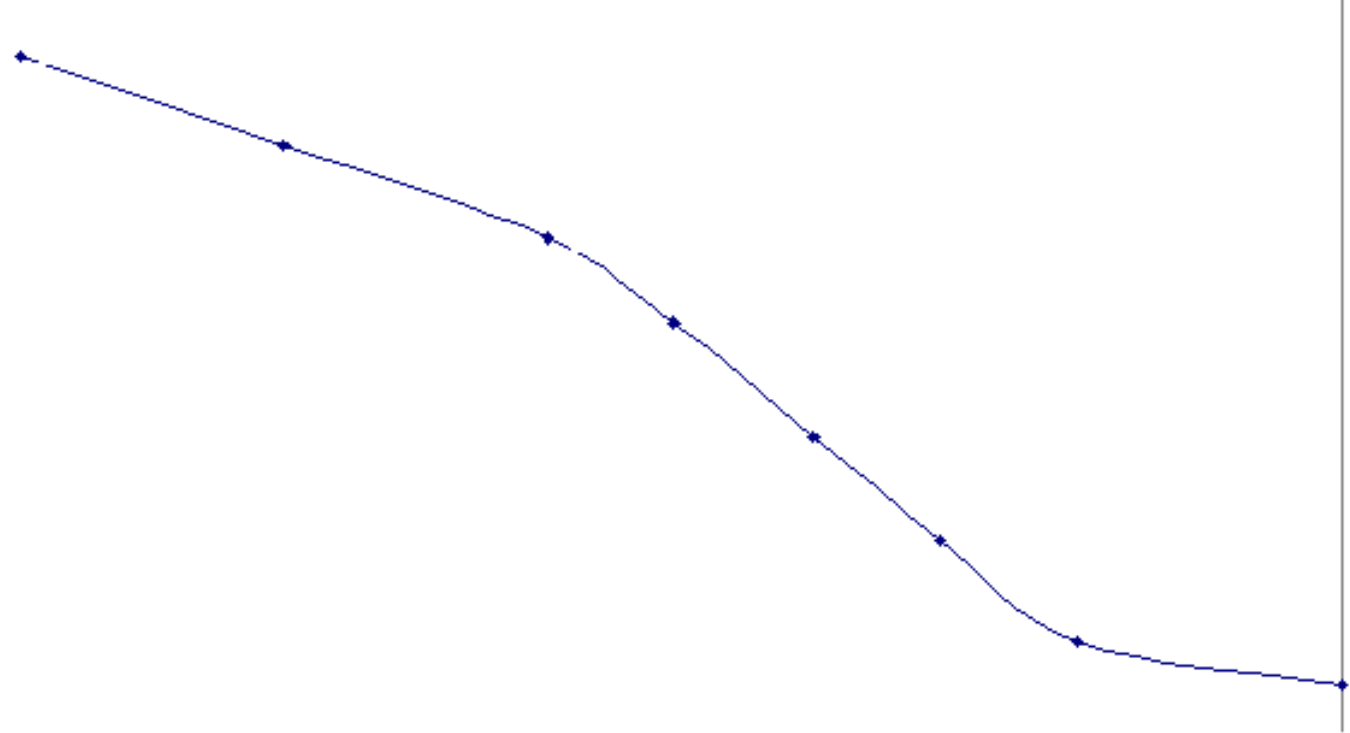

Figure 9. Low Variance Slope Calibration Curve for DOP on Aluminum Panels

Calibrations were performed on the contaminants, and the results were analyzed using the Total Counts method and several Slope methods. The primary Slope method is the Low Variance Slope described previously in the Newly Developed Method (Slope of Radiochemical Evaporation) section of the report. In addition, slopes were analyzed at 20-, 30-, and 50-point lengths to determine if any of those slopes gave additional definition. Longer slope lengths (30 and 50 points) are only applicable for higher amounts of contamination. They may provide somewhat better definition at the high microgram levels, but are generally useless at lower levels. The 20-point length slopes reduce some of the variability seen in the low variance slopes and may prove to be acceptable for levels down to about $10 \mathrm{ng}$. Overall the low variance slopes and 20-point length slopes are the most valuable.

Various different stainless steel disks were evaluated. Therefore, not all of the calibrations performed on stainless steel were performed on the same type of disks (different machine grooves). Calibrations are definitely different depending upon the substrate used.

Surface roughness was found to be a greater than expected factor in MESERAN testing. Consistent surfaces are needed to perform accurate calibrations. In addition, calibrations for a particular contaminant do vary on different substrates. Anyone wanting quantifiable contamination data on a particular substrate using direct testing will need to have calibrations performed on that substrate to ensure differences in surface roughness can be eliminated.

Stainless steel disks have been designed with specified machine grooves that allow consistent MESERAN results to be obtained. These disks can be used as reference surfaces for evaluating cleaning processes and cleanliness levels of various products. Parts being analyzed can be extracted with a solvent to remove any potential organic contamination, and the solvent extract can then be evaporated using the MicroSolventEvaporator (developed on a Cooperative Research and Development Agreement - CRADA) to deposit the 
The orientation and depth of the grooves affected the MESERAN results. Straight, parallel grooves allow the contaminants (especially ones with low surface tension) to run or expand in the direction of the grooves. The longer the contamination is left there prior to testing, the more it will expand (especially at higher contamination levels). The radiochemical solution will also run in the direction of the grooves. Circular or spiral grooves increase the reproducibility of the data. They act as a self-centering device for the deposition of the contaminants as well as for the deposition of the radiochemical solution during MESERAN testing. Shallow grooves (0.002 inch or less) appear to lead to increased sensitivity at lower contamination levels (less than $100 \mathrm{ng}$ ). Deeper grooves (i.e., $0.005 \mathrm{inch}$ ) lead to decreased sensitivity at lower contamination levels (less than 100 $\mathrm{ng}$ ) and increased sensitivity at higher contamination levels (greater than $1 \mu \mathrm{g}$ ).

Many contaminants have very similar calibration curves for the same contamination levels. Hydrocarbons tend to test very similarly. However, there are differences that exist in the responses for the wide variety of contaminants evaluated on this project. It may be possible to develop a calibration curve for a general hydrocarbon contaminant for a particular surface; however, it will be much more difficult to develop a general calibration curve for all contaminants.

During the course of the project, problems were encountered with the radiochemical solutions. Some radiochemical solutions appeared to be contaminated and therefore did not test well on clean substrates or low levels of contamination. The new Slope method of analysis was able to detect this problem even though the Total Count method did not. KCP utilized the Six Sigma

Process and performed tests that showed that some of the radiochemical solutions The MESERAN Company received from their supplier were contaminated. In addition, The MESERAN Company depleted their supply of radiochemicals and had to reorder new tetrabromoethane-C14 (radiochemical A) and tridecane-C14 (radiochemical B). The newly synthesized tridecane-C14/cyclopentane (BK) did not test the same as the BK used in previously performed calibrations. The tetrabromoethane-C14 also appears to potentially have shelf life issues. Some of the bromine may evolve over time in the concentrated ampules The MESERAN Company receives from its supplier. This results in a mixture of materials instead of just pure tetrabromoethane-C14. Again, the Slope method of analysis detects this whereas the Total Count method does not. Great care must be taken to ensure that each lot of radiochemical solution is identical for the Slope method to be used without performing calibrations for each new lot of radiochemical.

The MESERAN program has been updated to calculate the various slope methods developed on this project. In addition, a program was developed to convert MESERAN data taken on the KCP's automatic MESERAN Analyzers (i.e., various cleaning related evaluations since 1985) into data that can be analyzed by the various slope methods.

The calibration results are shown in the following Appendices:

Appendix A - Calibration Curves for Kester 135 Rosin Flux on Aluminum Panels and Stainless Steel Disks Appendix B - Calibration Curves for Kester 185 Rosin Flux on Aluminum Panels and Stainless Steel Disks Appendix C - Calibration Curves for Kester 197 Rosin Flux on Aluminum Panels and Stainless Steel Disks Appendix D - Calibration Curves for Kester 1544 Rosin Flux on Aluminum Panels and Stainless Steel Disks Appendix E - Calibration Curves for WD-40 Oil on Aluminum Panels and Stainless Steel Disks

Appendix F - Calibration Curves for Apiezon Grease M on Aluminum Panels and Stainless Steel Disks Appendix G - Calibration Curves for Starrett M-1 Lubricant on Aluminum Panels and Stainless Steel Disks Appendix H - Calibration Curves for Vaseline on Aluminum Panels and Stainless Steel Disks Appendix I - Calibration Curves for Dioctyl Phthalate (DOP) on Aluminum Panels and Stainless Steel Disks Appendix J - Calibration Curves for Carnauba Wax on Aluminum Panels and Stainless Steel Disks Appendix K - Calibration Curves for OSR Mold Release on Aluminum Panels and Stainless Steel Disks Appendix L - Calibration Curves for Thalco 550 Mold Release on Aluminum Panels and Stainless Steel Disks

Appendix M - Calibration Curves for RAM 225 Mold Release on Aluminum Panels and Stainless Steel Disks Appendix N - Calibration Curves for Krylon Clear Covercoat Spray on Aluminum Panels and Stainless Steel Disks Appendix O - Calibration Curves for EPON 828 Resin on Aluminum Panels and Stainless Steel Disks 
Appendix U - Calibration Curves for Hexatriacontane on Stainless Steel Disks

\section{Accomplishments}

Calibrations of various organic contaminants typically encountered in Kansas City Plant operations were developed for aluminum and stainless steel substrates.

A new analysis method was developed to enhance the ability of MESERAN Analyzers to detect organic contamination and yield quantitative data in the microgram and nanogram levels. The MESERAN program has been updated to calculate the various slope methods developed on this project. In addition, a program was developed to convert MESERAN data taken on KCP's automatic MESERAN Analyzers (i.e., various cleaning related evaluations since 1985) into data that can be analyzed by the various slope methods.

Numerous evaluations were performed to identify "clean" substrates. It was shown that there is more variability in high precision cleaning processes than expected. The MESERAN technique for analyzing cleanliness using the newly developed slope method of analysis was able to discern differences in cleaning processes at low levels of contamination previously not known to exist. Replicate samples cleaned in the exact same processes at the same times are not necessarily cleaned to the same levels of cleanliness. In fact, multiple tests in different areas on the same substrate show varying levels of cleanliness (in the submonolayer region - below about 200 ng).

Plasma cleaning yields cleaner surfaces than wet chemical cleaning alone. Previously, it was thought that plasma cleaned surfaces could not be tested with the MESERAN Analyzer because the activity generated on the surfaces by plasma cleaning would interfere with the evaporation of the radiochemical. Tests performed previously on parts immediately after plasma cleaning showed an increase in total counts because the radiochemical was retained by the active surface. Evaluations were conducted on this project that determined that aluminum panels could be successfully evaluated with MESERAN Analysis if they were tested using radiochemical BK (used for detecting nonpolar residues) at least 24 hours after plasma cleaning. These discoveries opened up MESERAN testing to plasma-cleaned parts and proved that plasma cleaning does improve the cleanliness of parts.

Surface roughness was found to be a greater-than-expected factor in MESERAN testing. Consistent surfaces are needed to perform accurate calibrations. In addition, calibrations for a particular contaminant do vary on different substrates. Anyone wanting quantifiable contamination data on a particular substrate using direct testing will need to have calibrations performed on that substrate to ensure differences in surface roughness can be eliminated.

Stainless steel disks have been designed with specified machine grooves that allow consistent MESERAN results to be obtained. Circular or spiral grooves increase the reproducibility of the data. They act as a self-centering device for the deposition of the contaminants as well as for the deposition of the radiochemical solution during MESERAN testing. These disks can be used as references surfaces for evaluating cleaning processes and cleanliness levels of various products. Parts being analyzed can be extracted with a solvent to remove any potential organic contamination and the solvent extract can then be evaporated using the MicroSolventEvaporator (developed on a Cooperative Research and Development Agreement - CRADA) to deposit the extracted residue on the disks. The disks can then be tested with the MESERAN Analyzer and the data compared to the calibrations performed on this project (or additional calibrations for specific contaminants of interest) so quantifiable contamination amounts can be determined.

During the course of the project, problems were encountered with the radiochemical solutions. Some radiochemical solutions appeared to be contaminated and therefore did not test well on clean substrates or low levels of contamination. The new Slope method of analysis was able to detect this problem even though the Total Count method did not. KCP utilized the Six Sigma Process and performed tests that showed that some of the radiochemical solutions The MESERAN Company received from their supplier were contaminated.

The technology developed on this project has already been used to support the KCP as follows: 
- Obtain new business for testing NVRs for Lawrence Livermore National Laboratory (LLNL) on the NIF project,

- Evaluate KCP's ability to clean PEPC LRU NIF hardware for LLNL in support of a \$1.2-million reimbursable order,

- Evaluate contamination levels on LAC Shells,

- Evaluate contamination for LED Bond Analysis project in Telemetry,

- Evaluate contamination levels on the Detector Hook Assembly,

- SMRI support for ultrasonic cleaning equipment,

- Evaluate cleaning ability of various atmospheric plasma equipment for Advanced Design and Production Technologies (ADAPT) projects.

Additional work currently scheduled that will utilize the technology developed on this project include:

- Cleaning evaluations performed for Sandia National Laboratory/New Mexico and Pantex to evaluate alternate wiping solvents,

- Evaluate the ability of plasma cleaning to remove organic contamination from pits for Los Alamos.

\section{References}

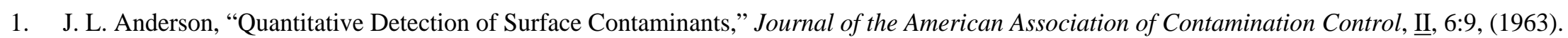

2. J. L. Anderson, et. al. "Measurement and Evaluation of Surfaces and Surface Phenomena by Evaporative Rate Analyses," Journal of Paint Technology, Vol. 40, No. 523, 320-327, (August, 1968).

3. J. L. Anderson, "Evaporative Rate Analysis: Its First Decade.” A chapter in Characterization of Metal and Polymer Surfaces. L. H. Lee, Editor, 2 , Academic Press, Inc., New York, 409-427, (1977). This paper summarizes all known references prior to 1975.

4. L. C. Jackson, Solubility Parameters and Evaporative Rate Analyses in Organic Residue Characterization (Topical Report). UNCLASSIFIED. Bendix Kansas City Division: BDX-613-1099, September 1974 (Available from NTIS).

5. L. C. Jackson, "How to Select a Substrate Cleaning Solvent," Adhesives Age, 22-31, (December 1974).

6. L. C. Jackson, "Solvent Cleaning Process Efficiency," Adhesives Age, 19, 31-34, (July 1976).

7. L. C. Jackson, "Removal of Silicone Grease and Oil Contaminants," Adhesives Age, 28 (4), 29-32, (1977).

8. L. C. Jackson, Contaminant Cleaning for Critical Electrical Assembly Areas, (Final Report). UNCLASSIFIED. Bendix Kansas City Division: BDX-613-1695, February 1978 (Available from NTIS).

9. M. G. Benkovich, "Solvent Substitution for Electronic Products," International Journal of Environmentally Conscious Manufacturing, 1, No. 1, 27-32 (1992).

10. M. G. Benkovich and J. L. Anderson, "Measurement of Organic Residues on Surfaces to a Low Fraction of a Monolayer," Precision Cleaning, 16-28, (May 1996). This paper includes many of the more current references to MESERAN technology.

11. M. G. Benkovich and J. L. Anderson, "Quantification of MicroOrganic Residues to Low Nanogram Levels," Precision Cleaning '96 Proceedings, 115-122, (1996).

12. J. L. Anderson, R. F. Russell, and M. G. Benkovich, "Quantitative Measurement of Extremely Low Levels of Non-Volatile Residues (NVR) on Surfaces and in Liquids," Precision Cleaning '97 Proceedings, 96-108, (1997).

13. J. L. Anderson, R. F. Russell, and M. G. Benkovich, "Solvent NVR: A Problem and a Solution," CleanTech '98 Proceedings, 331-340, (1998).

14. MESERAN Analyzer Literature, ERA Systems Inc., The MESERAN Company, Chattanooga, TN. 


\section{Appendix A}

Calibration Curves for Kester 135 Rosin Flux on Aluminum Panels and Stainless Steel Disks

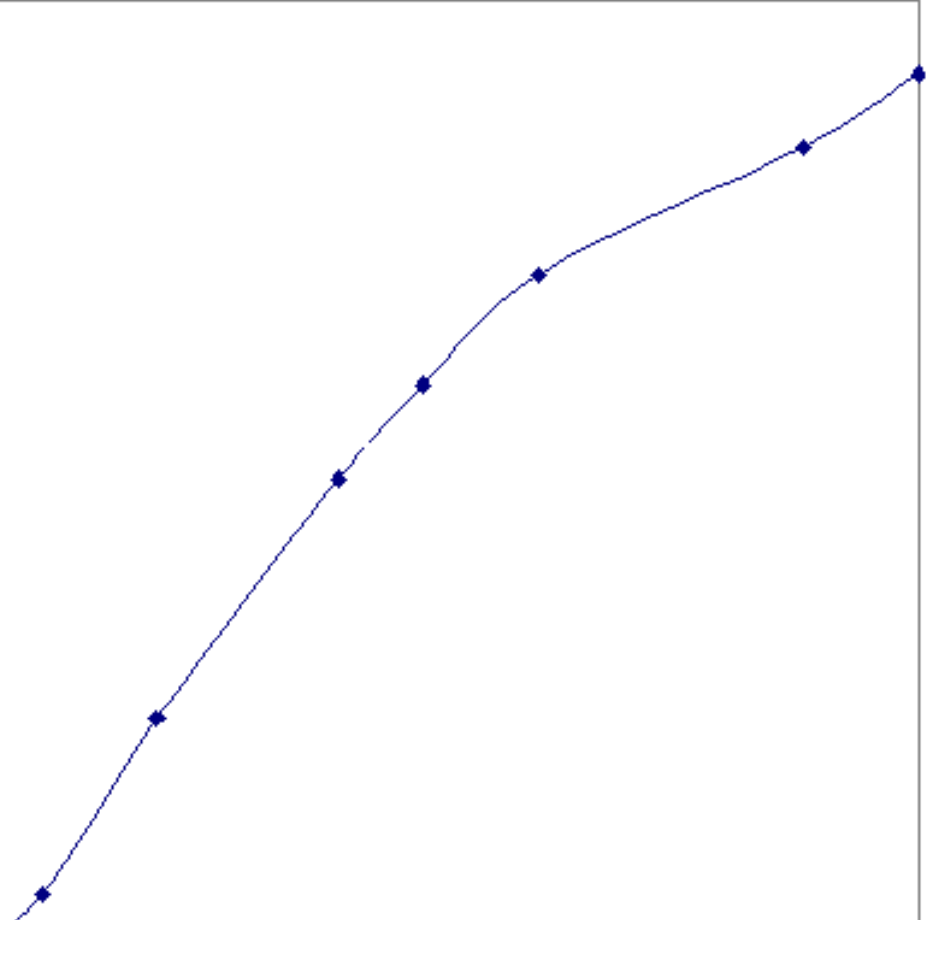


<smiles>CCCCCC</smiles> 

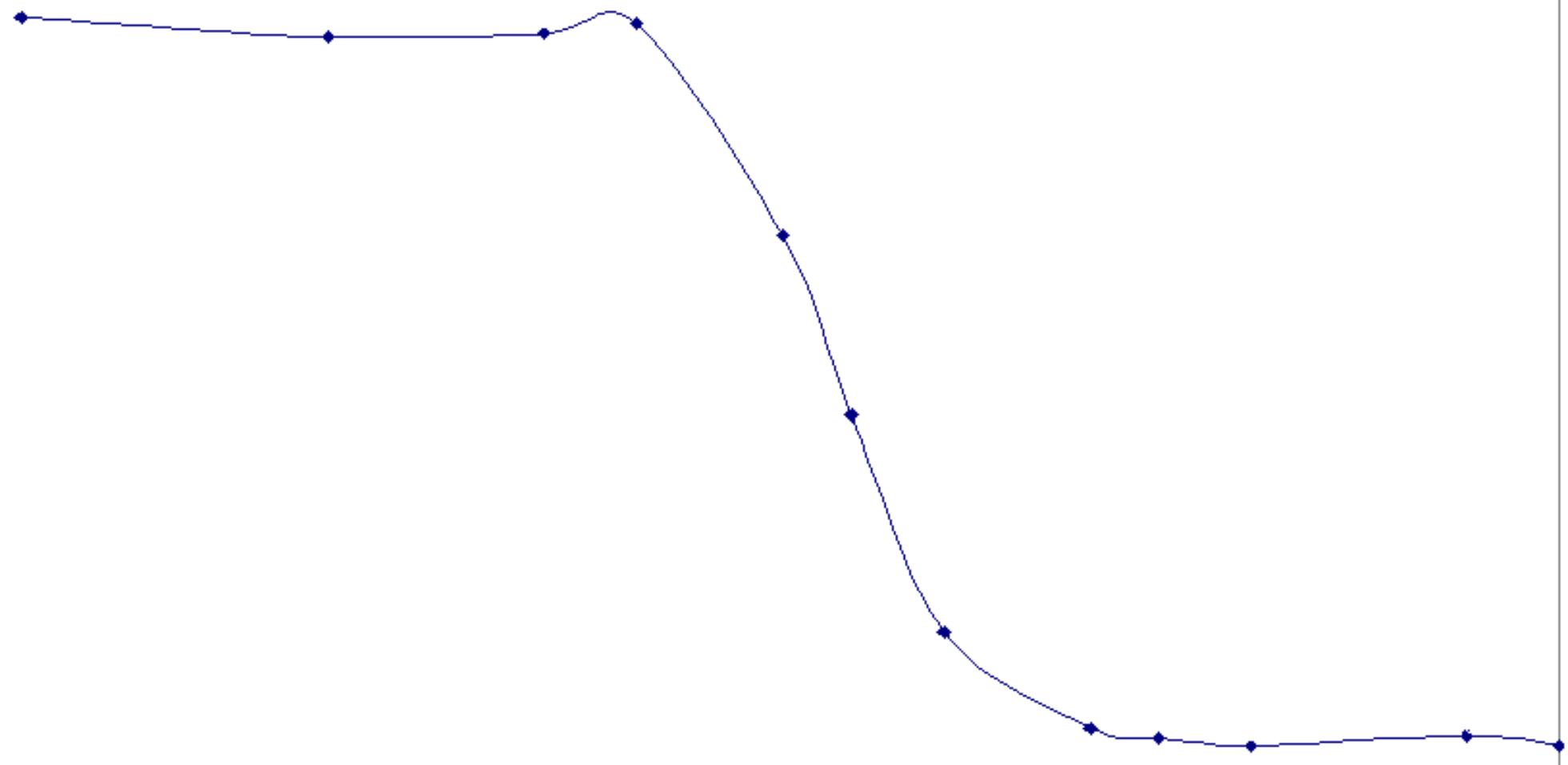
<smiles>CCCCCCCC</smiles> 
FY98 Annual Report

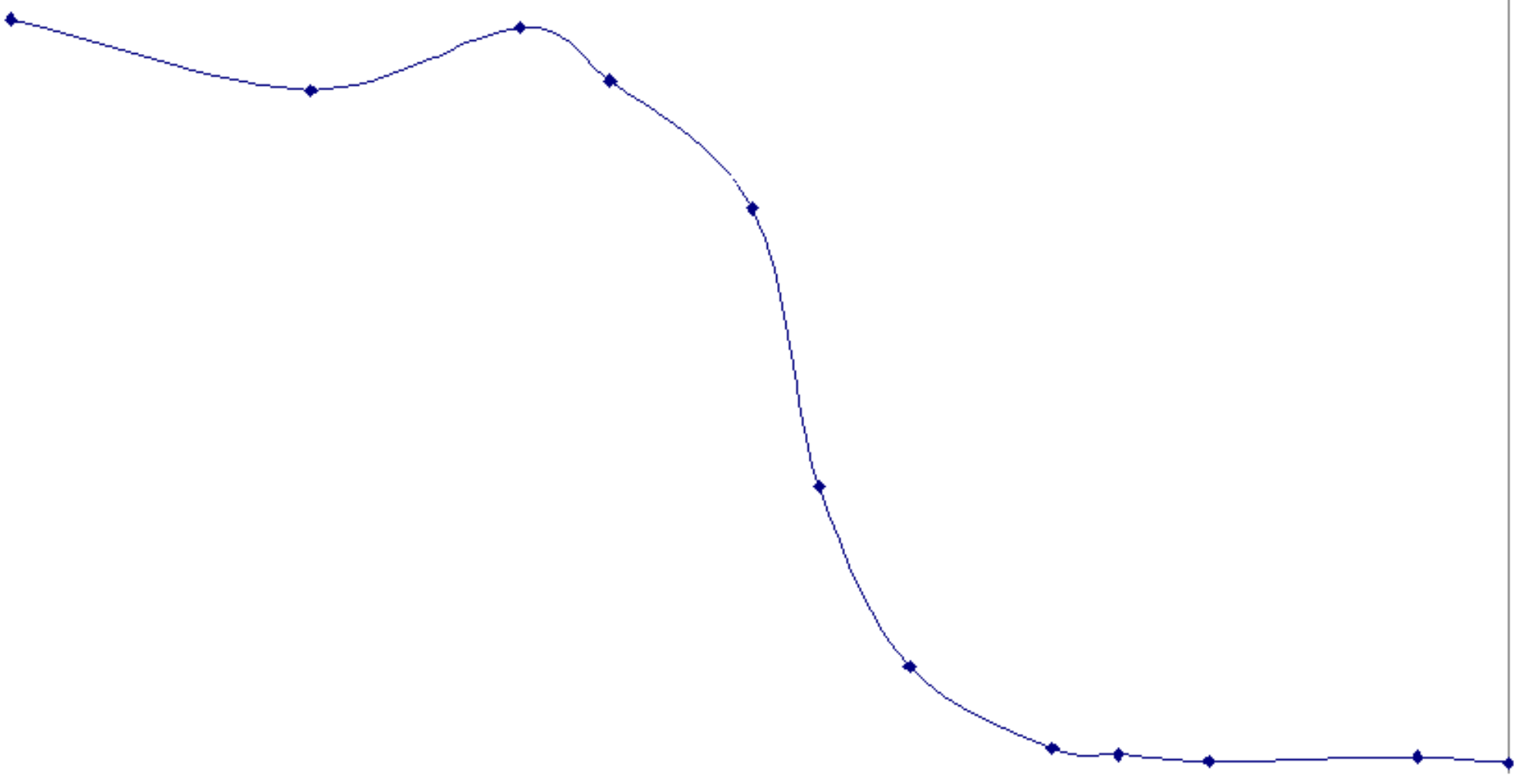

file://C|/ManualConversion/HTML/6792benk.htm (25 of 250)4/9/2004 4:05:24 AM 


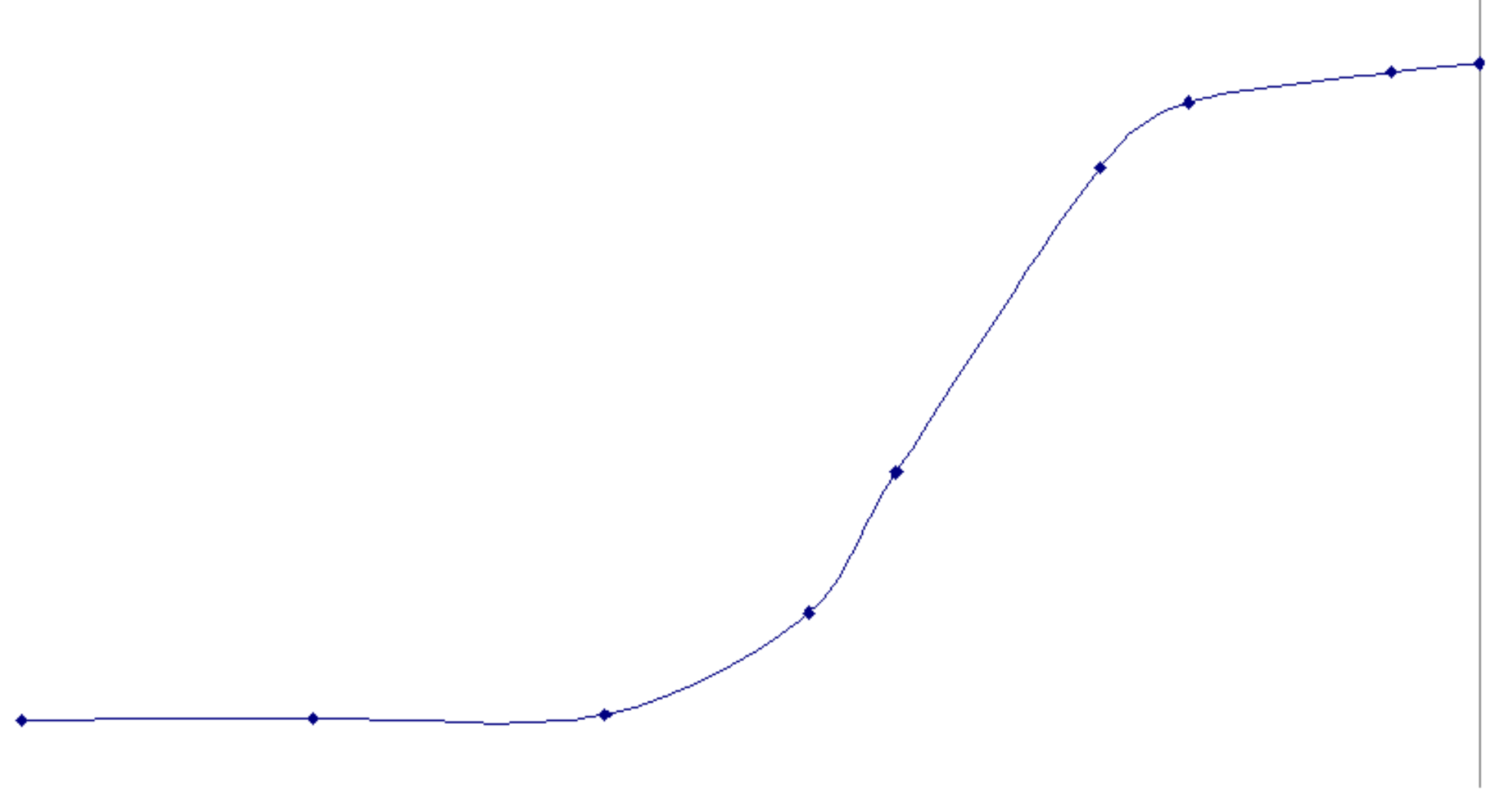


<smiles>C=CCCC</smiles> 
FY98 Annual Report

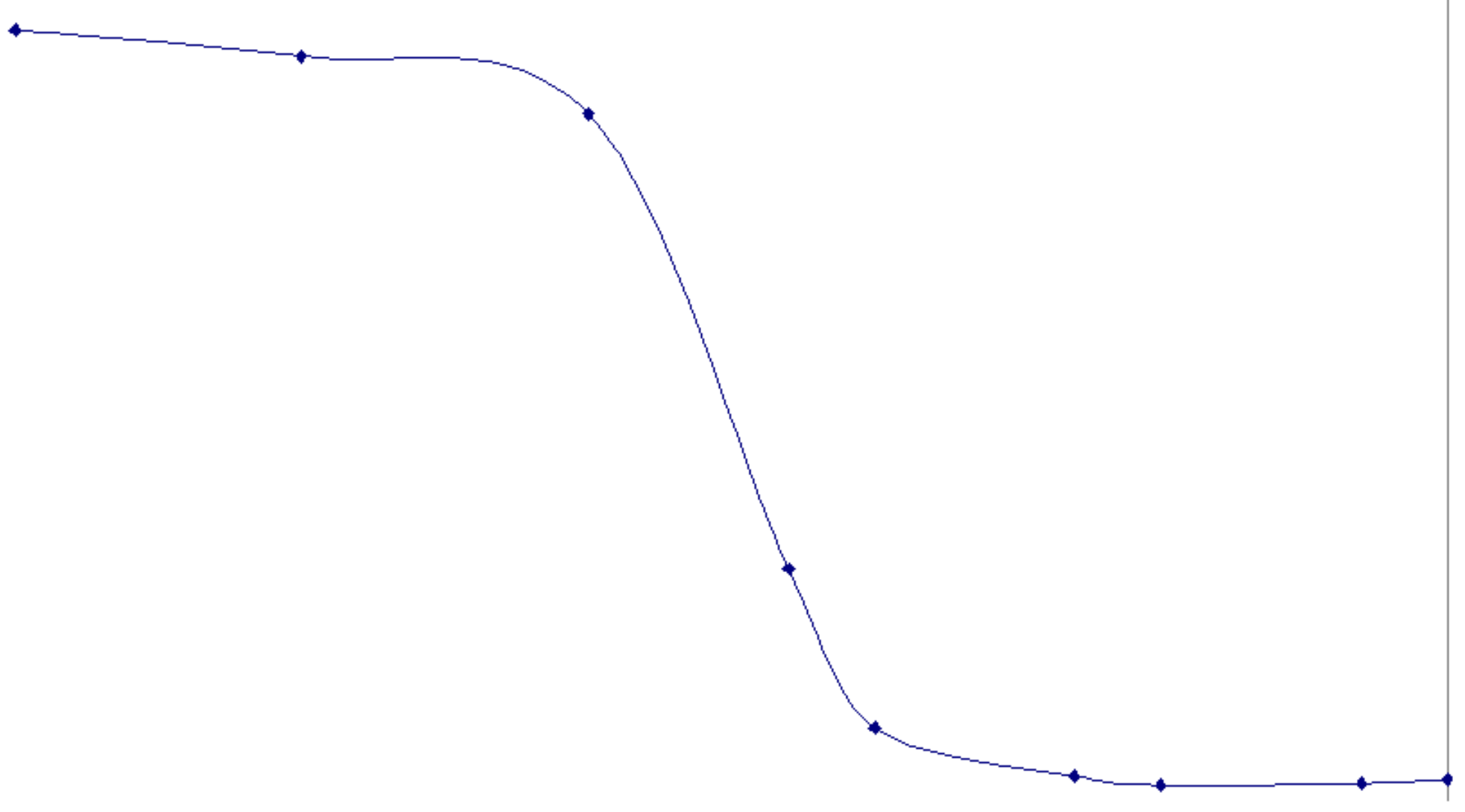

file:///C//ManualConversion/HTML/6792benk.htm (28 of 250)4/9/2004 4:05:24 AM 

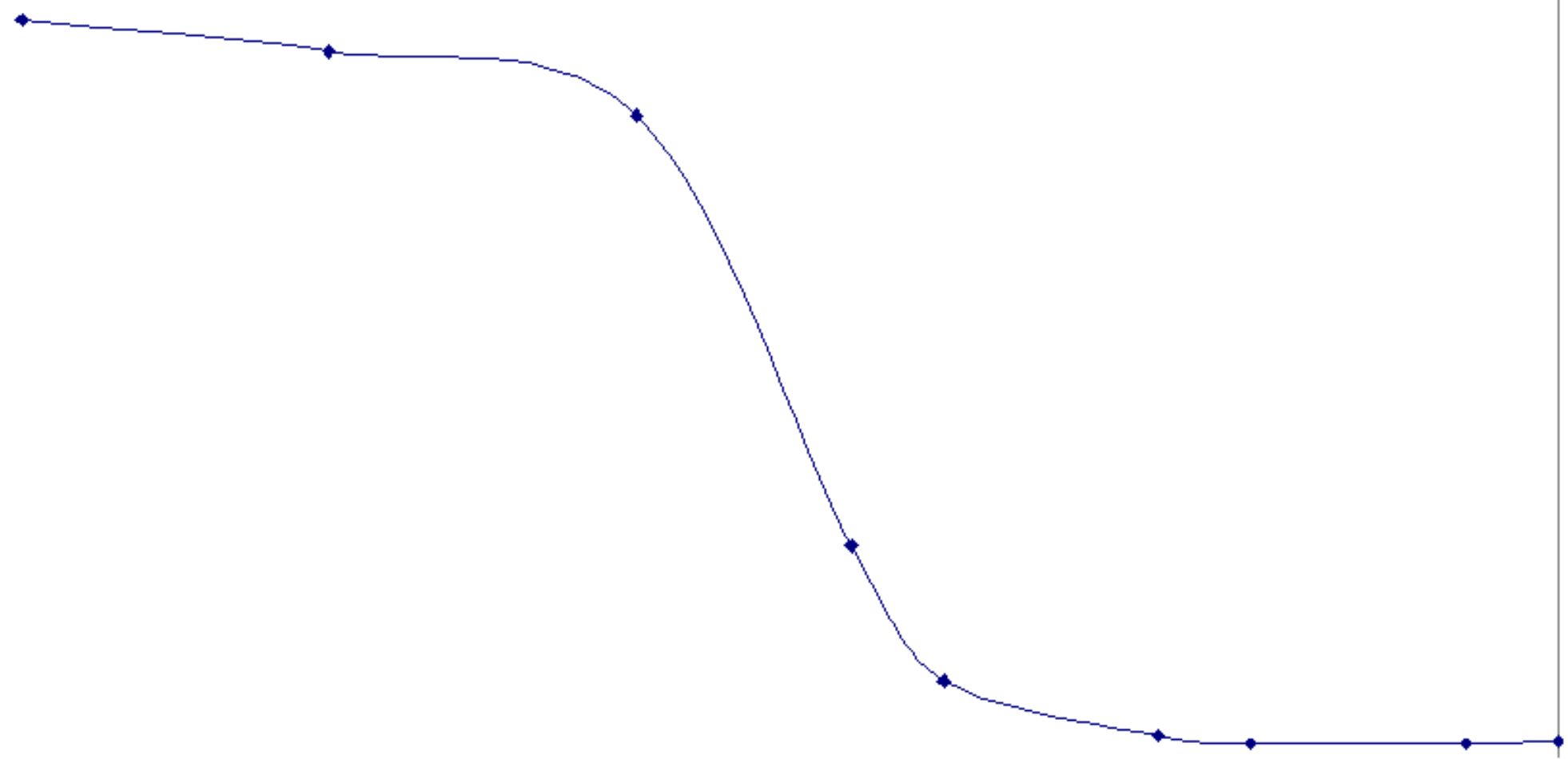

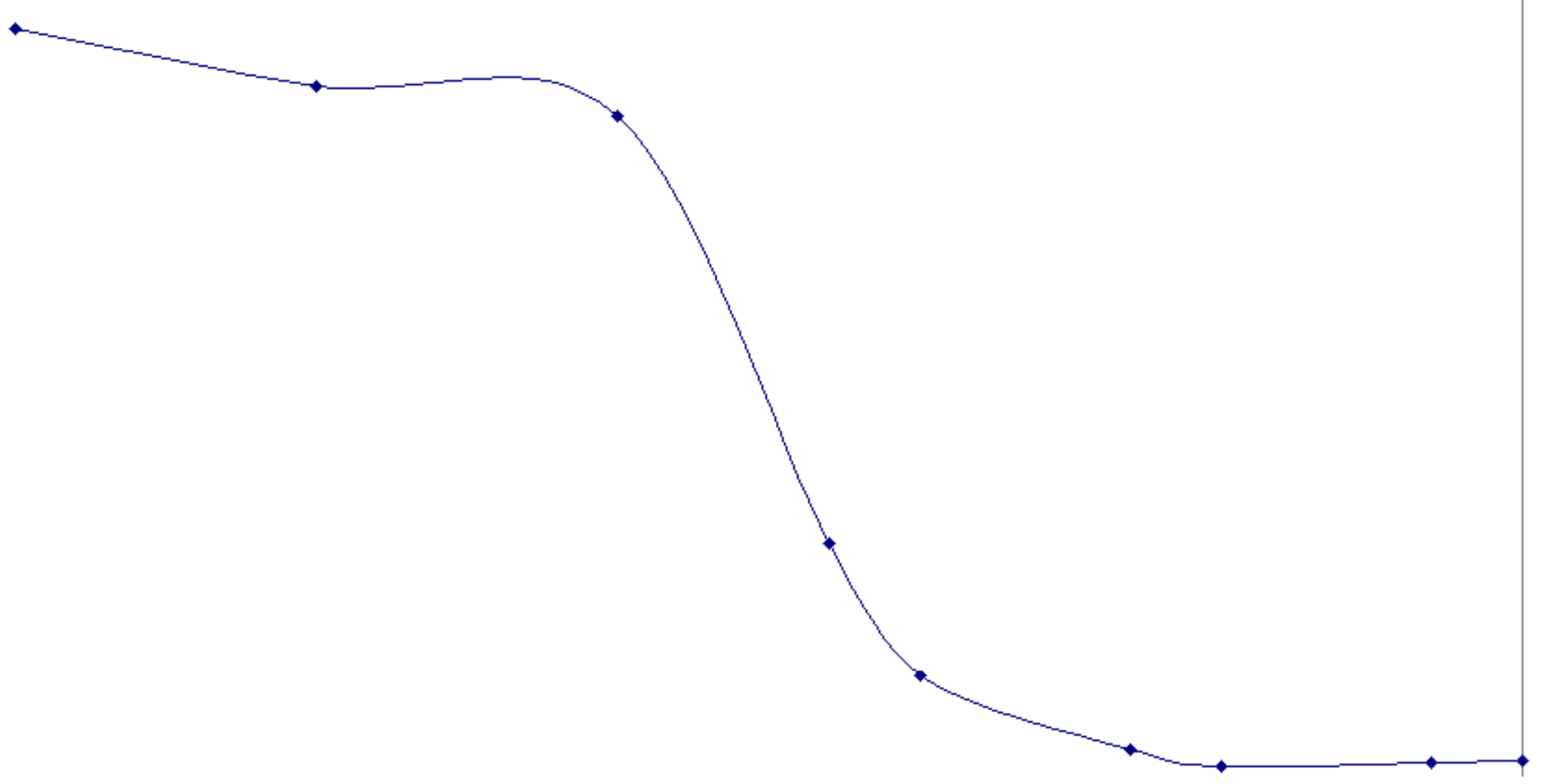
Appendix B

Calibration Curves for Kester 185 Rosin Flux

on Aluminum Panels and Stainless Steel Disks

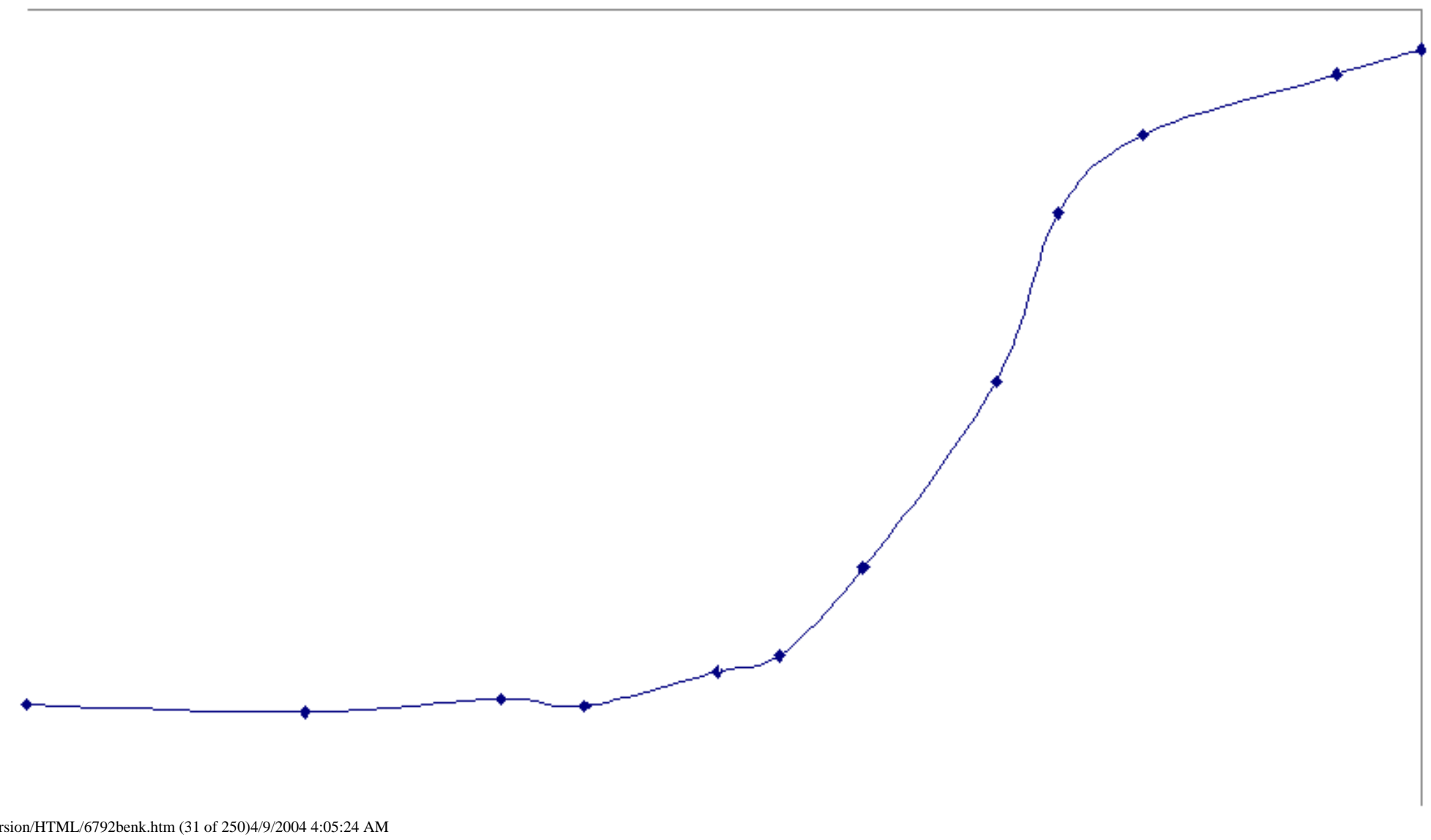


<smiles>CCCCCC</smiles> 


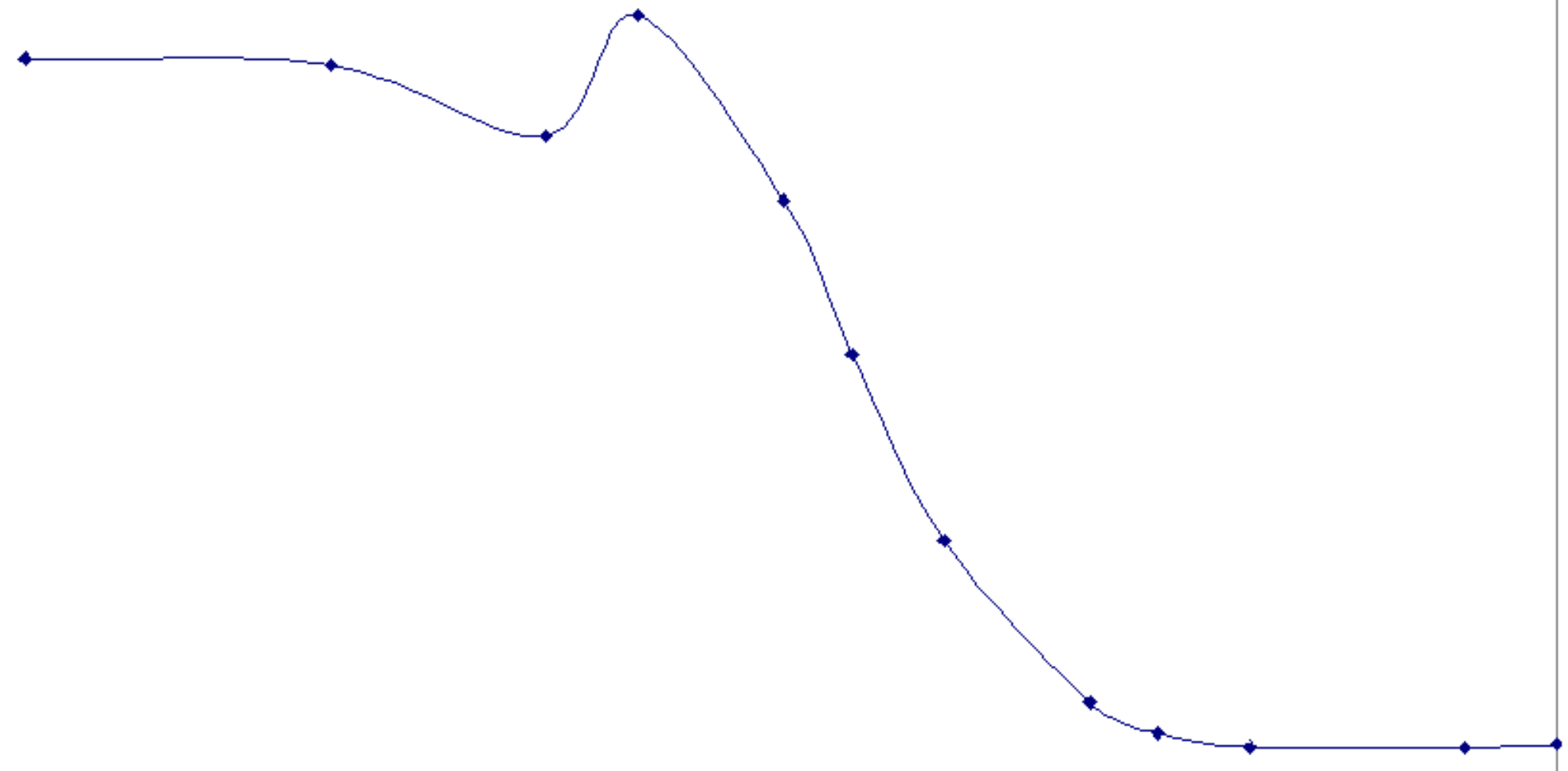




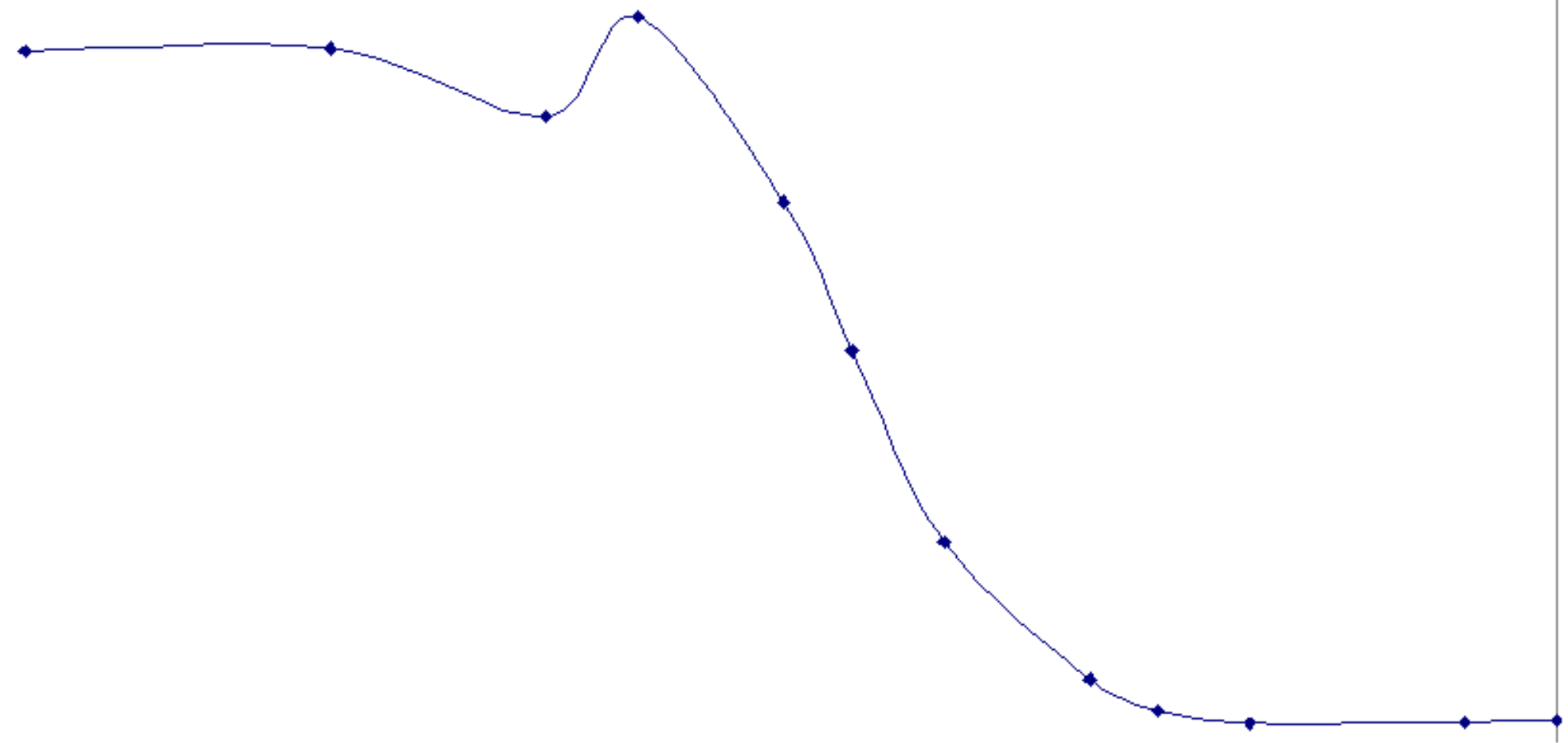




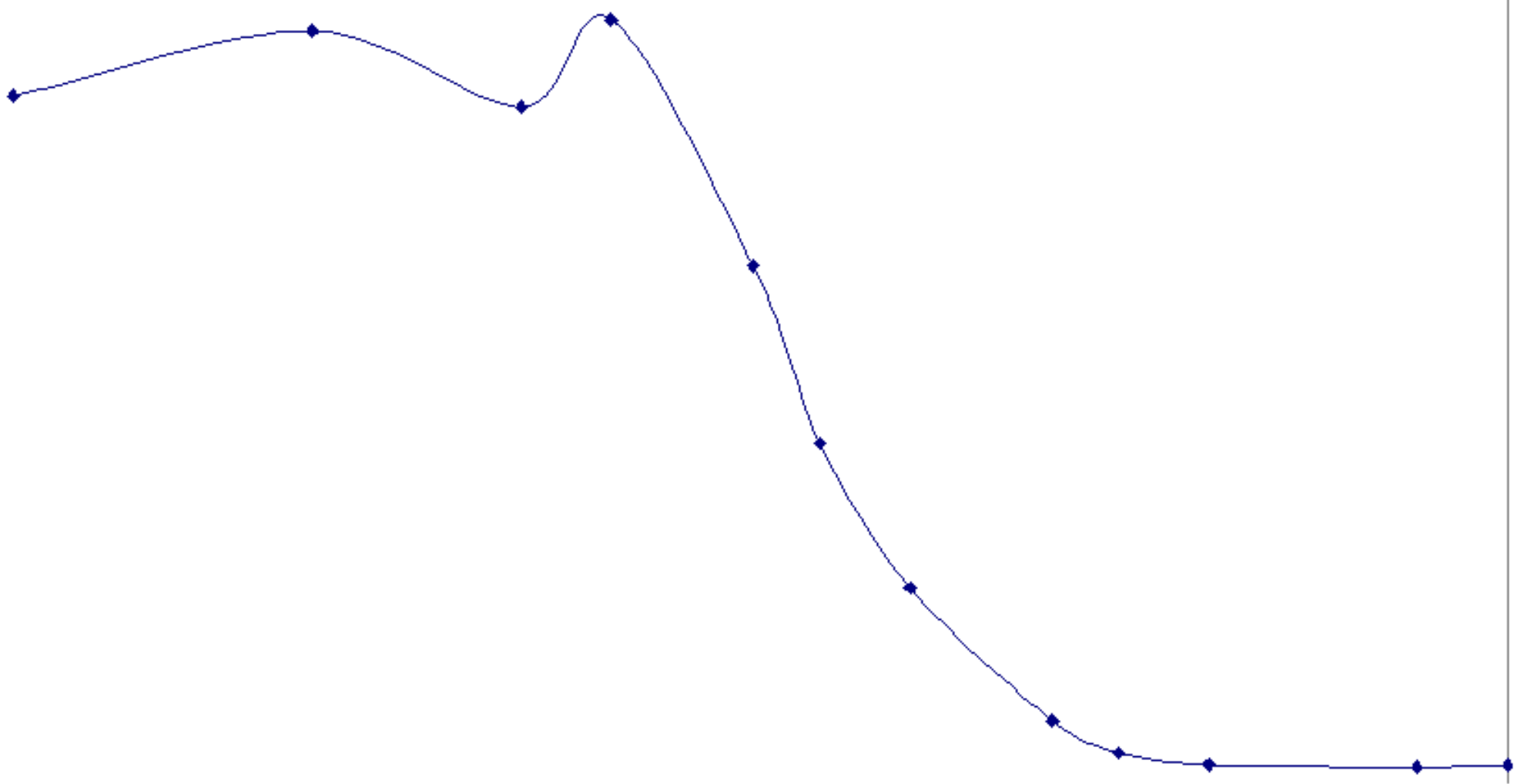




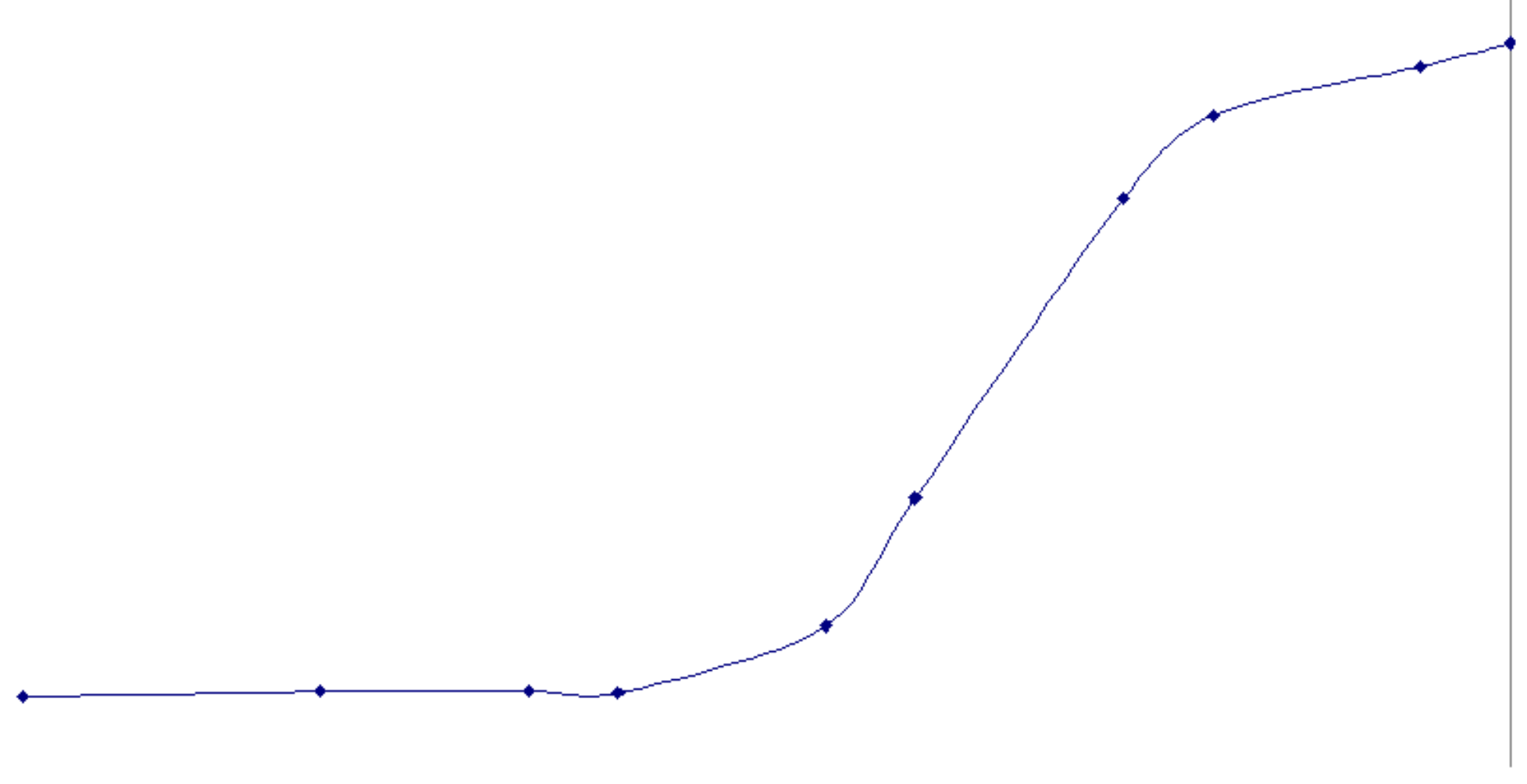




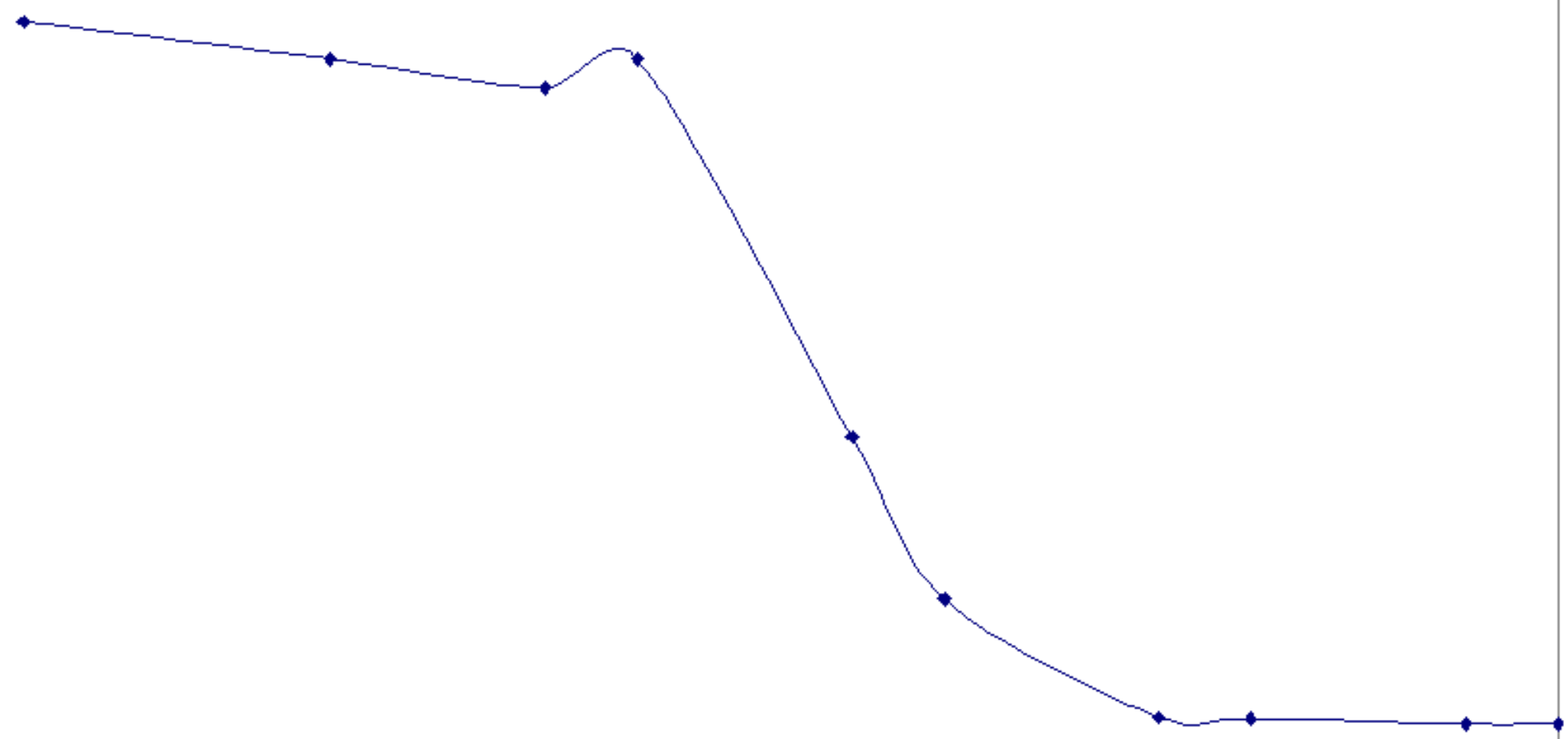



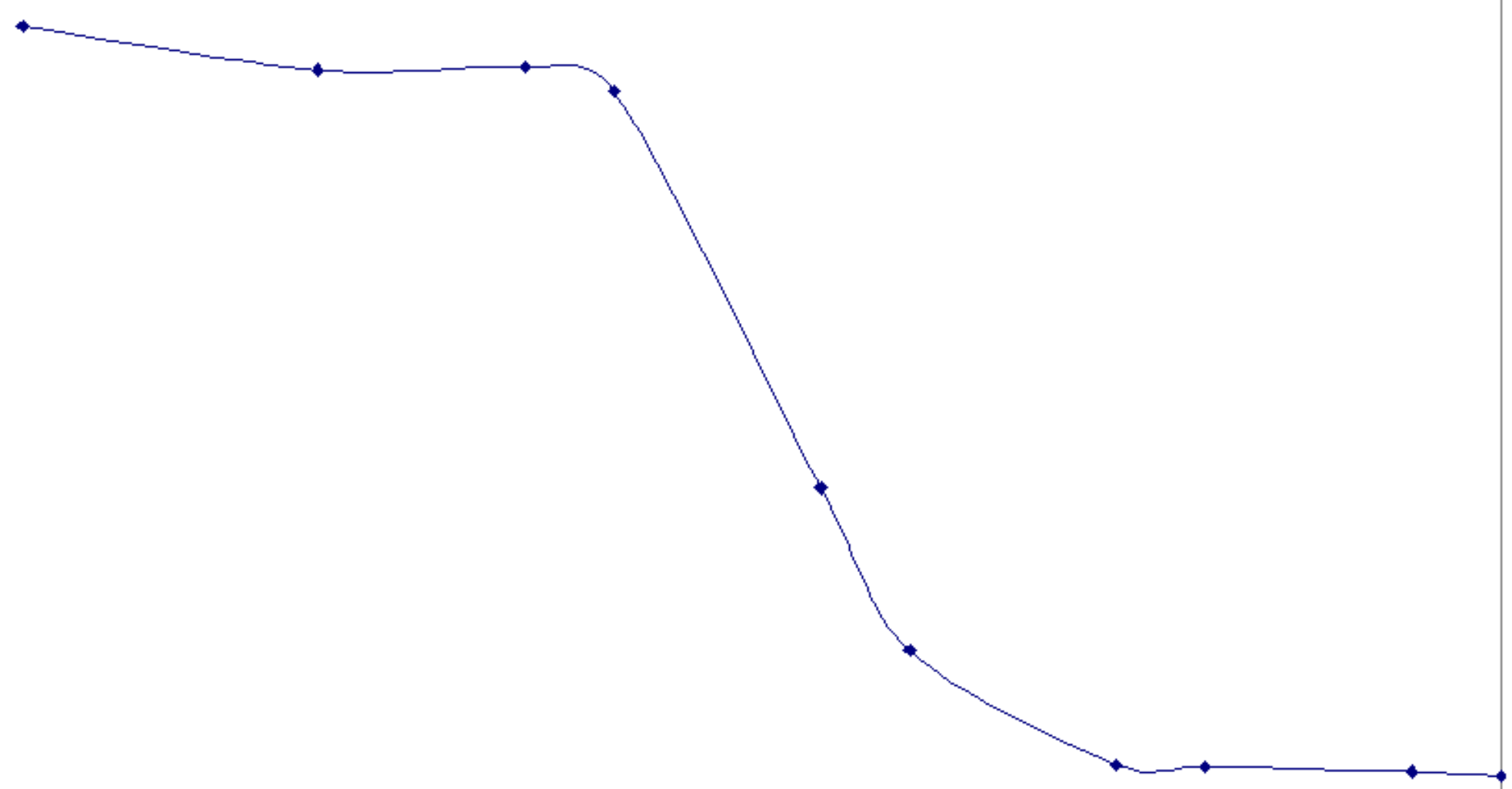

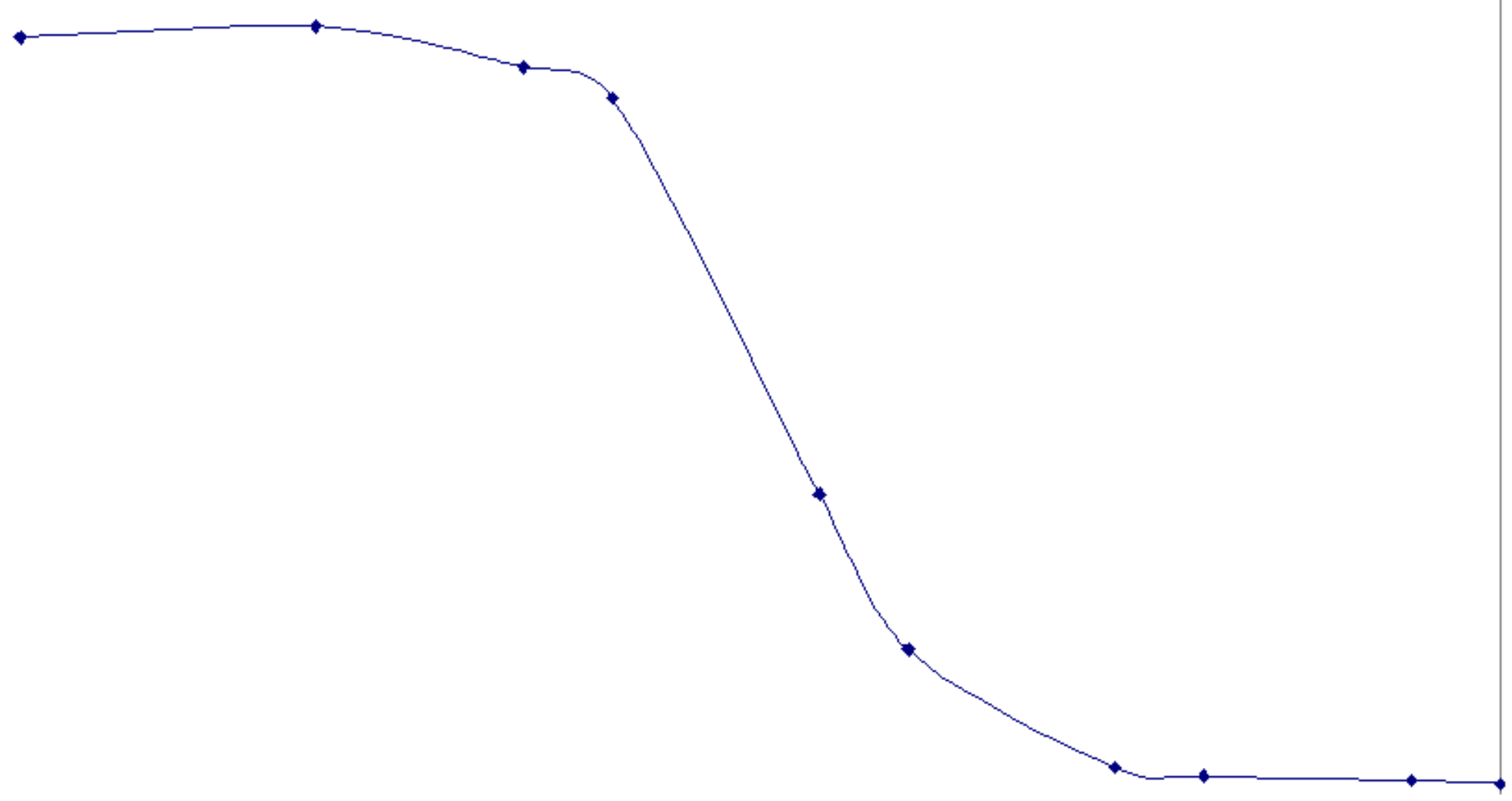

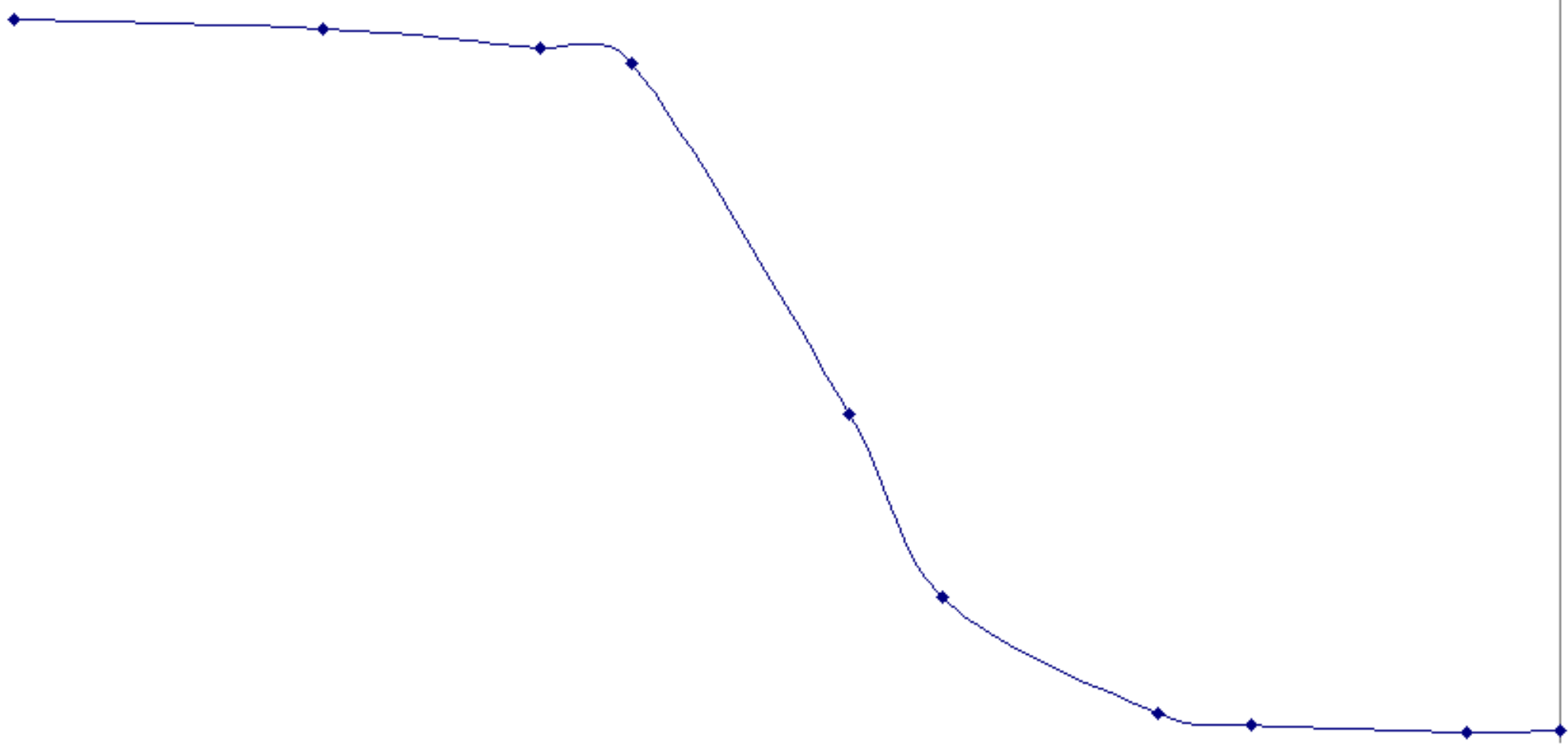


\section{Appendix C}

Calibration Curves for Kester 197 Rosin Flux on Aluminum Panels and Stainless Steel Disks 
<smiles>CCCCCCC</smiles> 

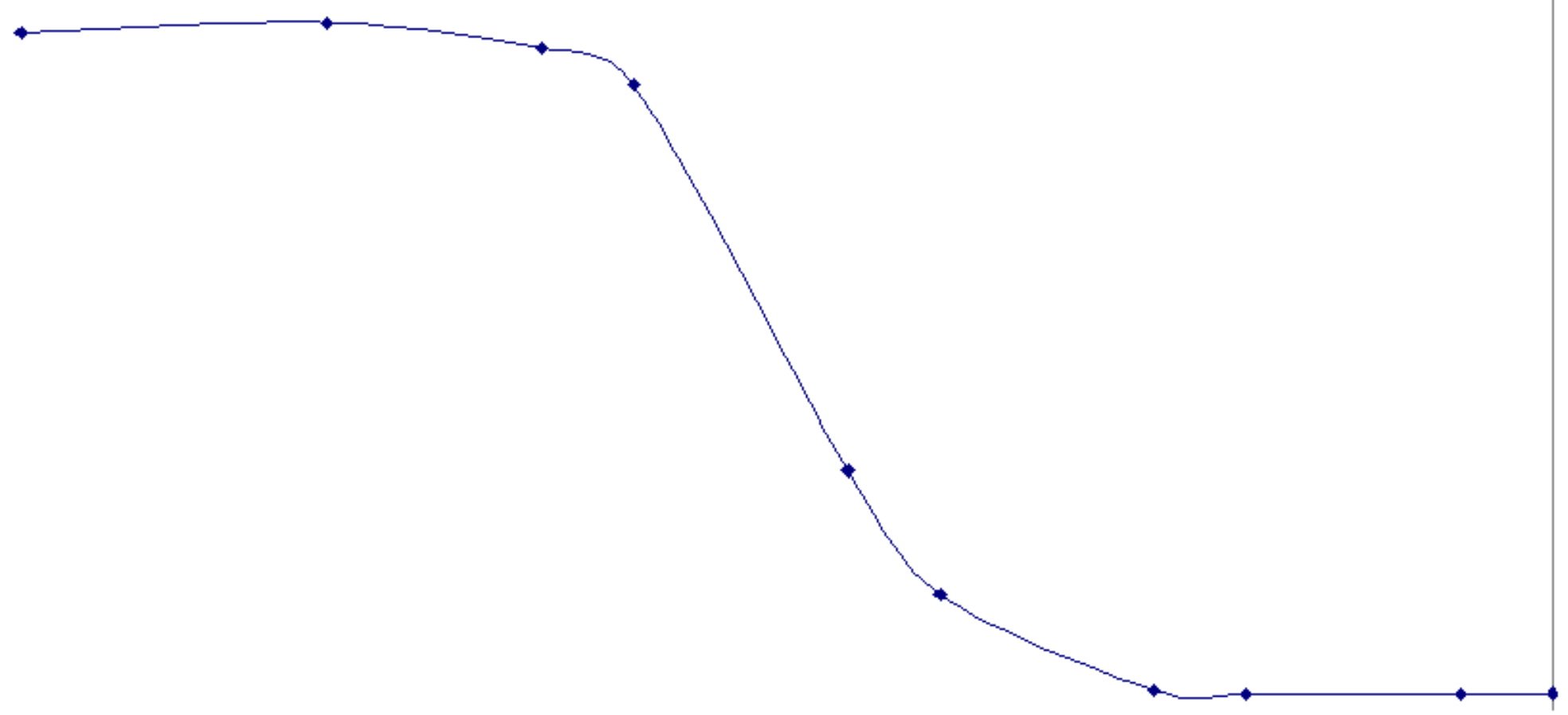


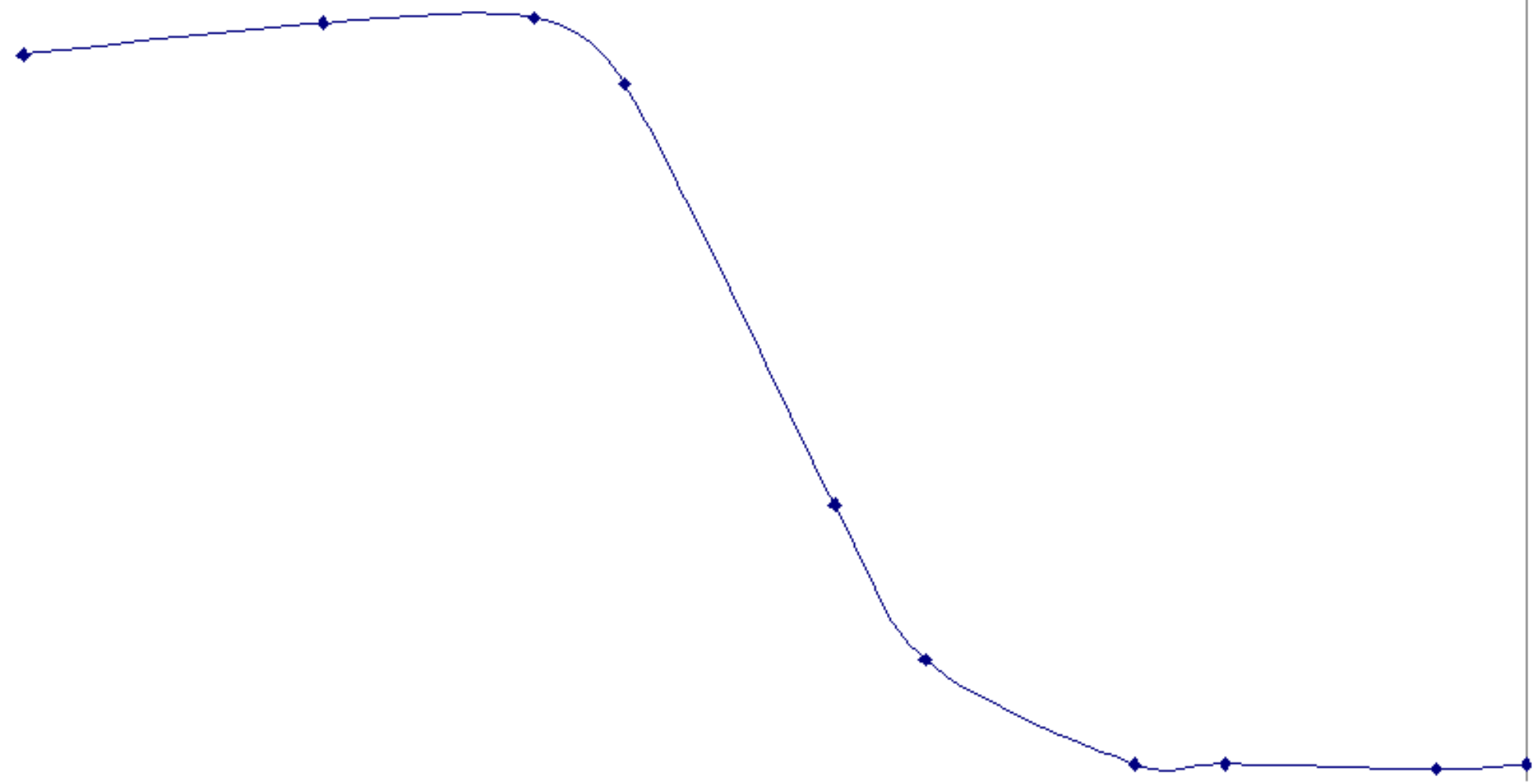




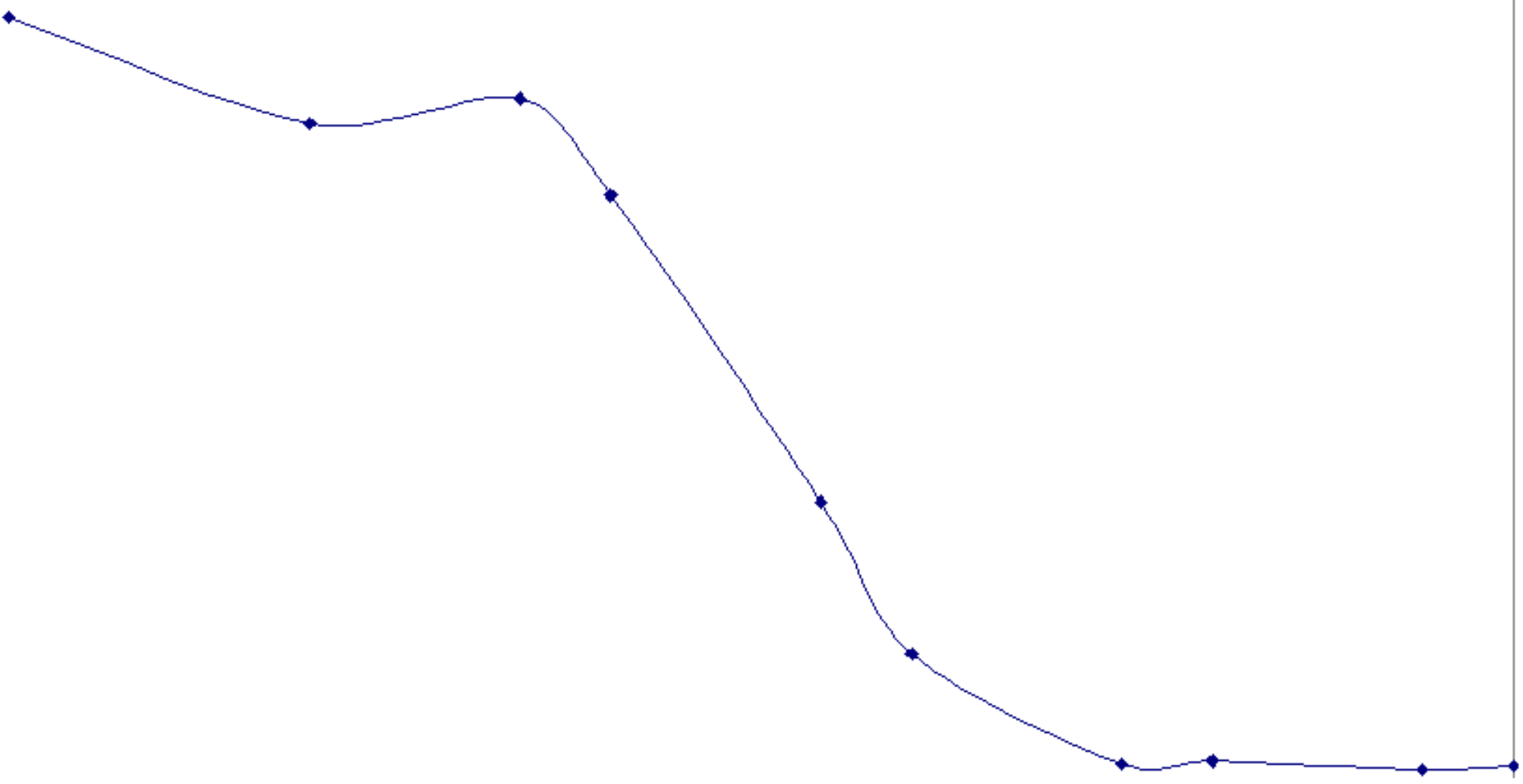




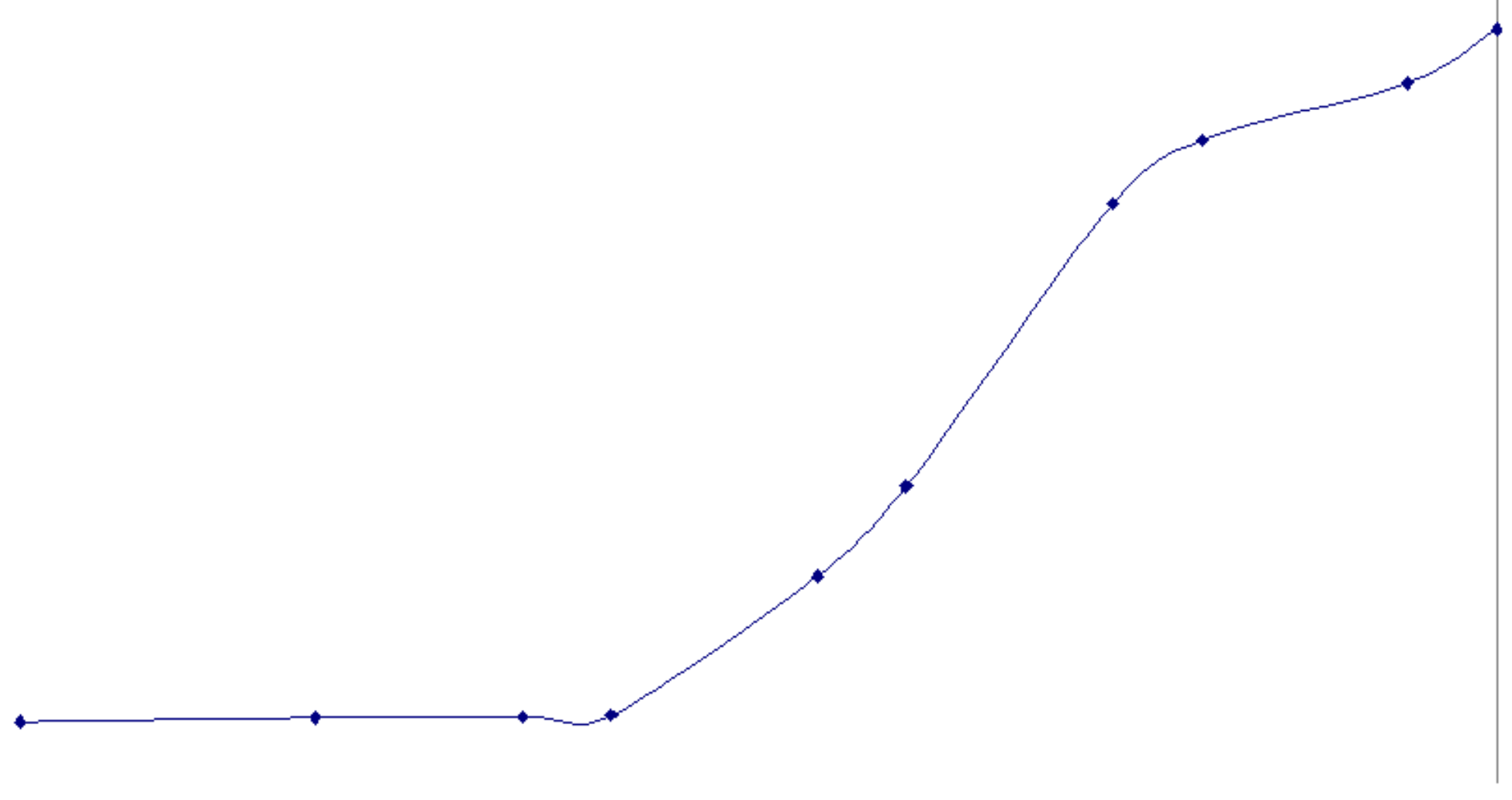



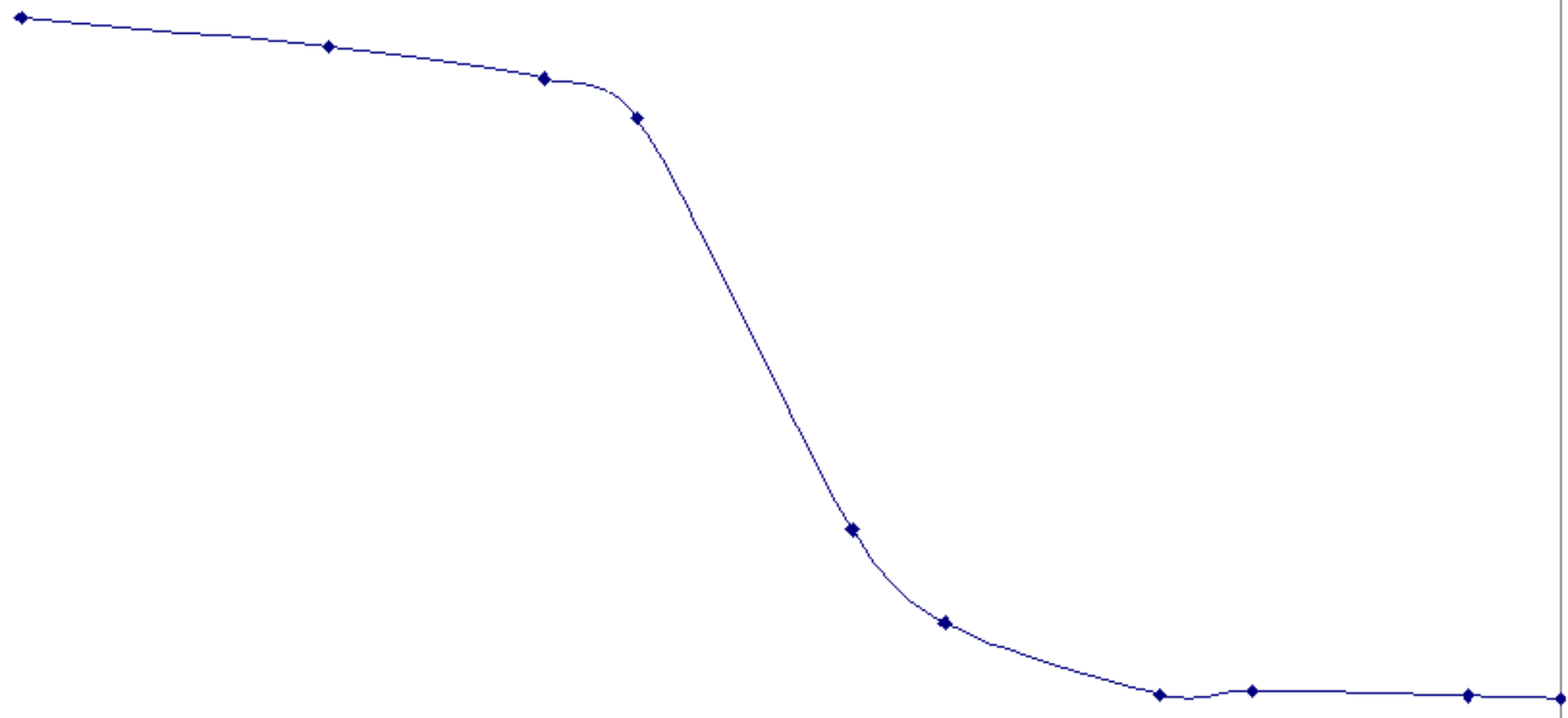
<smiles>CCCCCC</smiles> 
FY98 Annual Report

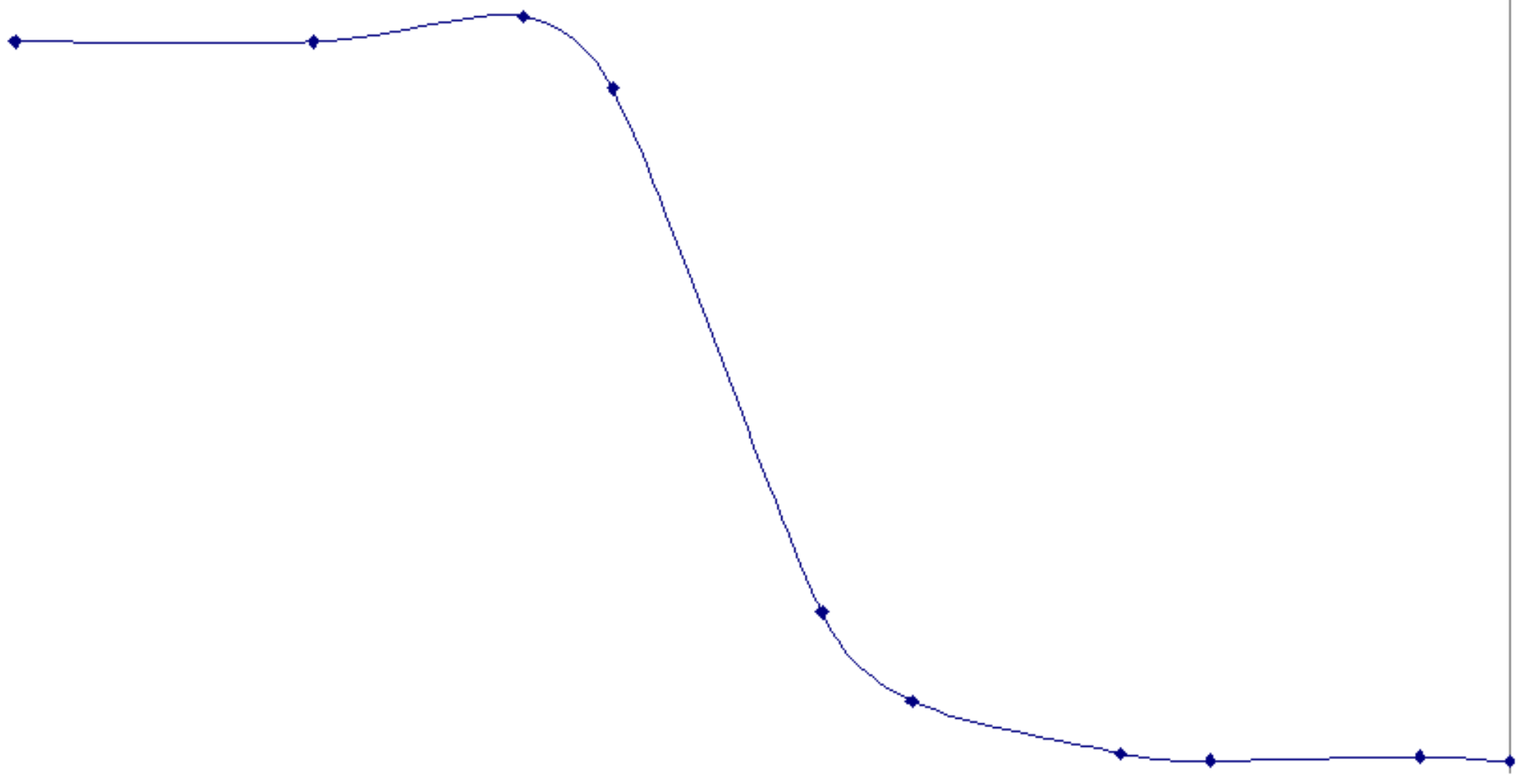

file:///C//ManualConversion/HTML/6792benk.htm (49 of 250)4/9/2004 4:05:24 AM 


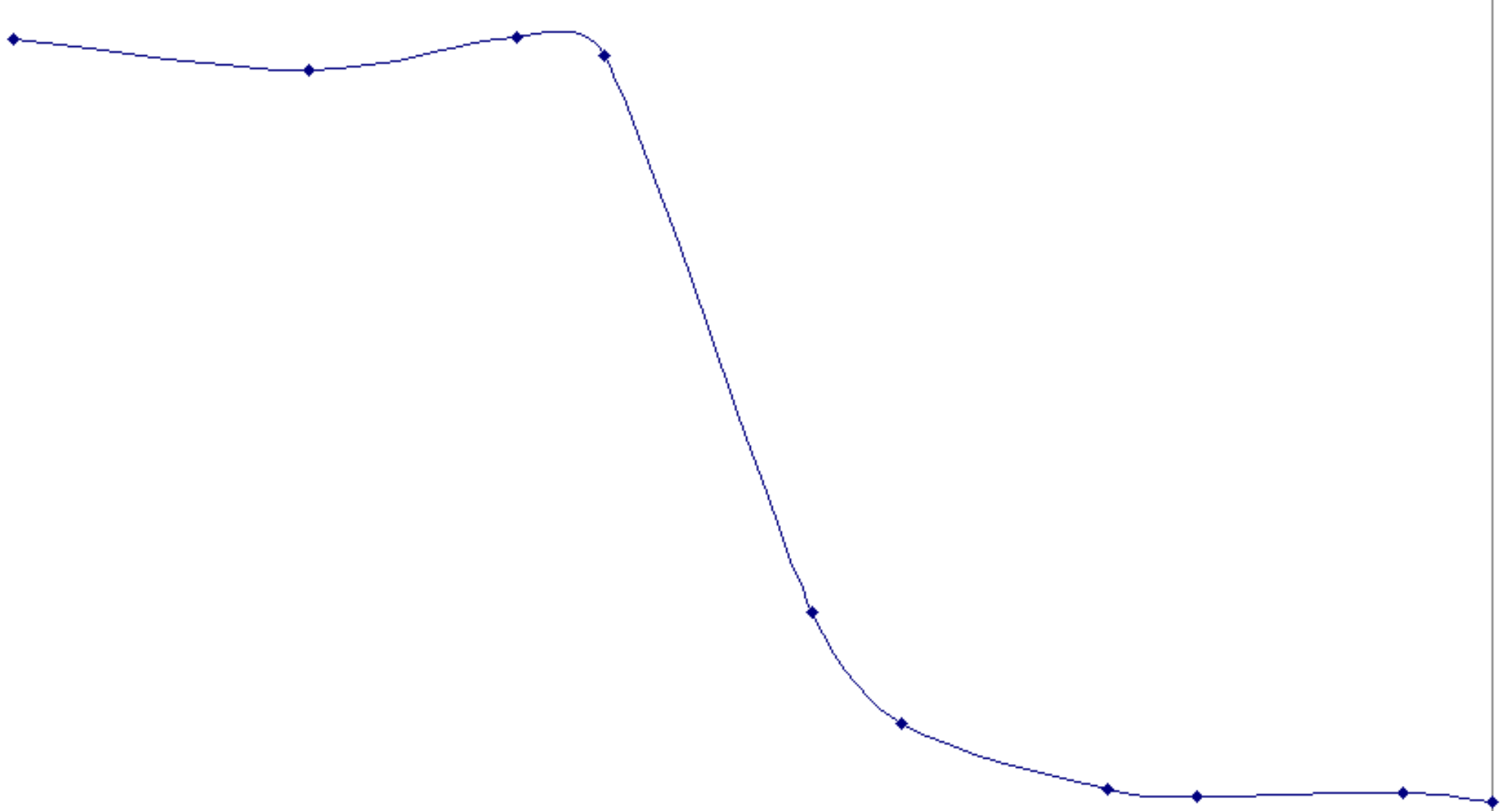




\section{Appendix D}

Calibration Curves for Kester 1544 Rosin Flux on Aluminum Panels and Stainless Steel Disks

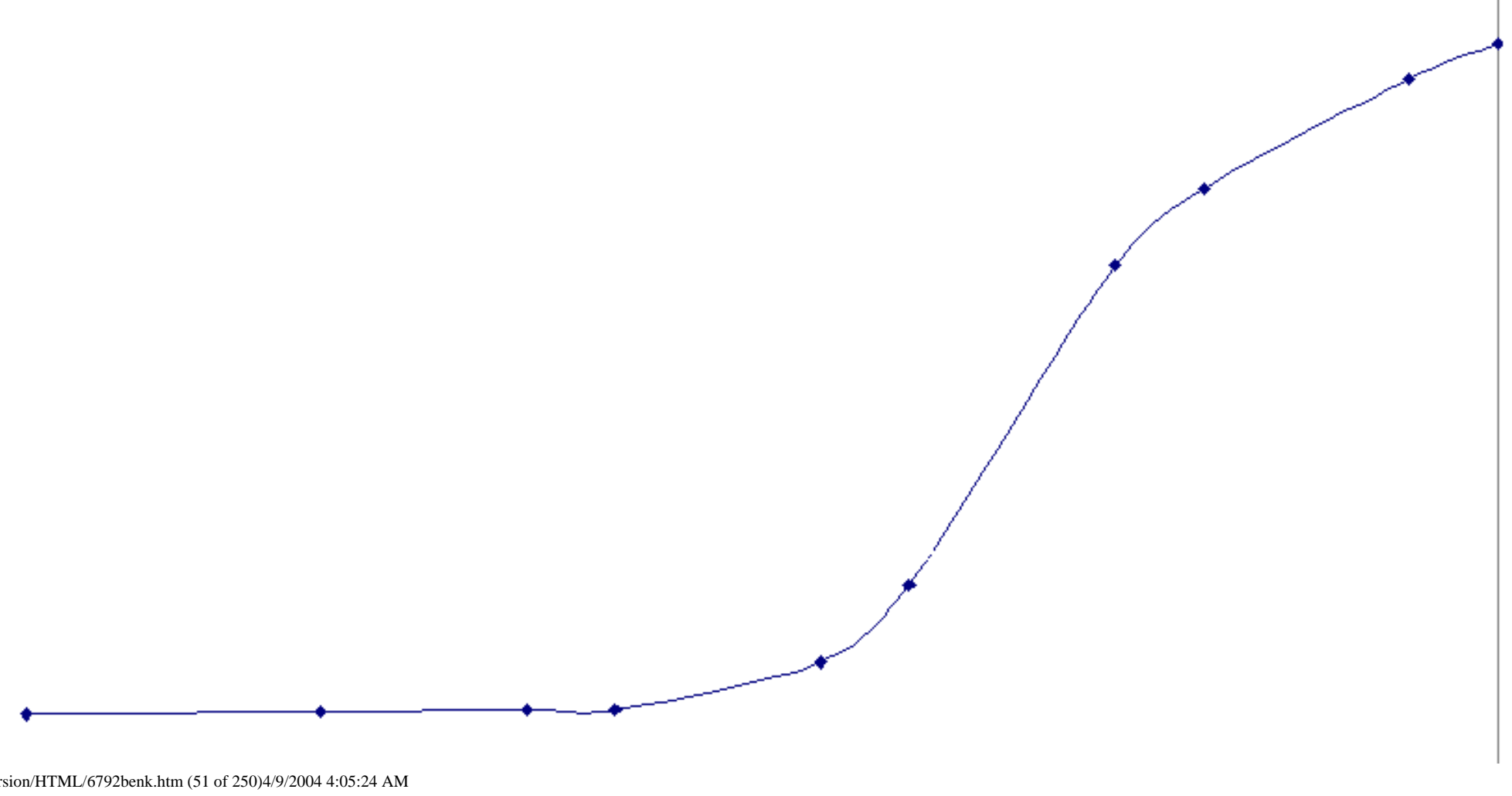



<smiles>CCCCCC</smiles> 


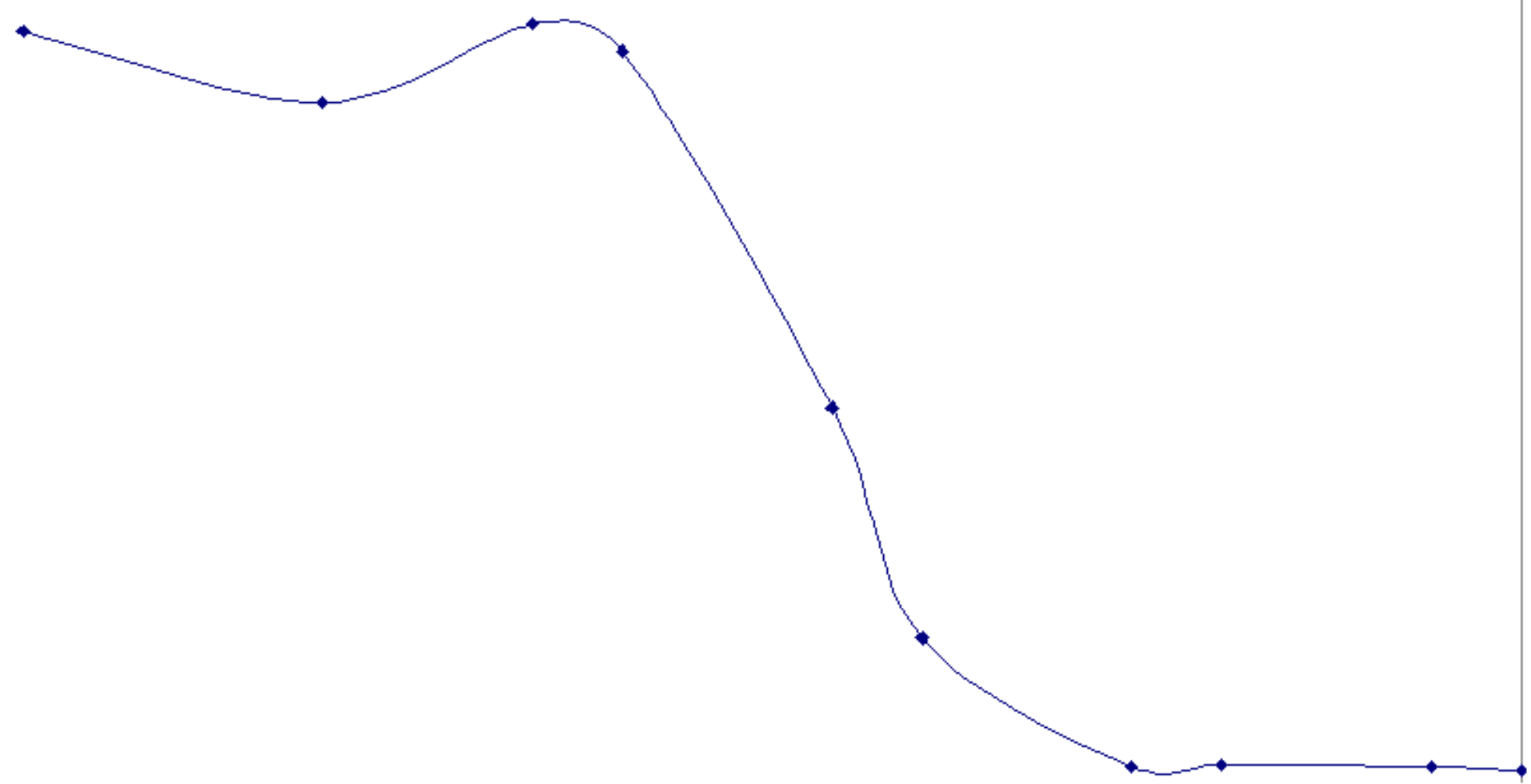




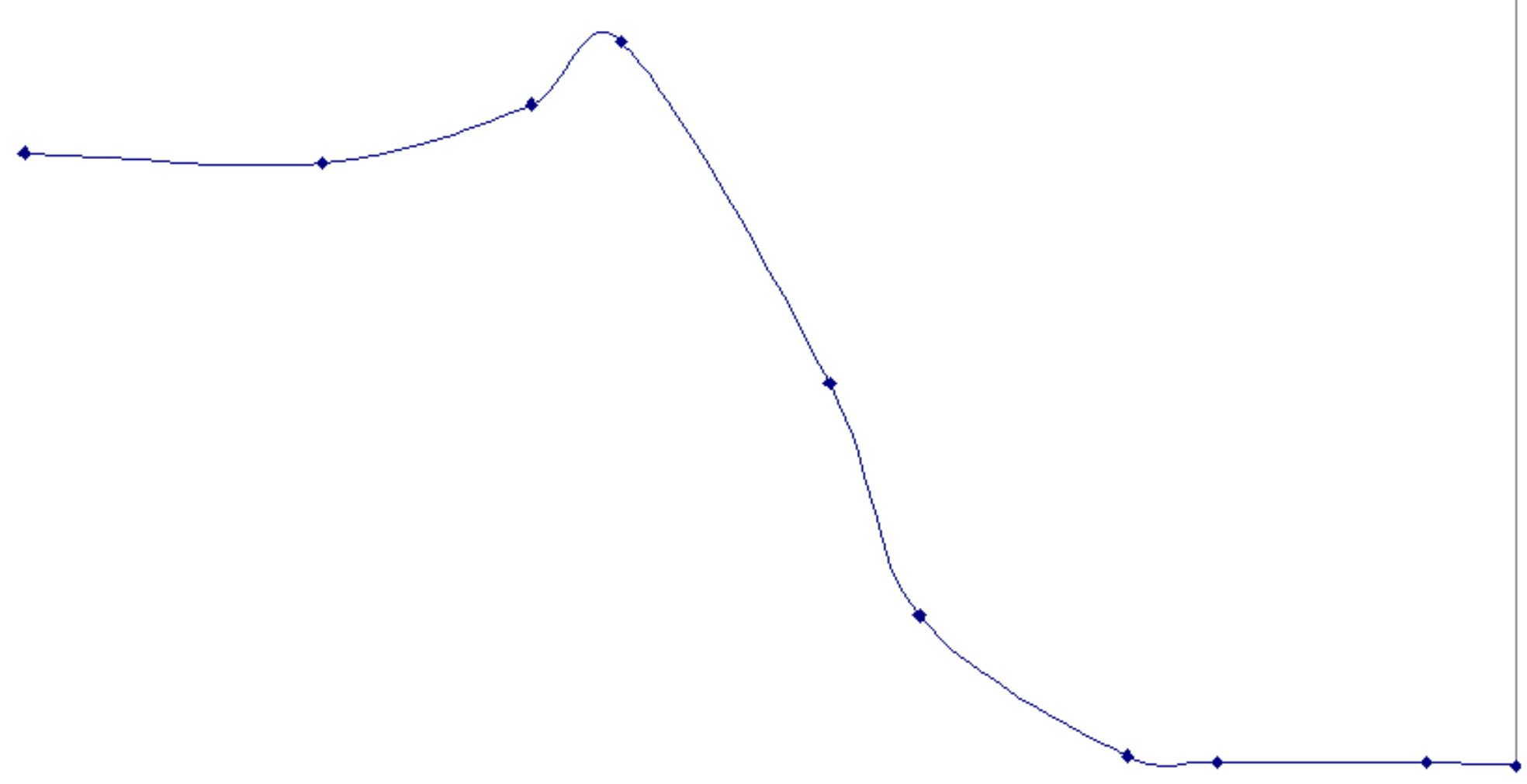




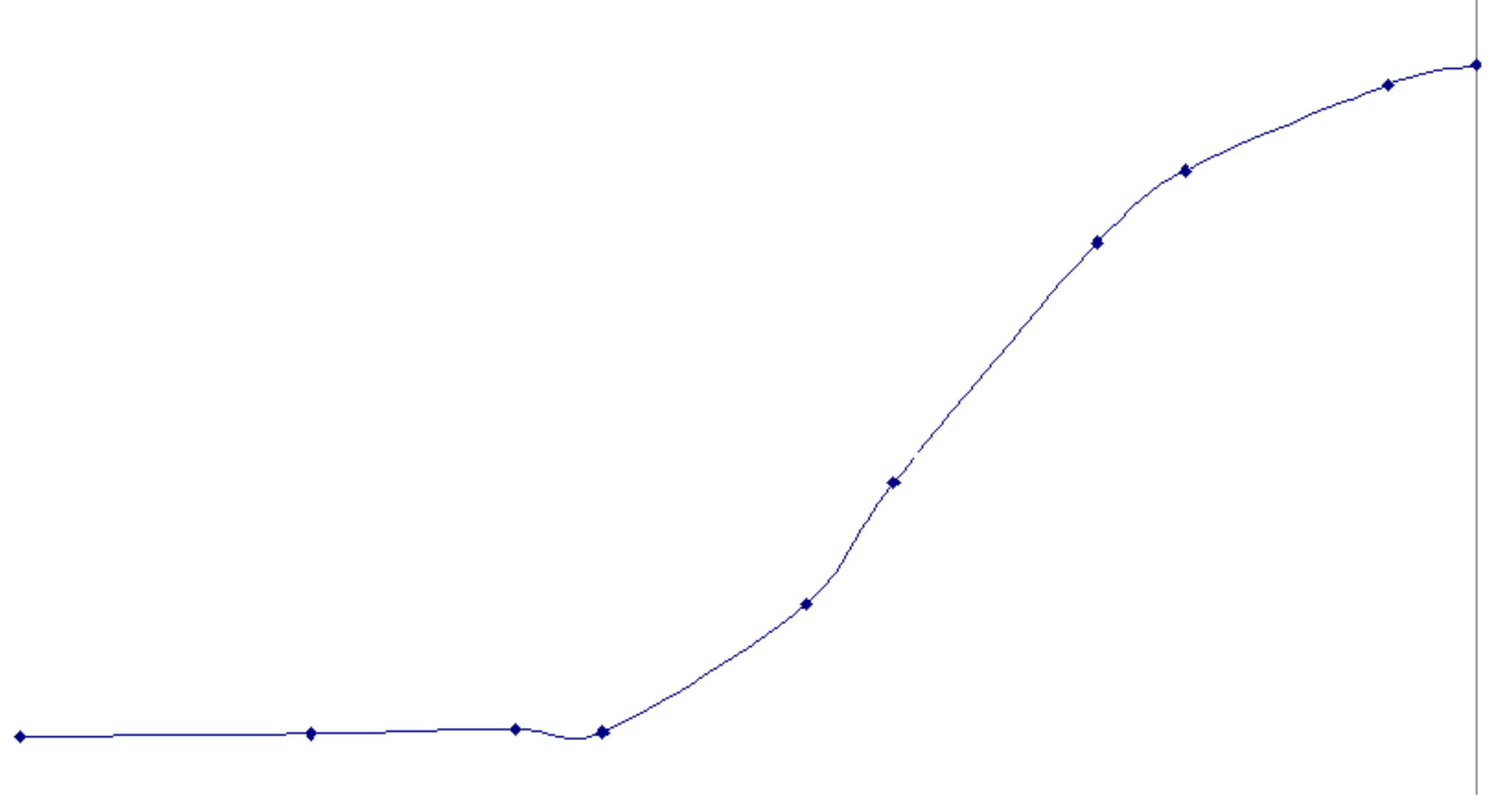




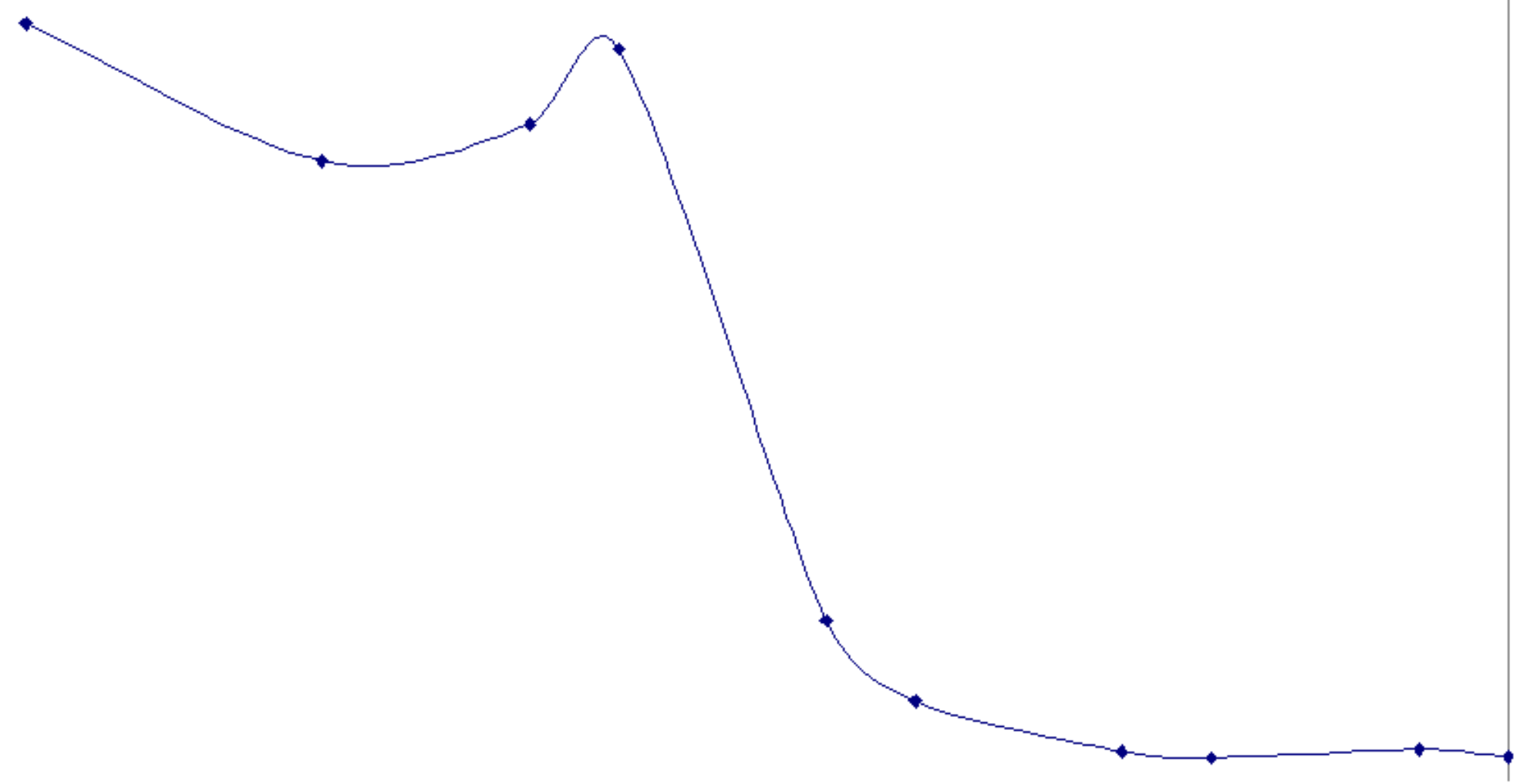


<smiles>CCCCC</smiles> 
FY98 Annual Report

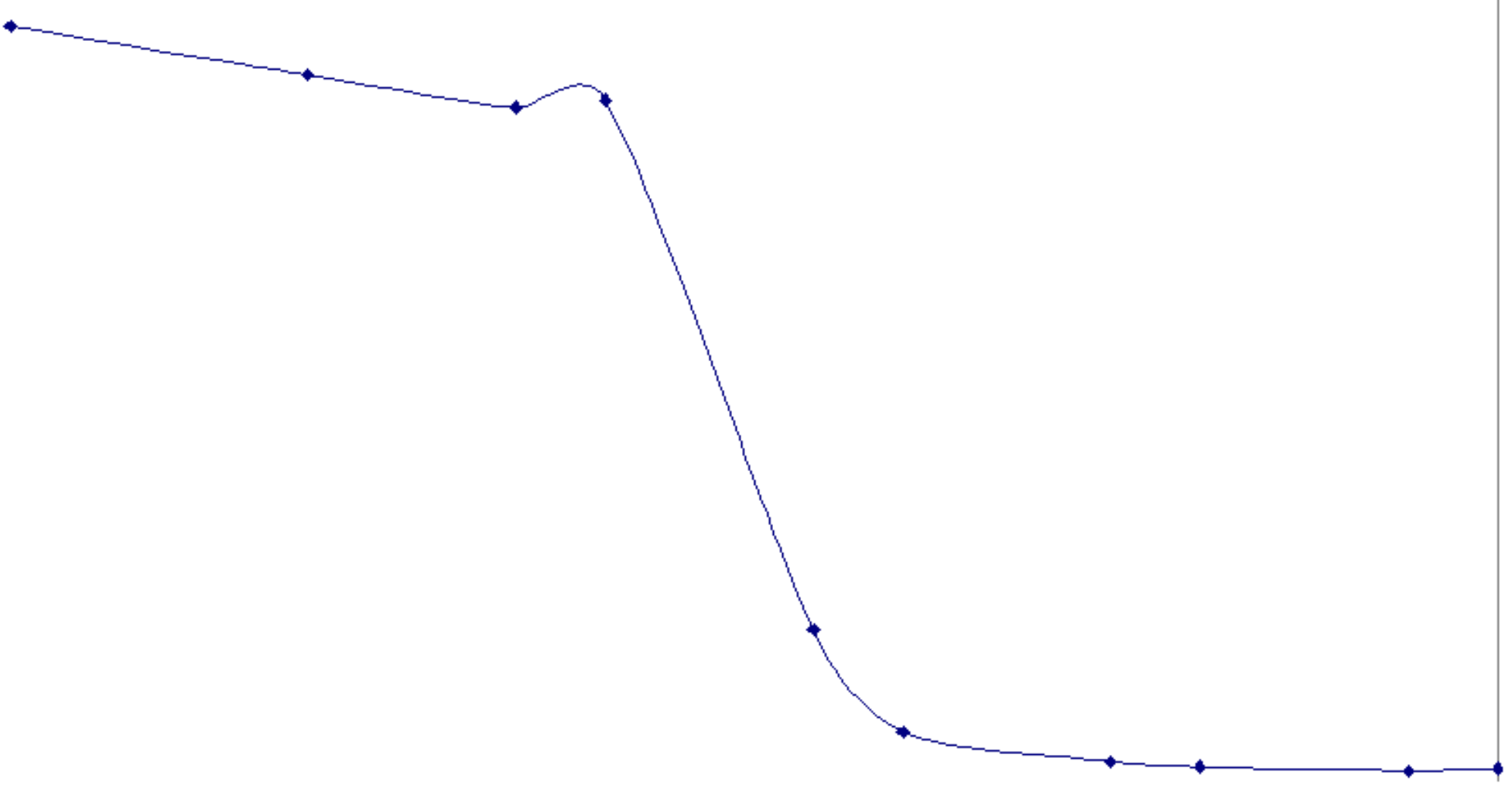

file://C|/ManualConversion/HTML/6792benk.htm (59 of 250)4/9/2004 4:05:24 AM 


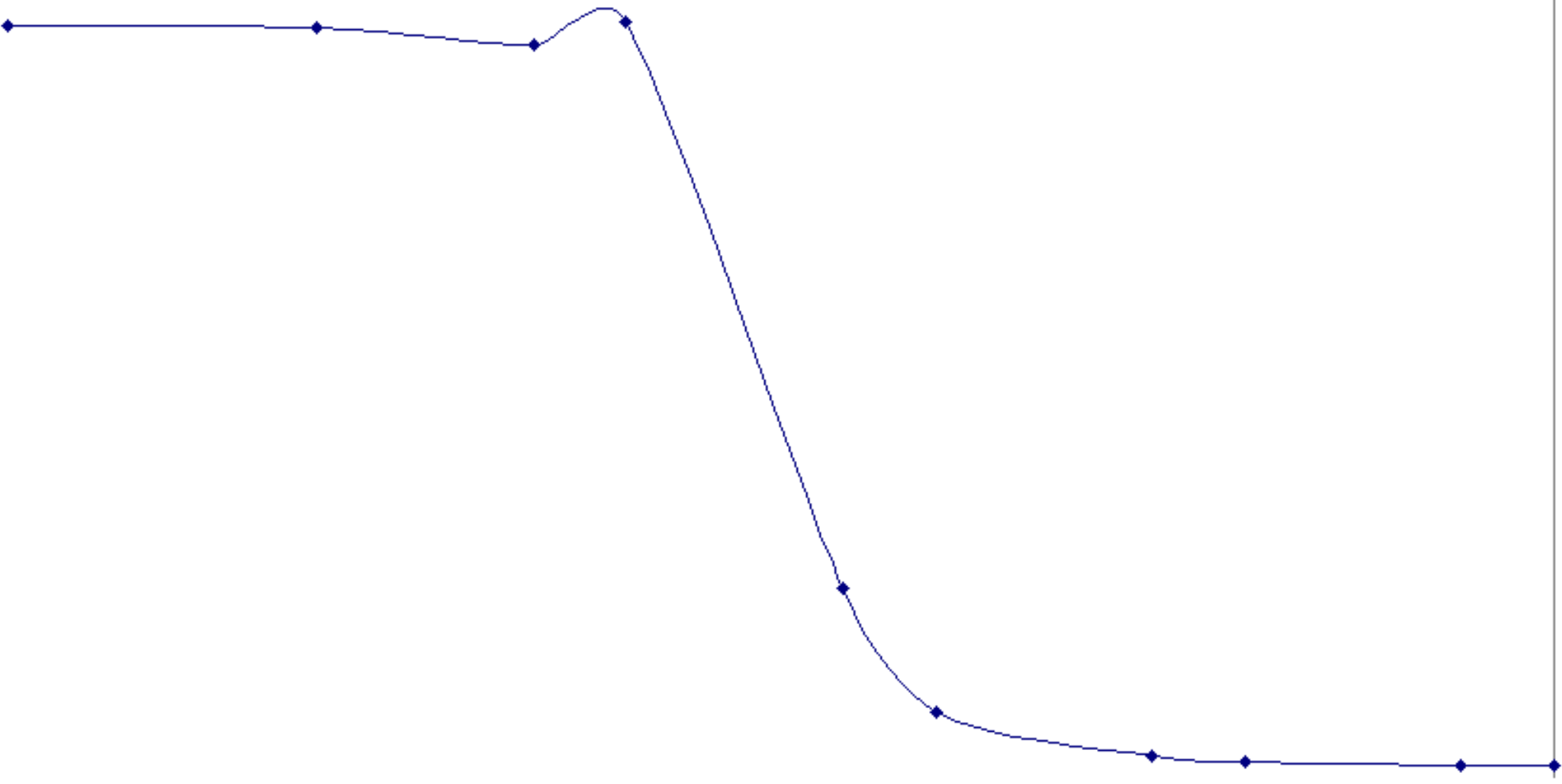




\section{Appendix E}

\section{Calibration Curves for WD-40 Oil}

on Aluminum Panels and Stainless Steel Disks 
FY98 Annual Report

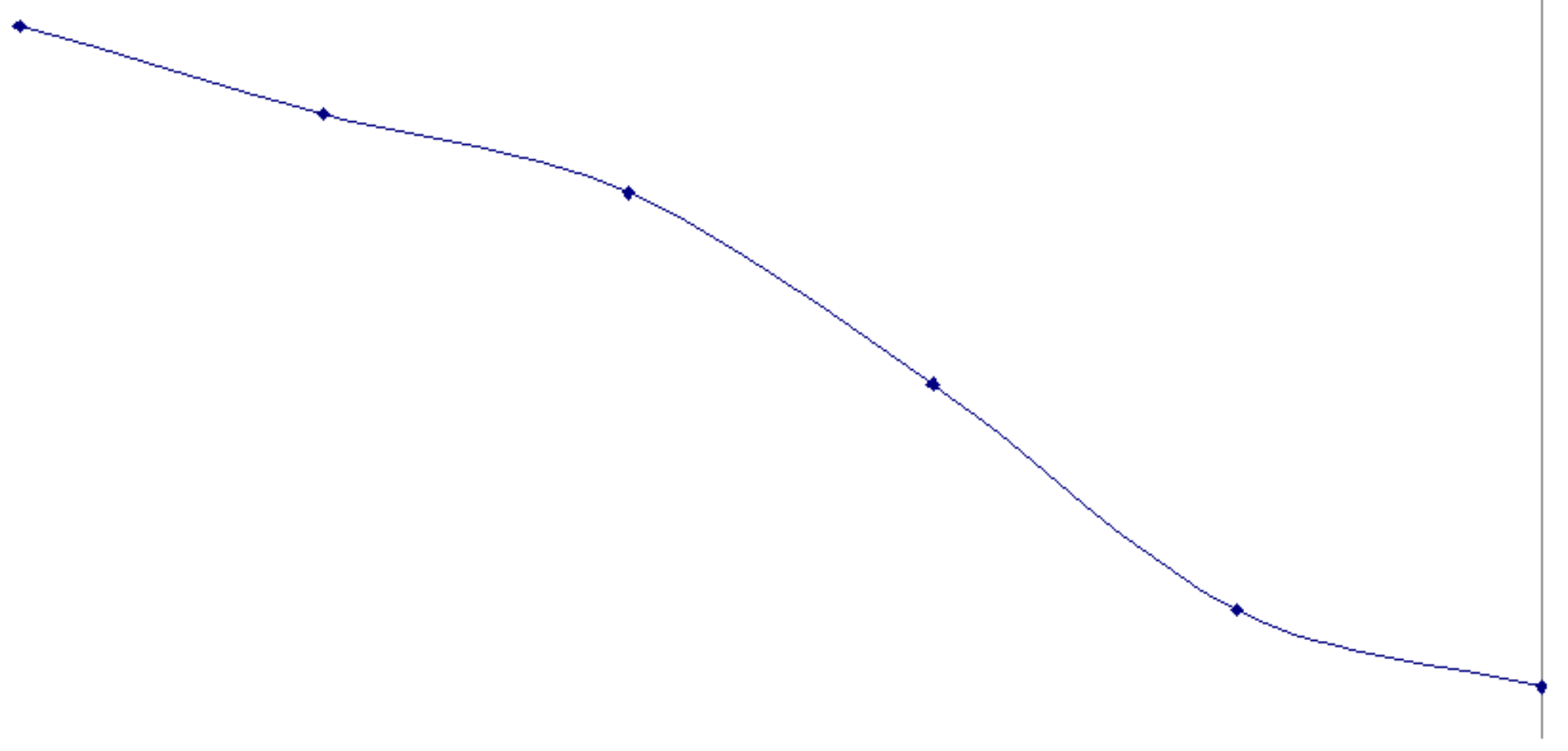

file:///C/ManualConversion/HTML/6792benk.htm (62 of 250)4/9/2004 4:05:24 AM 

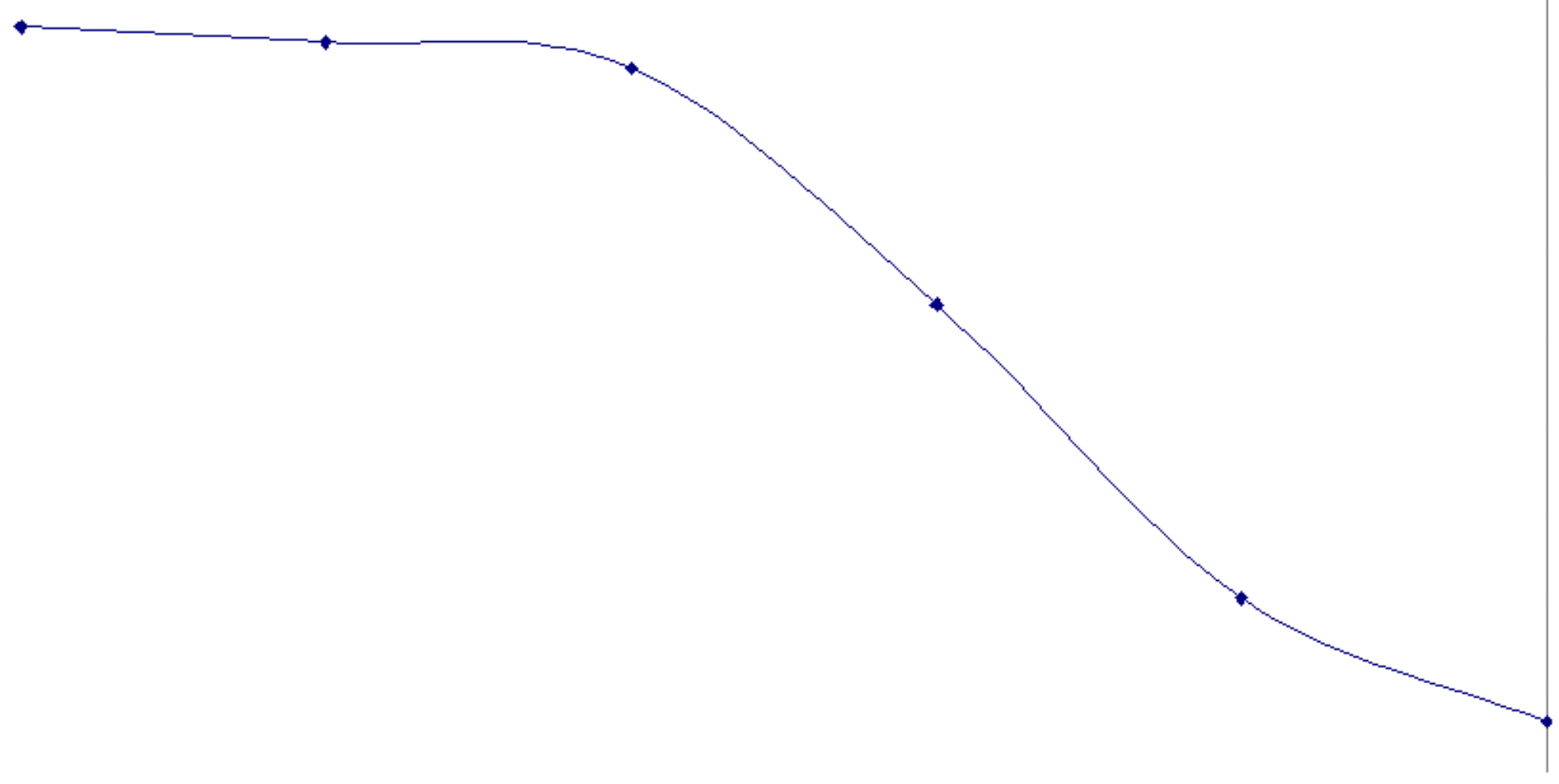

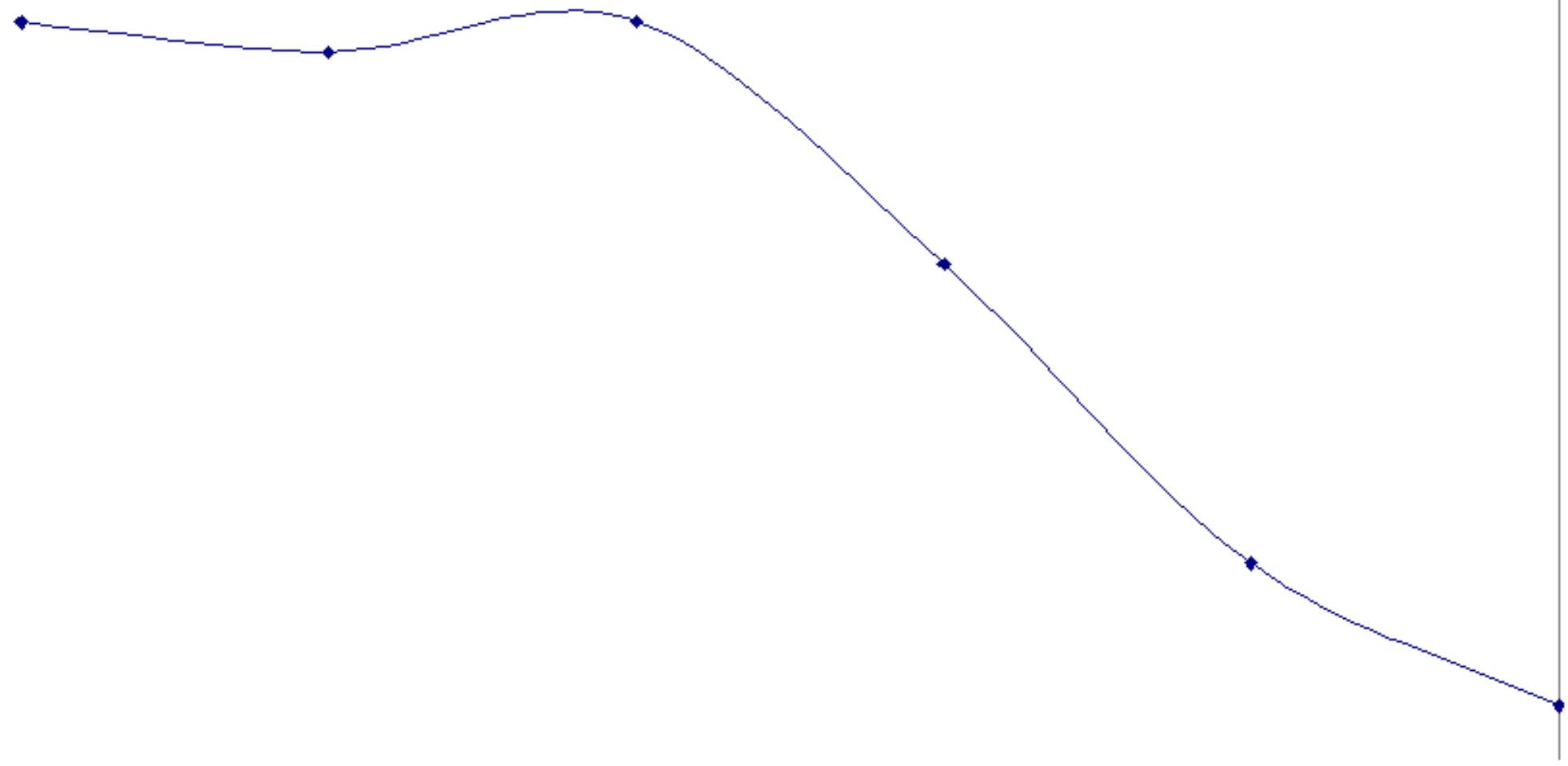


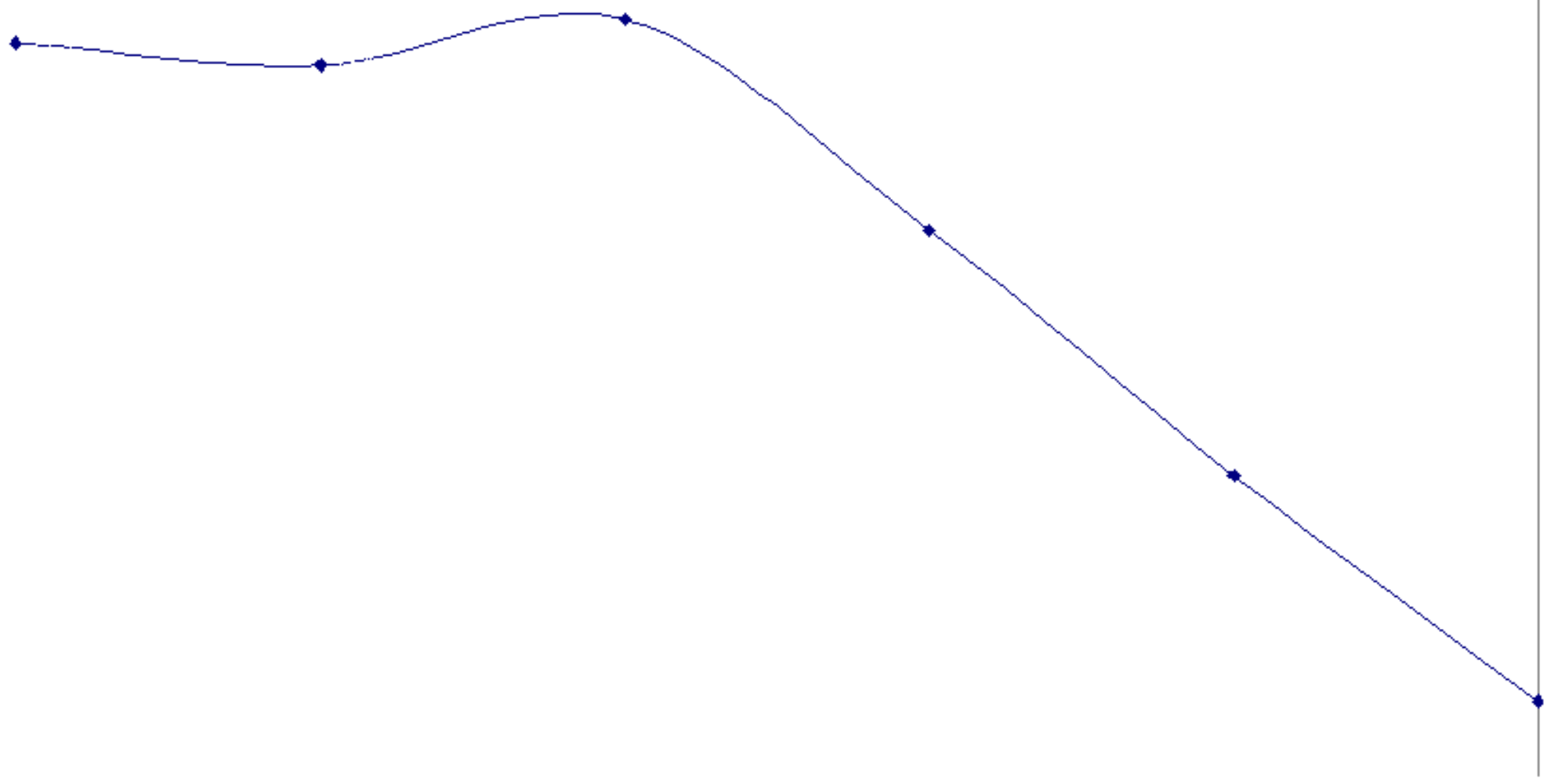


<smiles>CCC</smiles> 

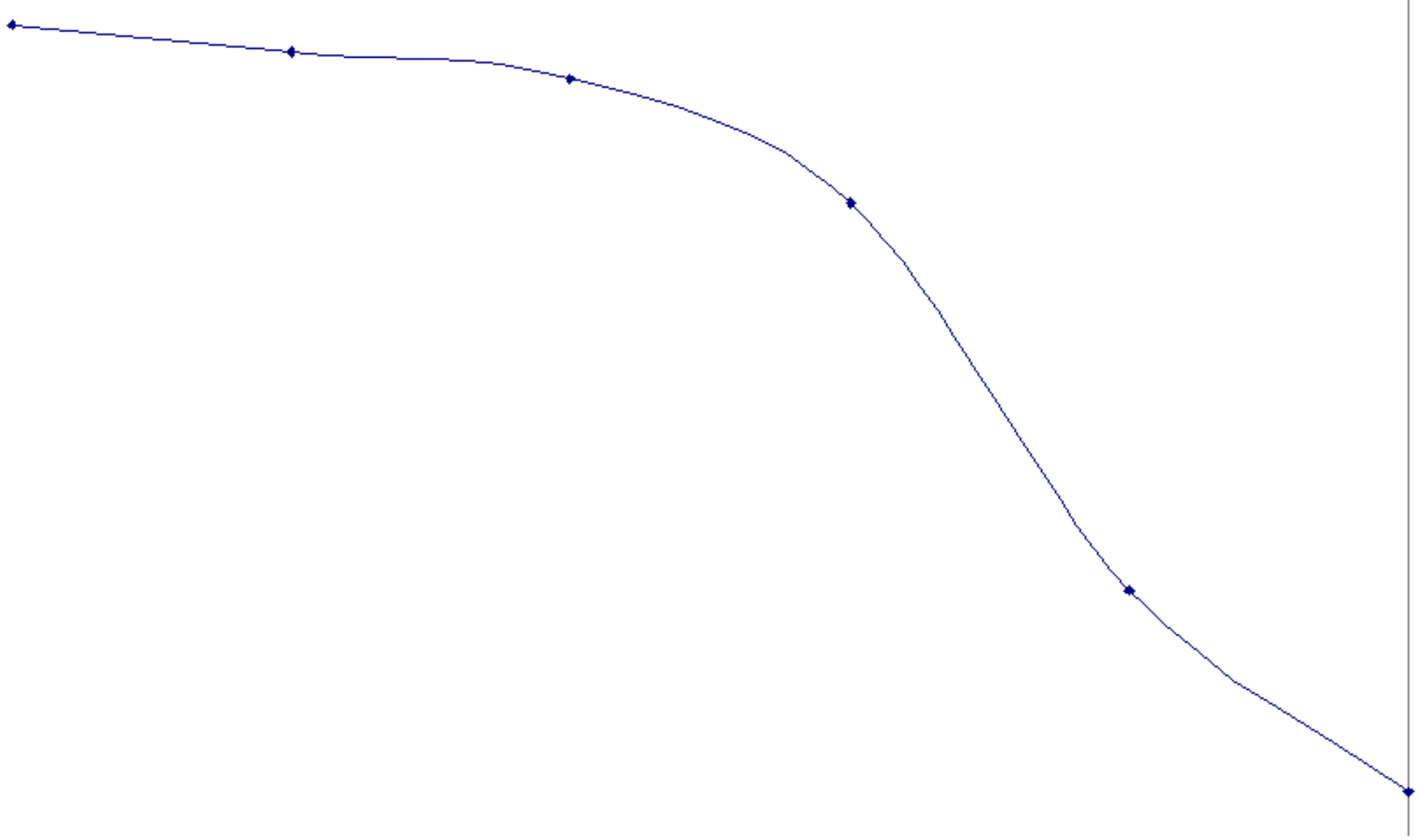


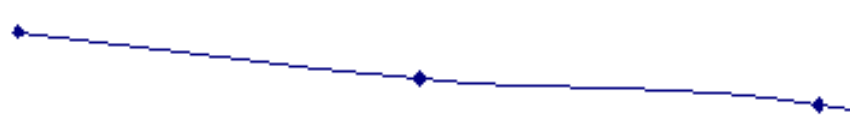




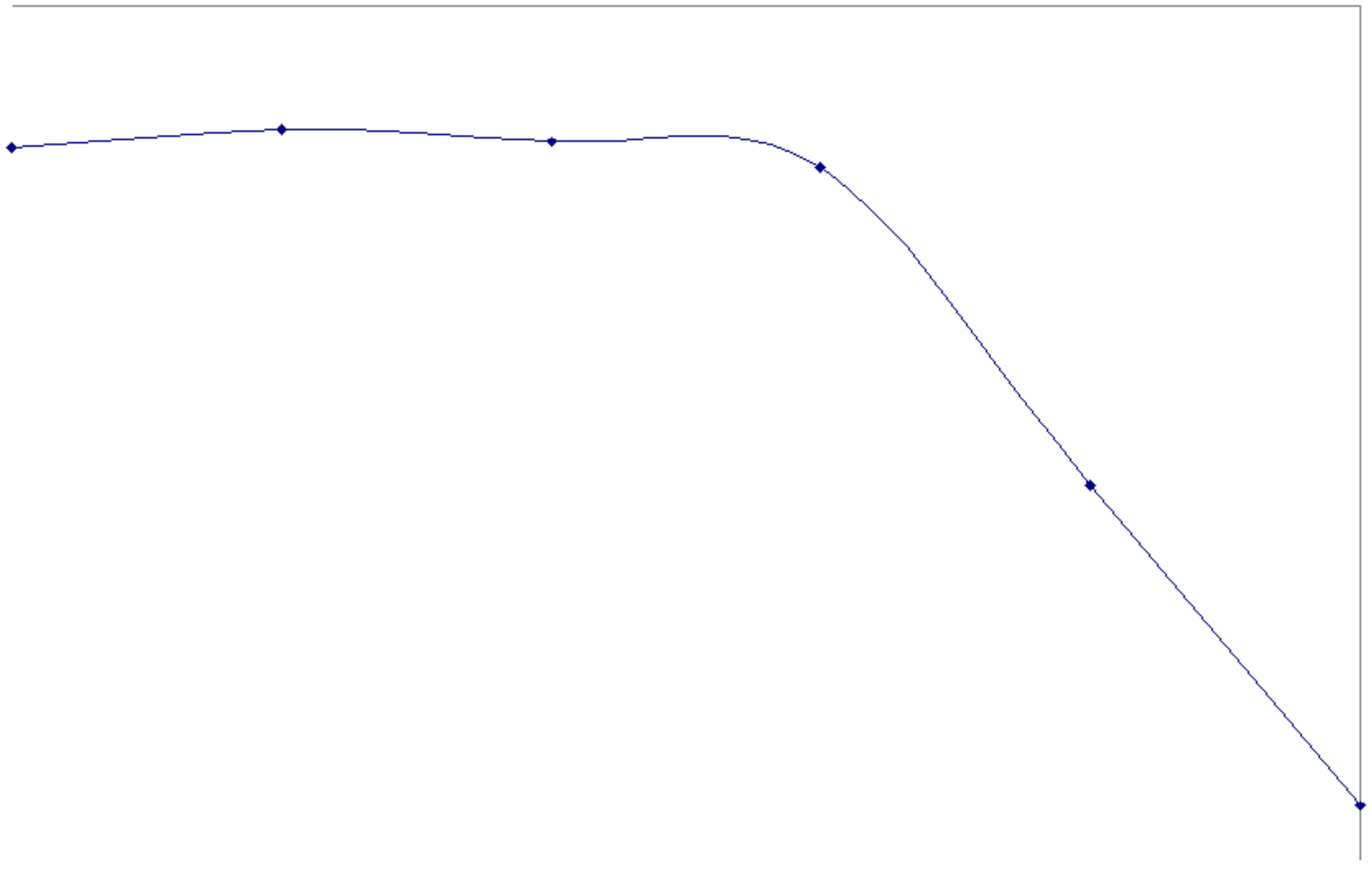




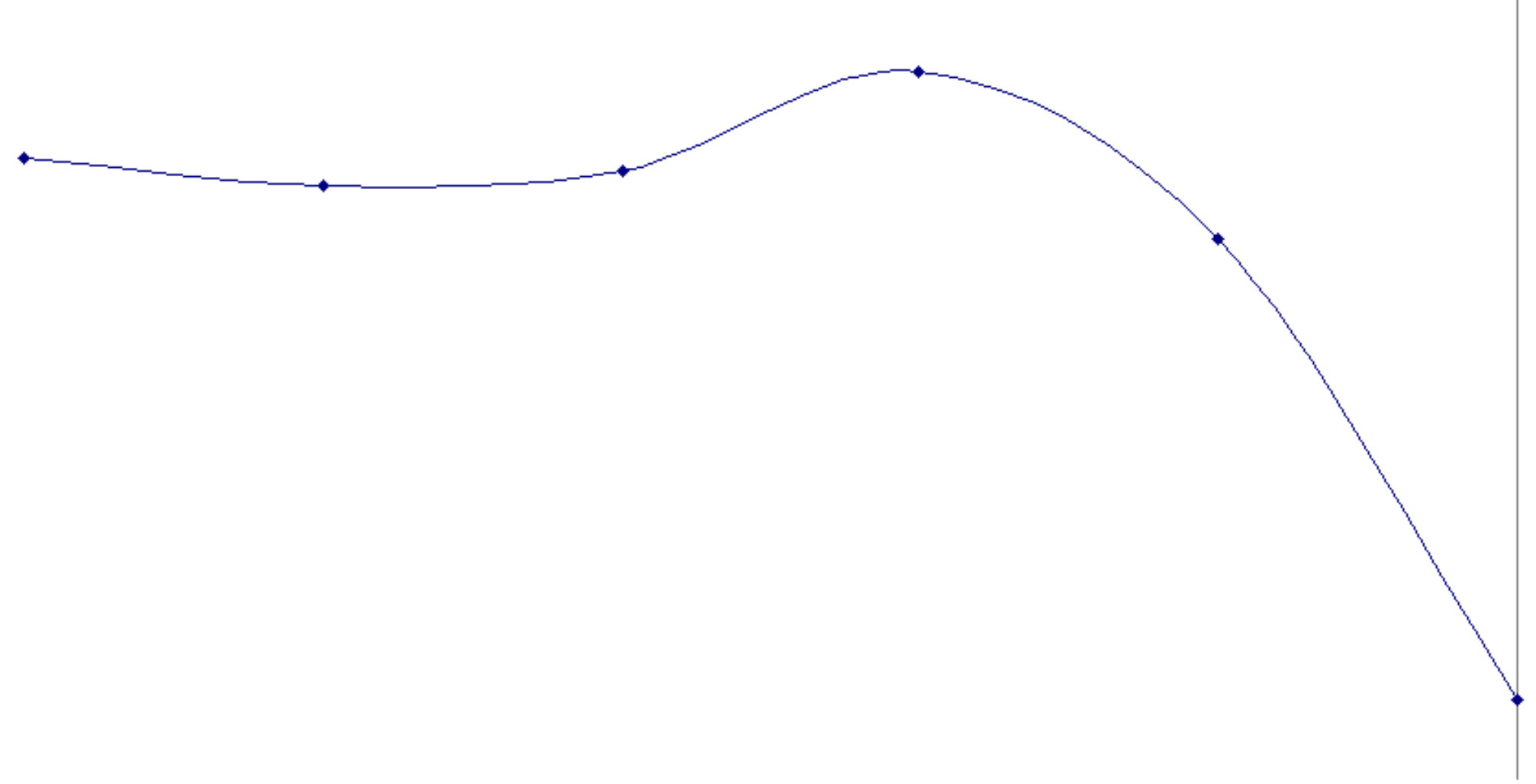


Appendix F

Calibration Curves for Apiezon Grease M

on Aluminum Panels and Stainless Steel Disks

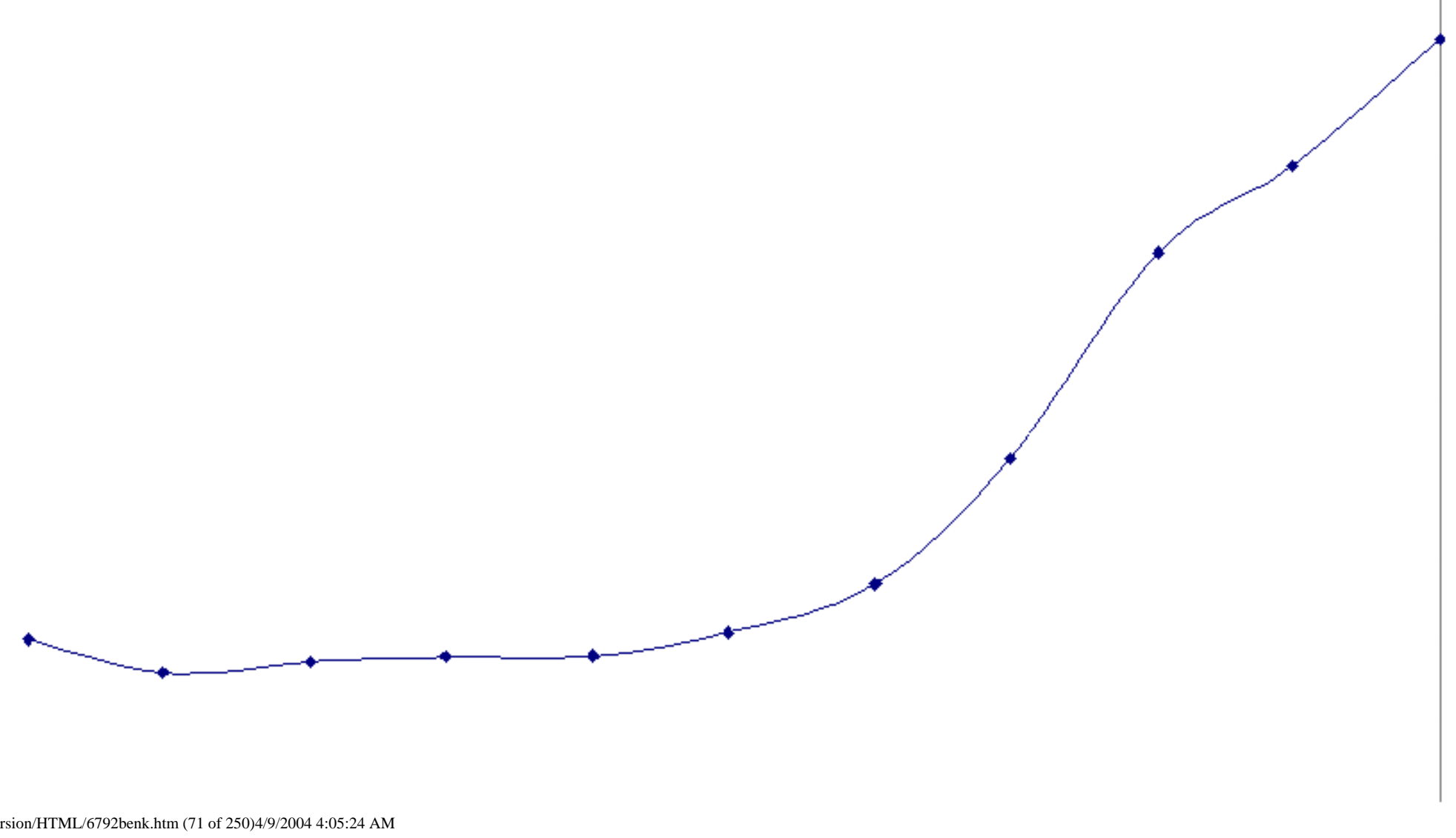


$+1$
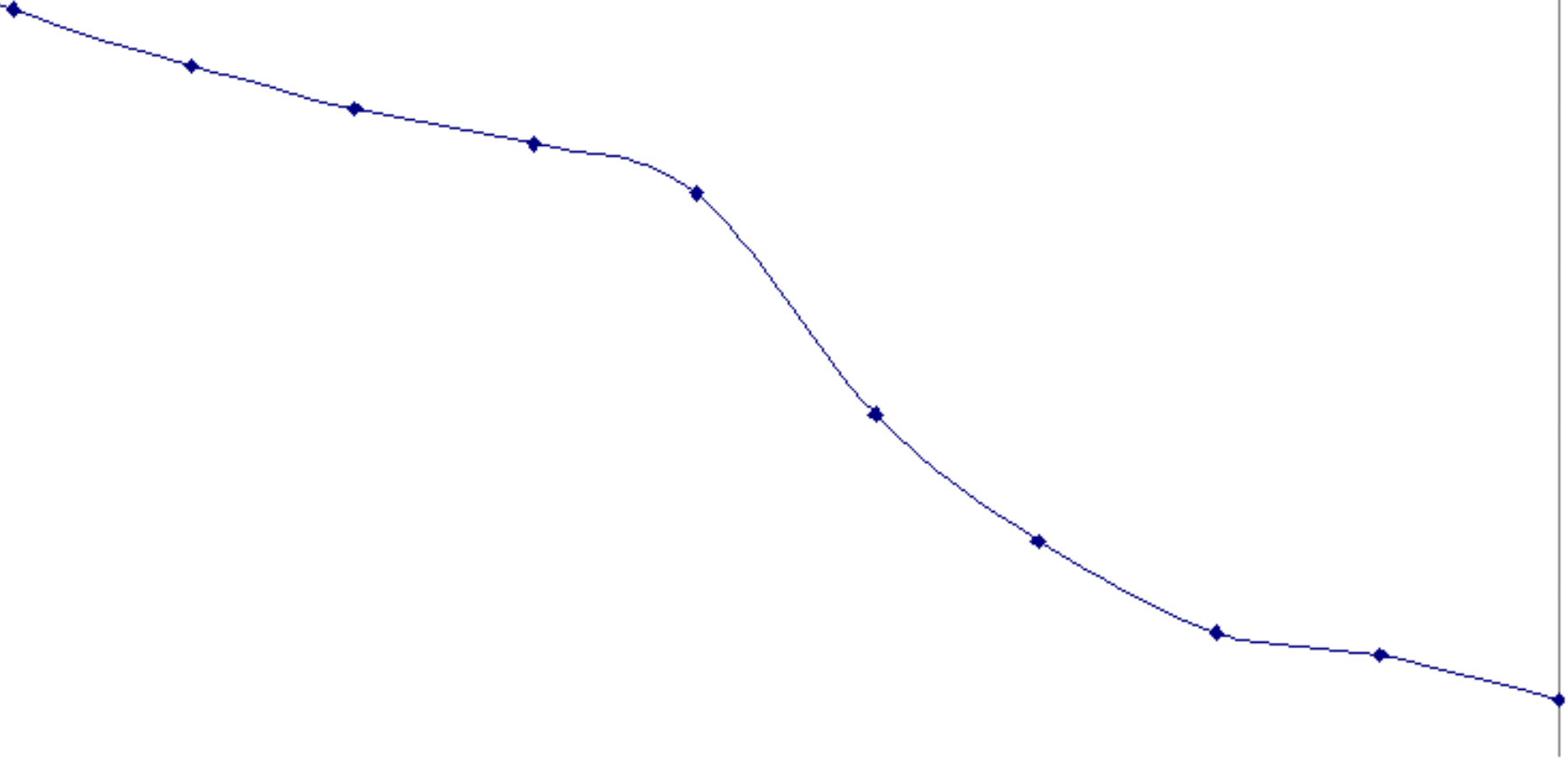
FY98 Annual Report

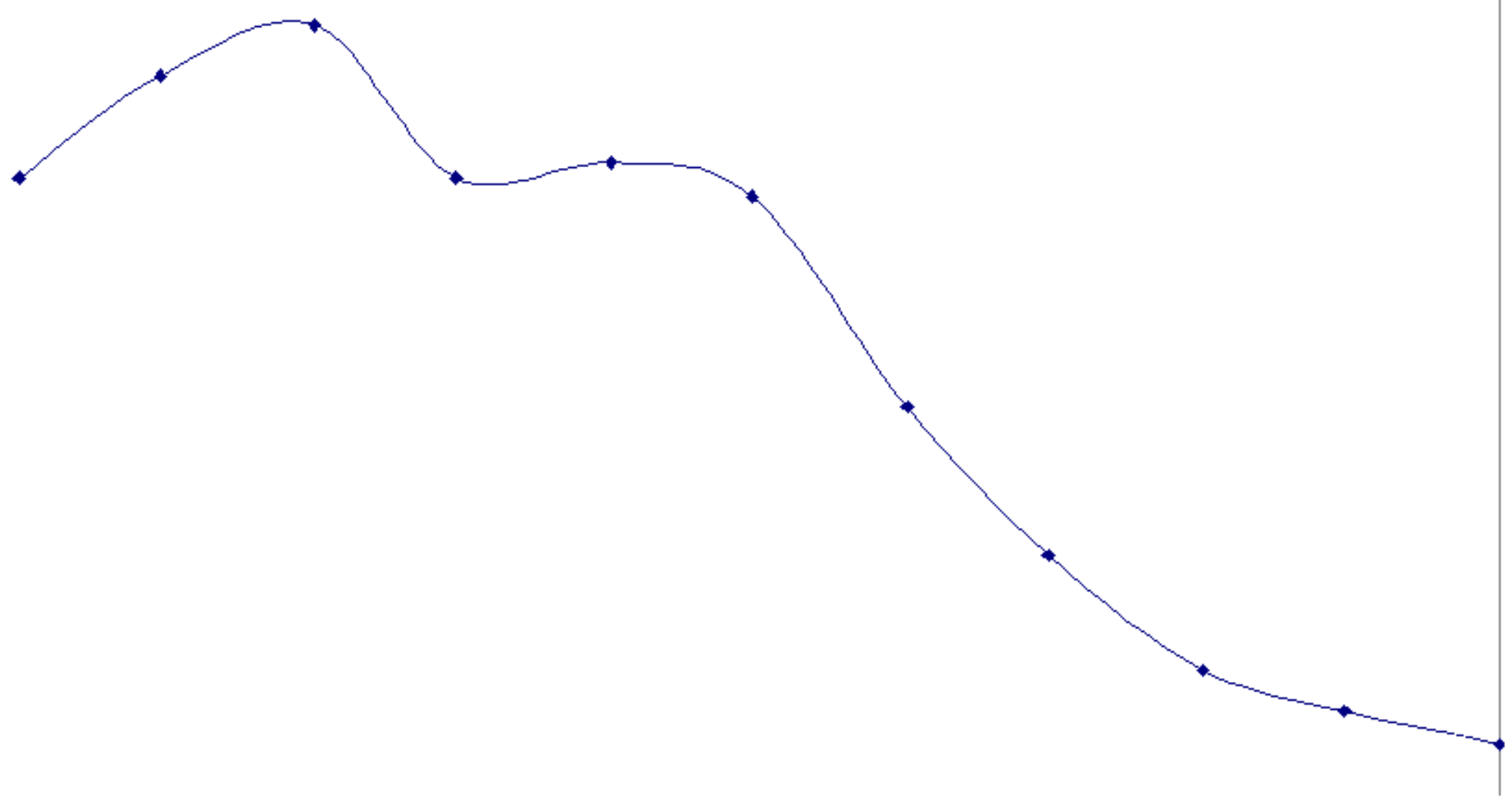

file://C|/ManualConversion/HTML/6792benk.htm (73 of 250)4/9/2004 4:05:24 AM 


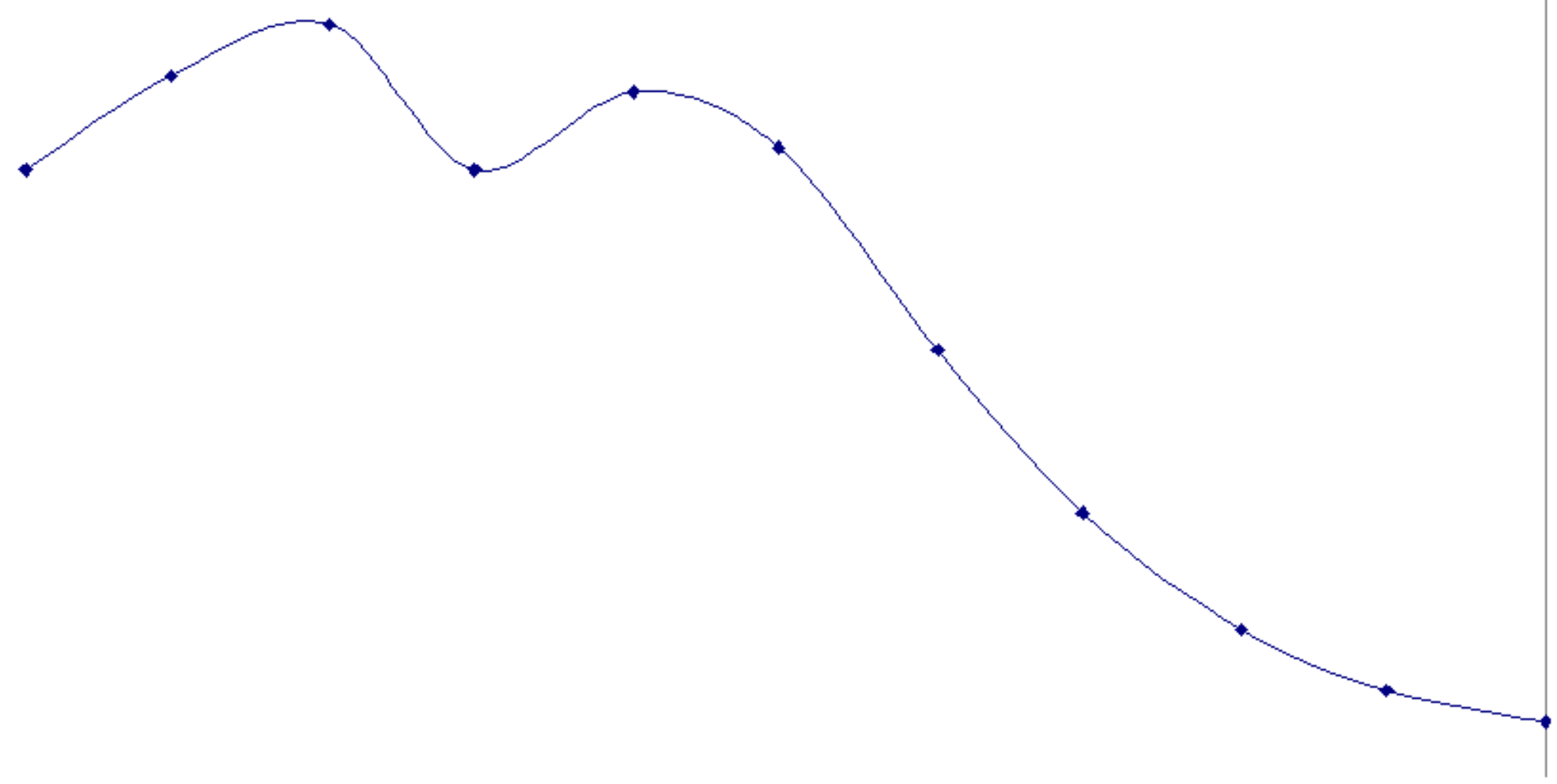


<smiles>CCCCC</smiles> 


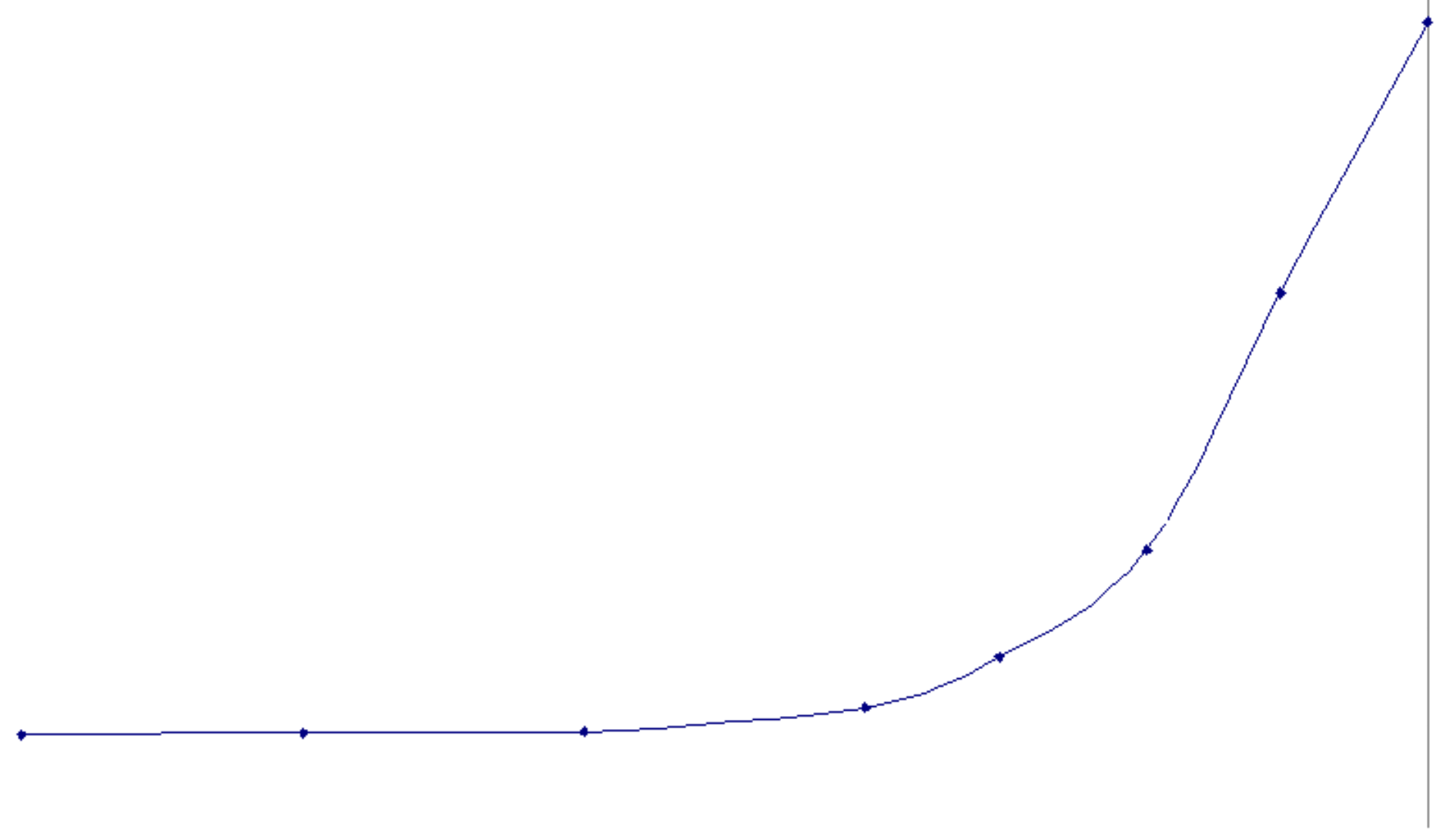



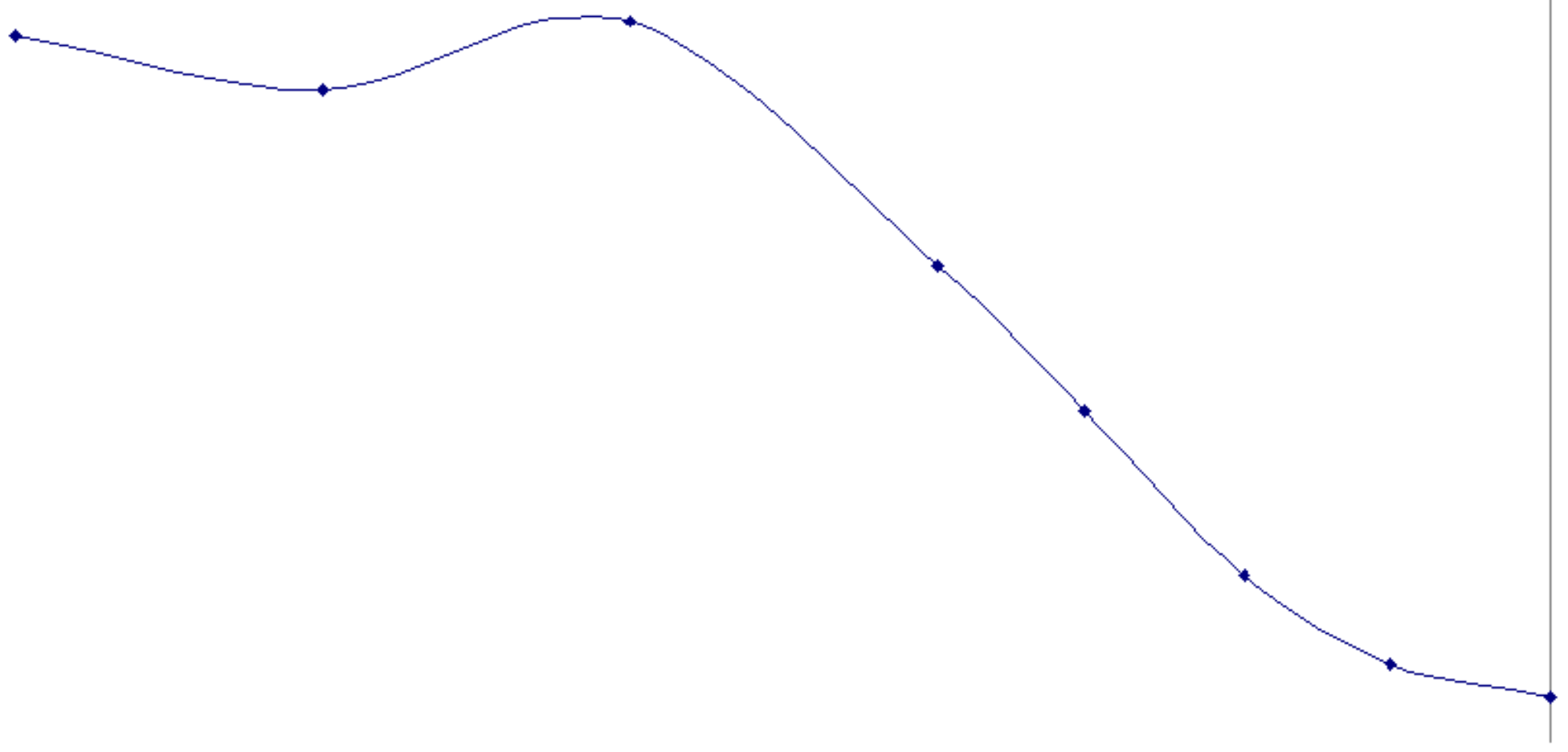
<smiles>CCCC</smiles> 
<smiles>CCCCCCCC</smiles> 


$$
7
$$




\section{Appendix G}

\section{Calibration Curves for Starrett M-1 Lubricant}

on Aluminum Panels and Stainless Steel Disks

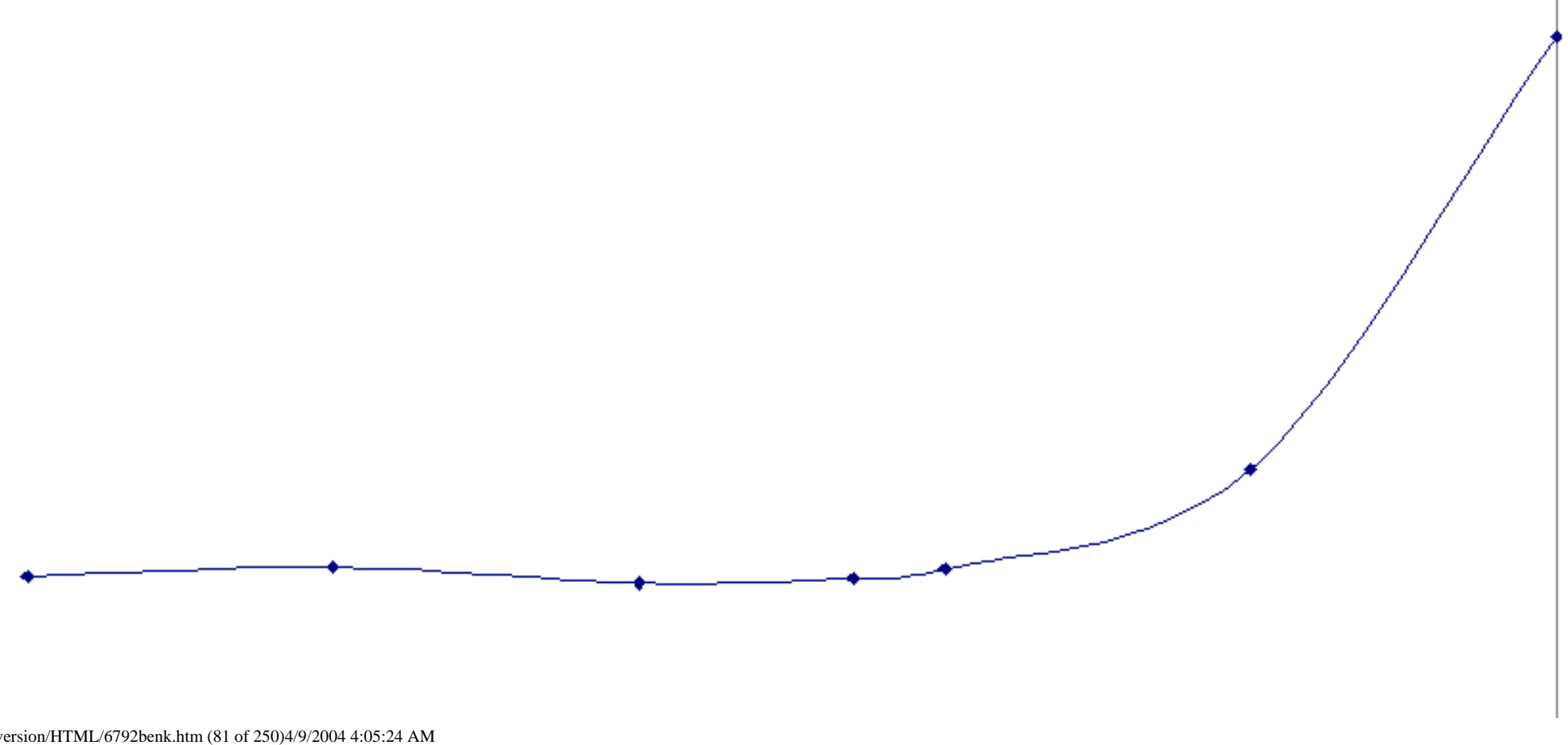


<smiles>C=CCC</smiles> 

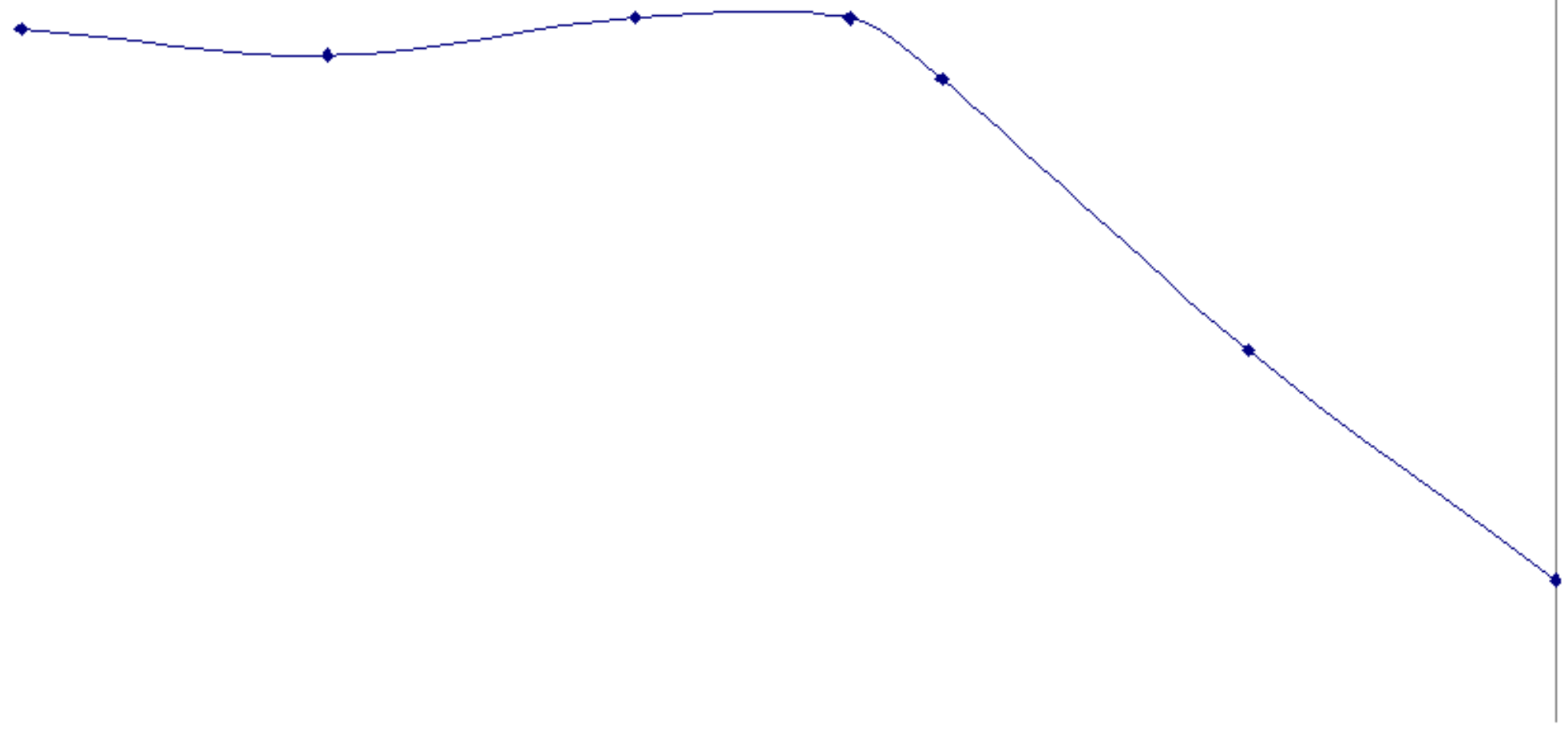
$+$

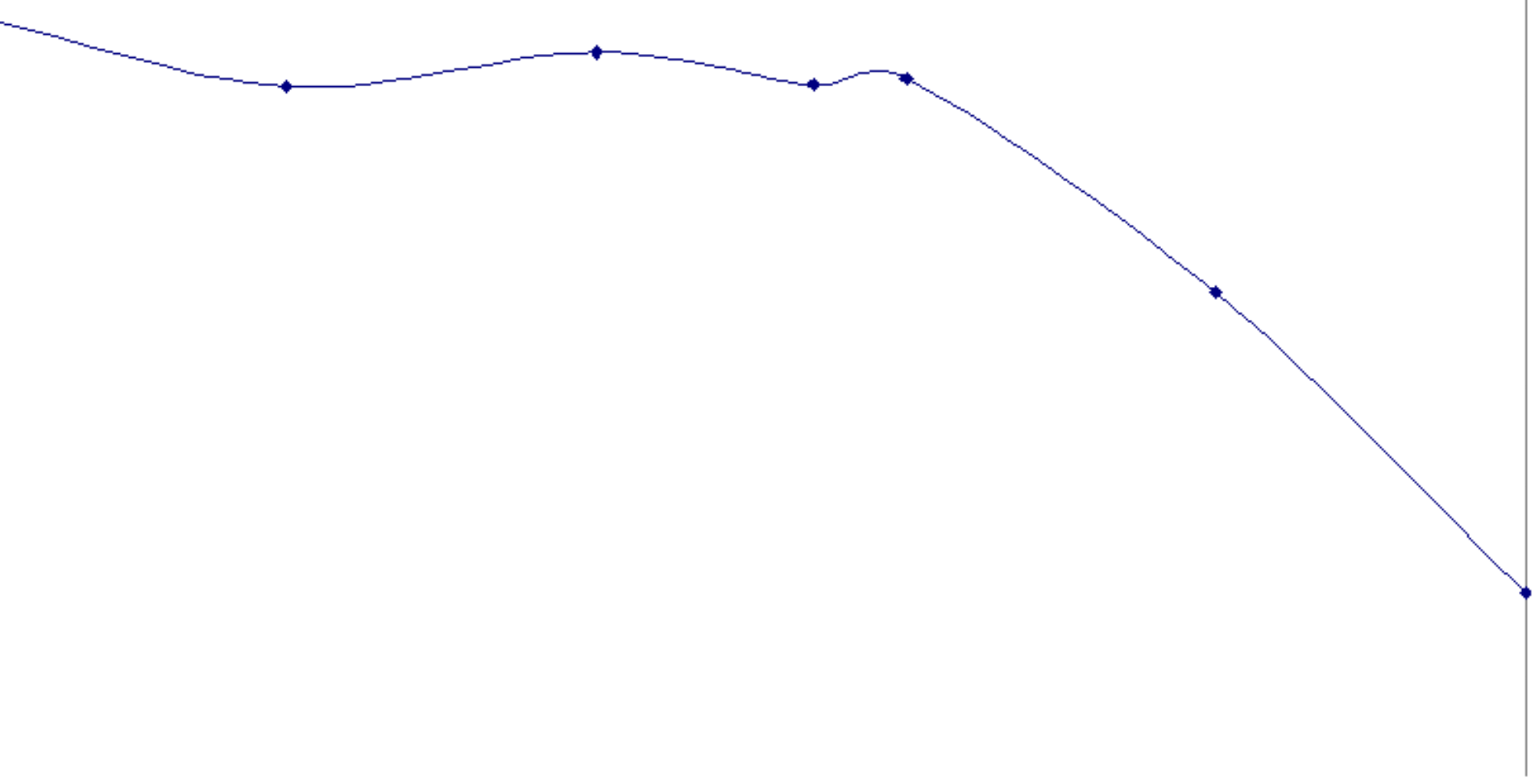




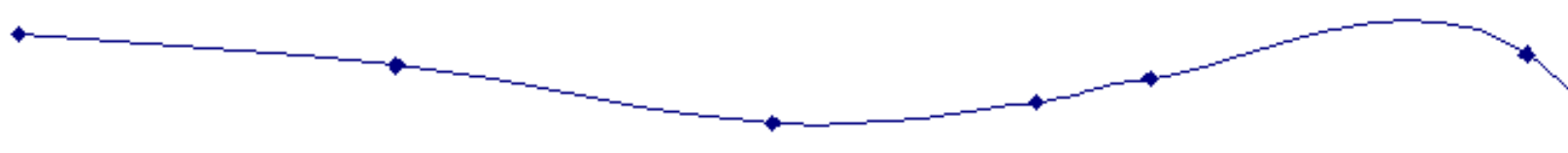




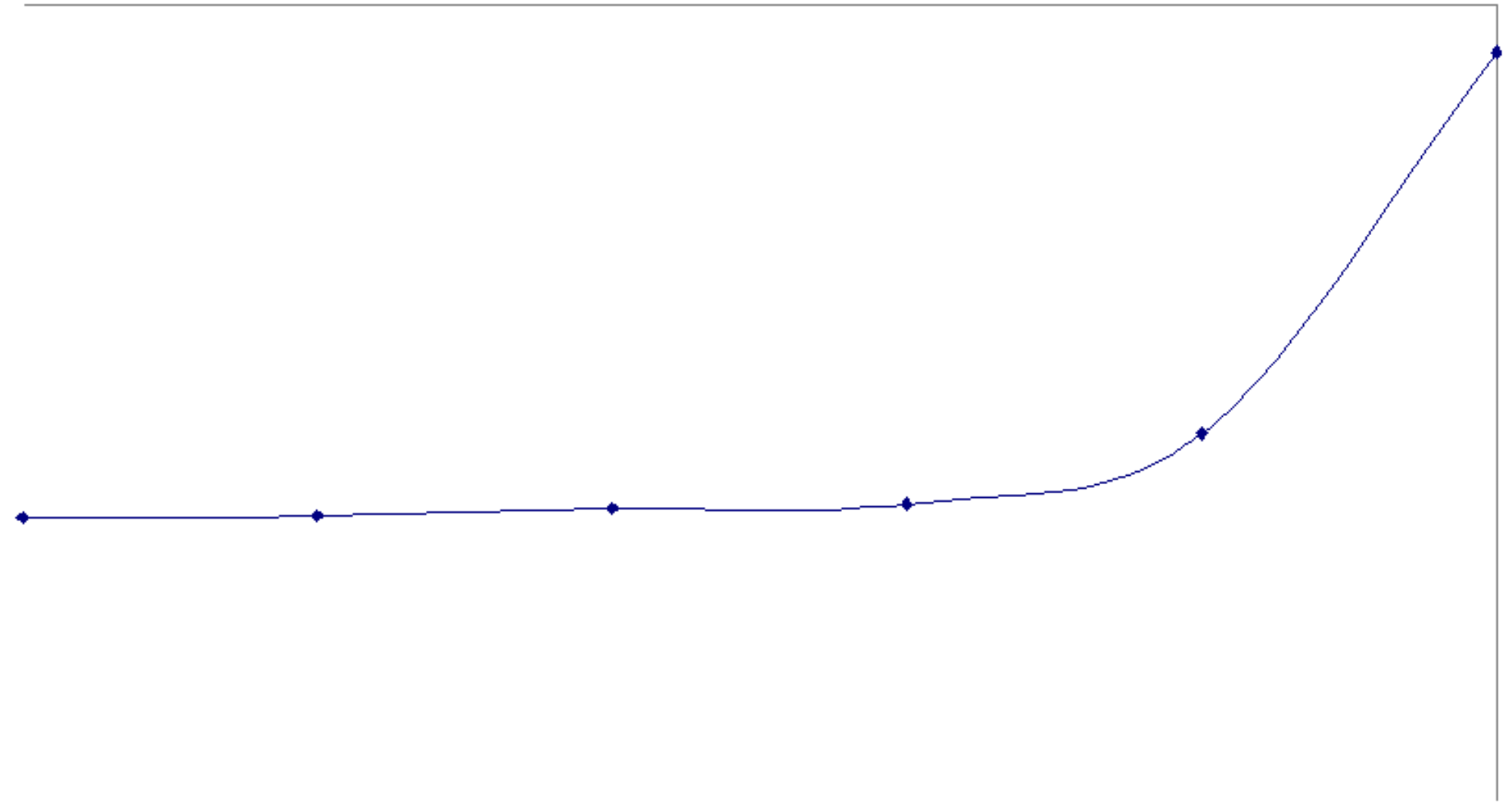



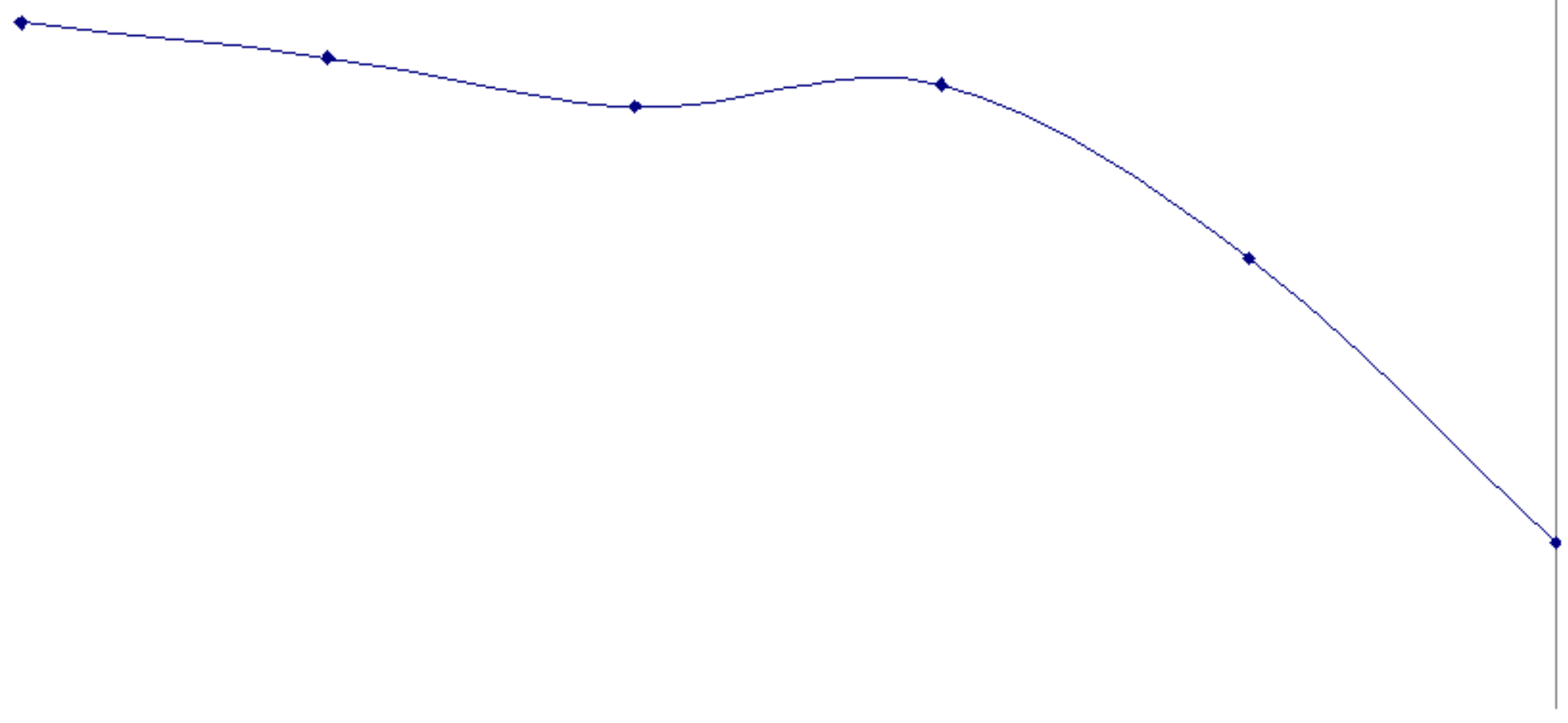


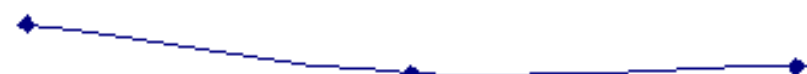




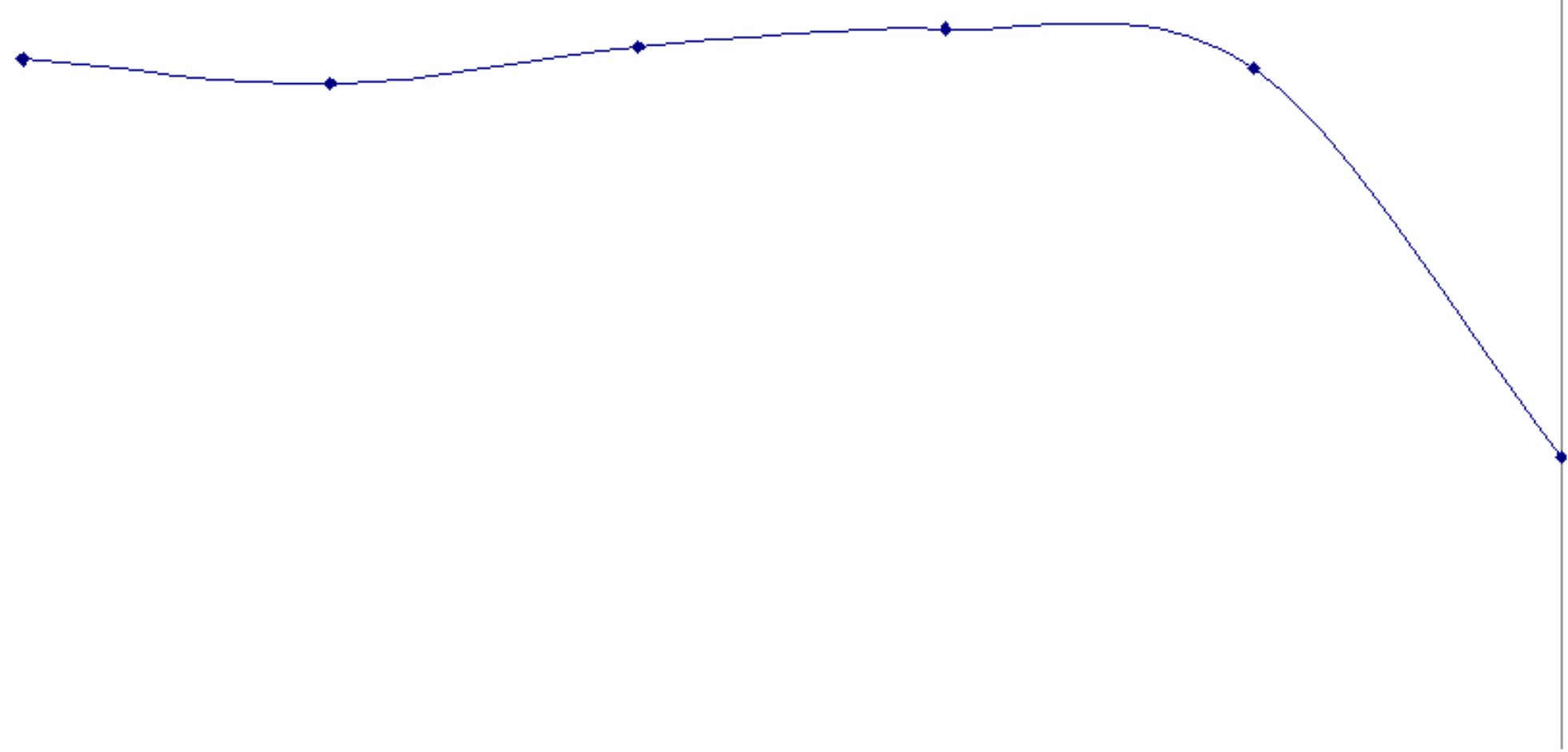




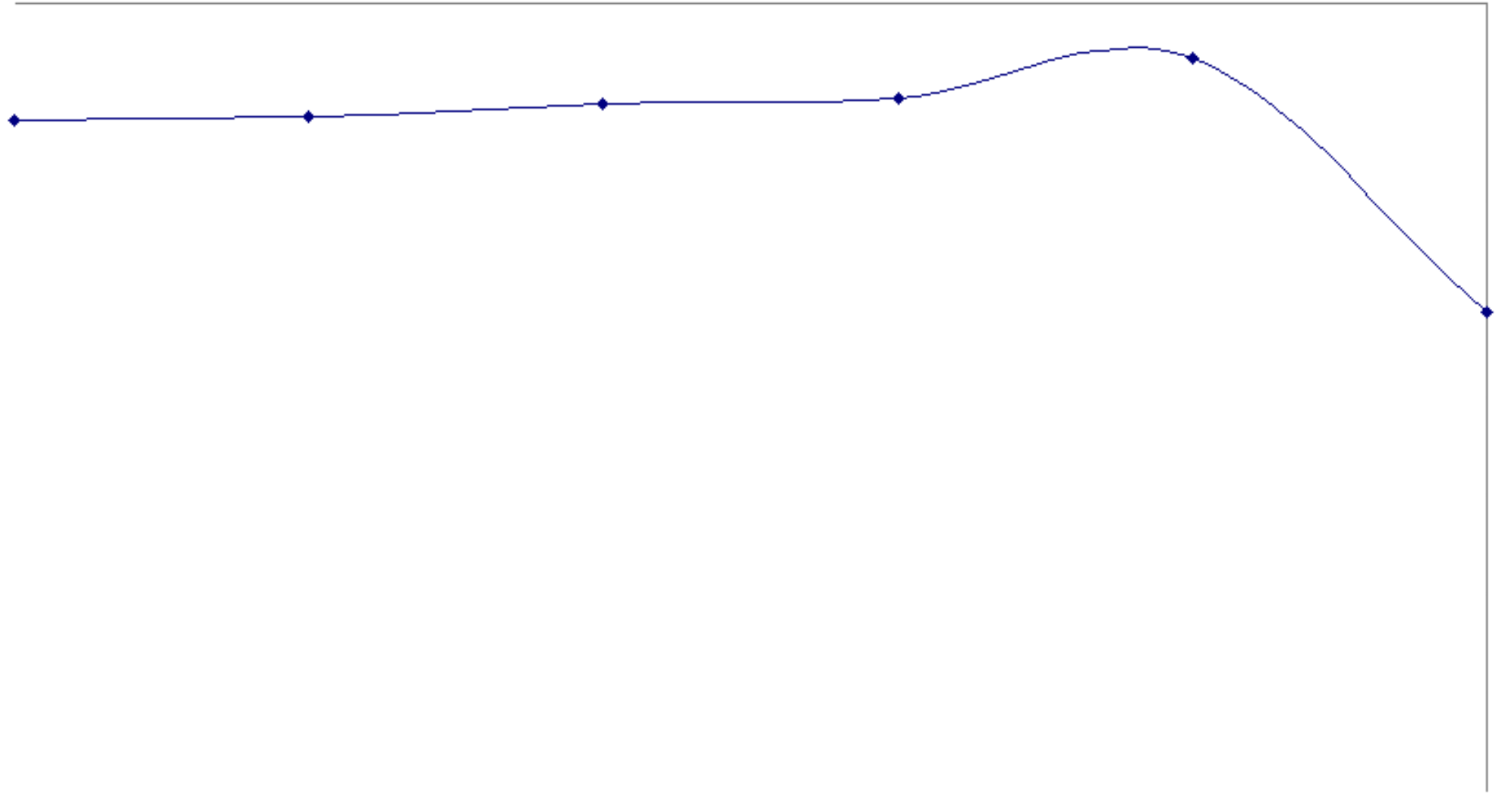




\section{Appendix H}

\section{Calibration Curves for Vaseline}

on Aluminum Panels and Various Stainless Steel Disks

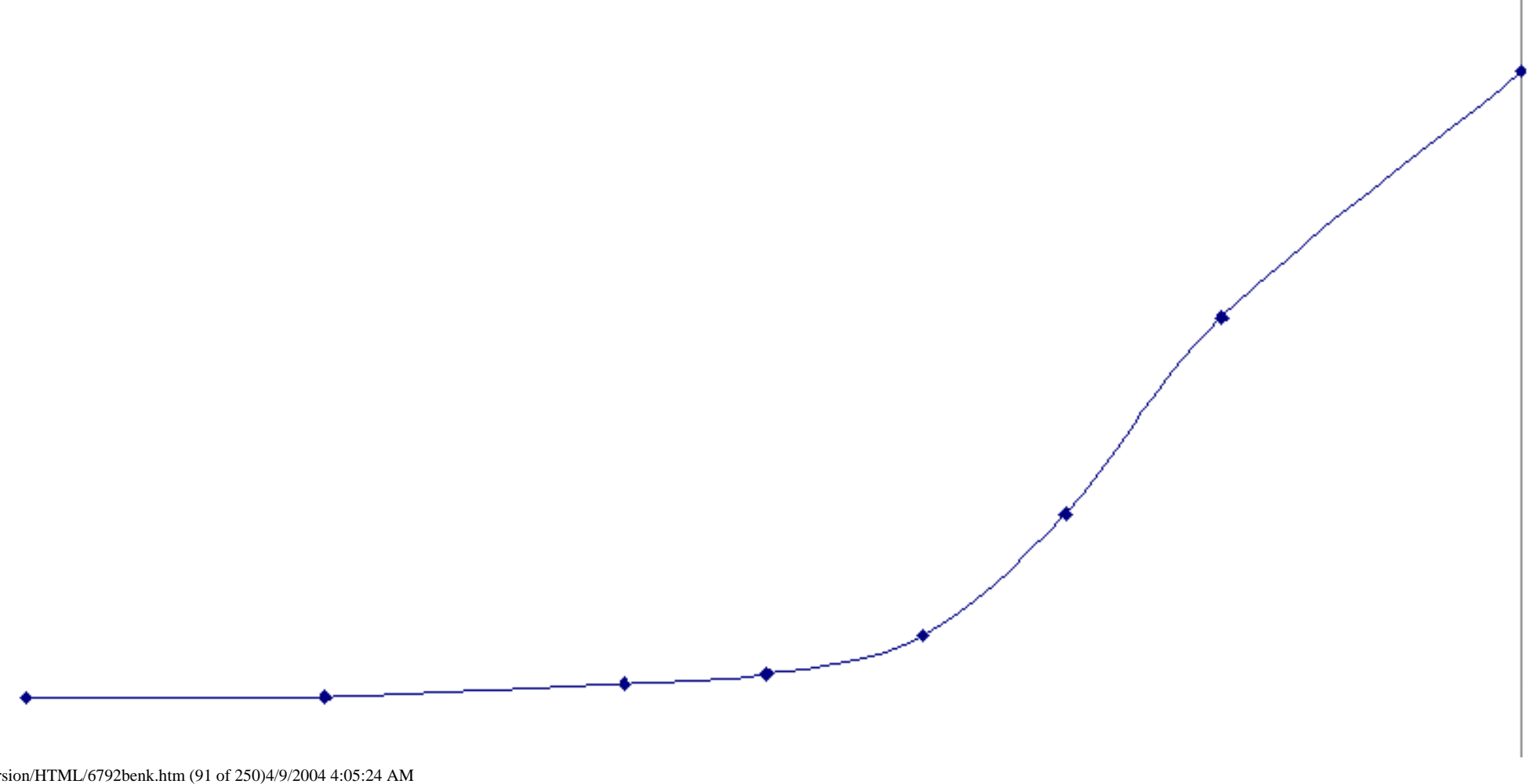


<smiles>CCC</smiles> 


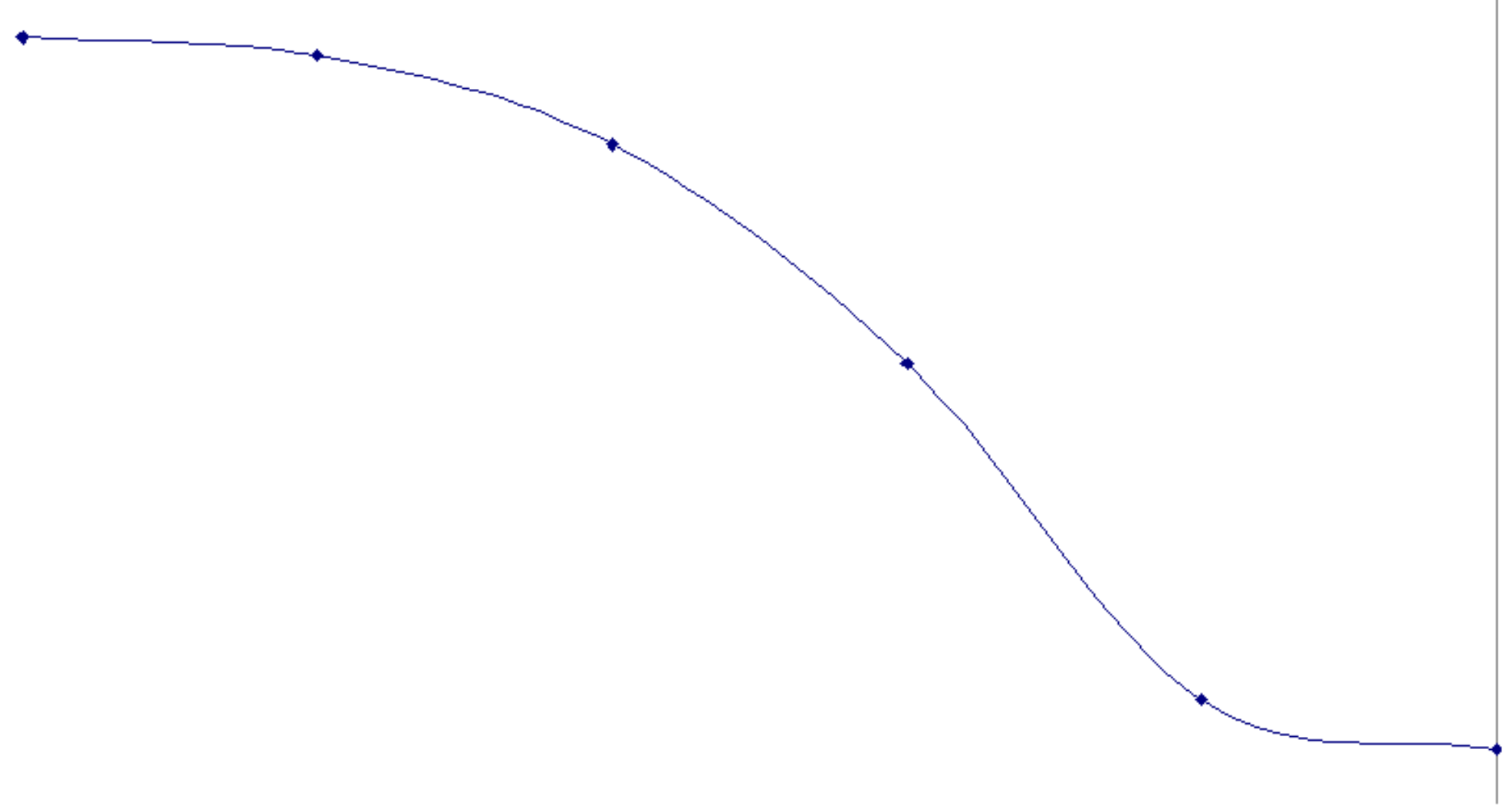


FY98 Annual Report

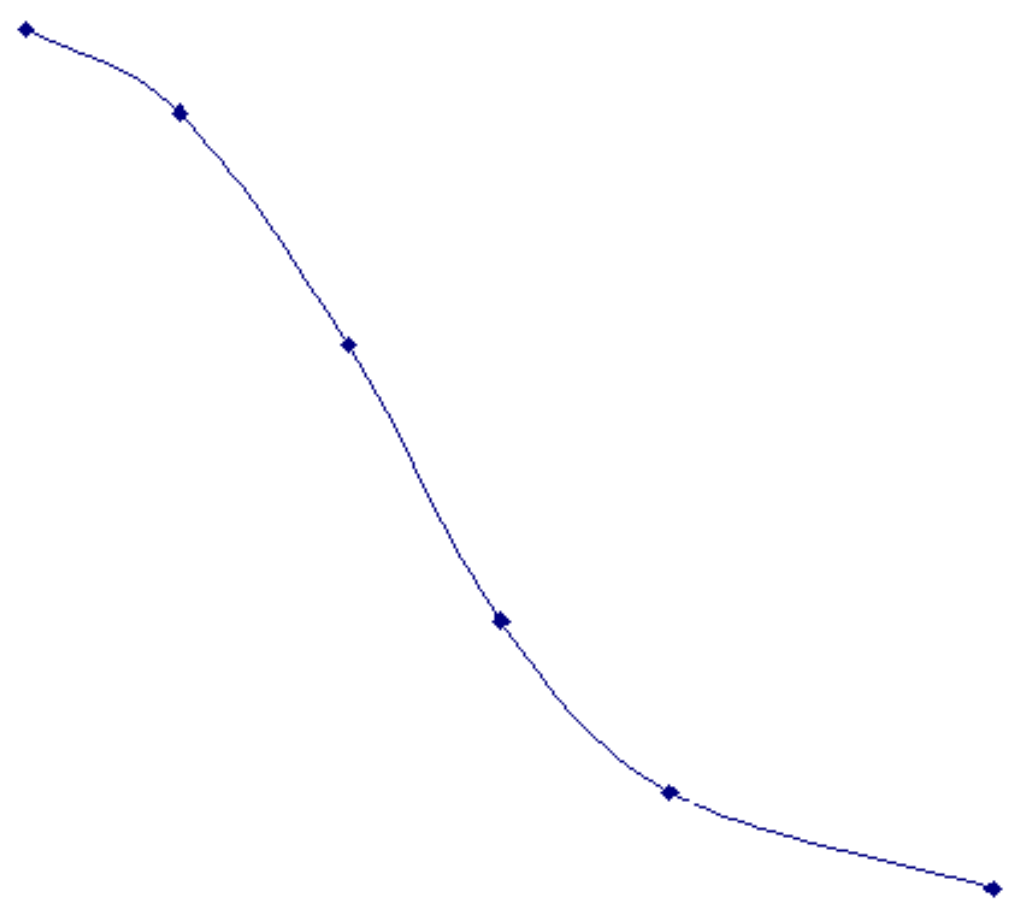

file://C//ManualConversion/HTML/6792benk.htm (94 of 250)4/9/2004 4:05:24 AM 


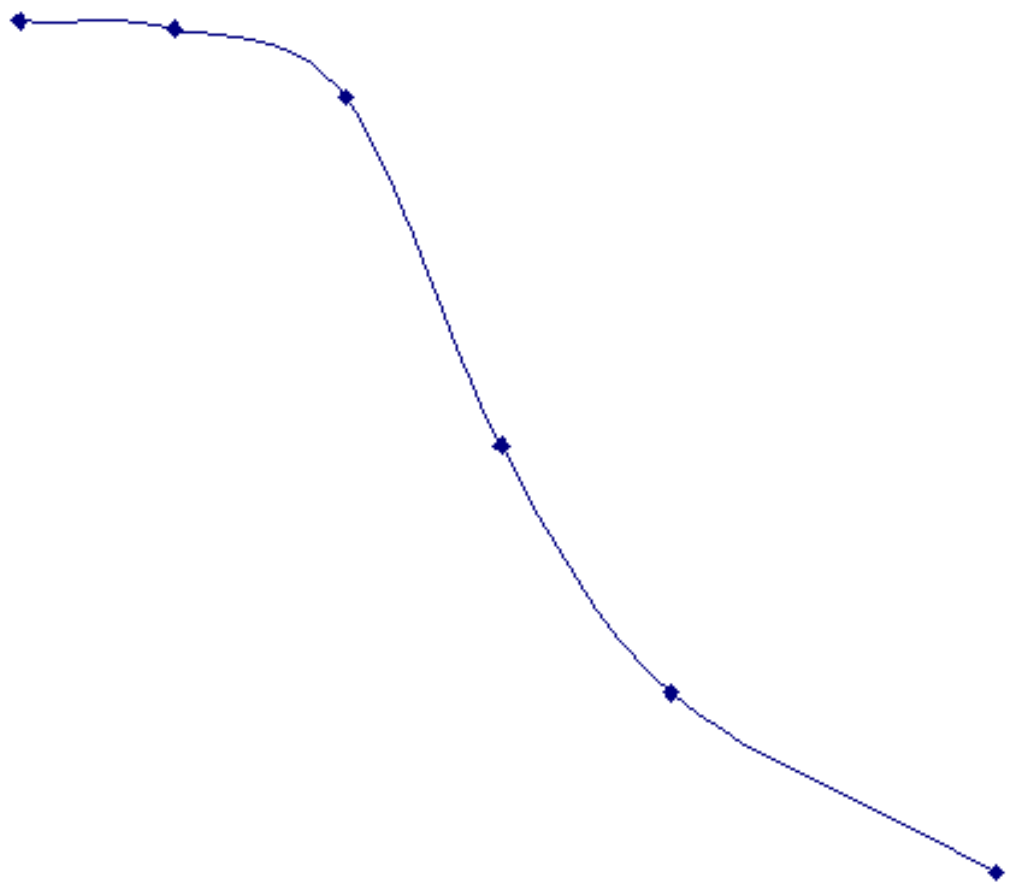

file:///C/ManualConversion/HTML/6792benk.htm (95 of 250)4/9/2004 4:05:24 AM 


$$
Y
$$


FY98 Annual Report

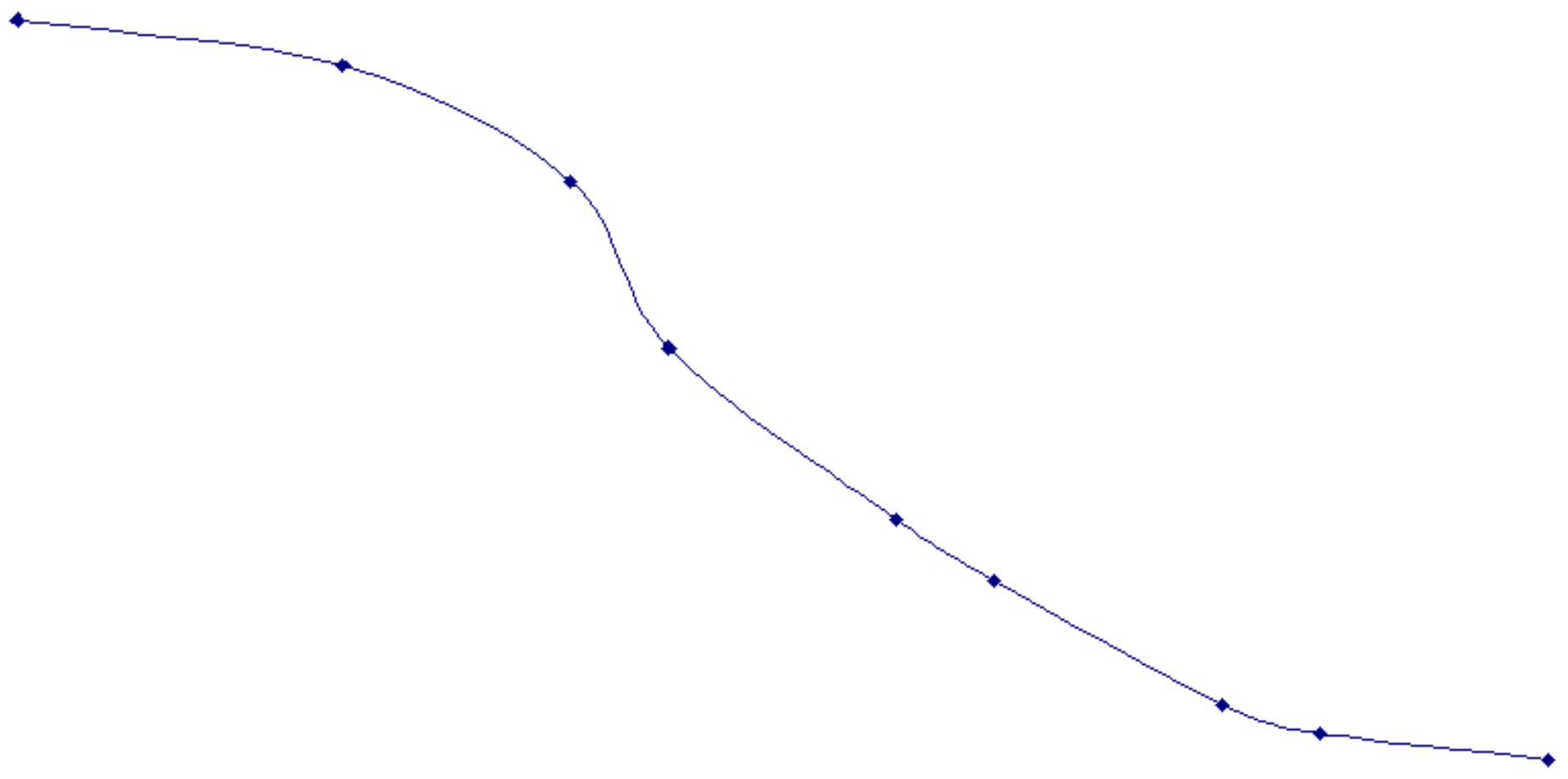

file:///C/ManualConversion/HTML/6792benk.htm (97 of 250)4/9/2004 4:05:24 AM 
<smiles>C1CCC1</smiles> 
<smiles>CCCCCCCC</smiles> 


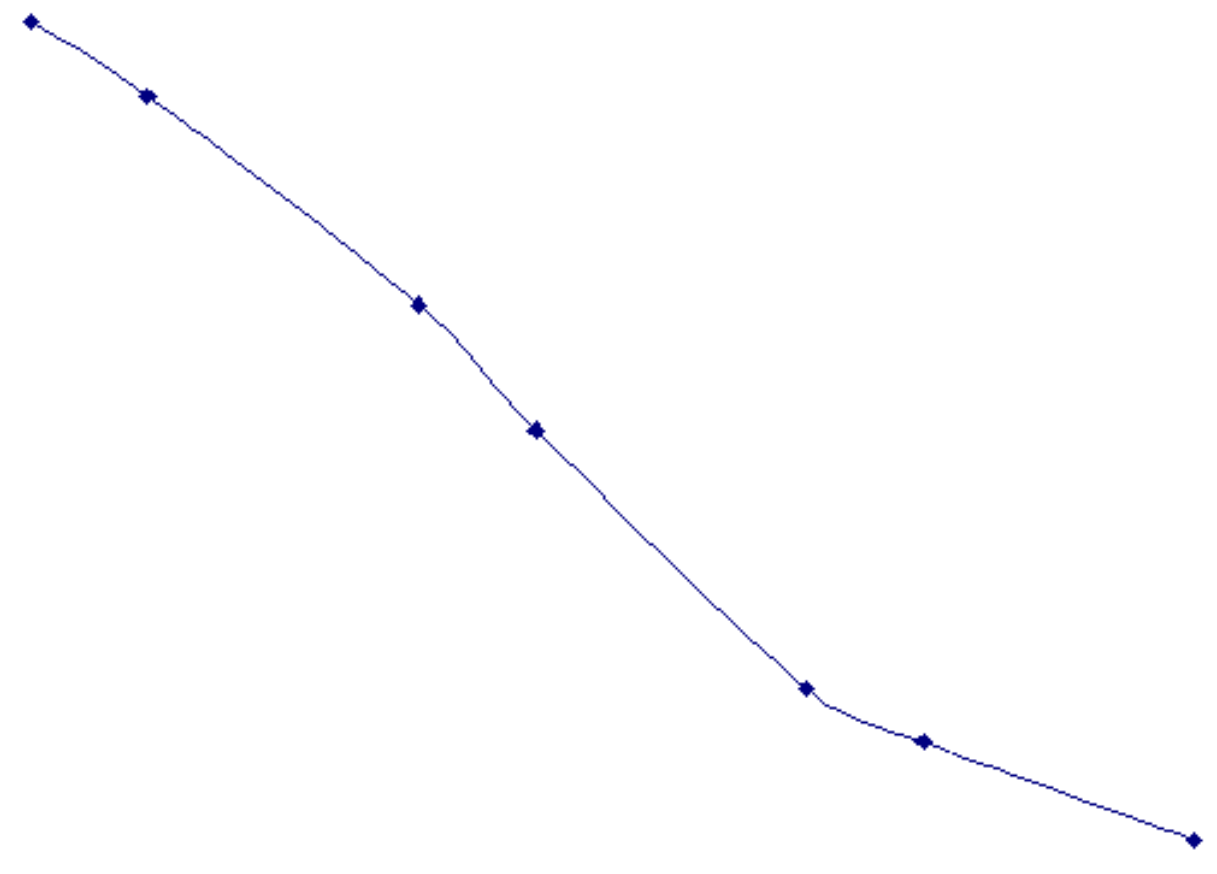


<smiles>CCCC</smiles> 
FY98 Annual Report

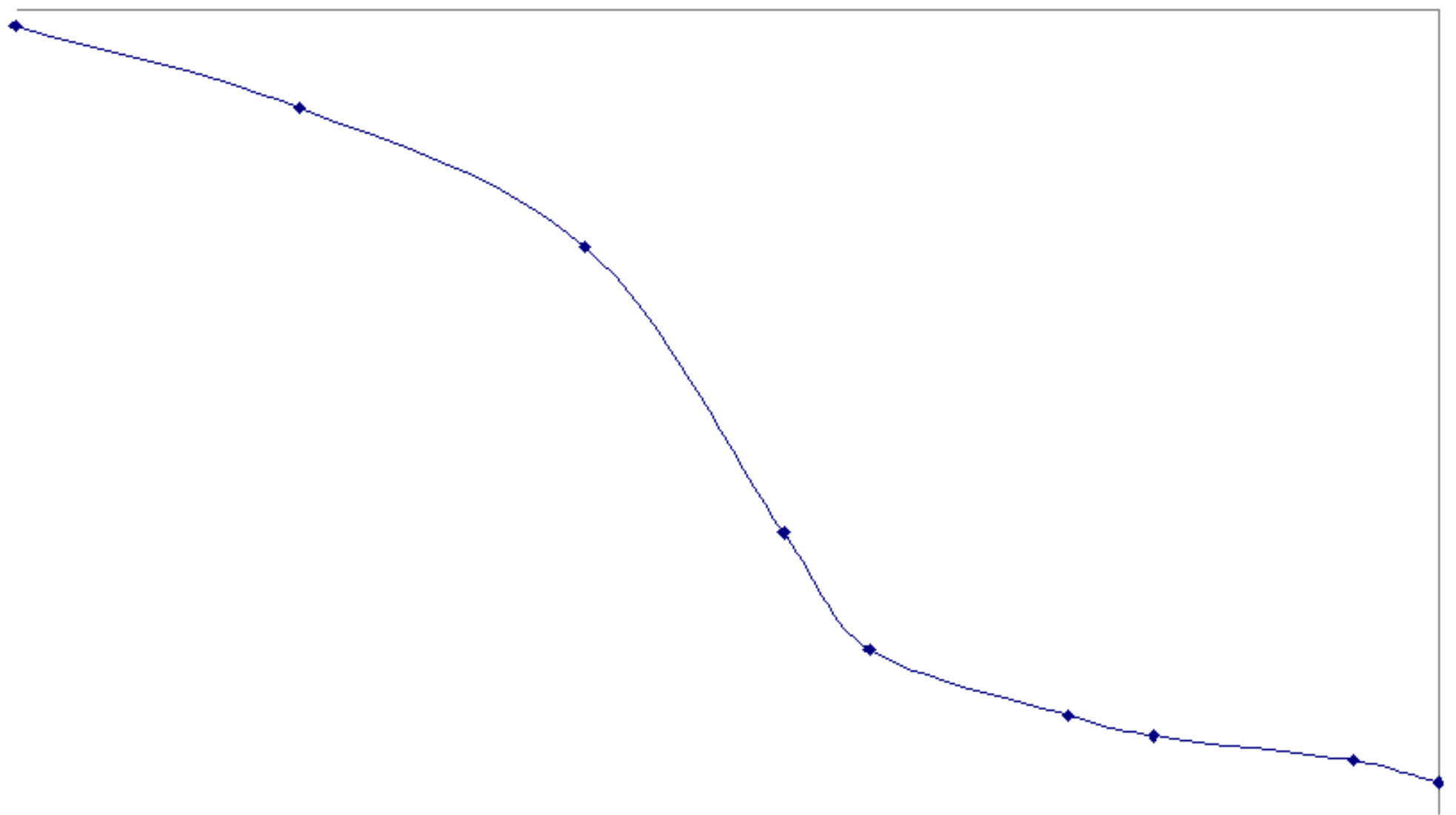

file://C//ManualConversion/HTML/6792benk.htm (102 of 250)4/9/2004 4:05:24 AM 


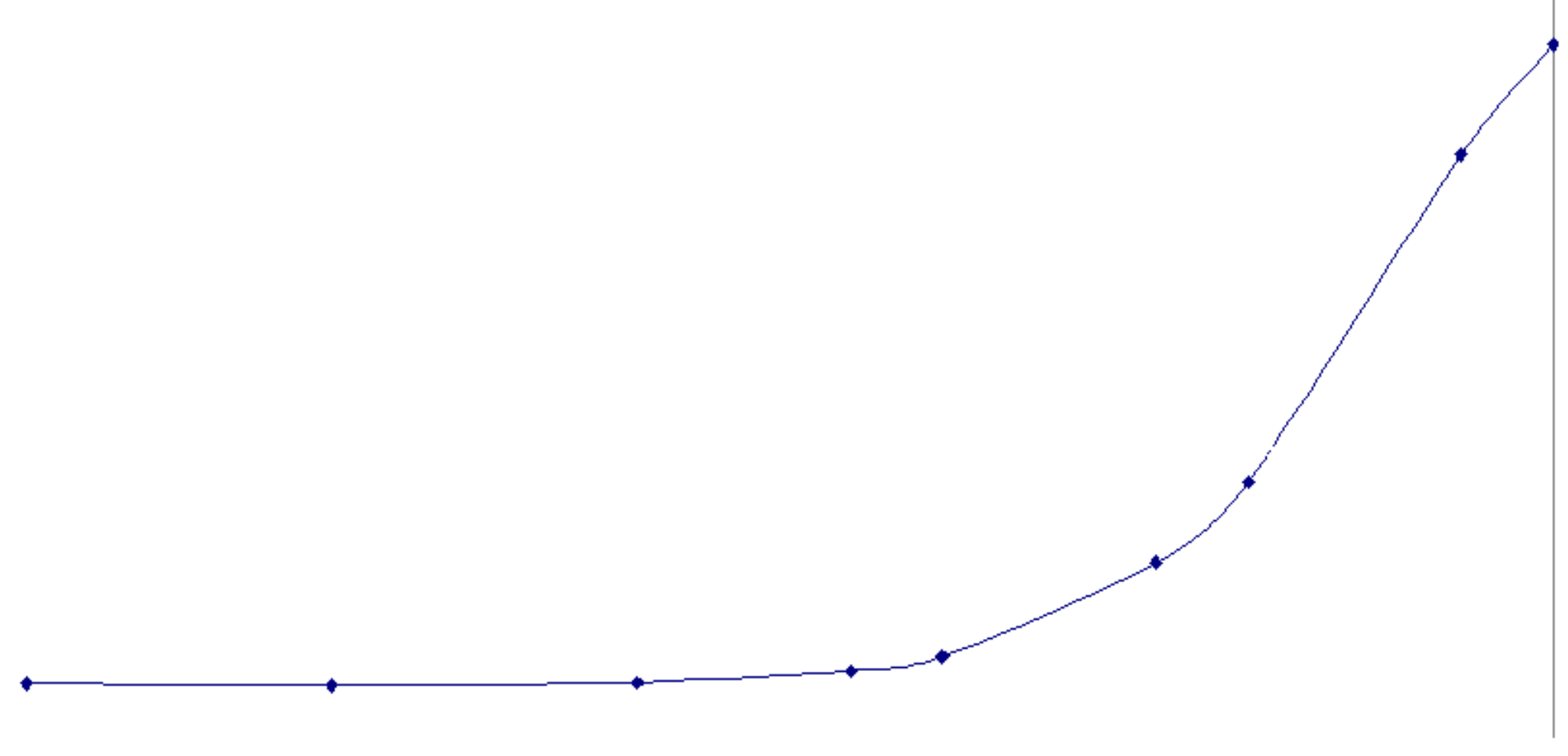




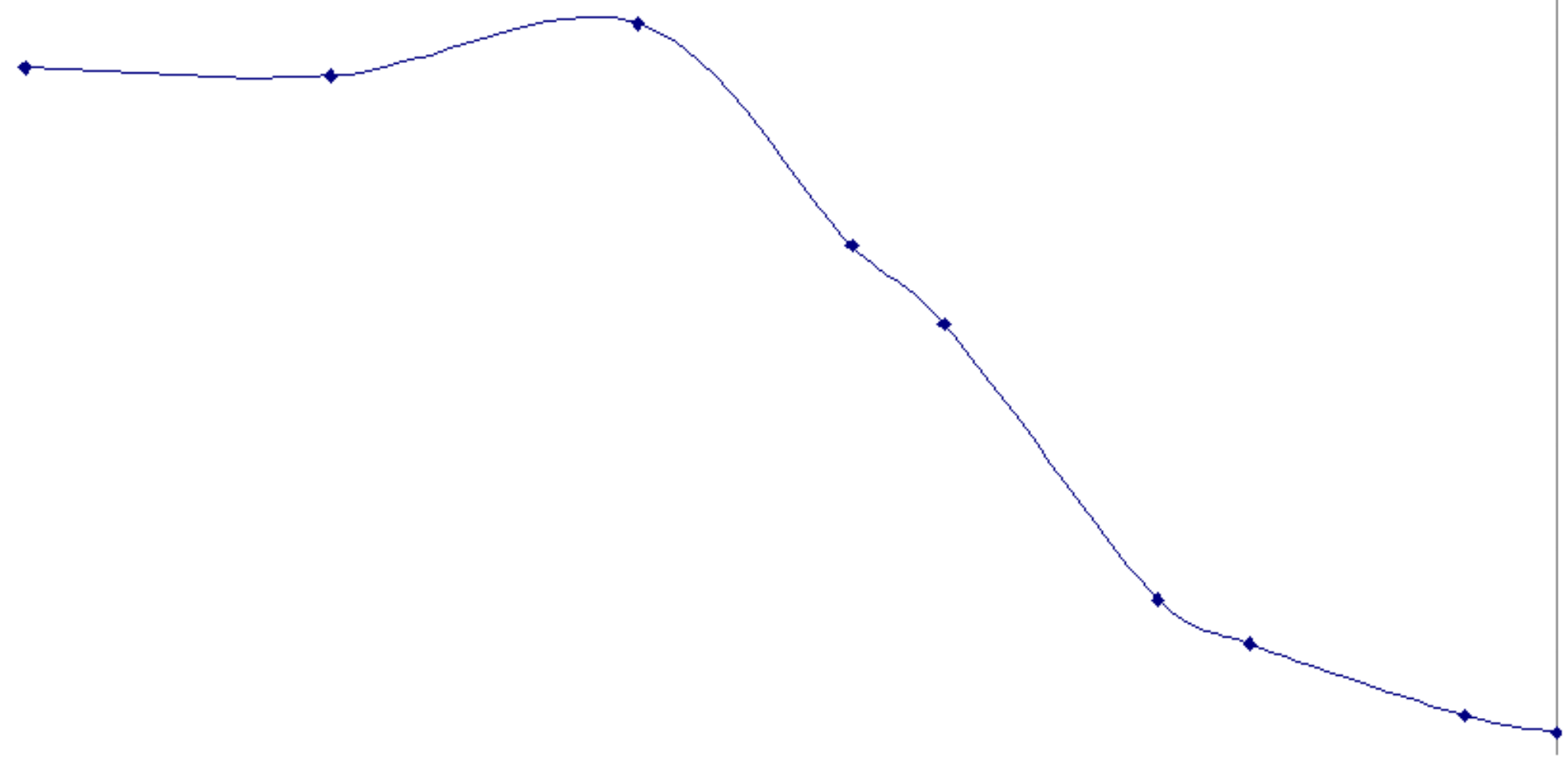




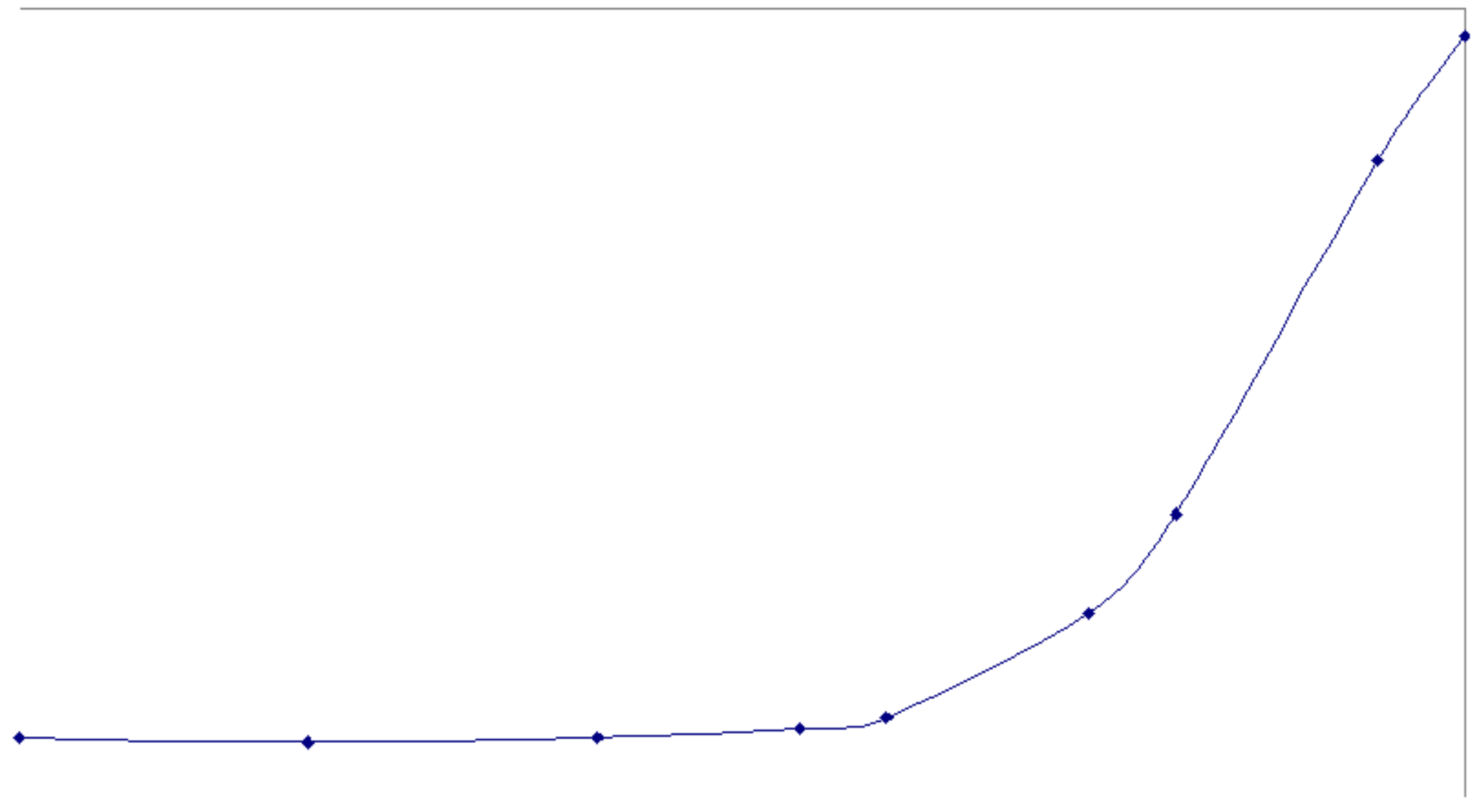



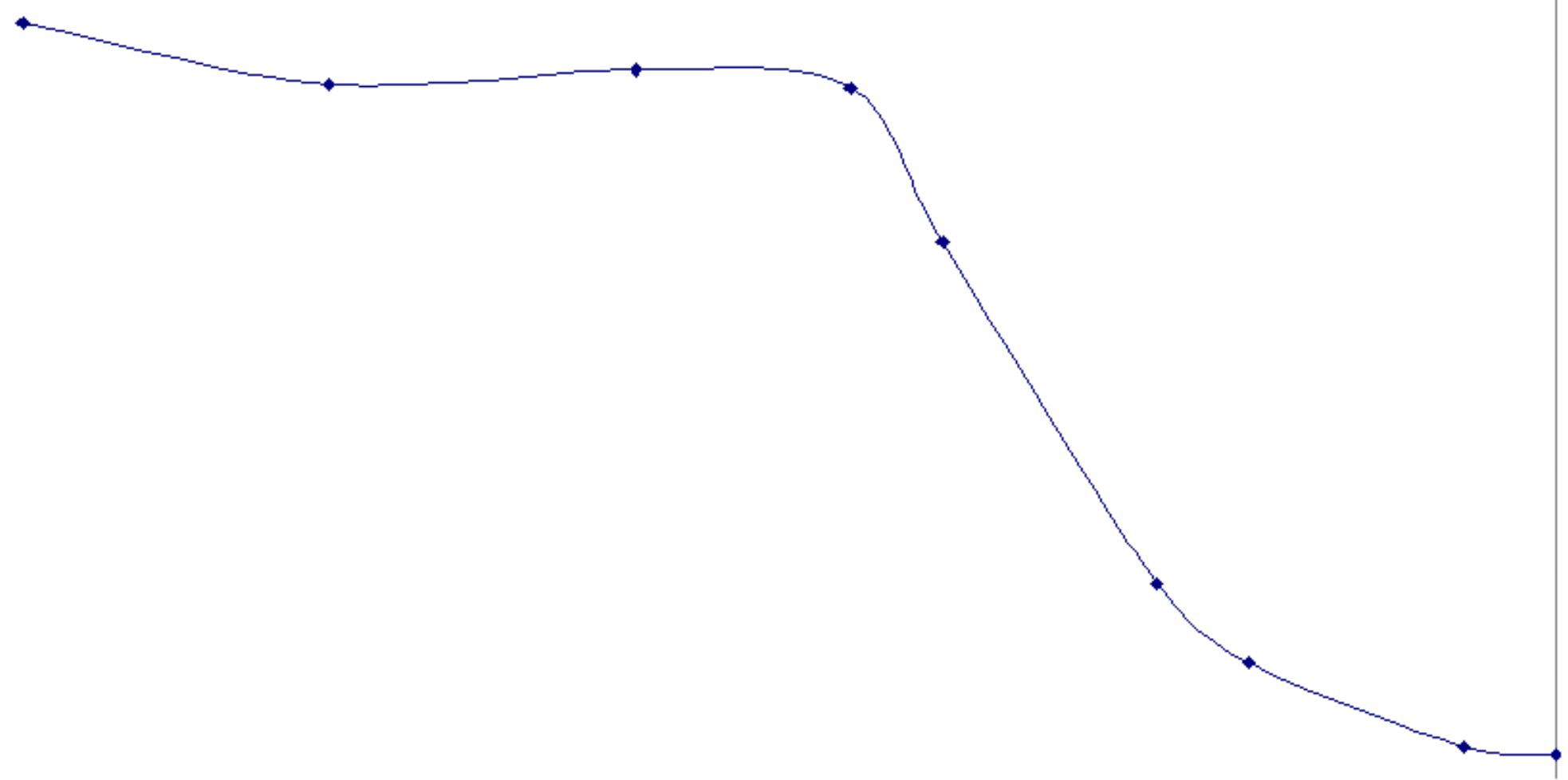


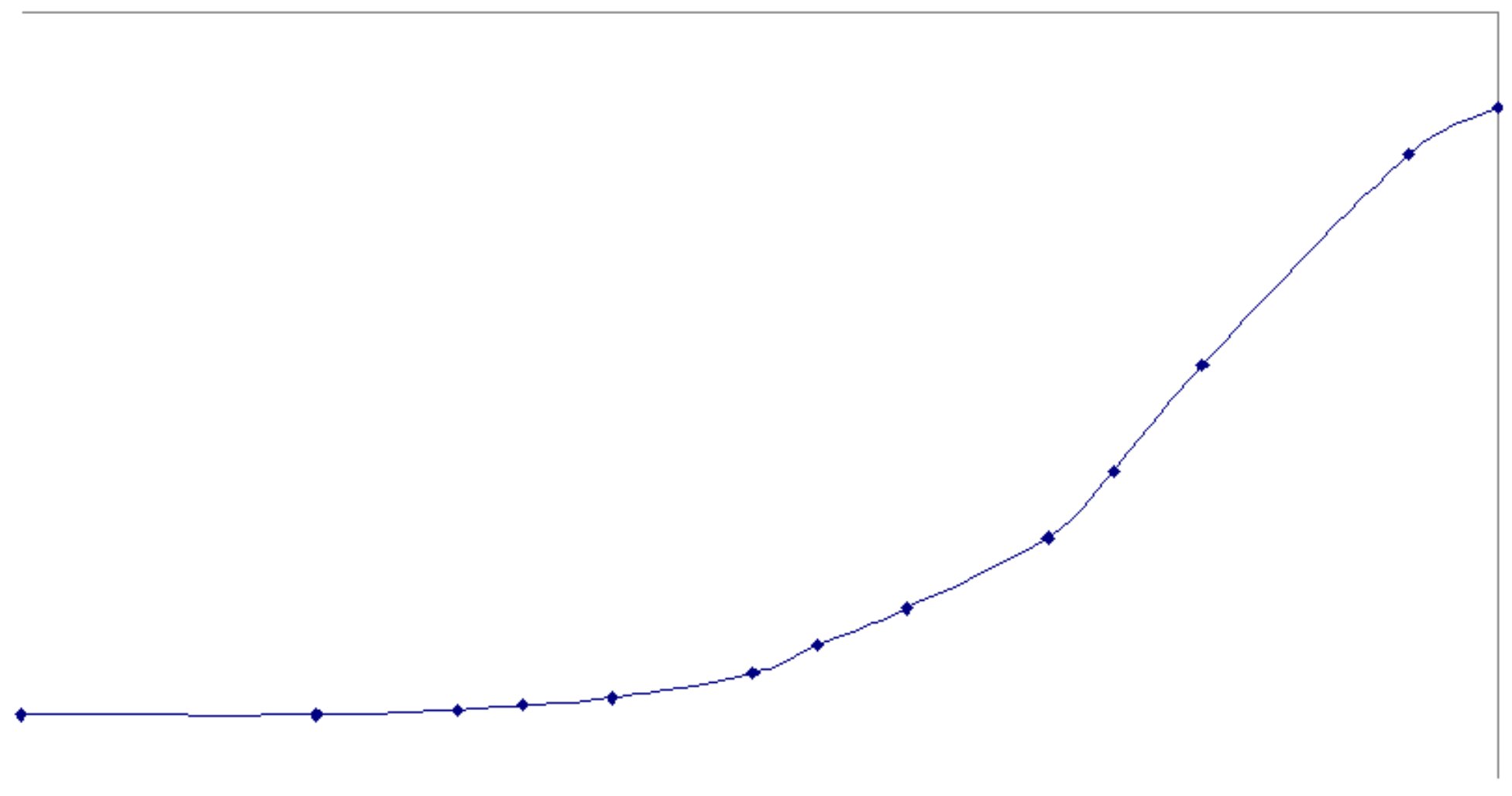


FY98 Annual Report

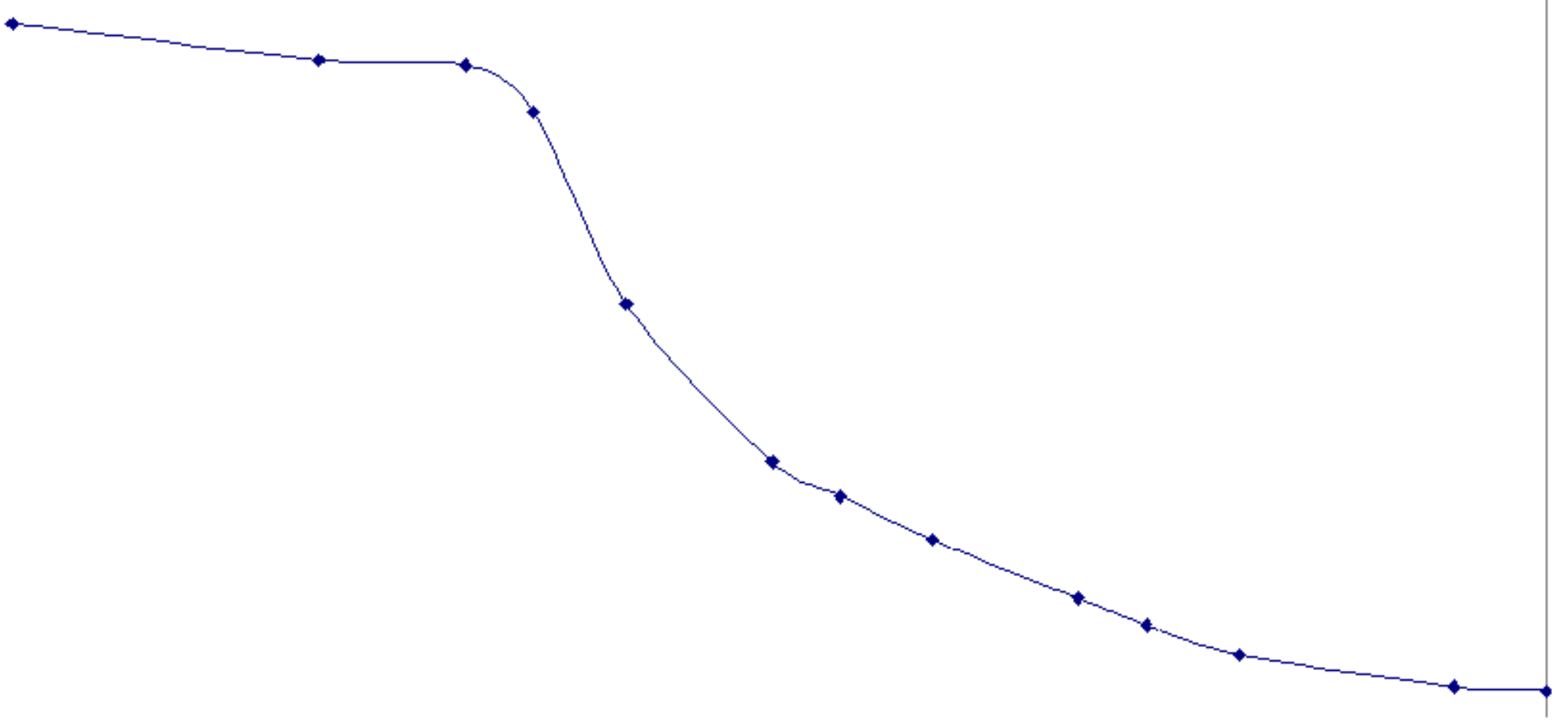

file://C//ManualConversion/HTML/6792benk.htm (108 of 250)4/9/2004 4:05:24 AM 
FY98 Annual Report

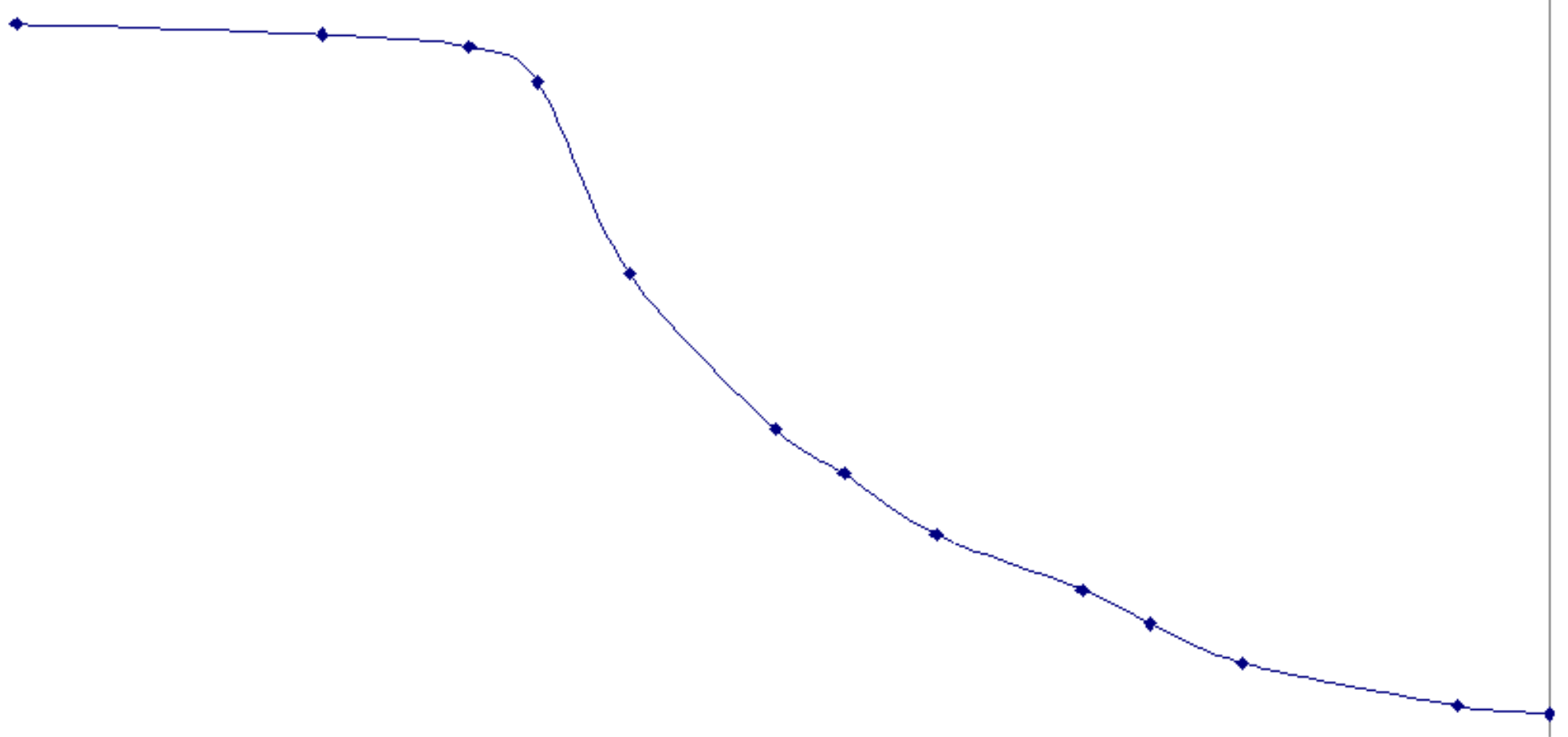

file:///C//ManualConversion/HTML/6792benk.htm (109 of 250)4/9/2004 4:05:24 AM 
FY98 Annual Report

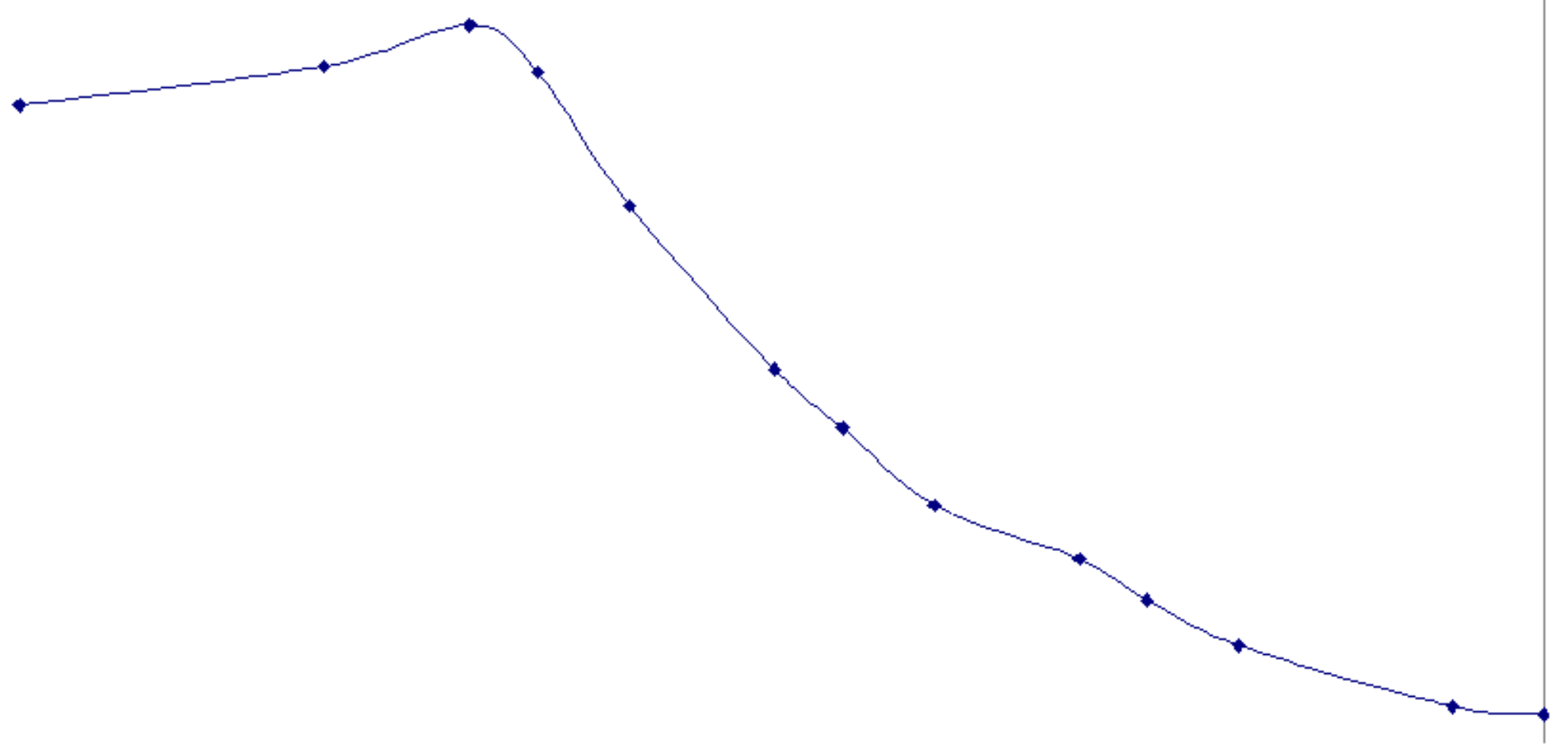

file:///C|/ManualConversion/HTML/6792benk.htm (110 of 250)4/9/2004 4:05:24 AM 

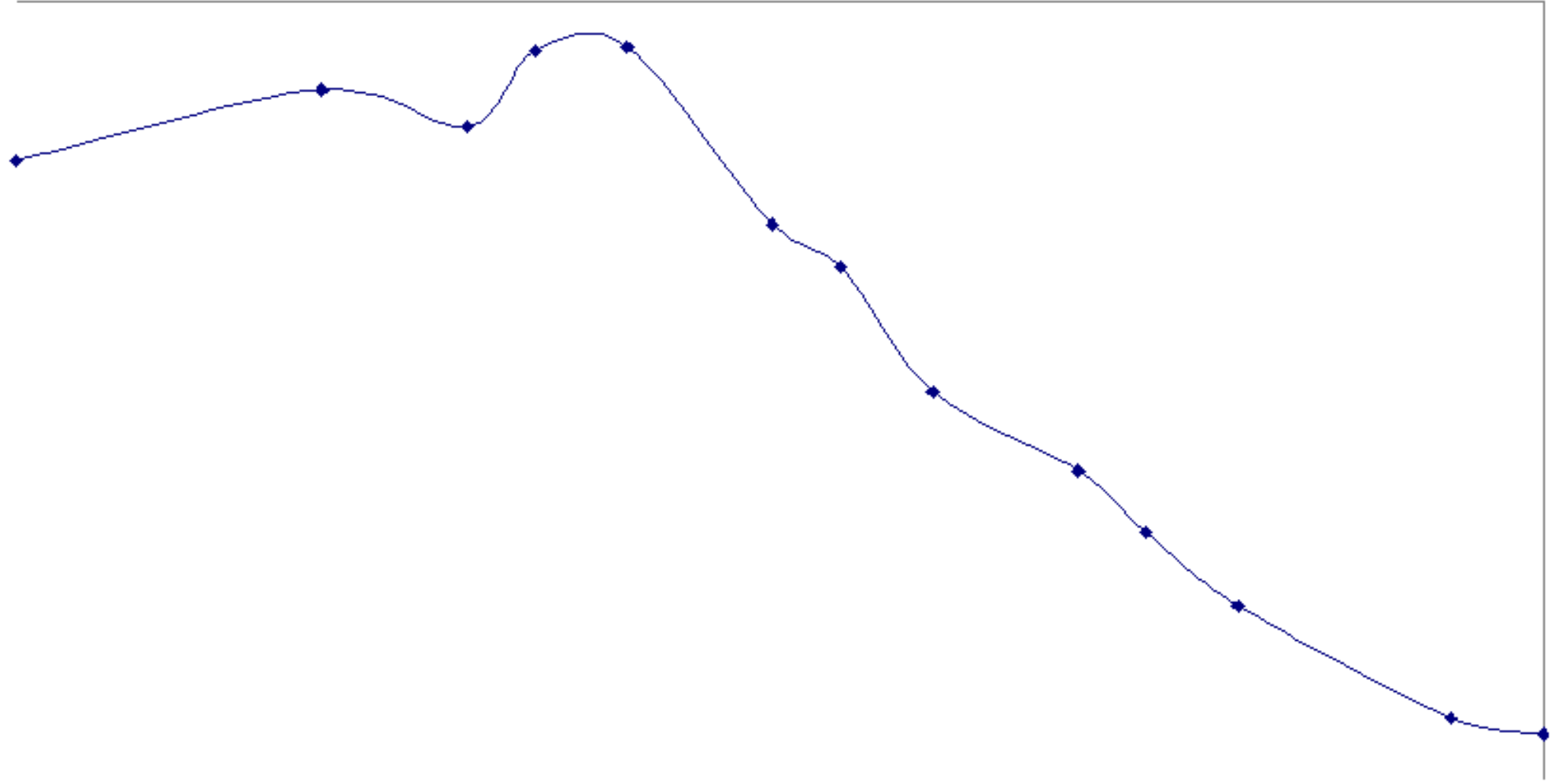


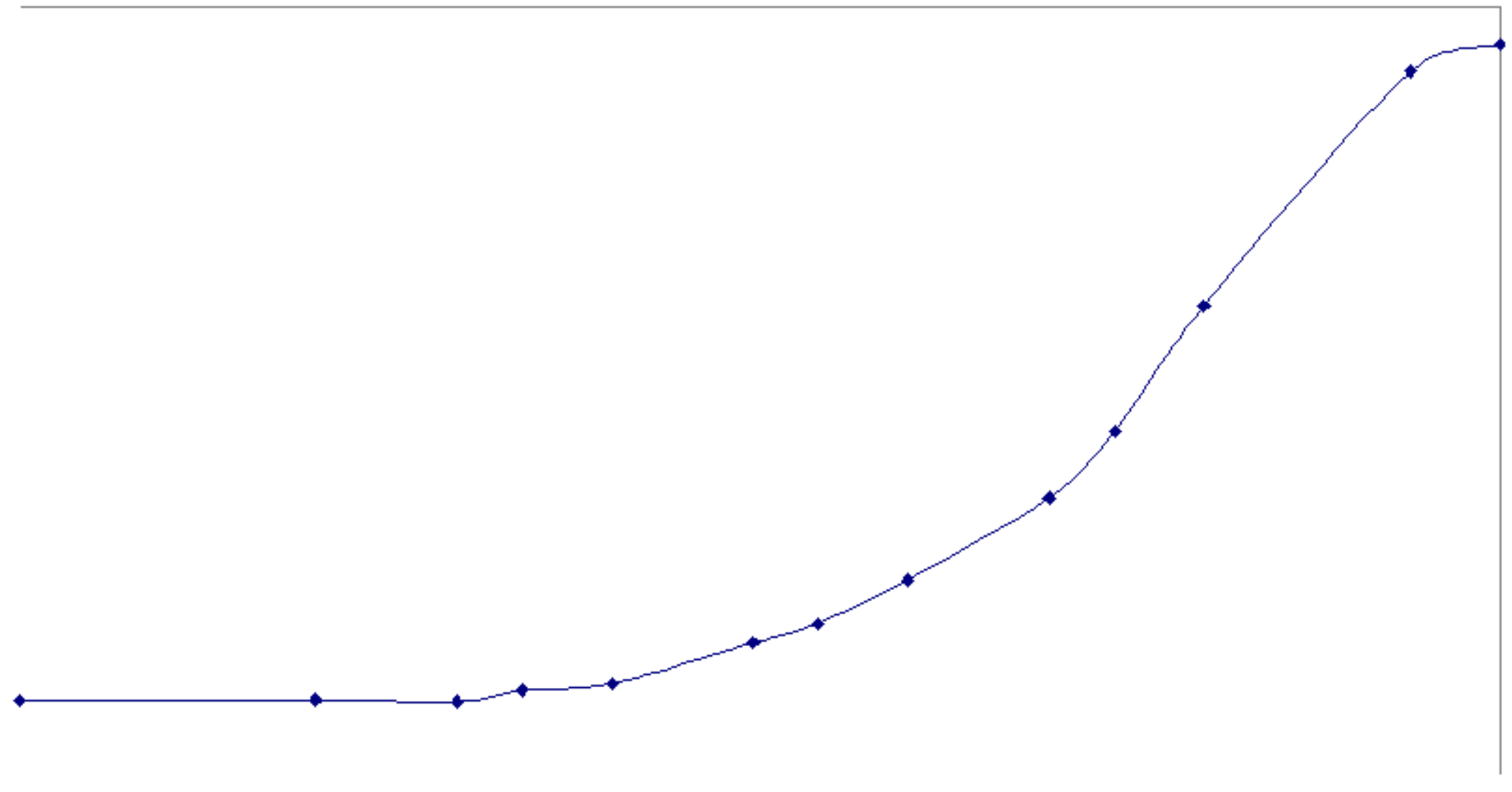


FY98 Annual Report

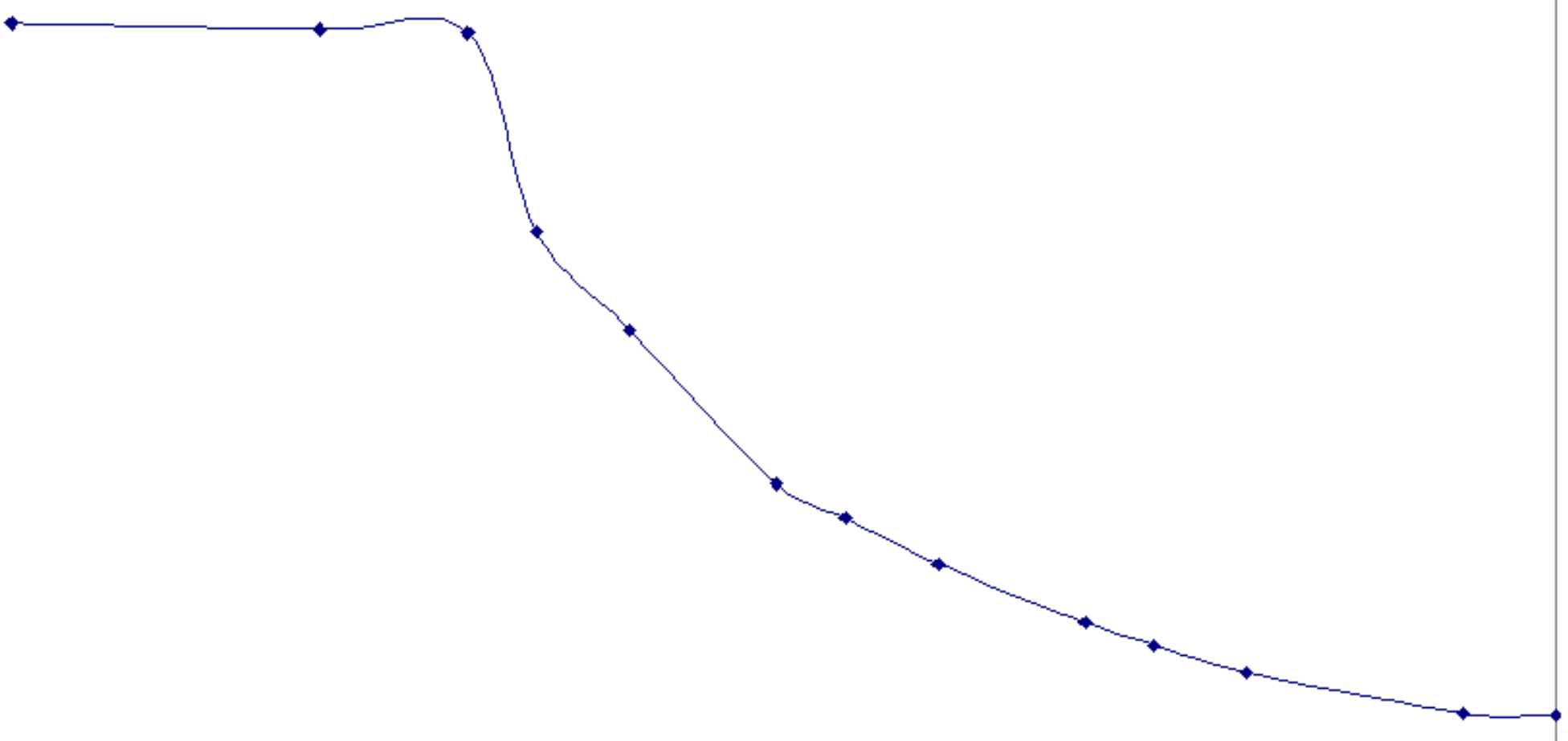

file:///C|/ManualConversion/HTML/6792benk.htm (113 of 250)4/9/2004 4:05:24 AM 

FY98 Annual Report

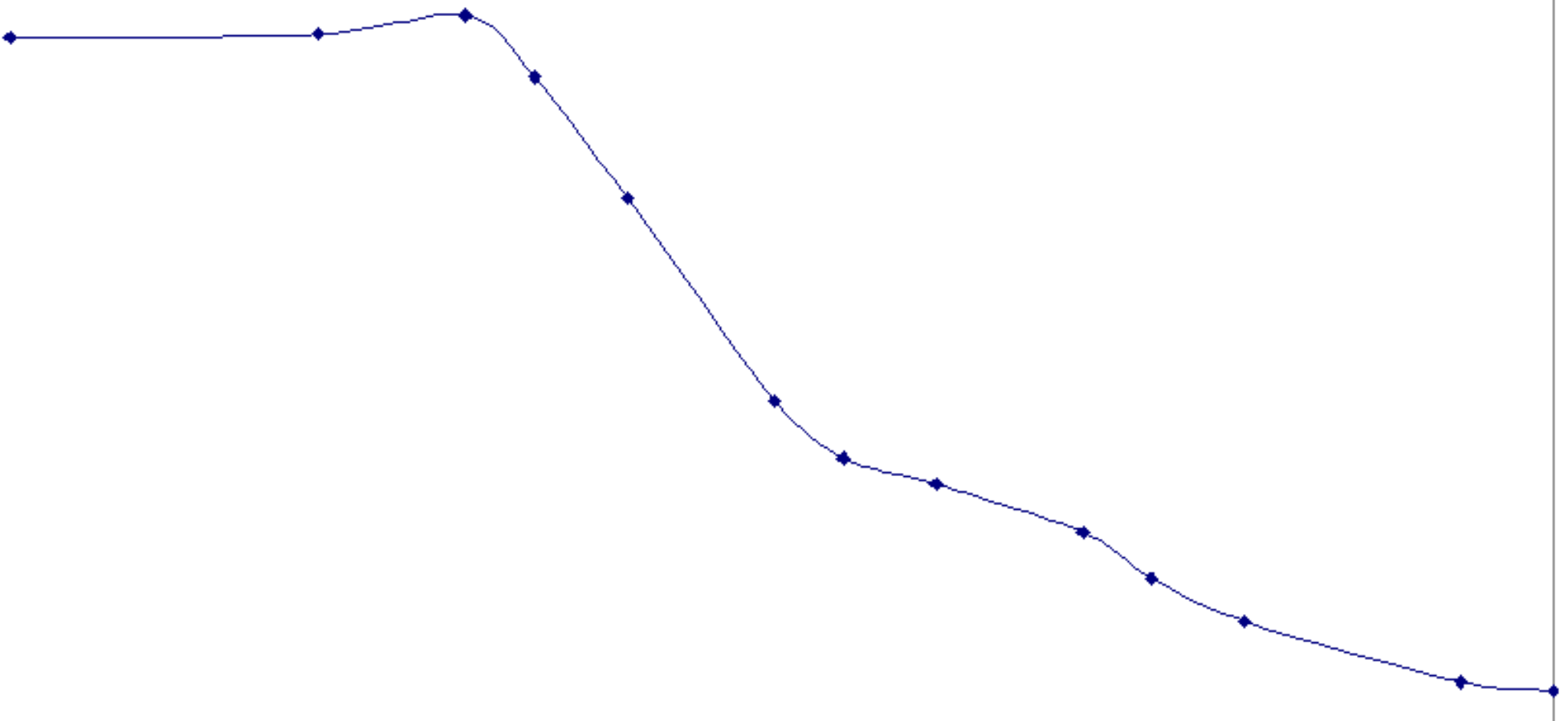

file:///C|/ManualConversion/HTML/6792benk.htm (115 of 250)4/9/2004 4:05:24 AM 


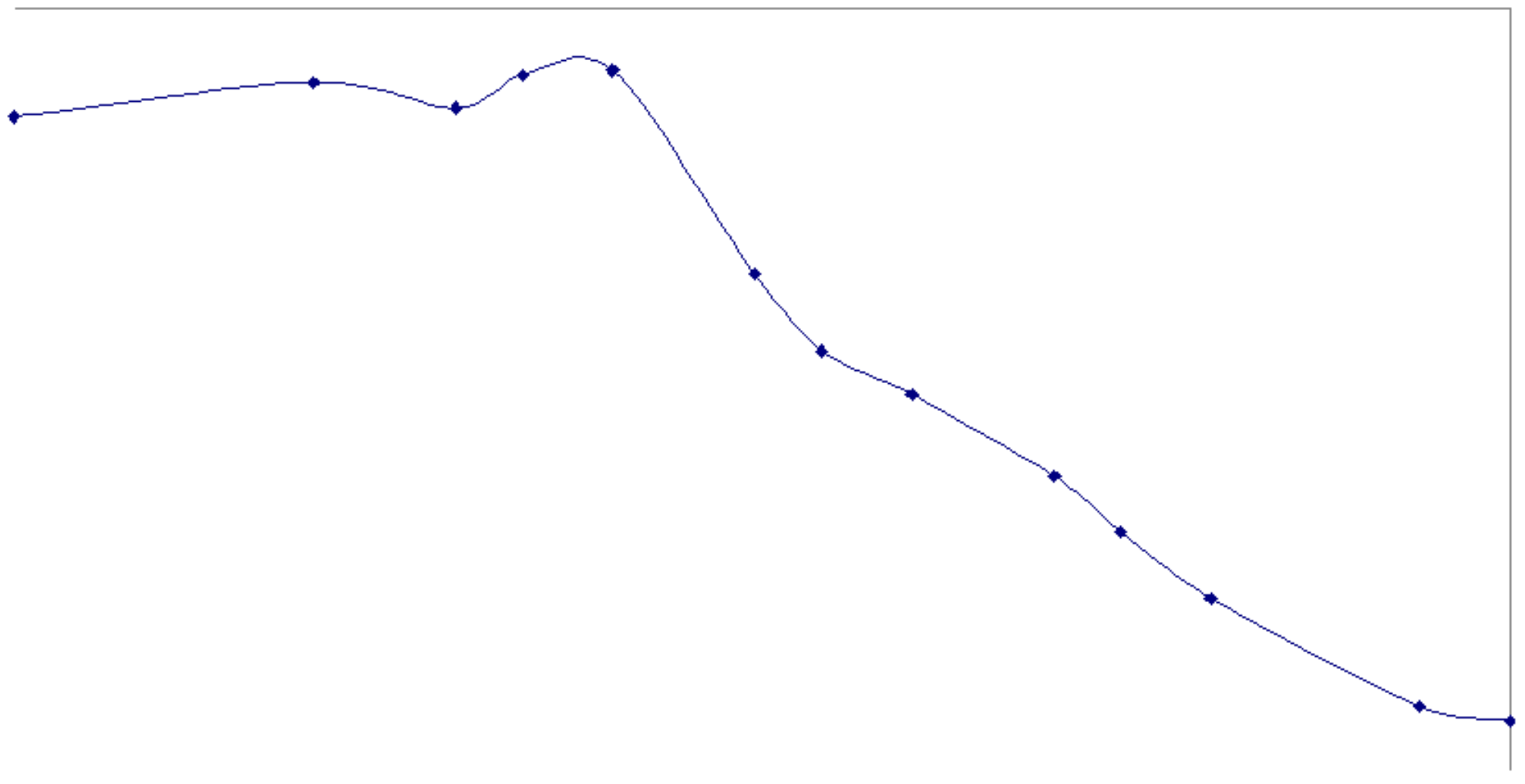




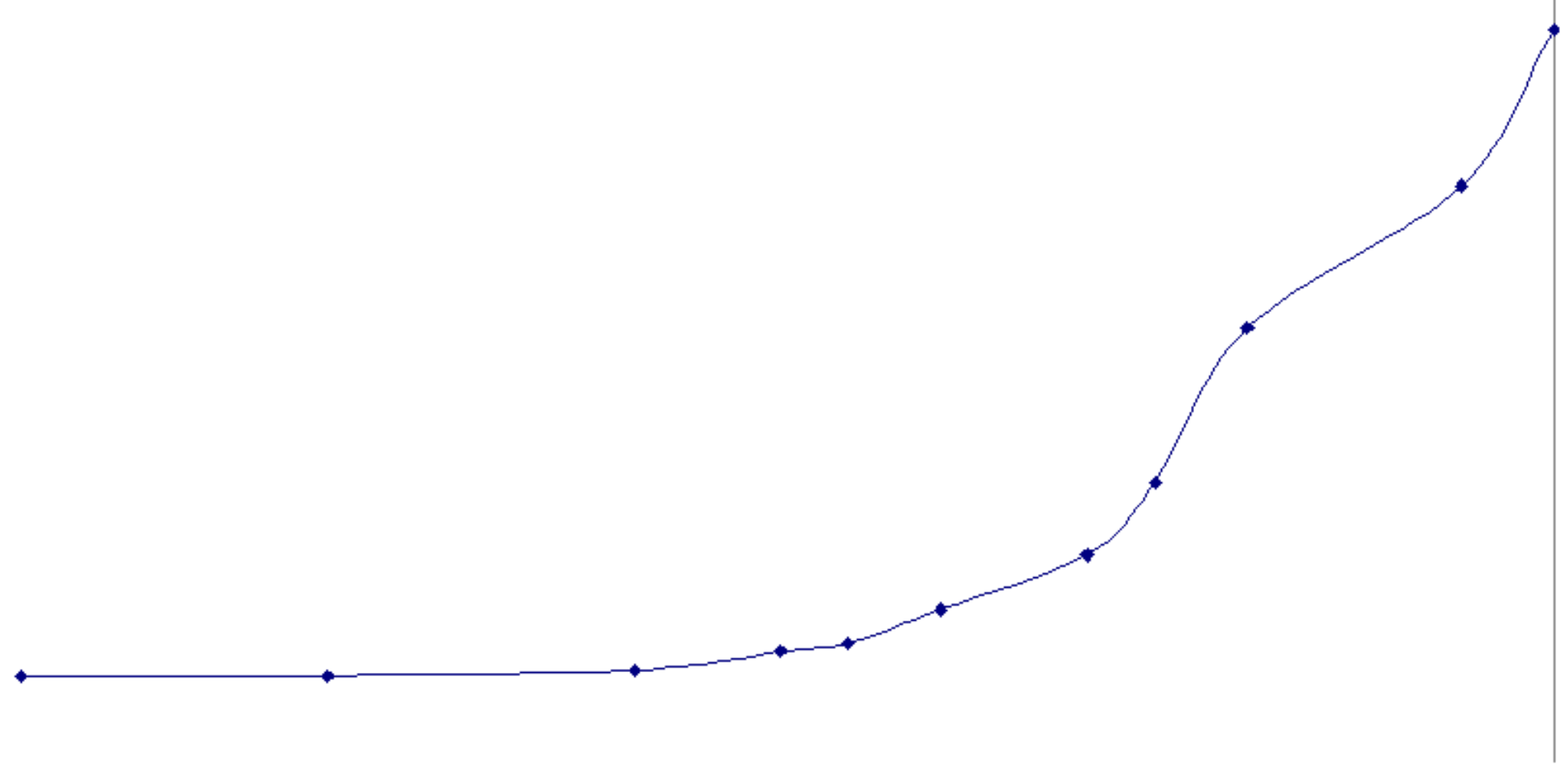




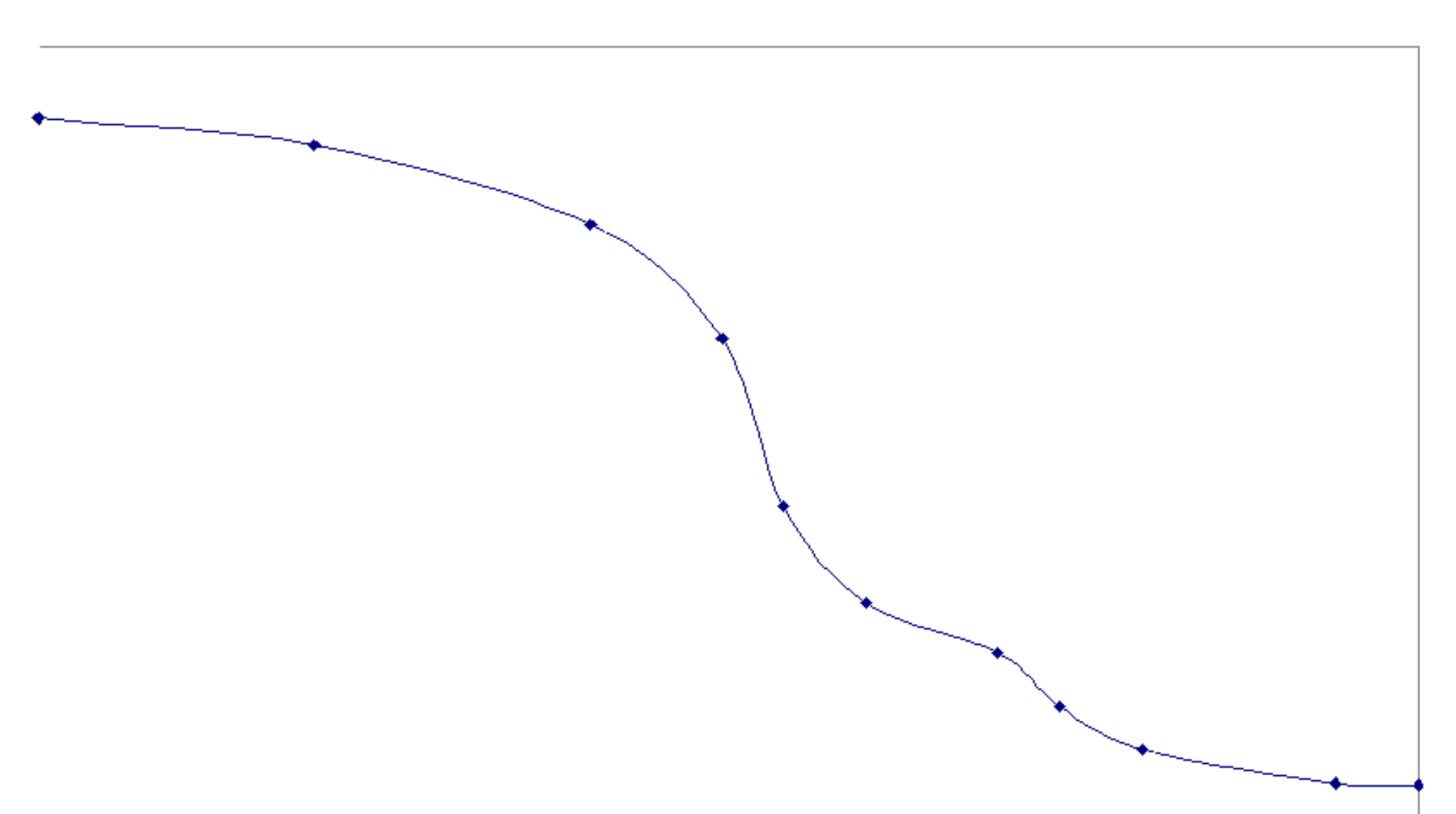



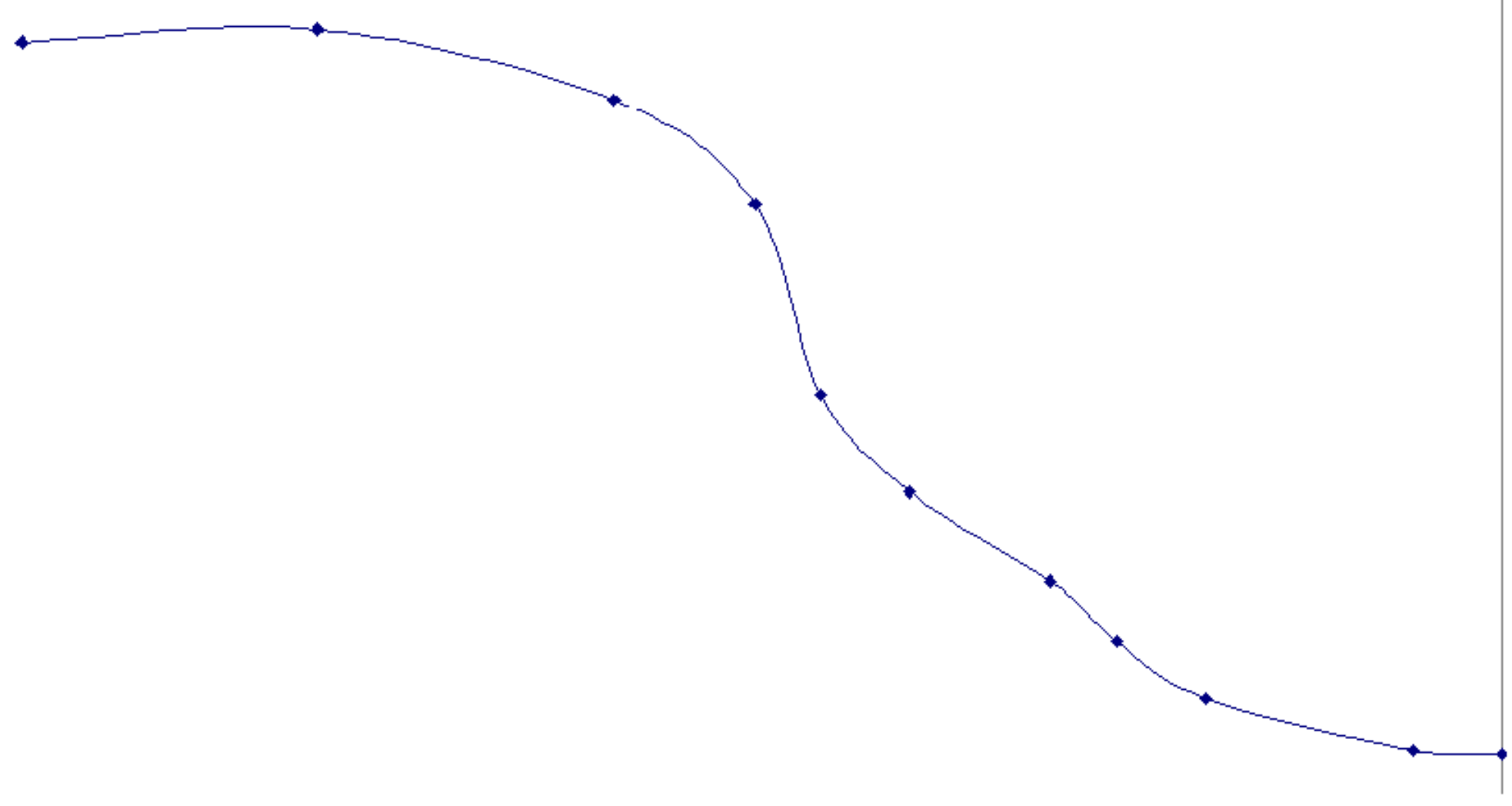


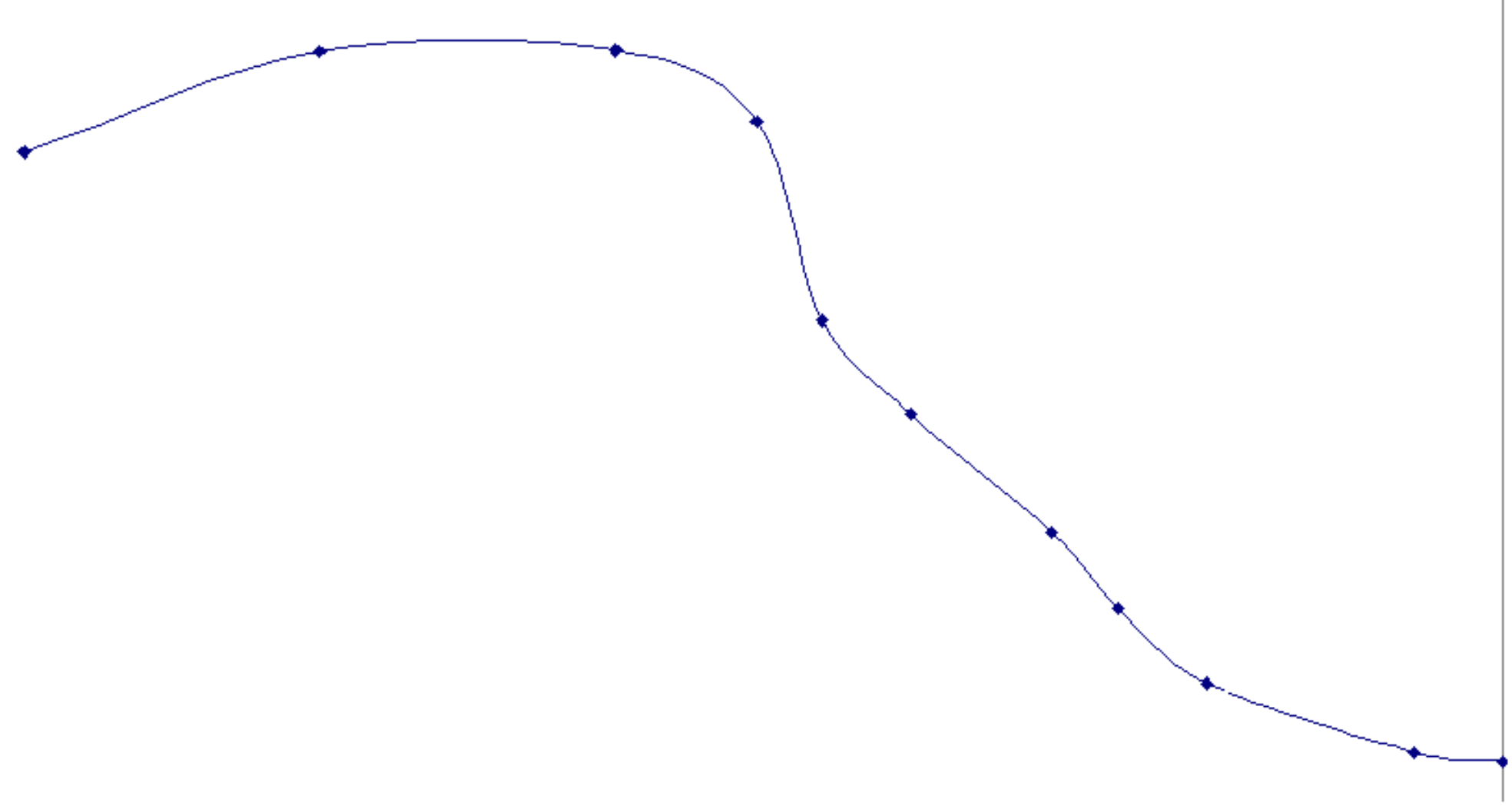




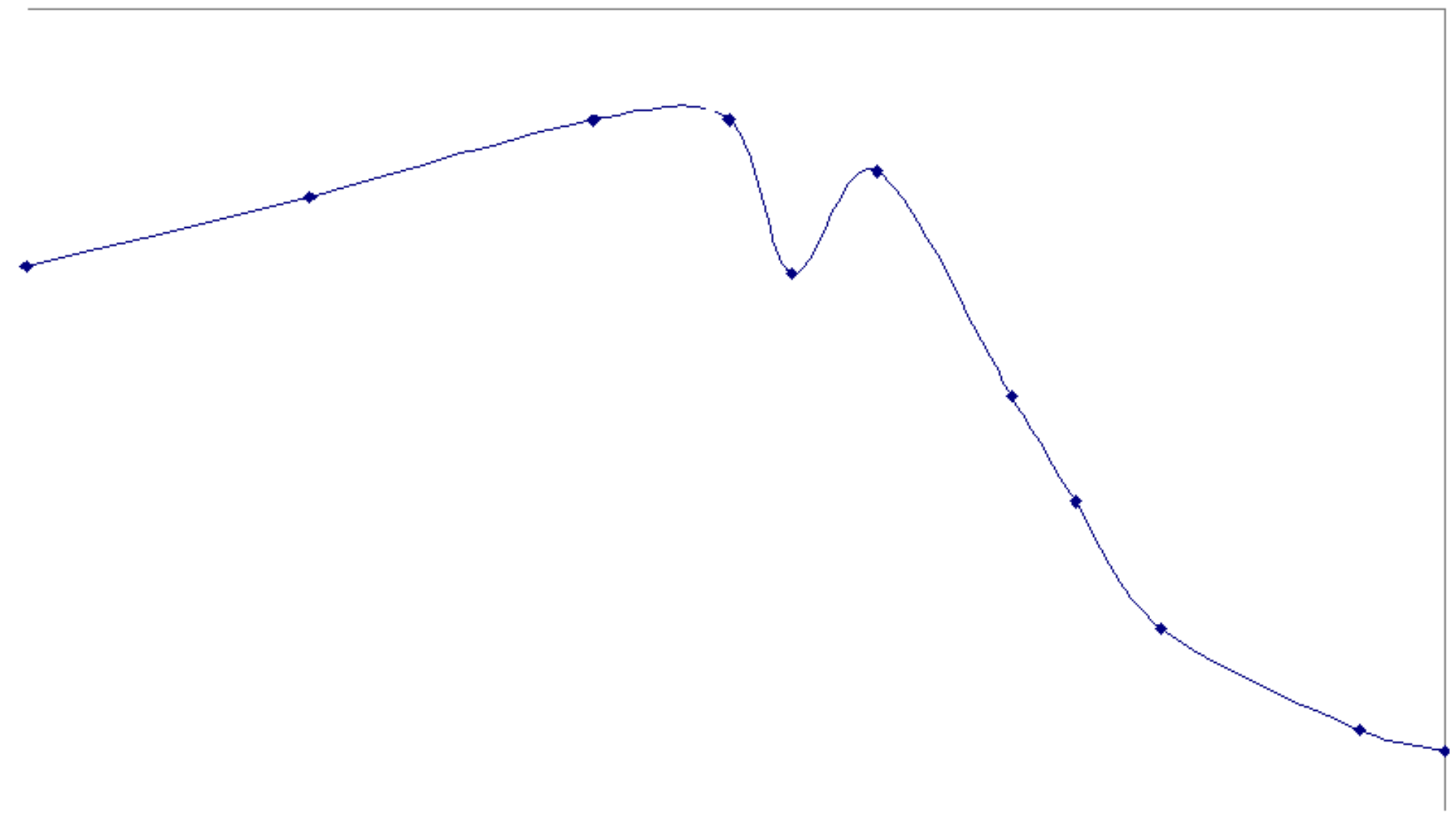




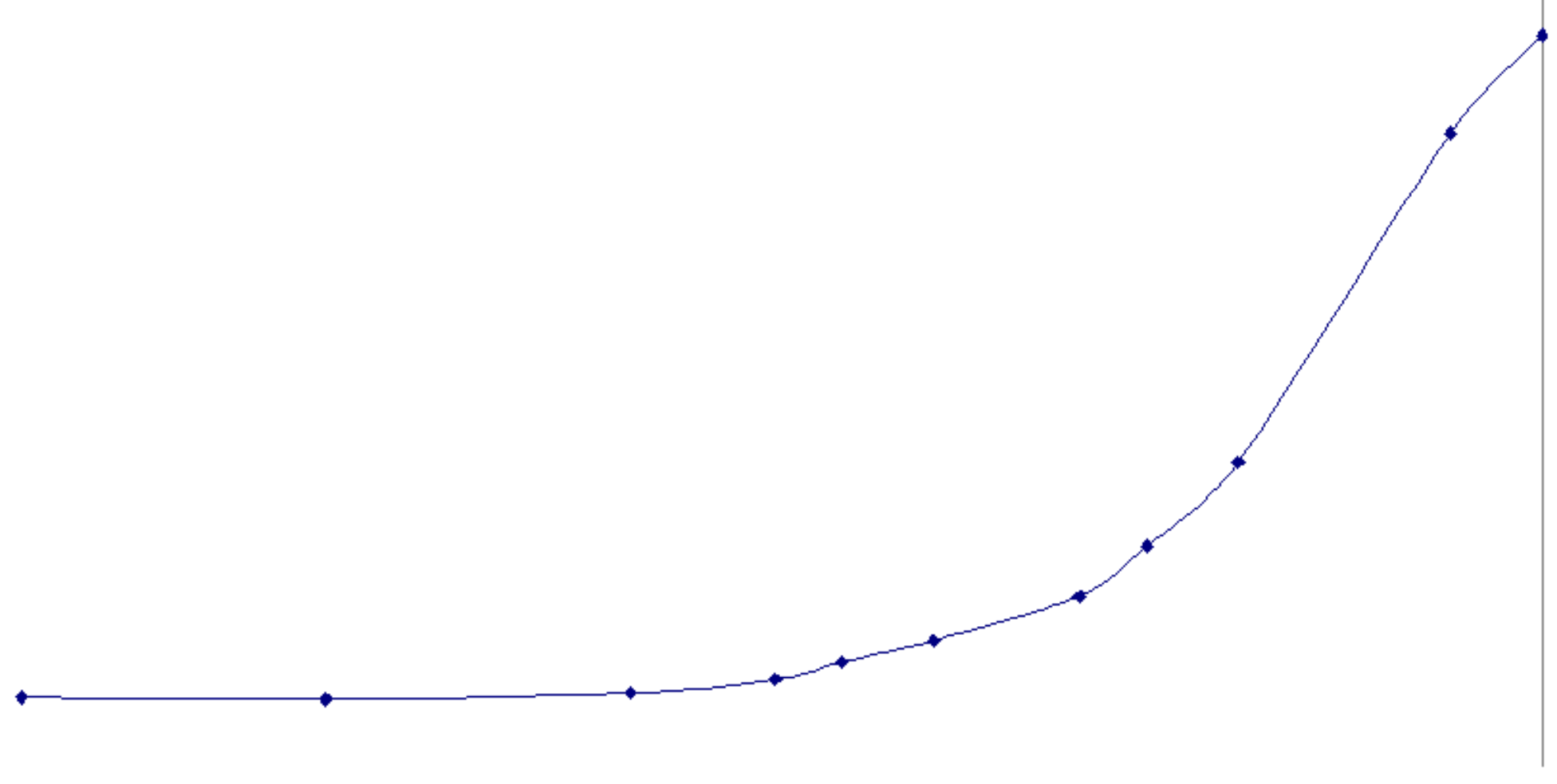




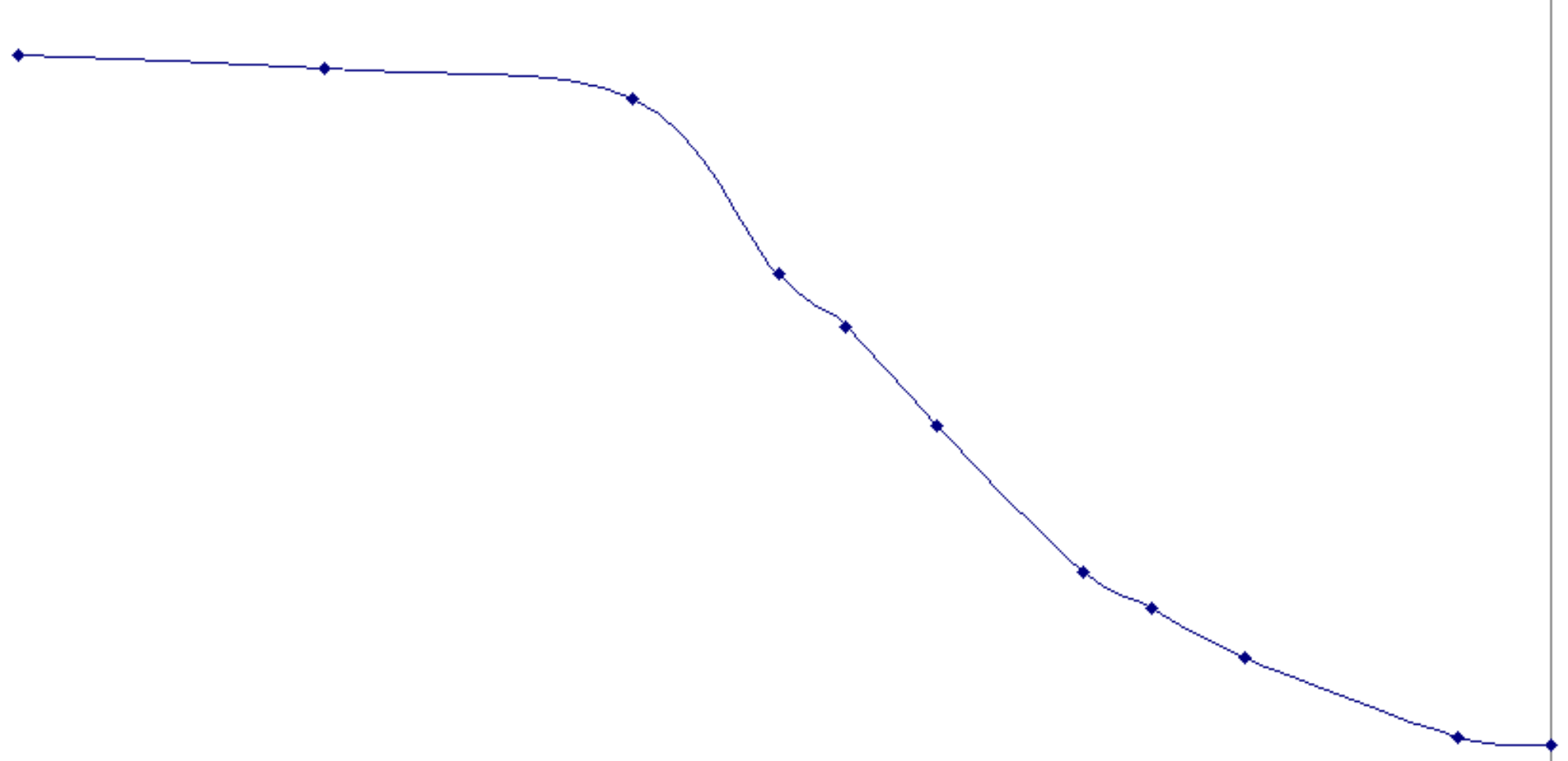




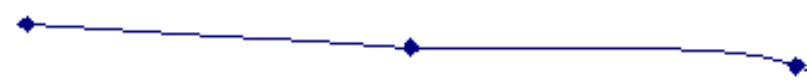

$+$

$-$

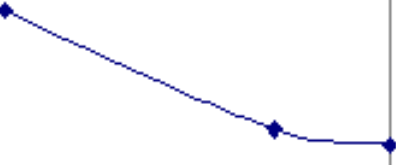




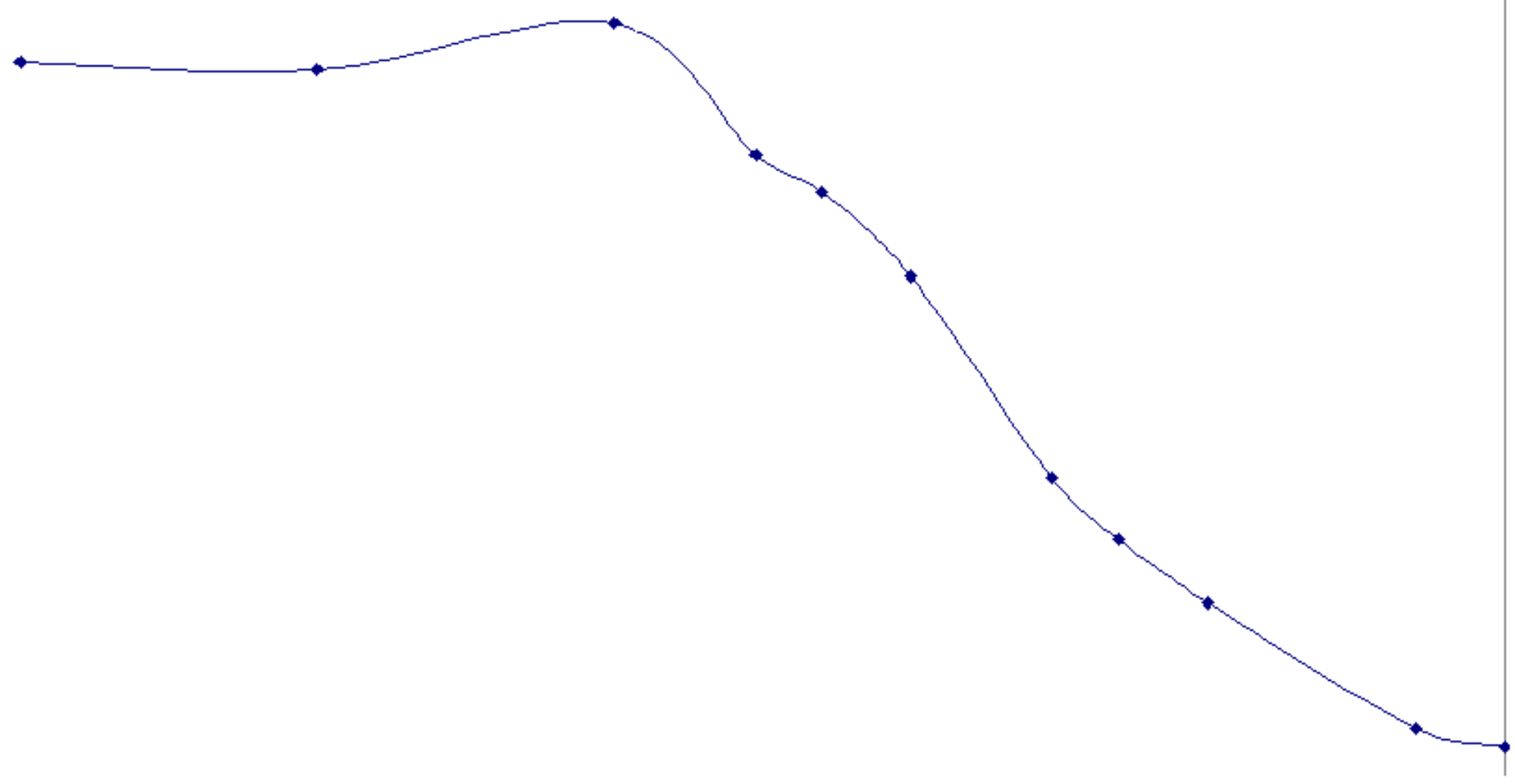




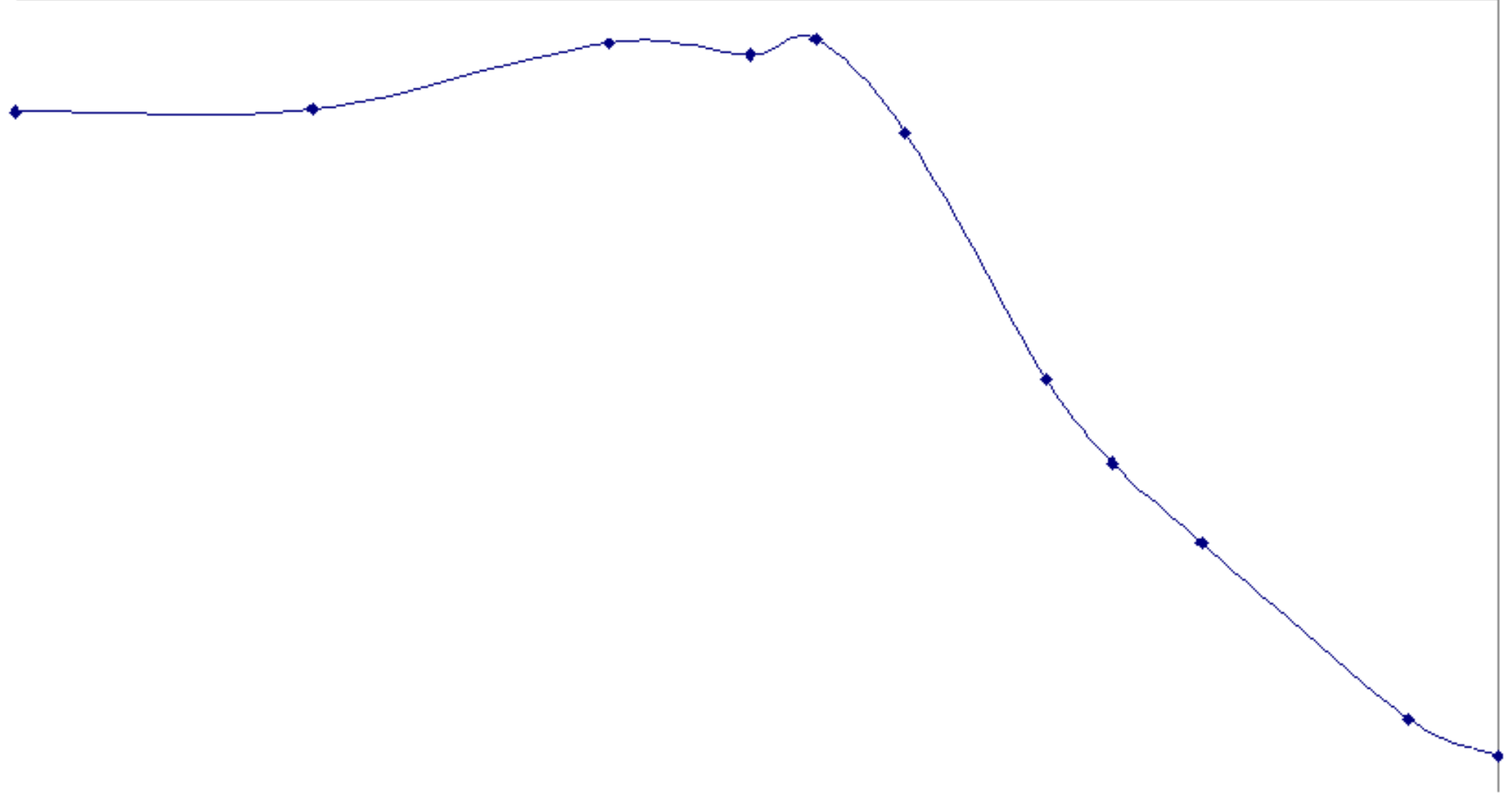




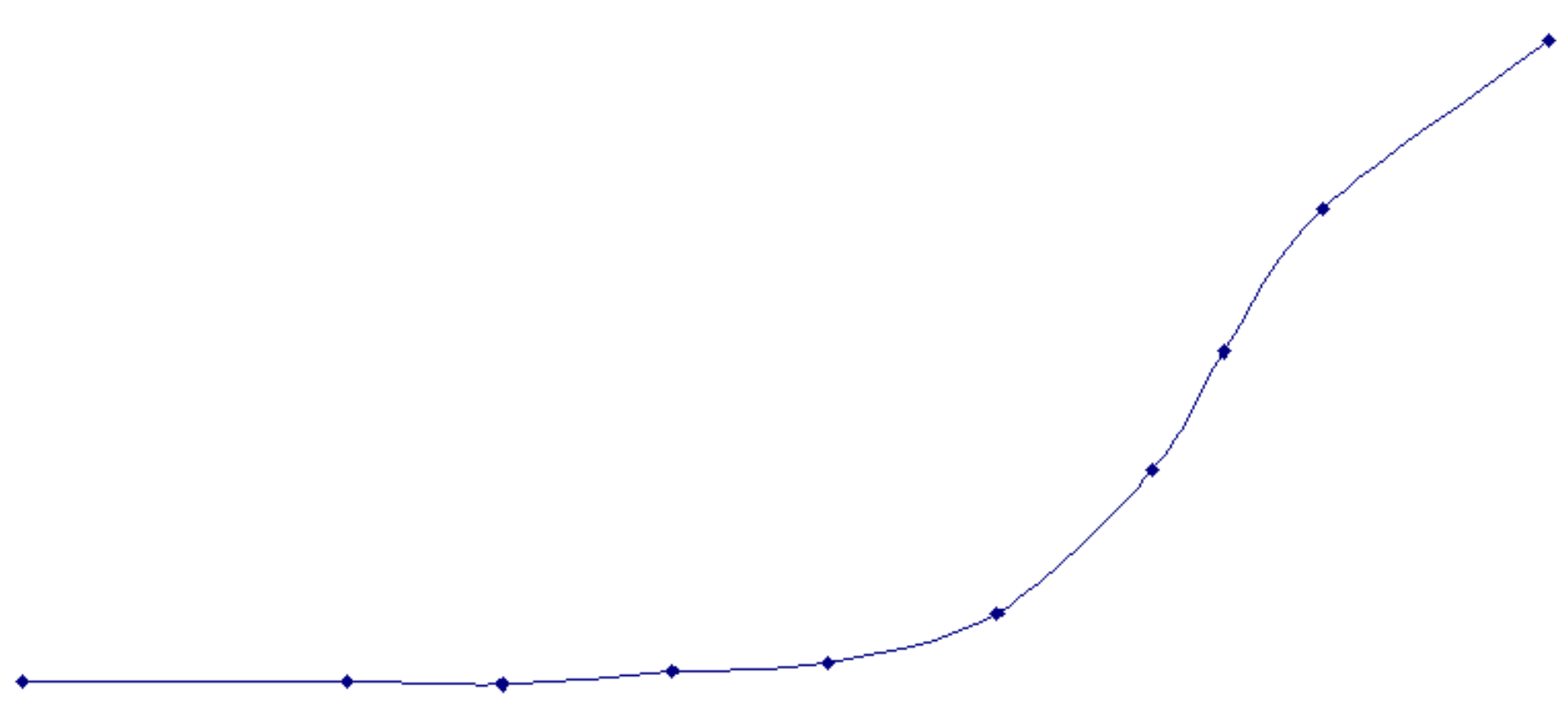




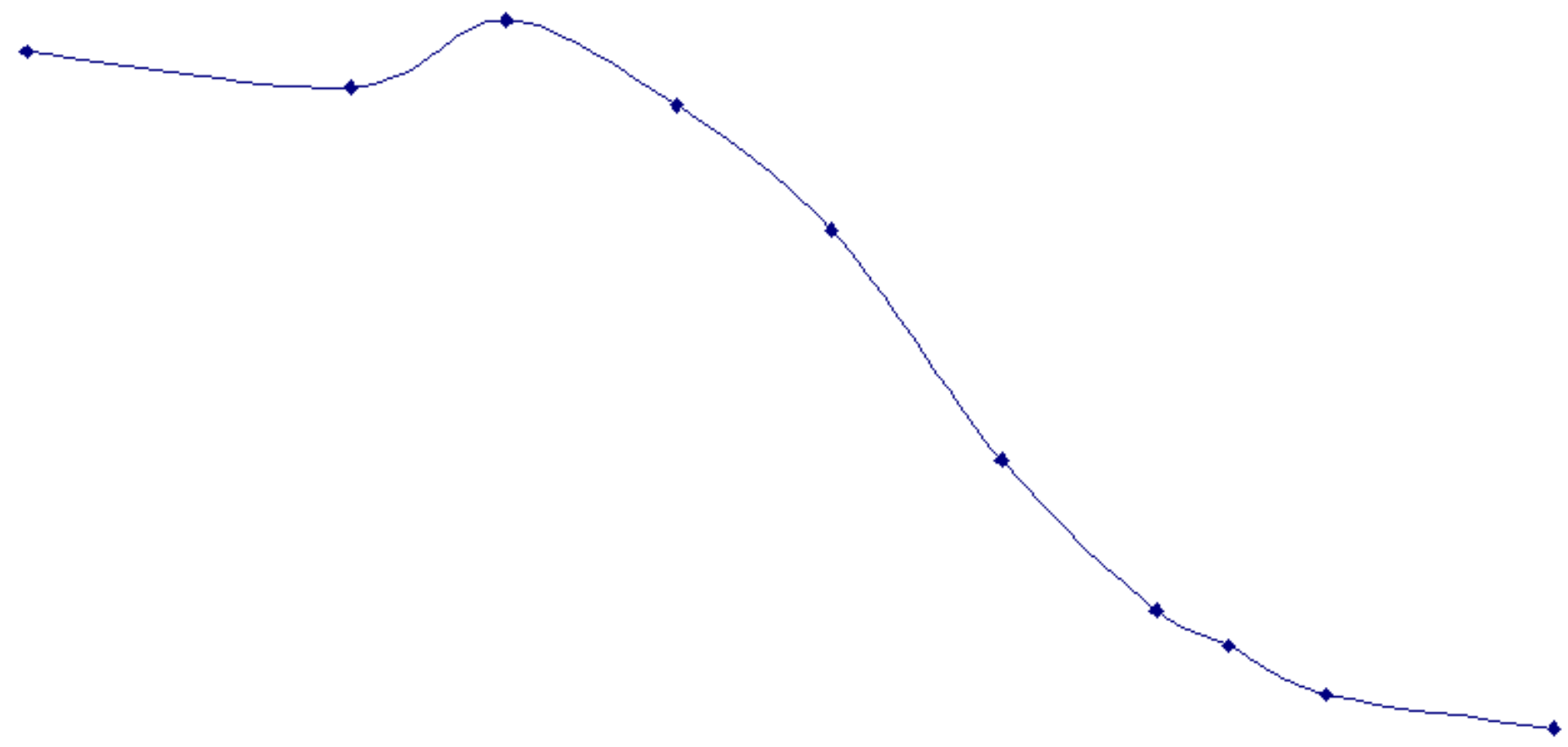




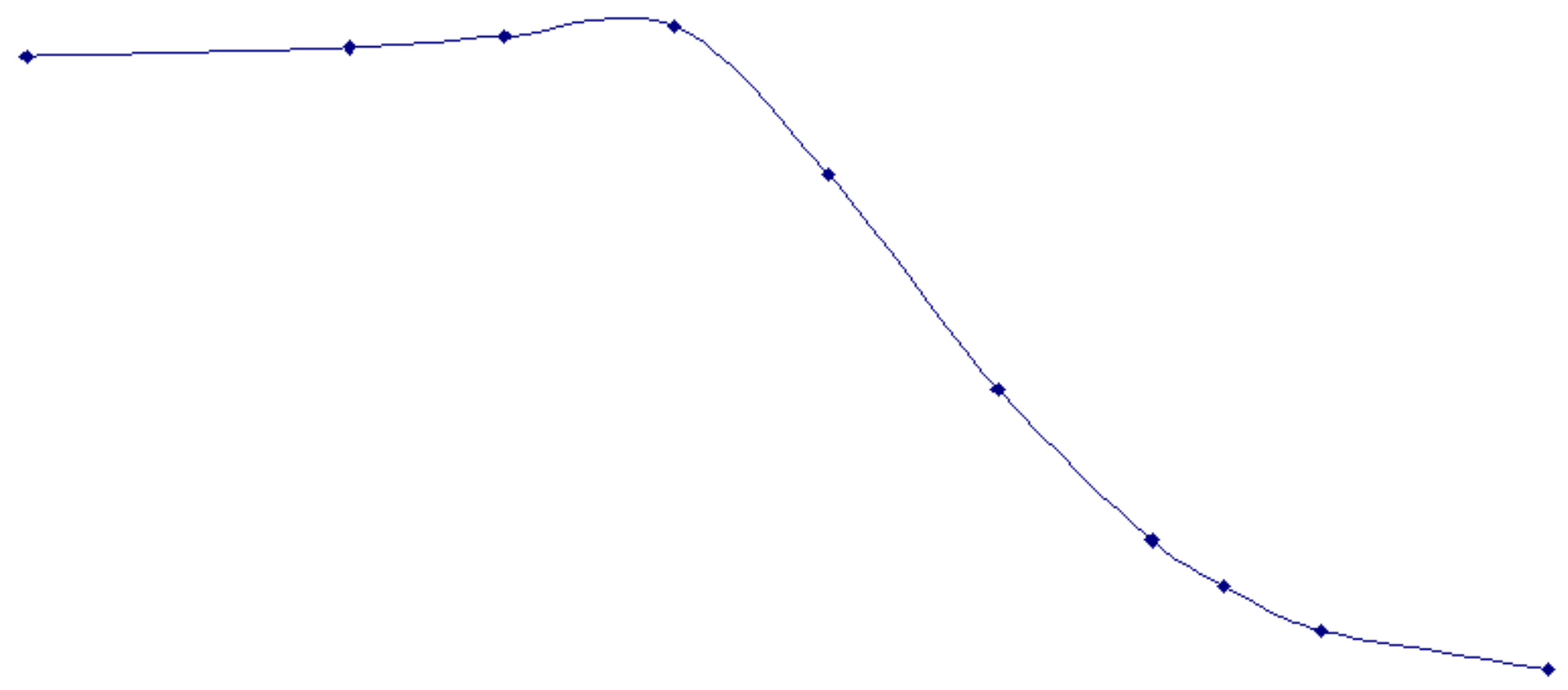




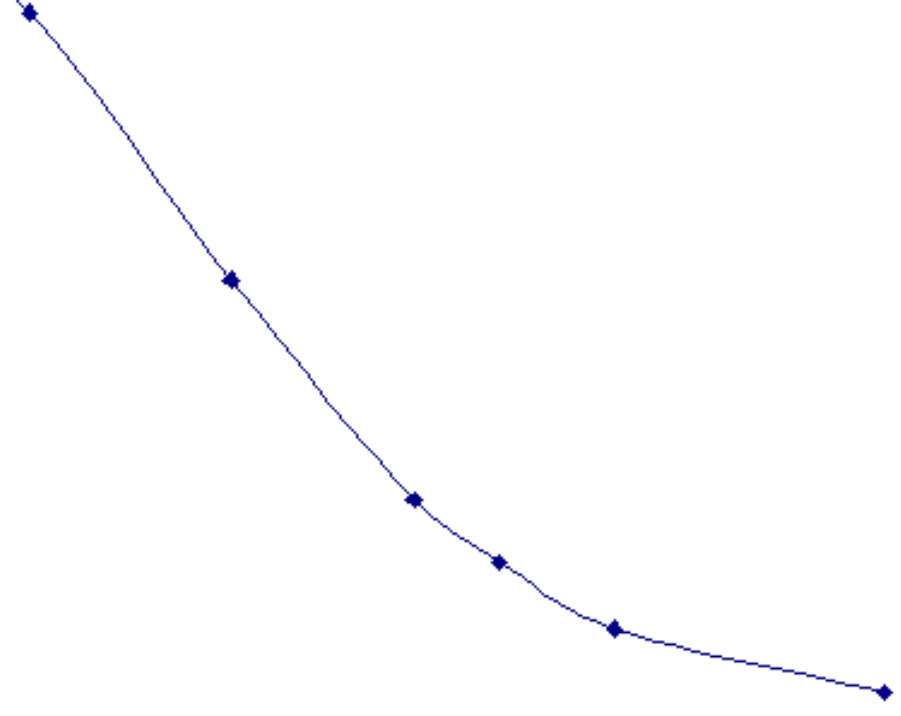




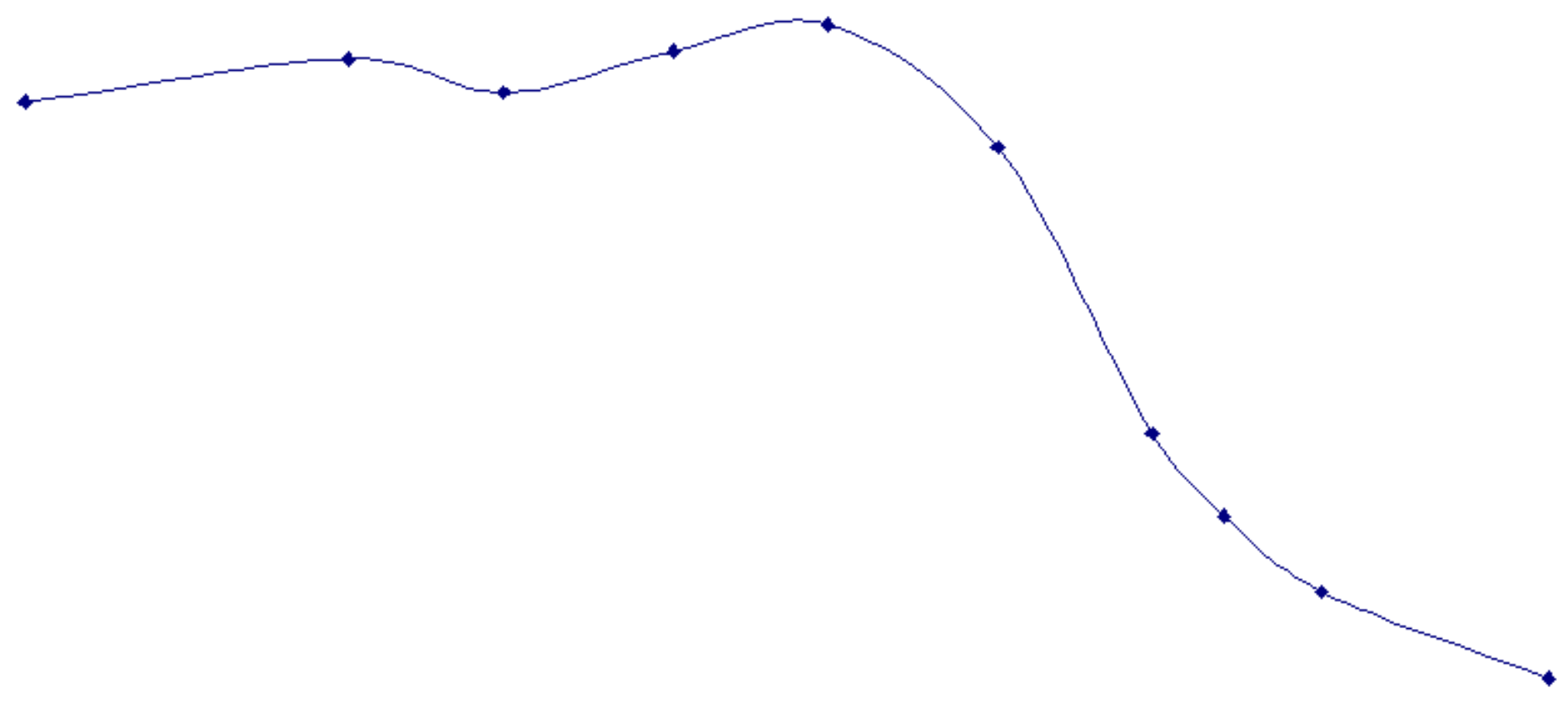


<smiles>CCCCCC</smiles> 
FY98 Annual Report

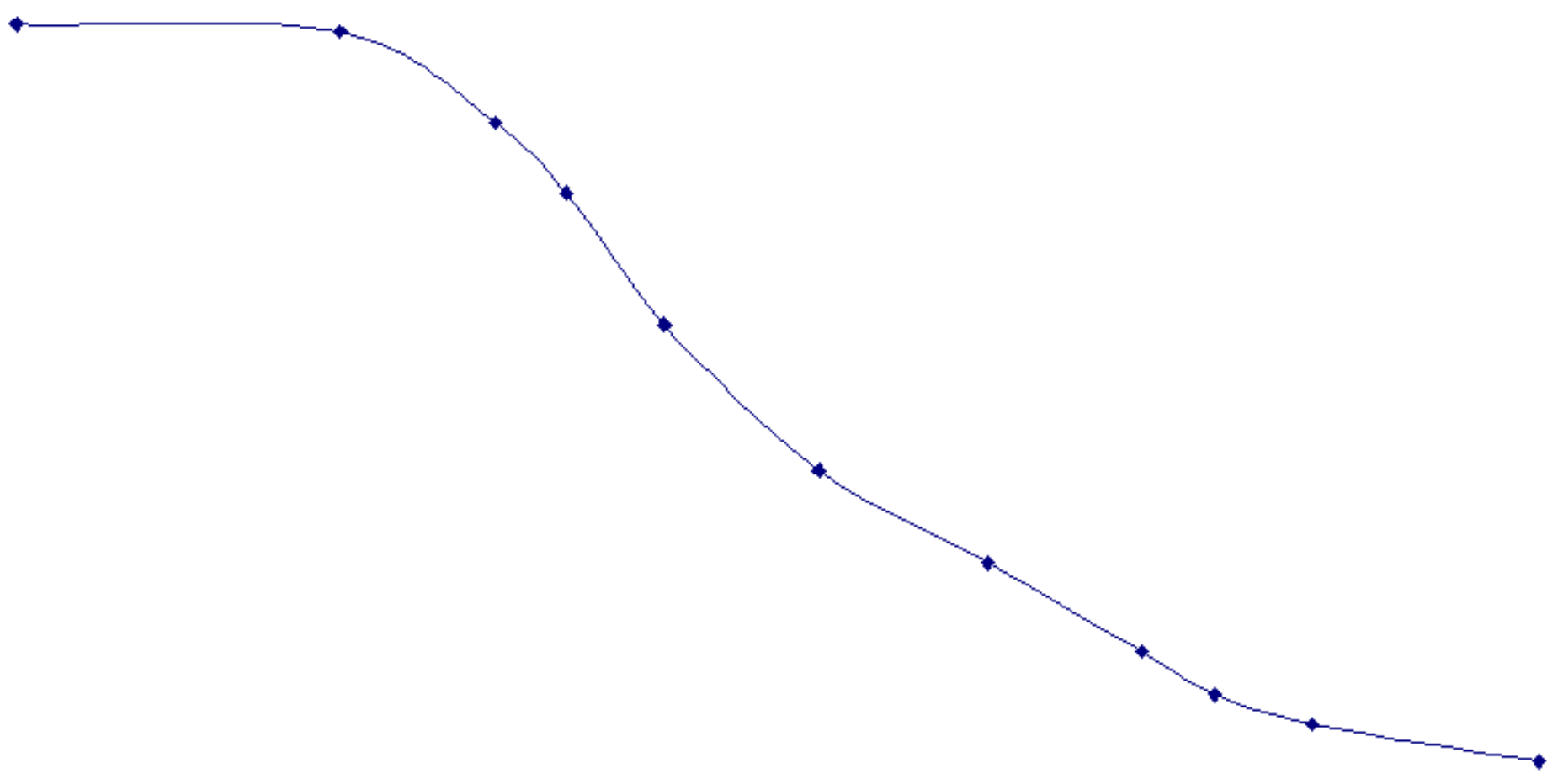

file://C//ManualConversion/HTML/6792benk.htm (133 of 250)4/9/2004 4:05:24 AM 
<smiles>C=CCC1CCC1CC</smiles> 

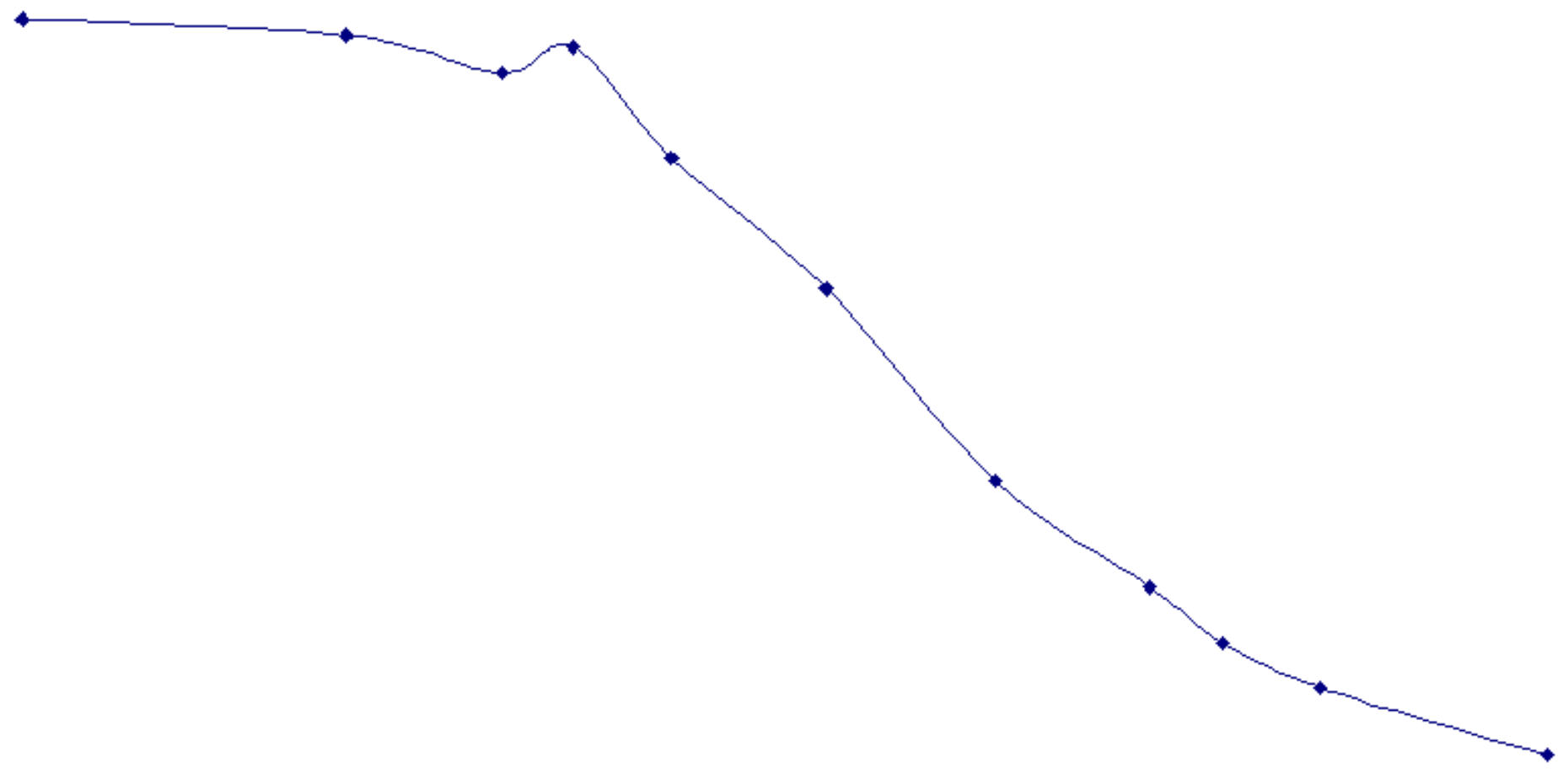


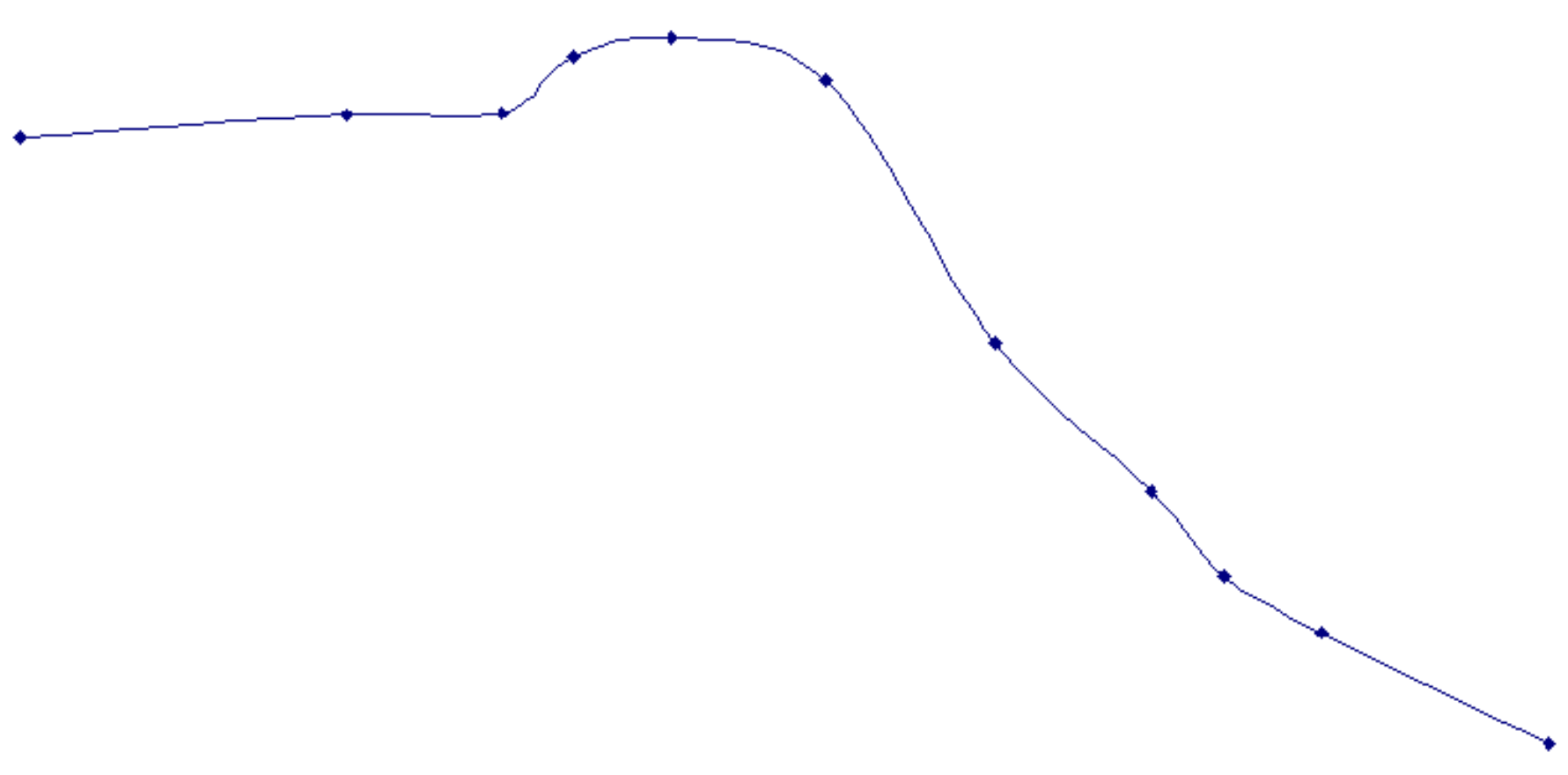


Appendix I

Calibration Curves for Dioctyl Phthalate (DOP) on Aluminum Panels and Stainless Steel Disks 
FY98 Annual Report

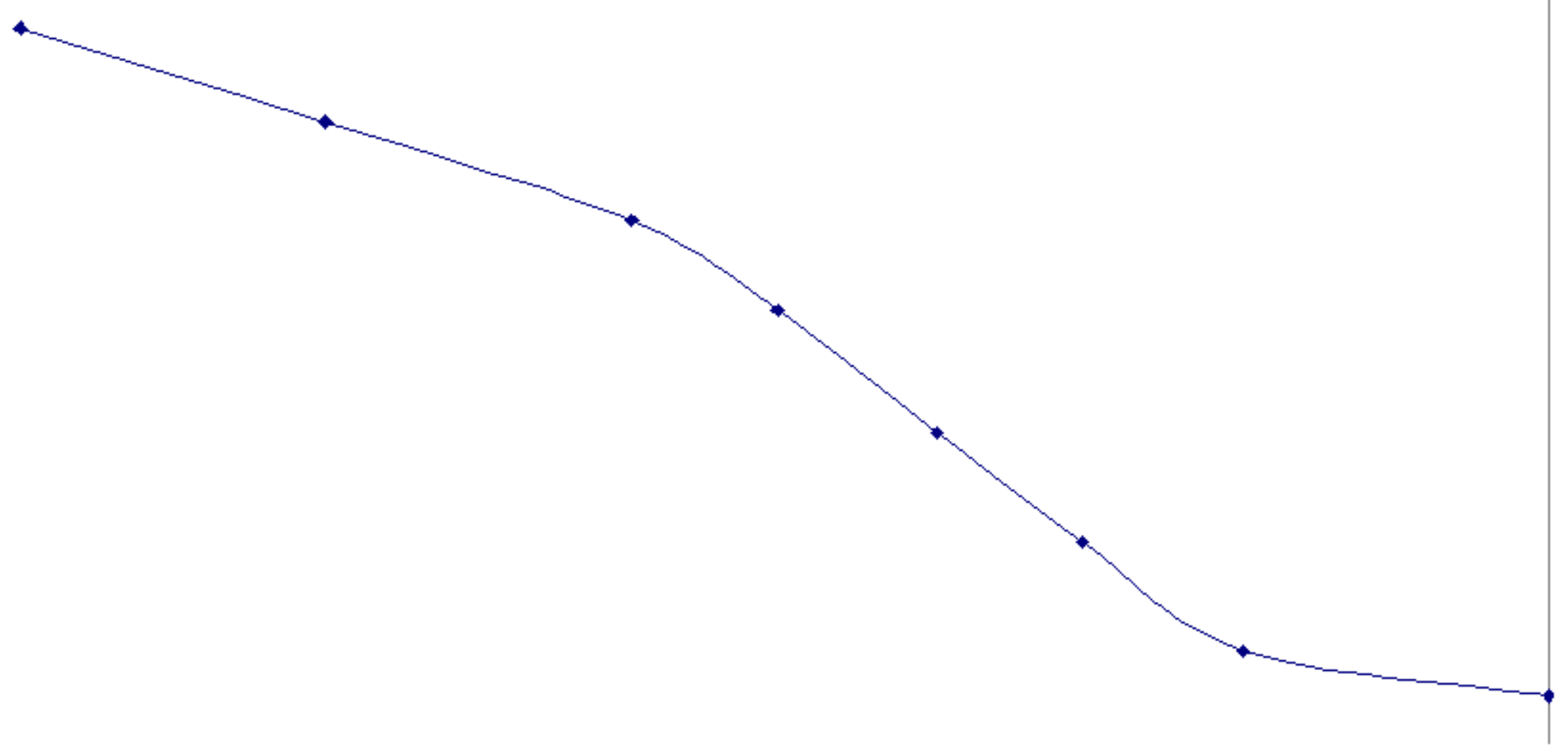

file://C//ManualConversion/HTML/6792benk.htm (138 of 250)4/9/2004 4:05:24 AM 
a
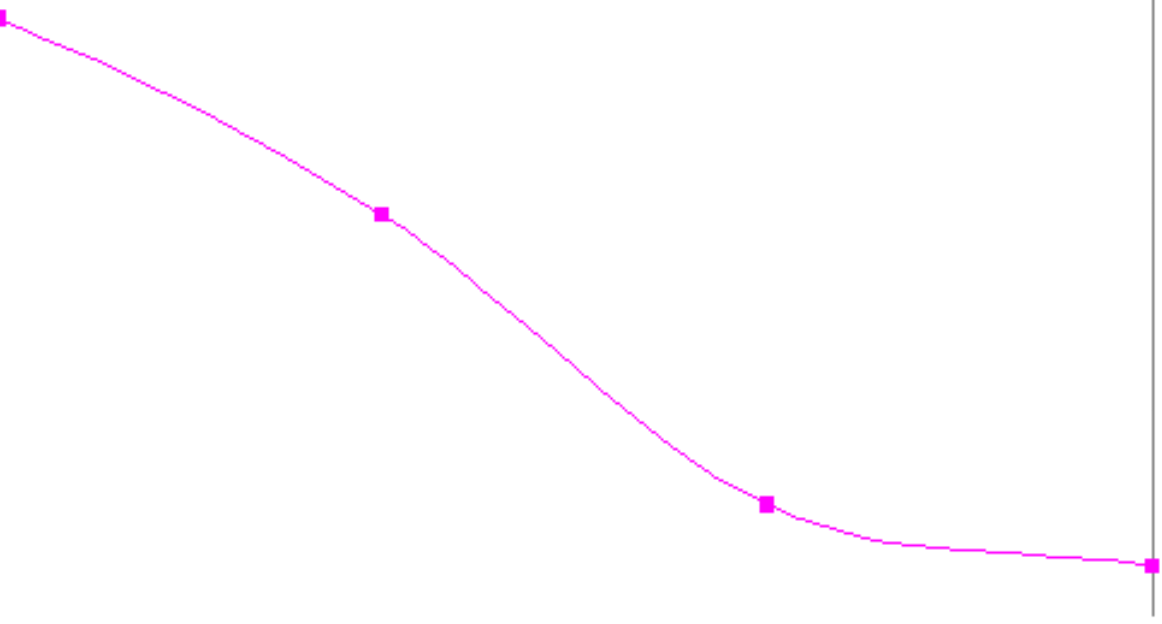


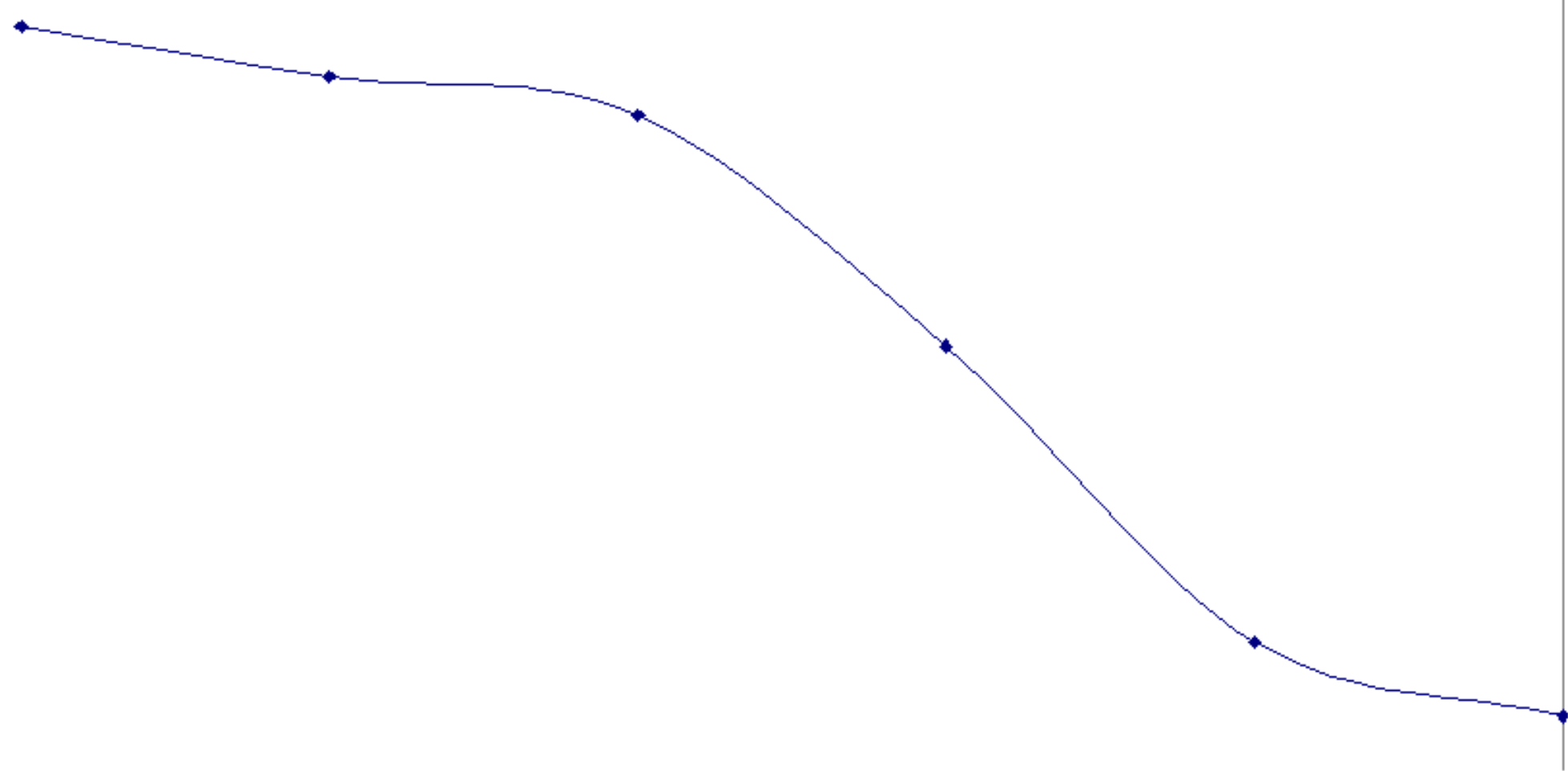




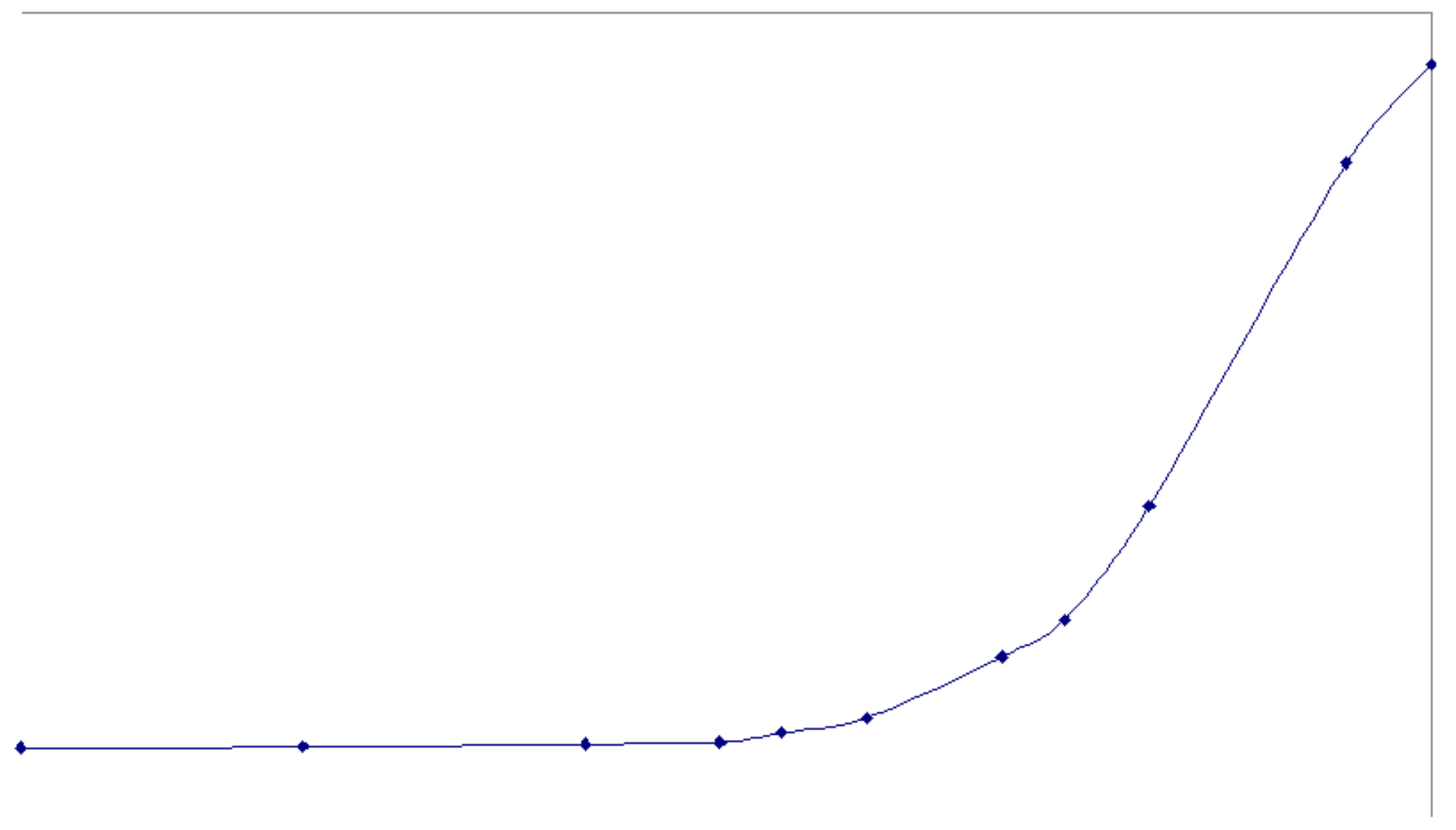




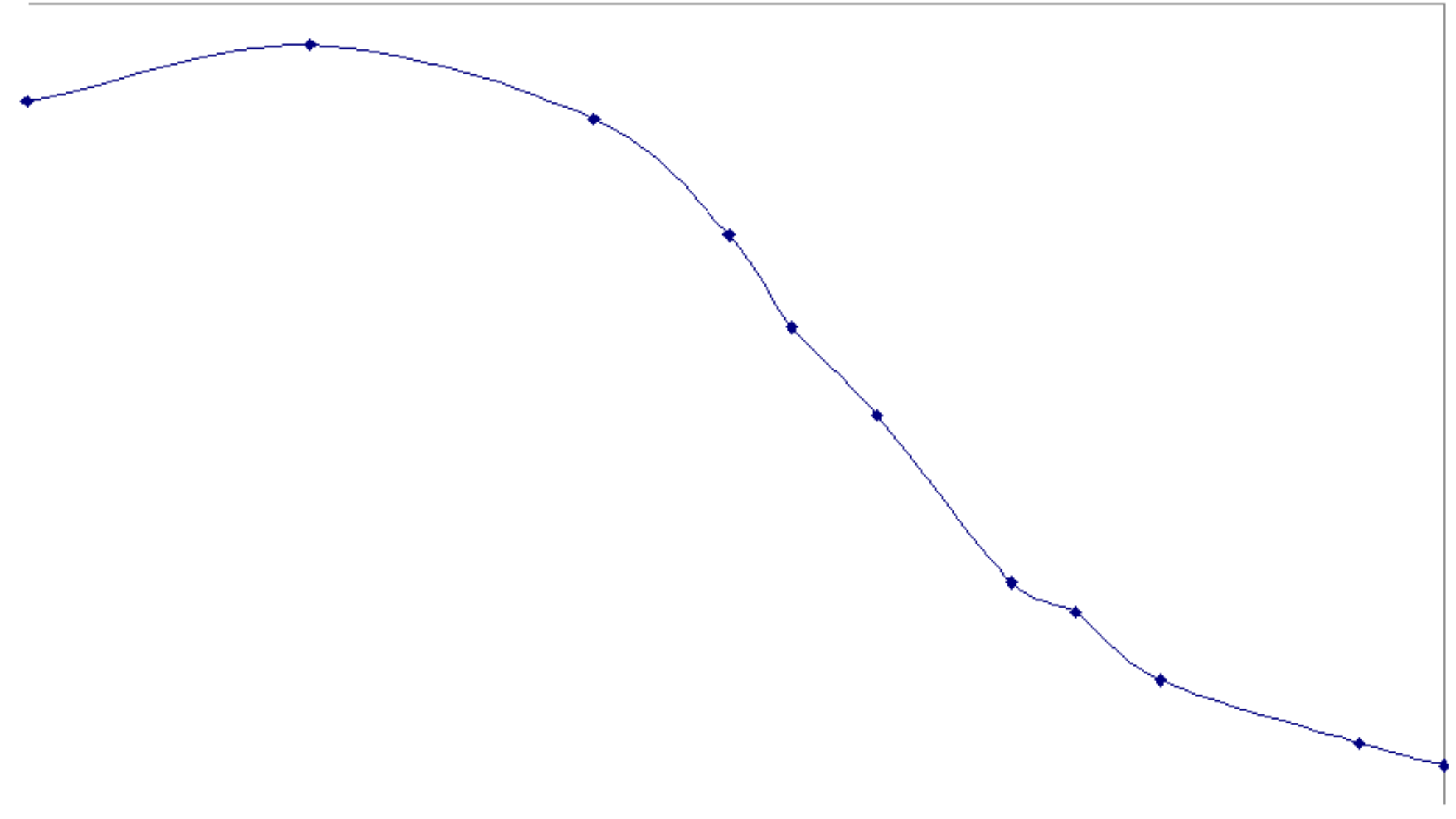




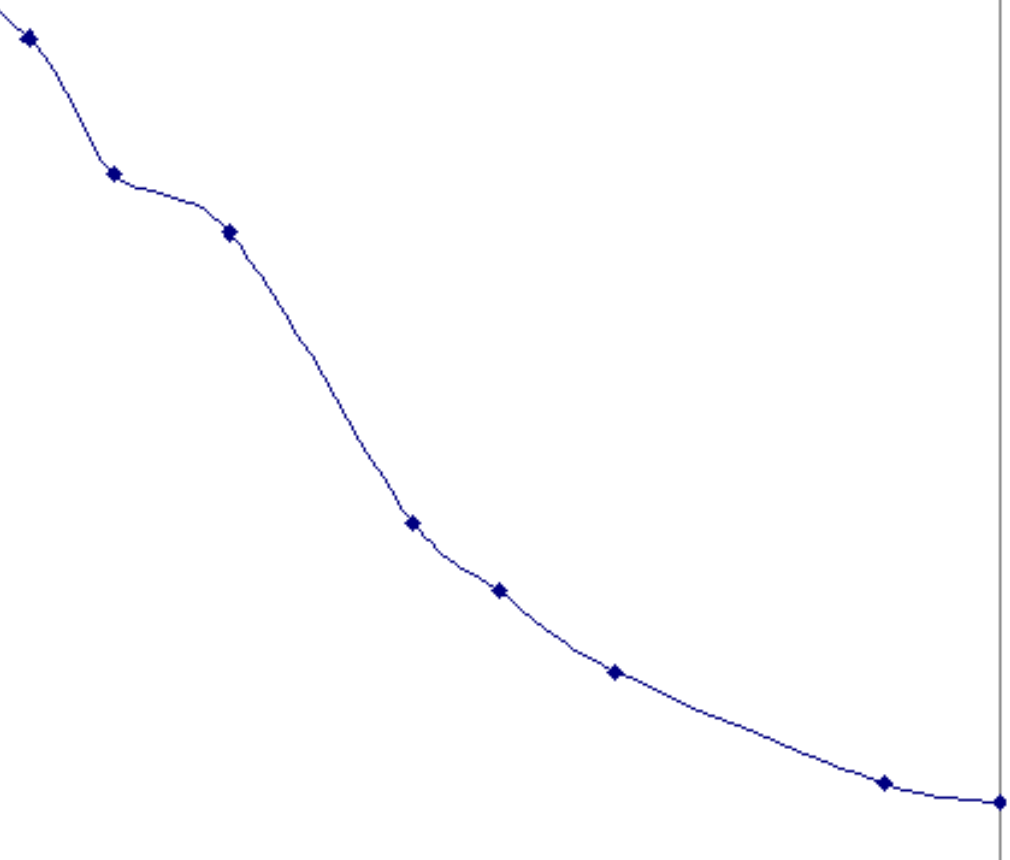




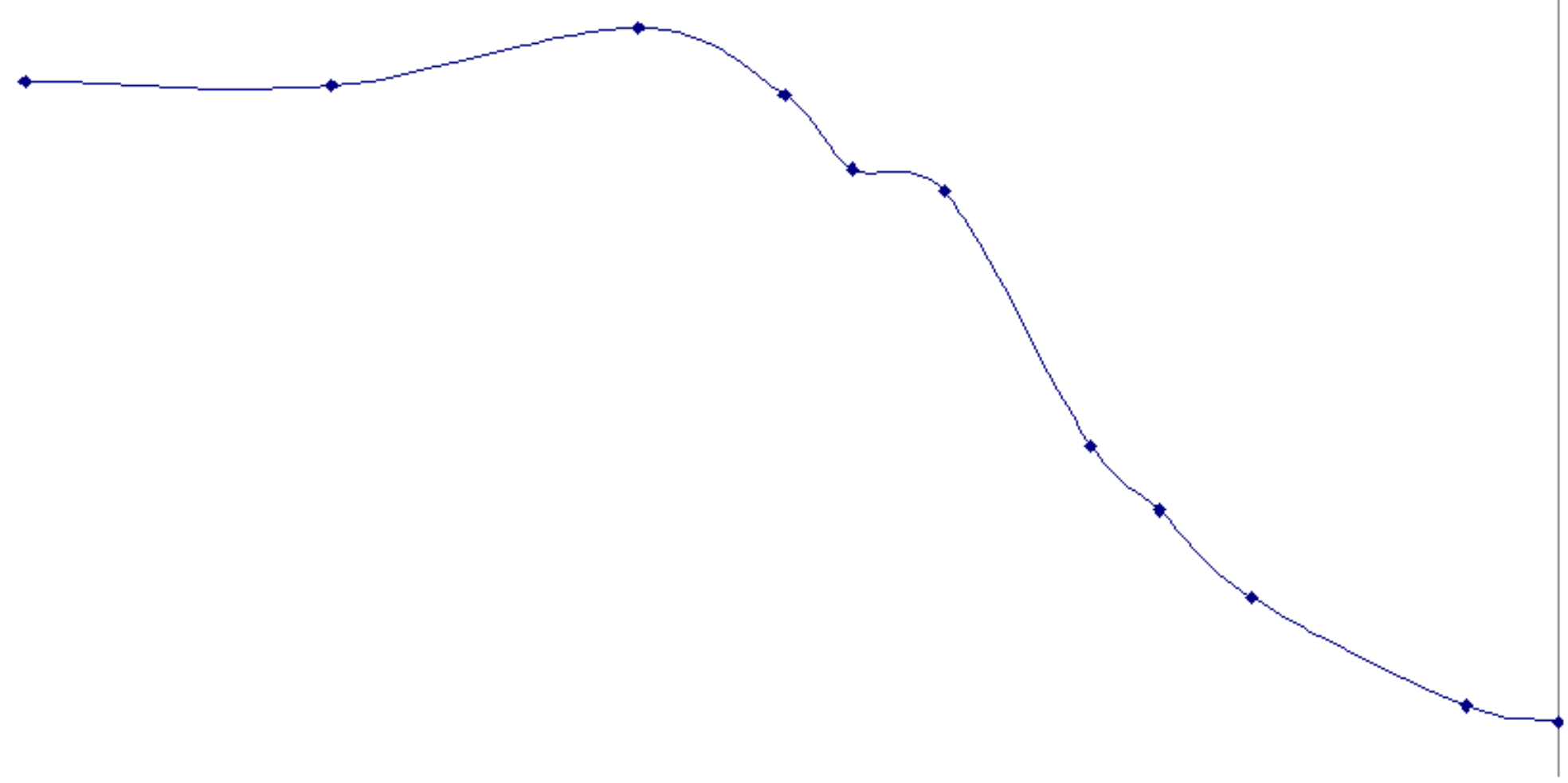




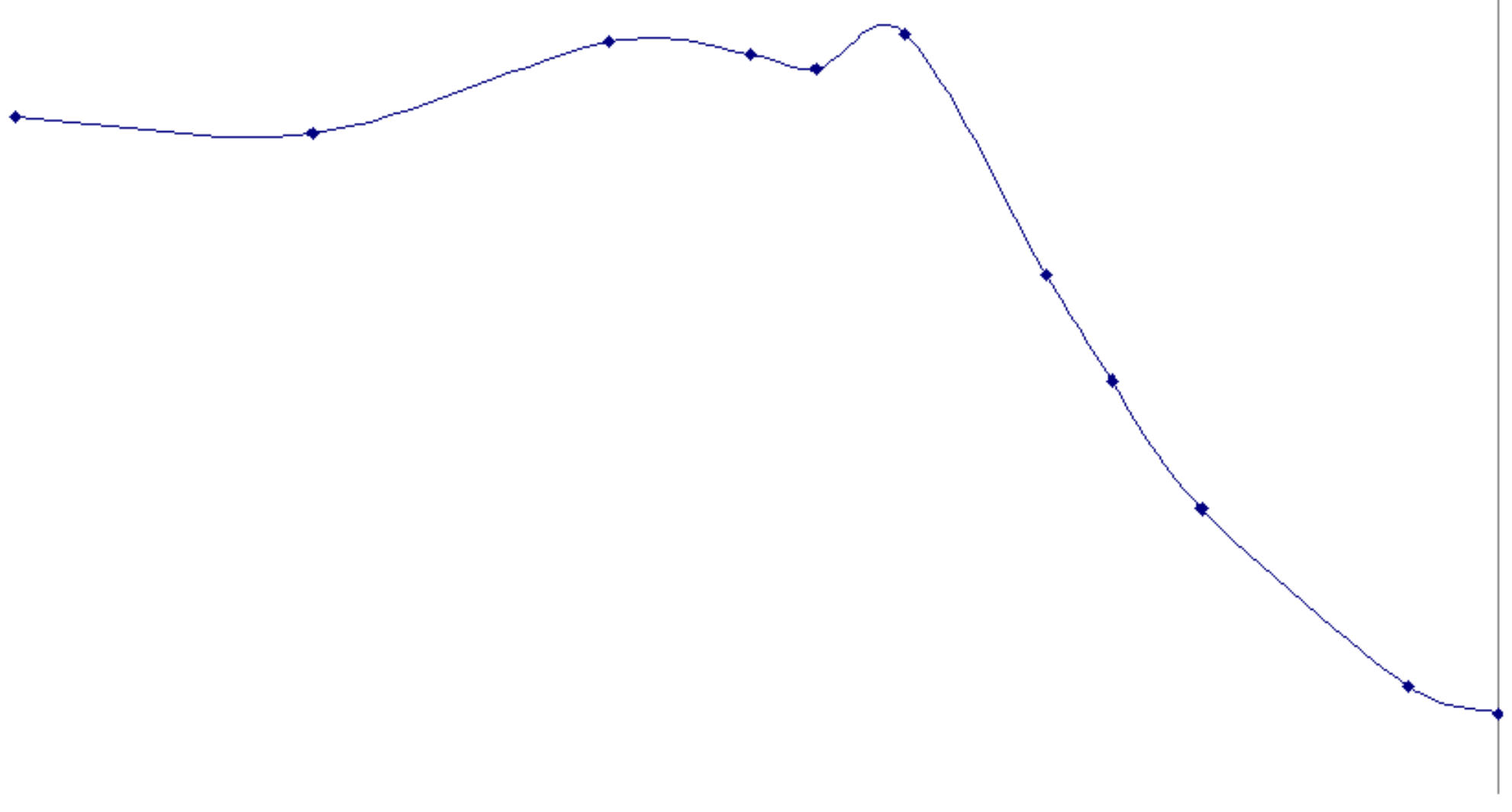




\section{Appendix J}

\section{Calibration Curves for Carnauba Wax} on Aluminum Panels and Stainless Steel Disks

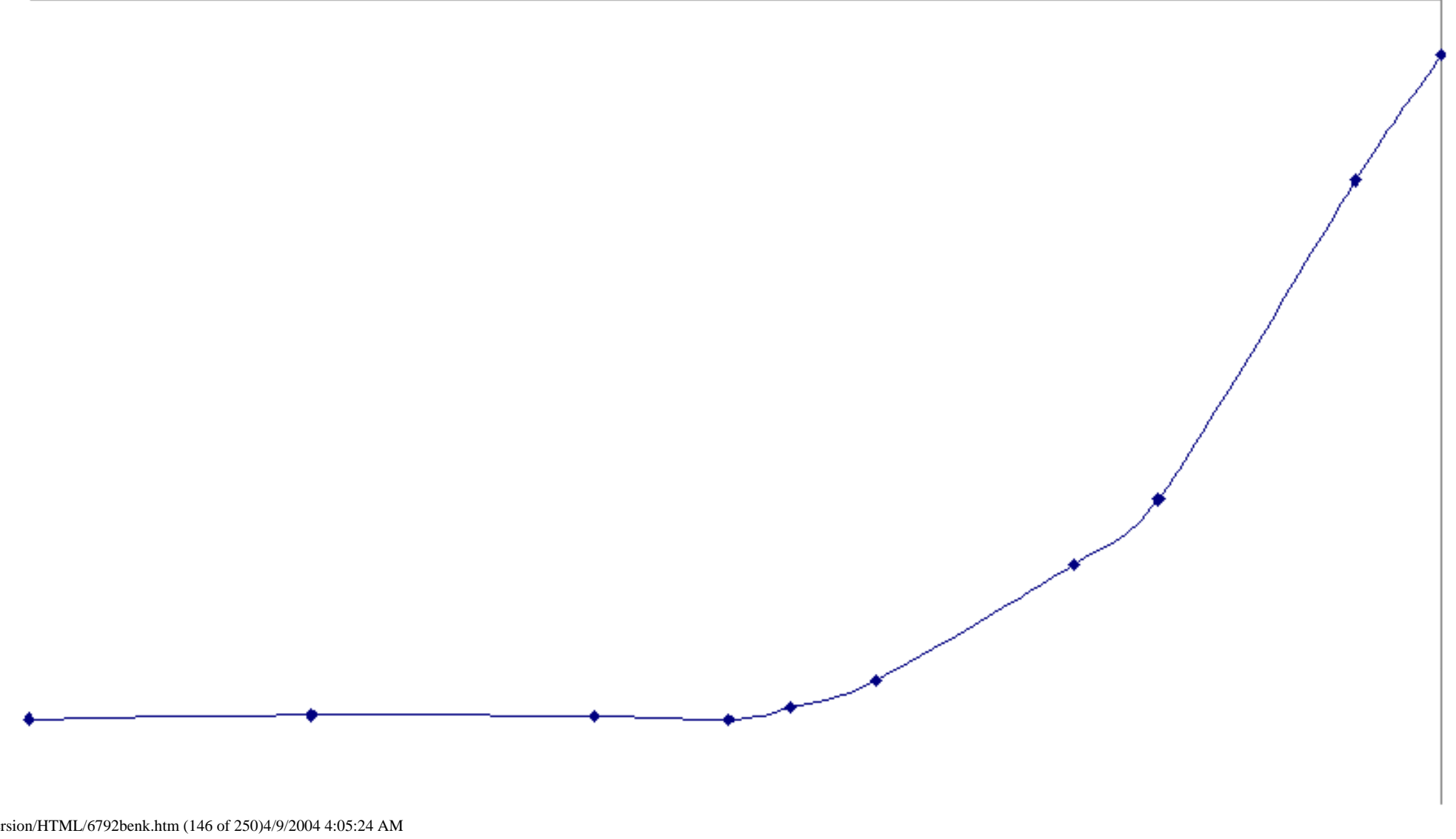




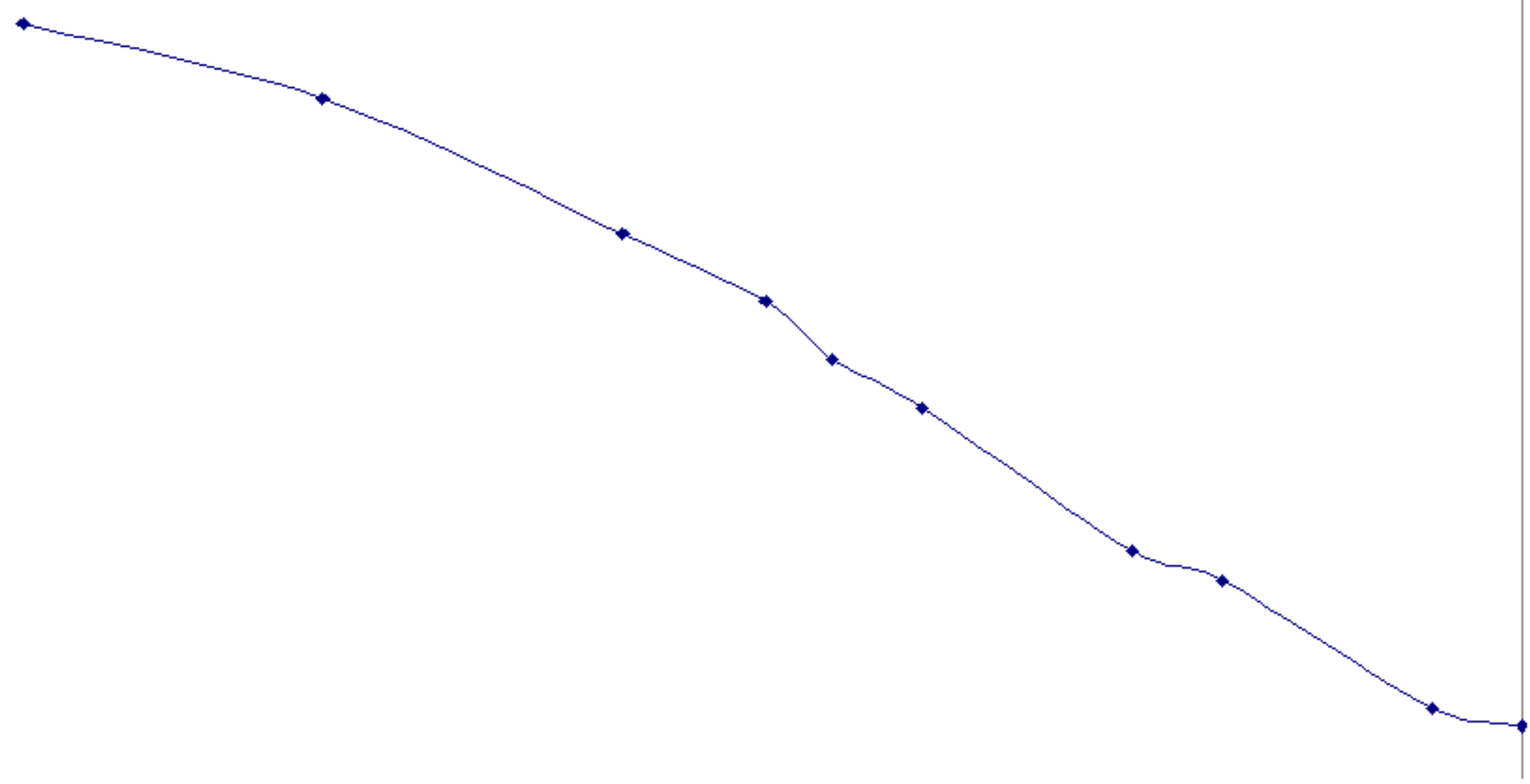




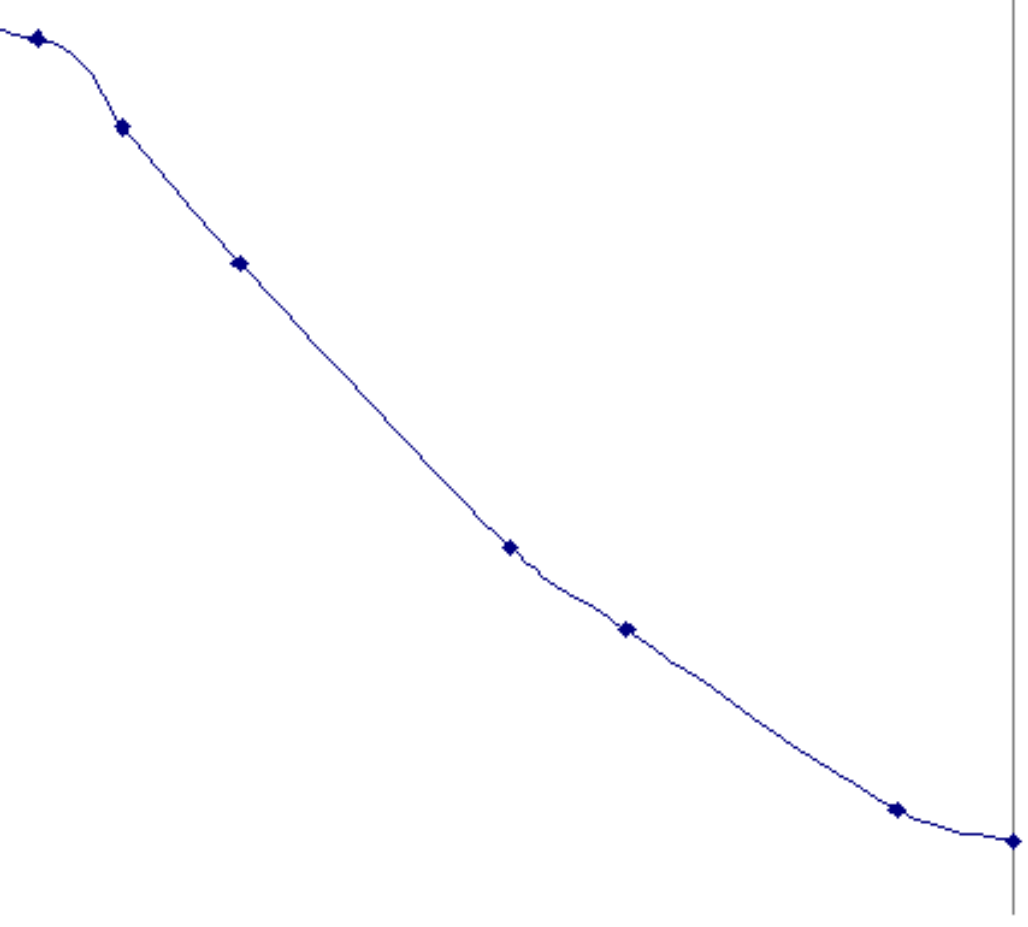



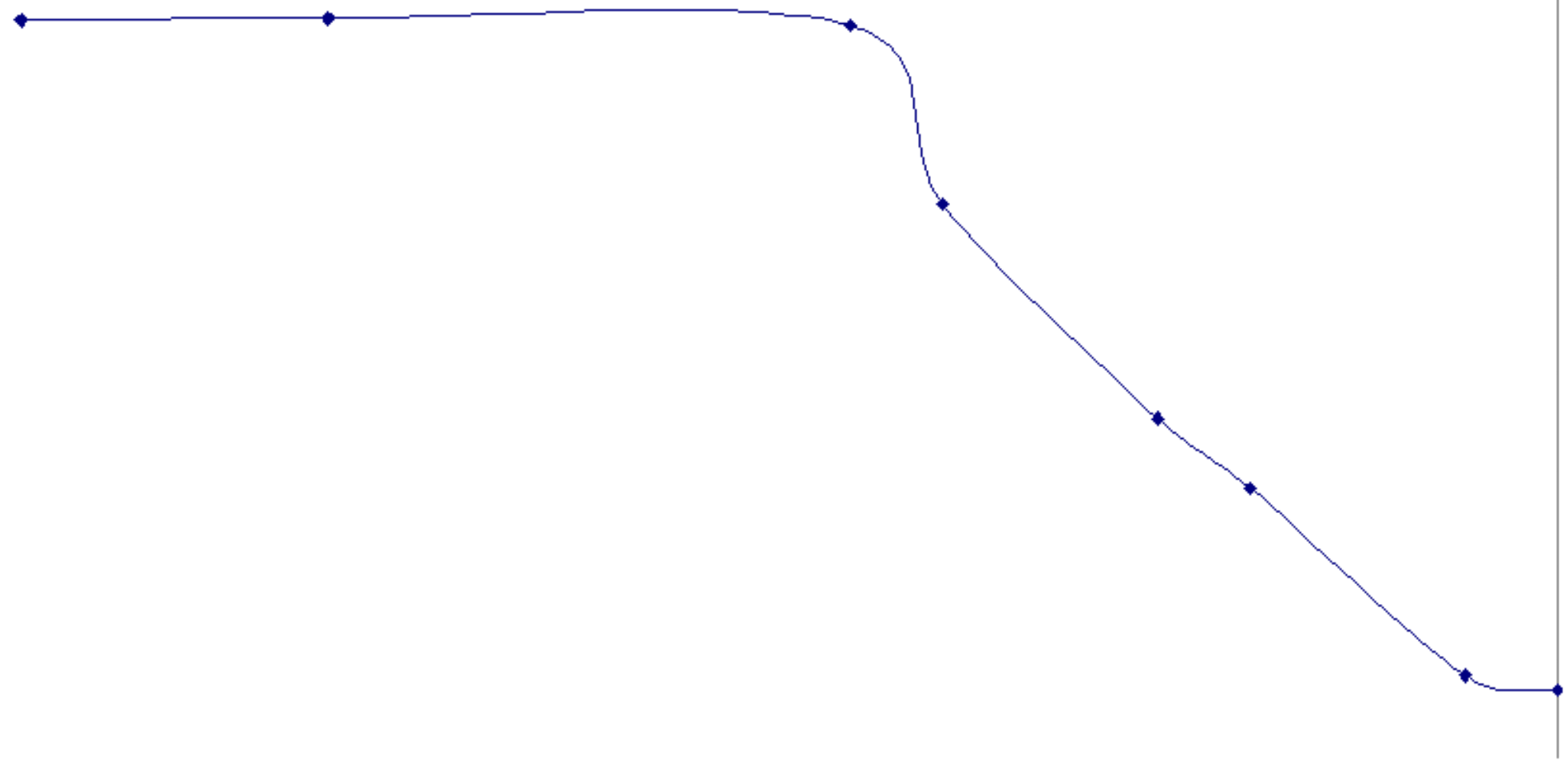


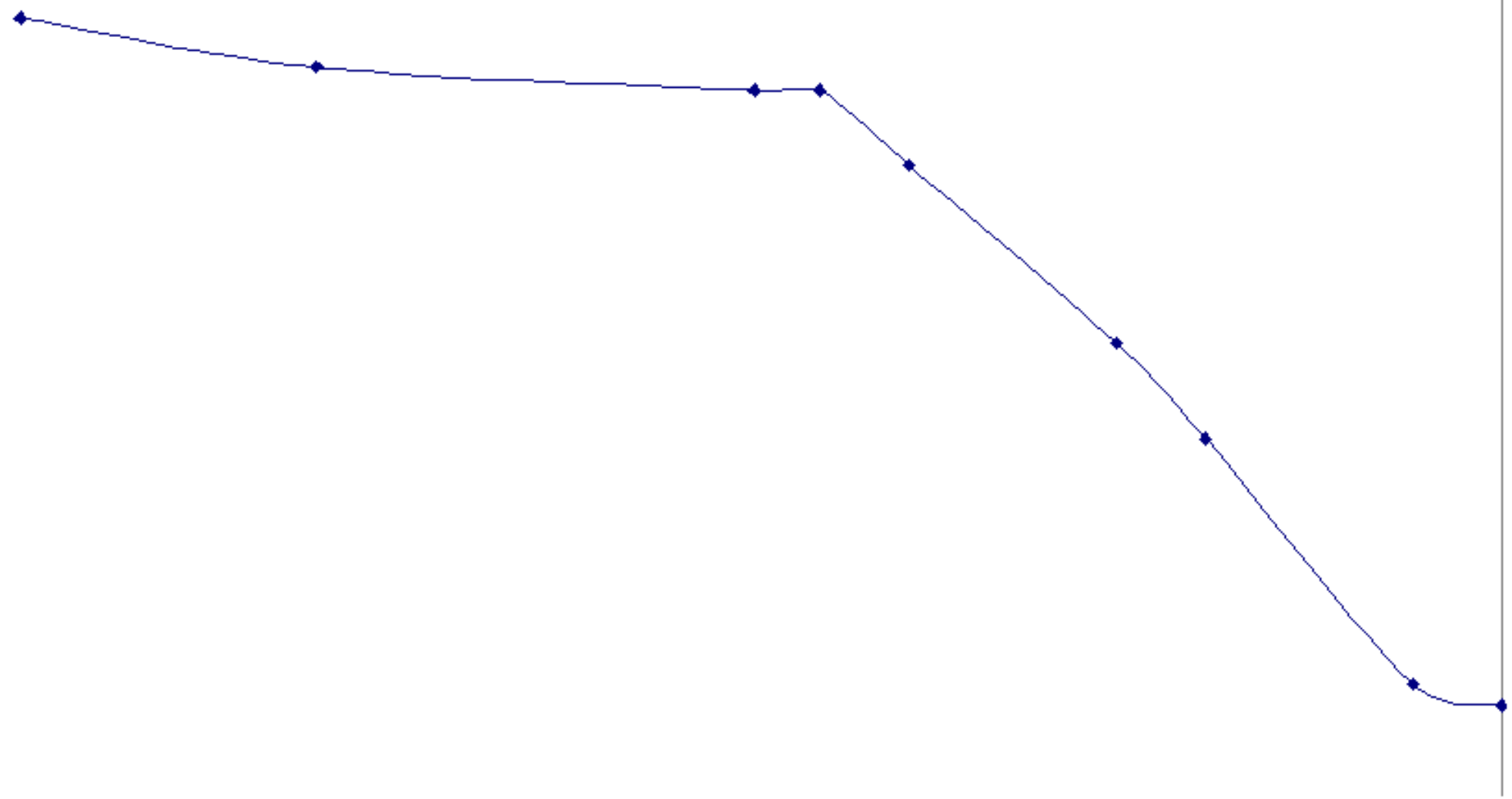




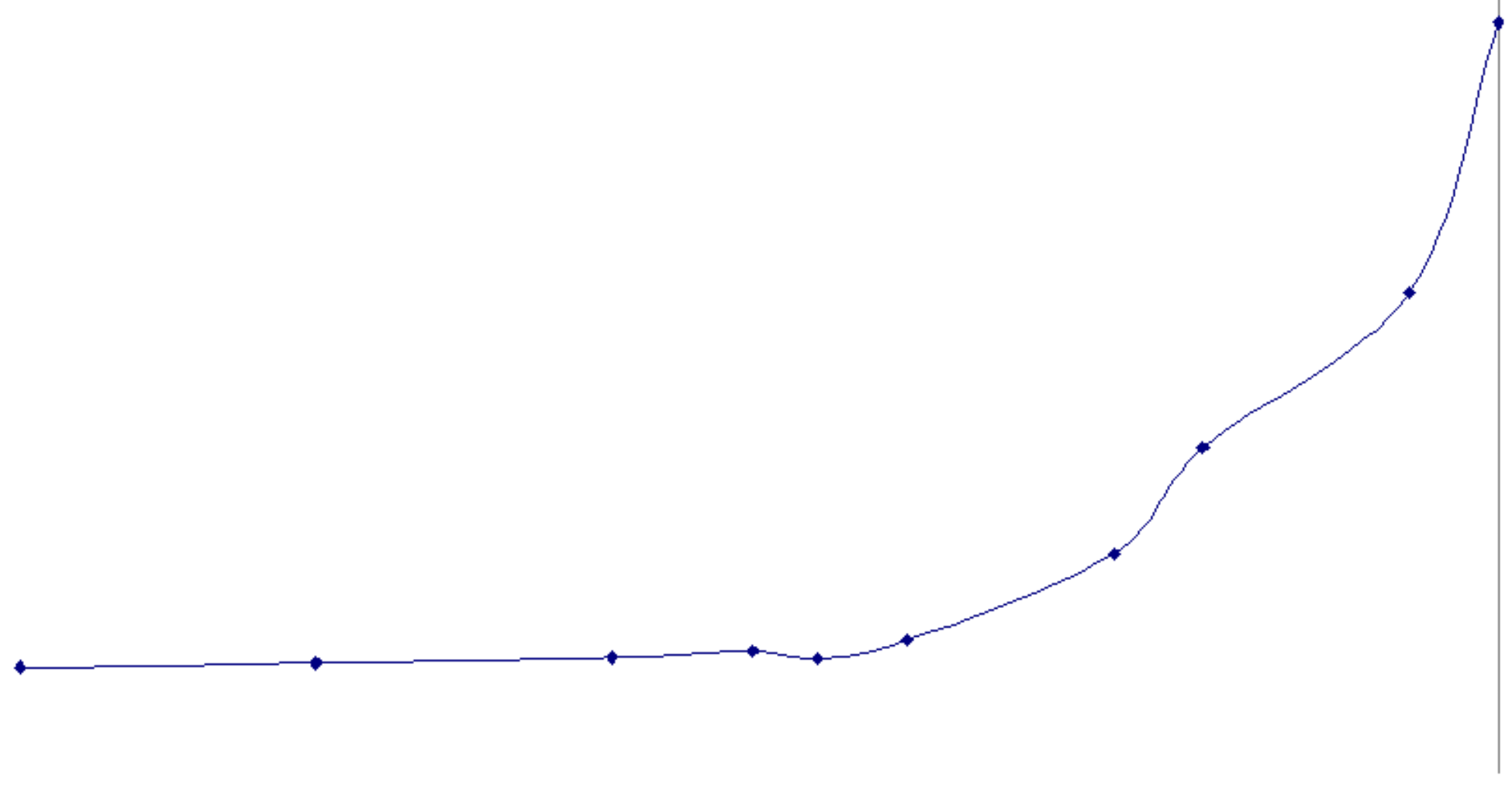




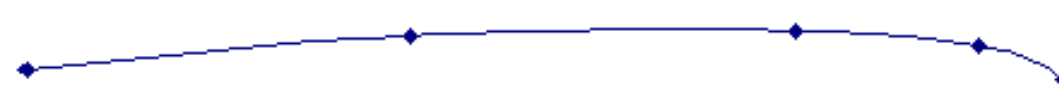




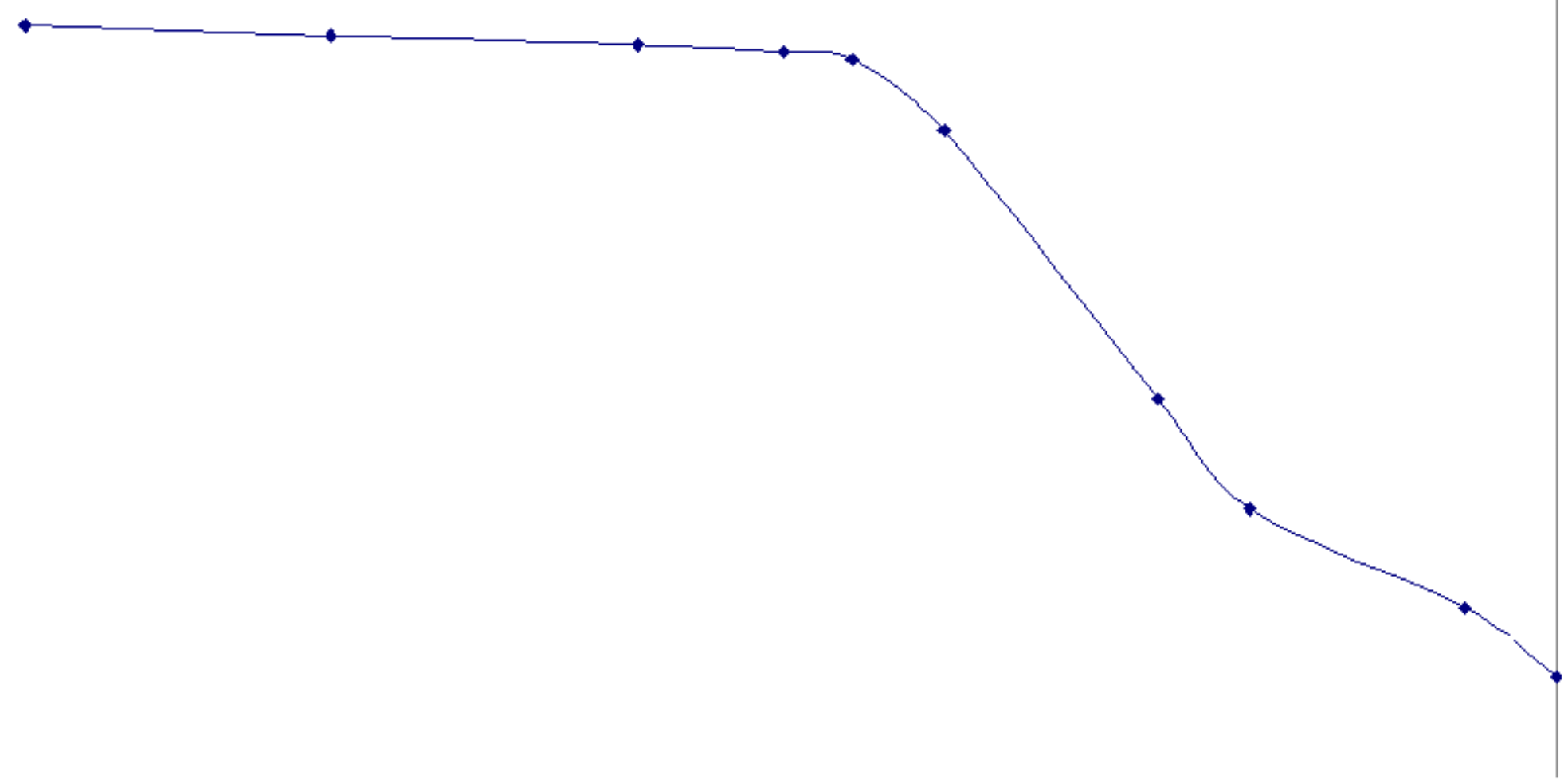




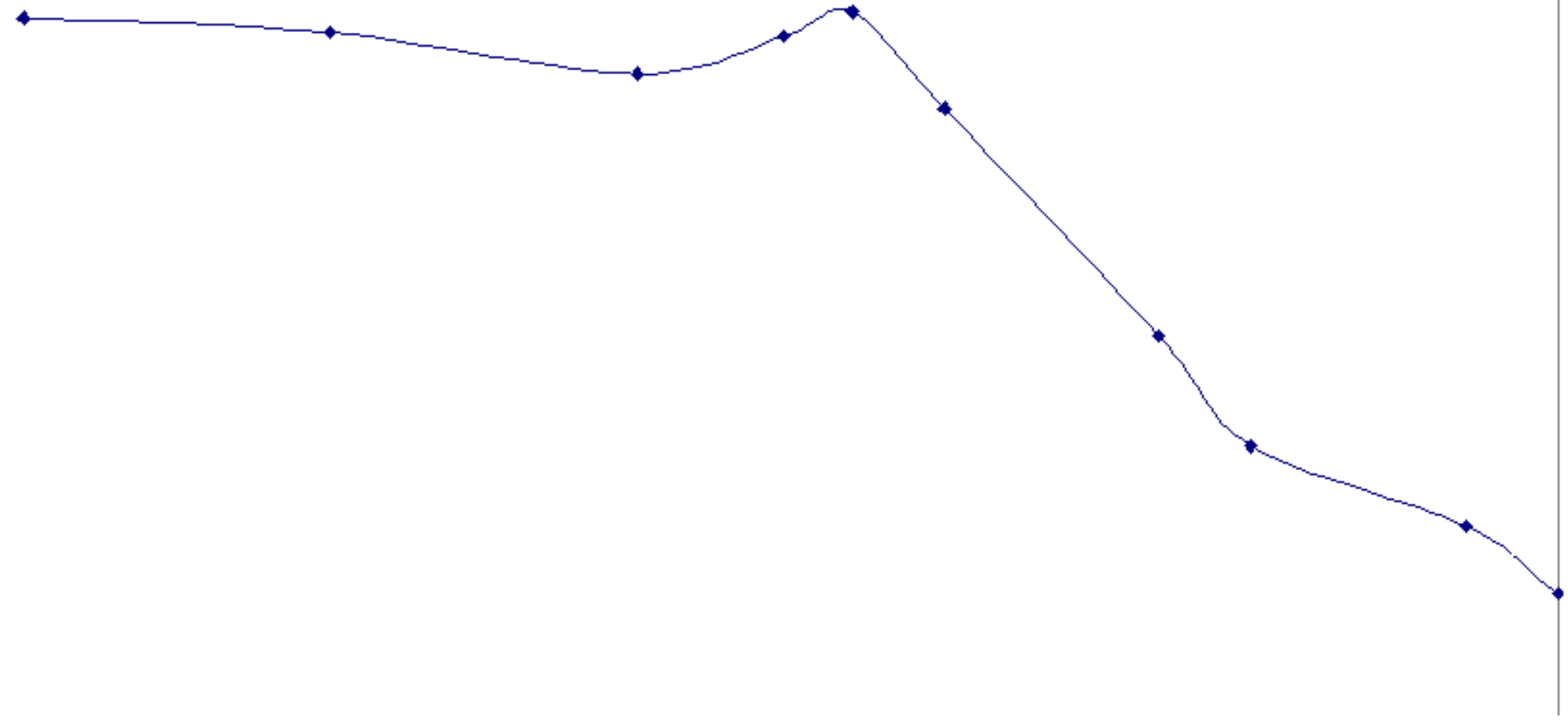


<smiles>CCCCC</smiles> 


\section{Appendix K}

\section{Calibration Curves for OSR Mold Release} on Aluminum Panels and Stainless Steel Disks

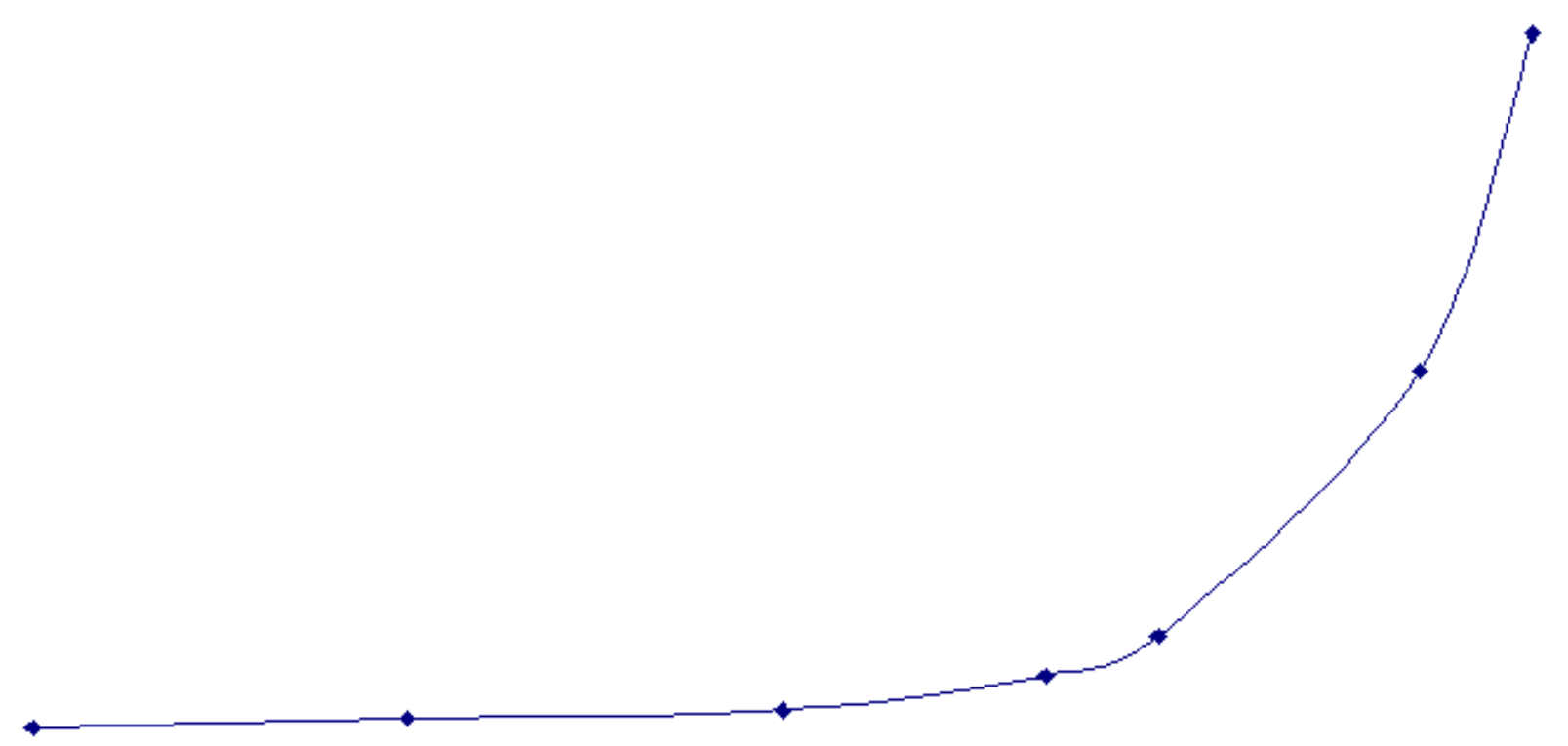


FY98 Annual Report

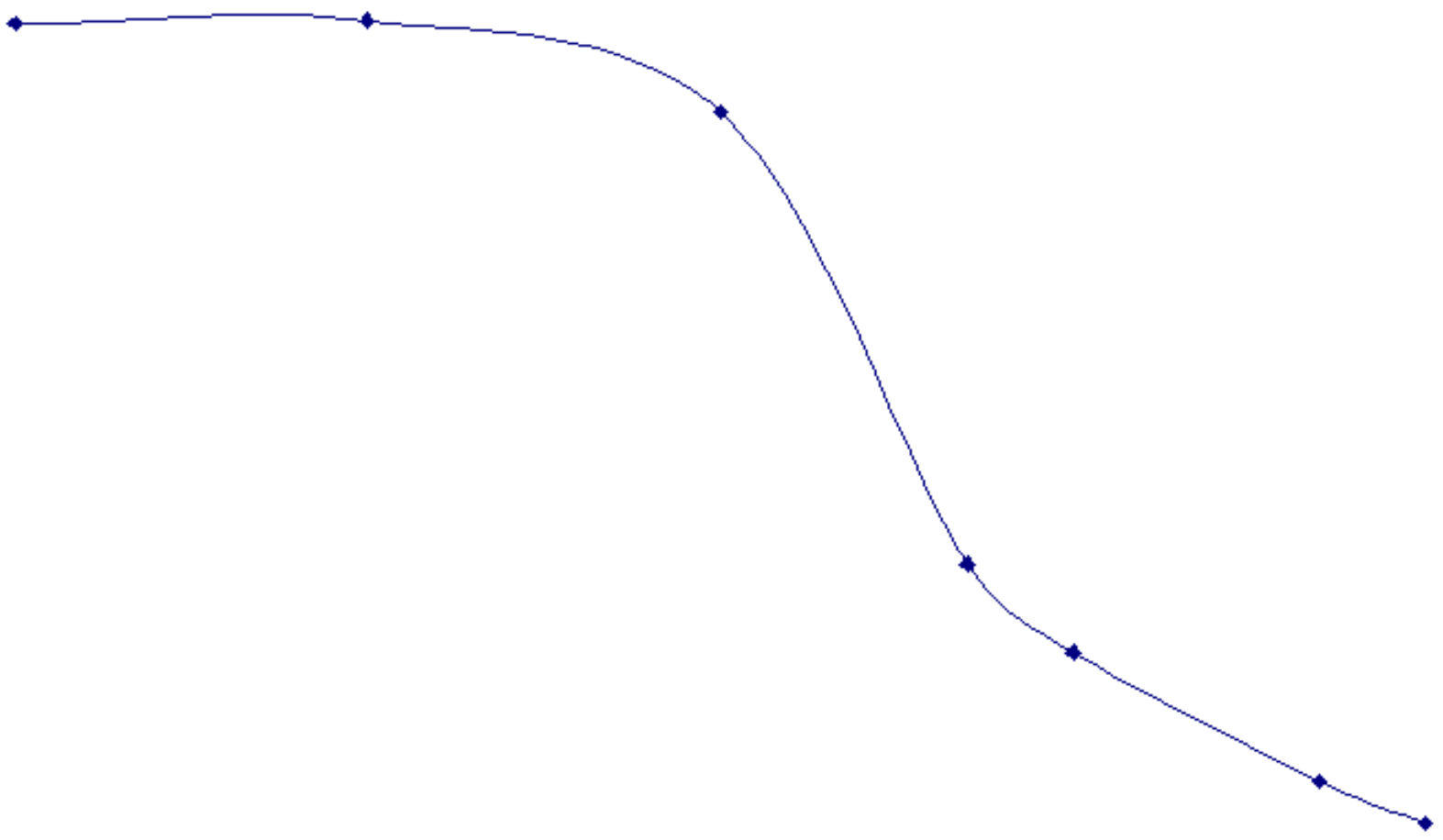

file://C//ManualConversion/HTML/6792benk.htm (157 of 250)4/9/2004 4:05:24 AM 


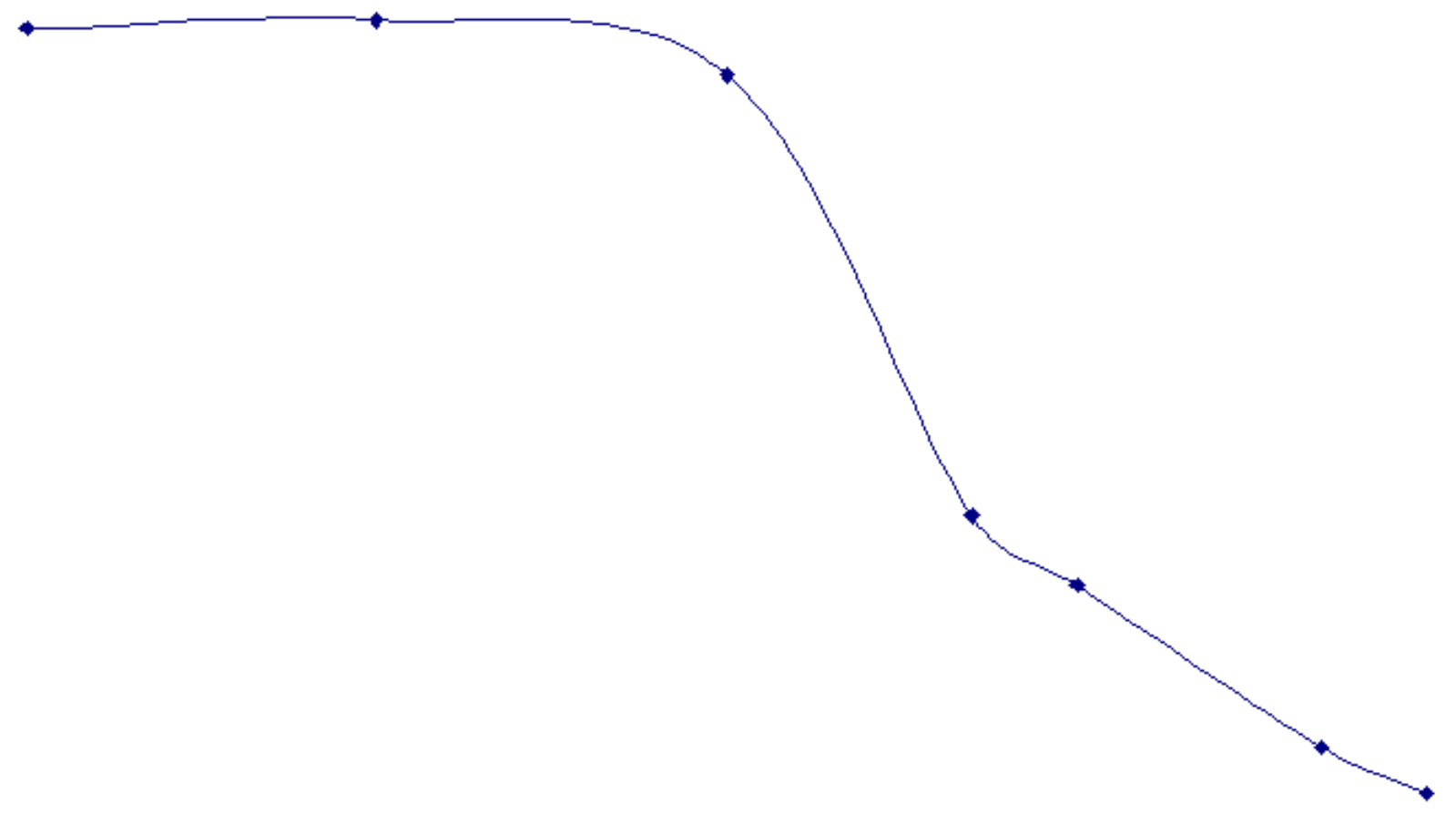




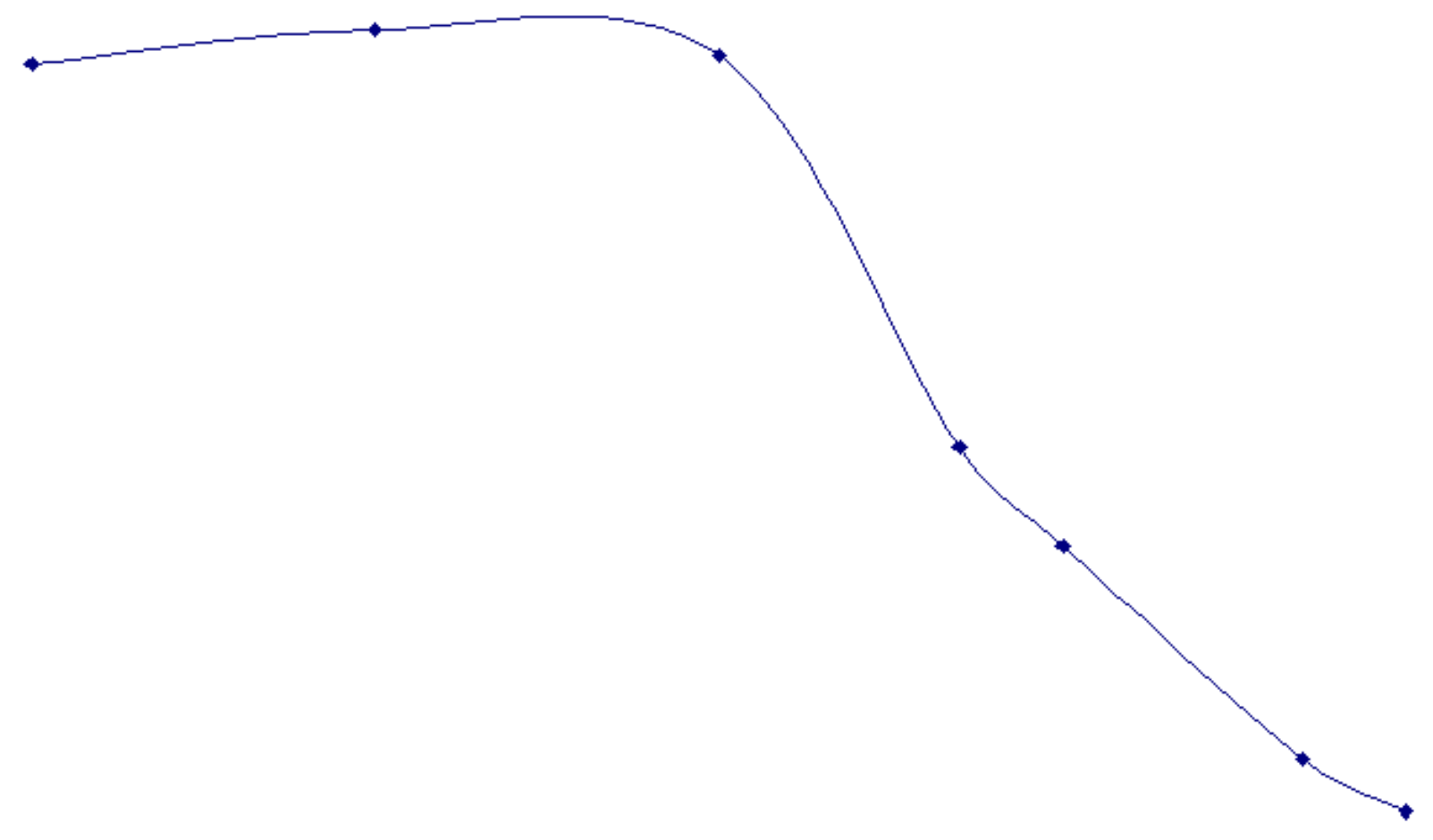




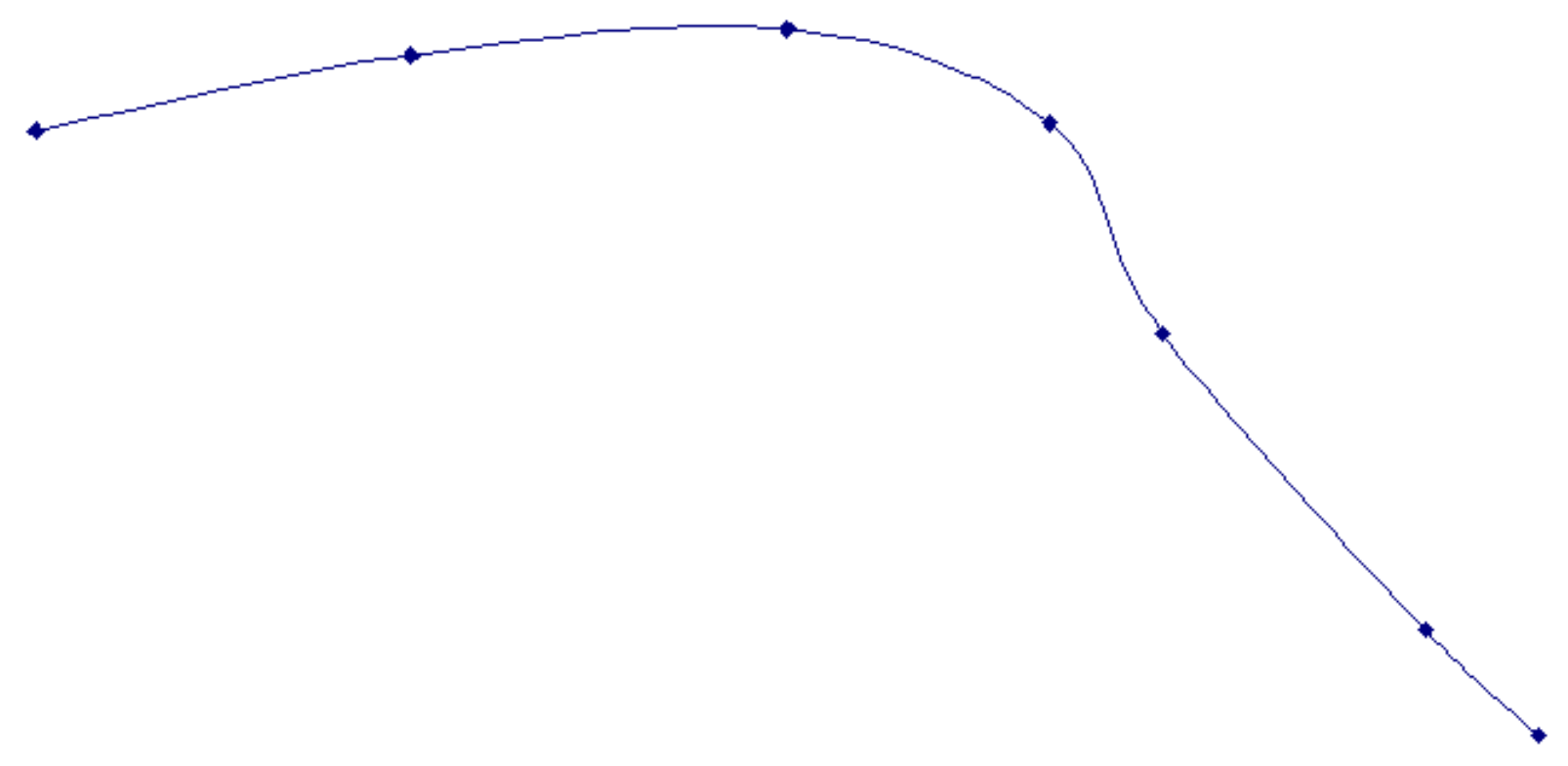


<smiles>CCC</smiles> 

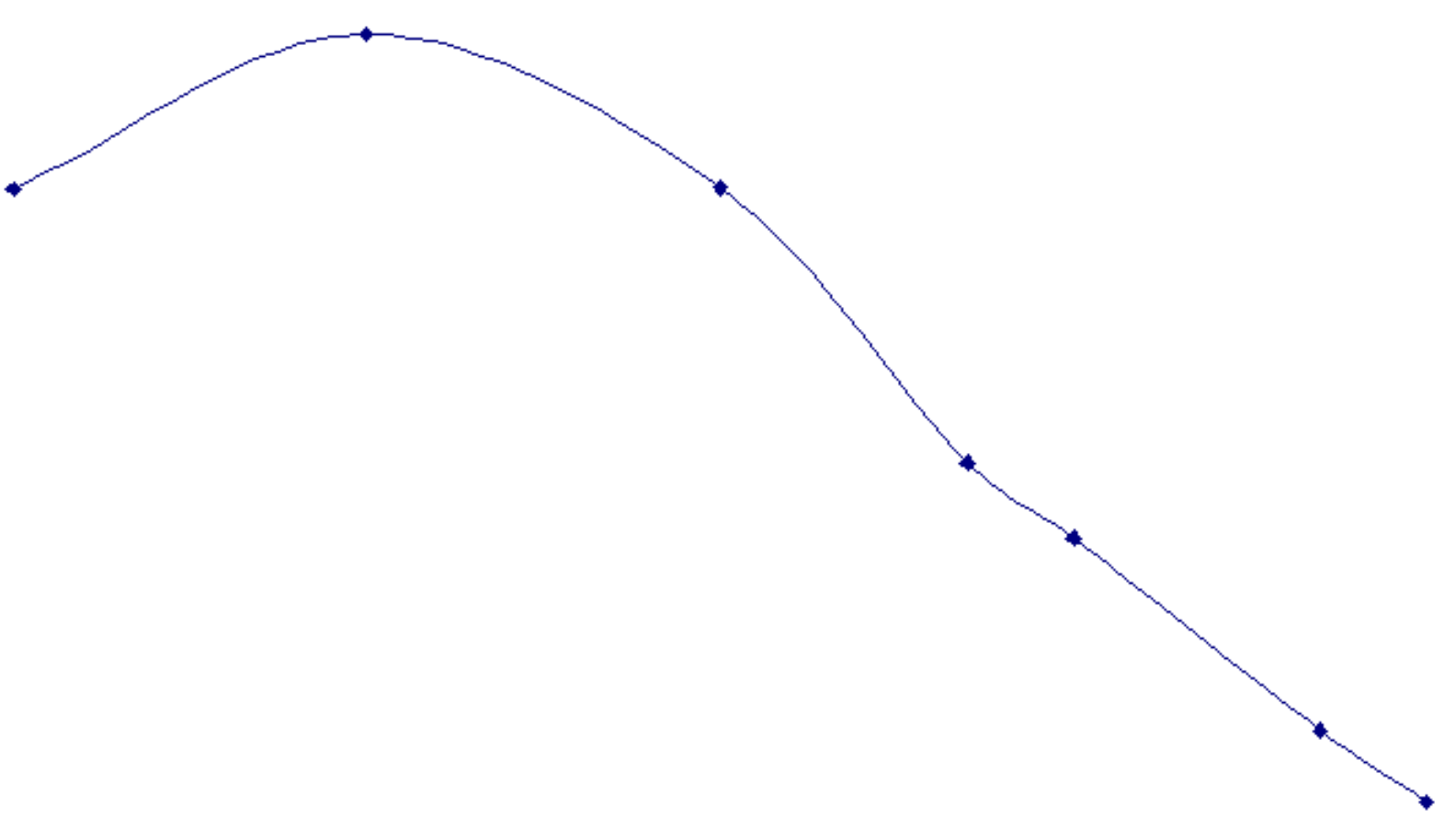
<smiles>CCCCC</smiles> 

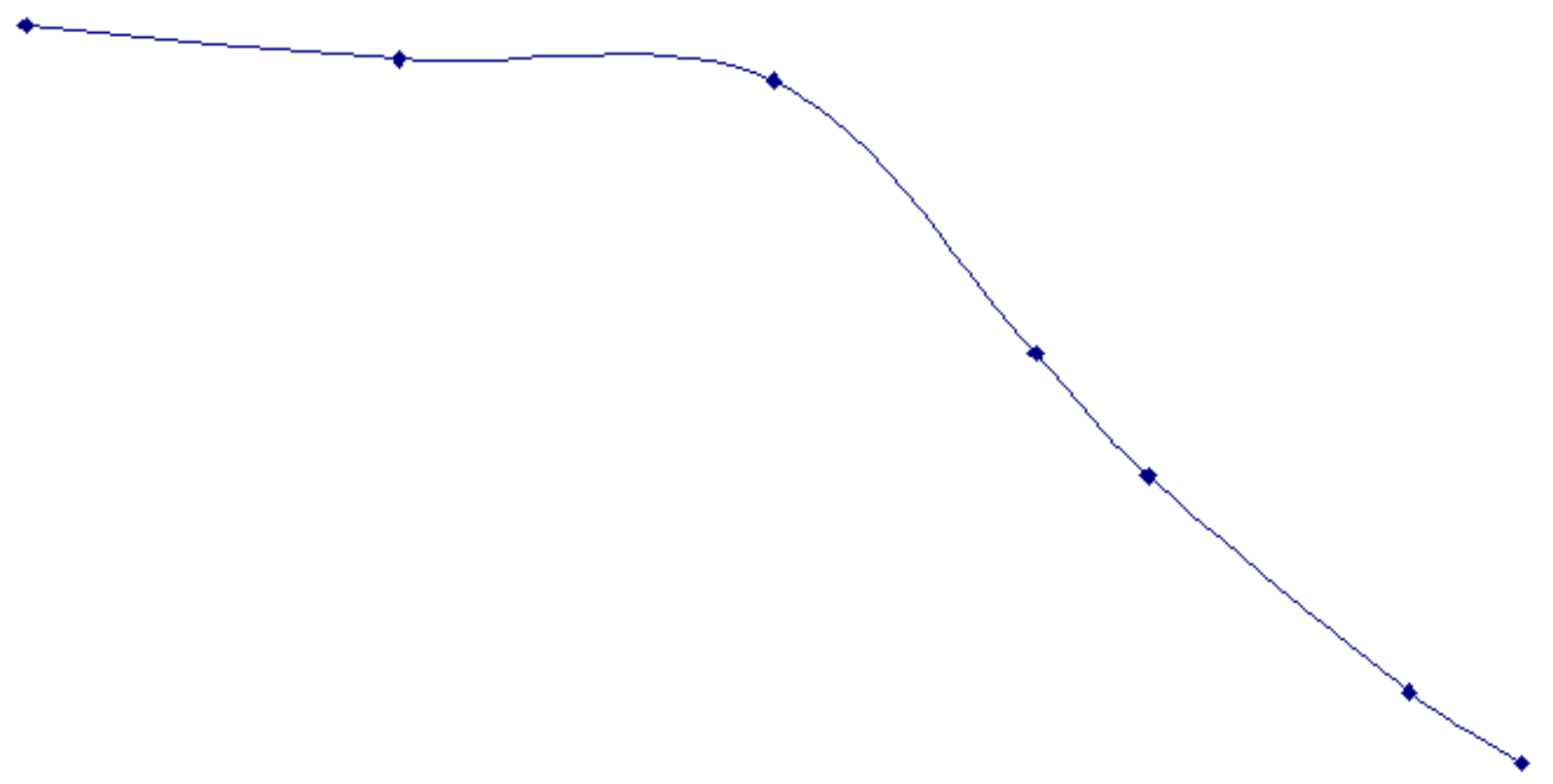


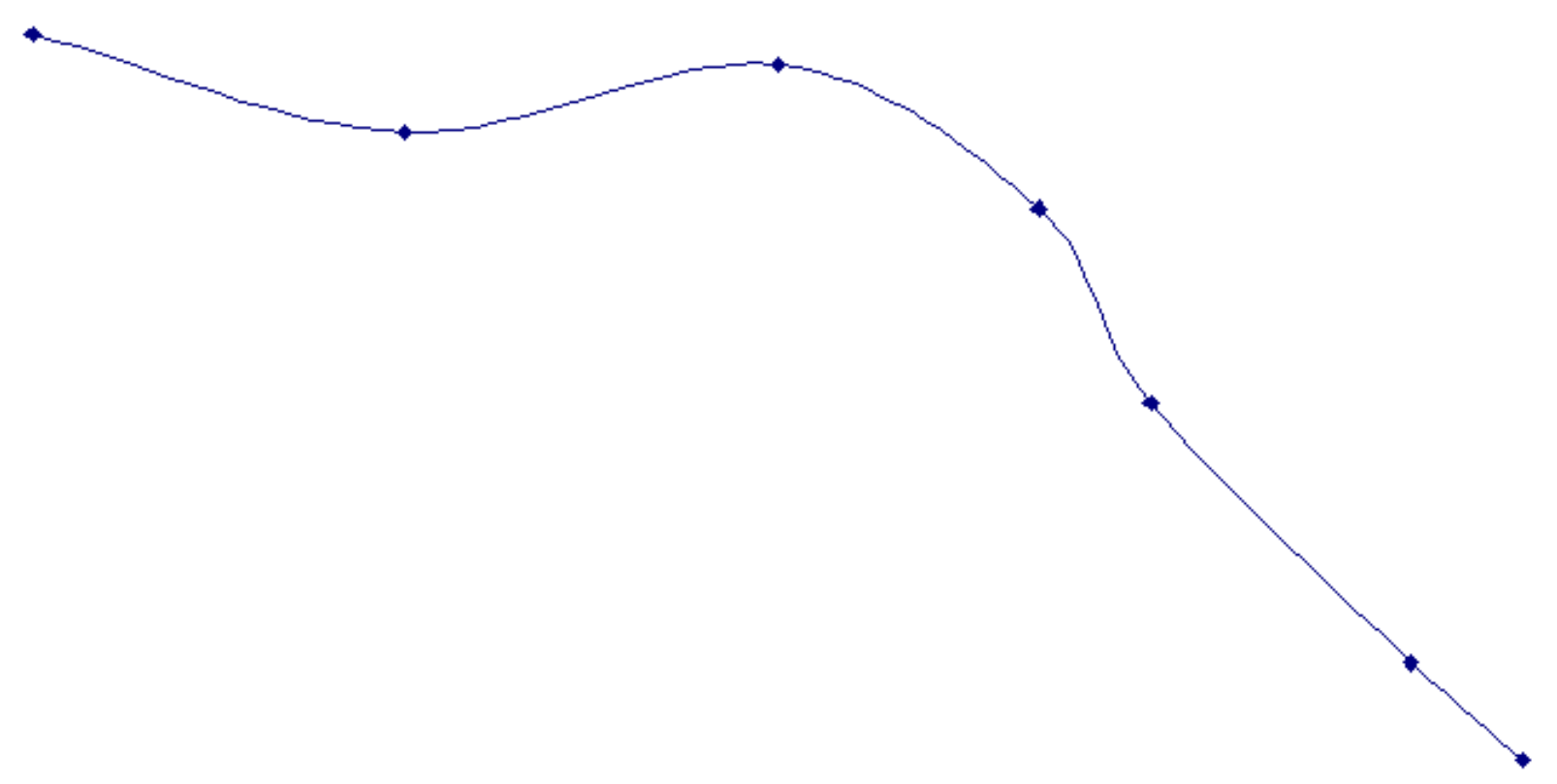




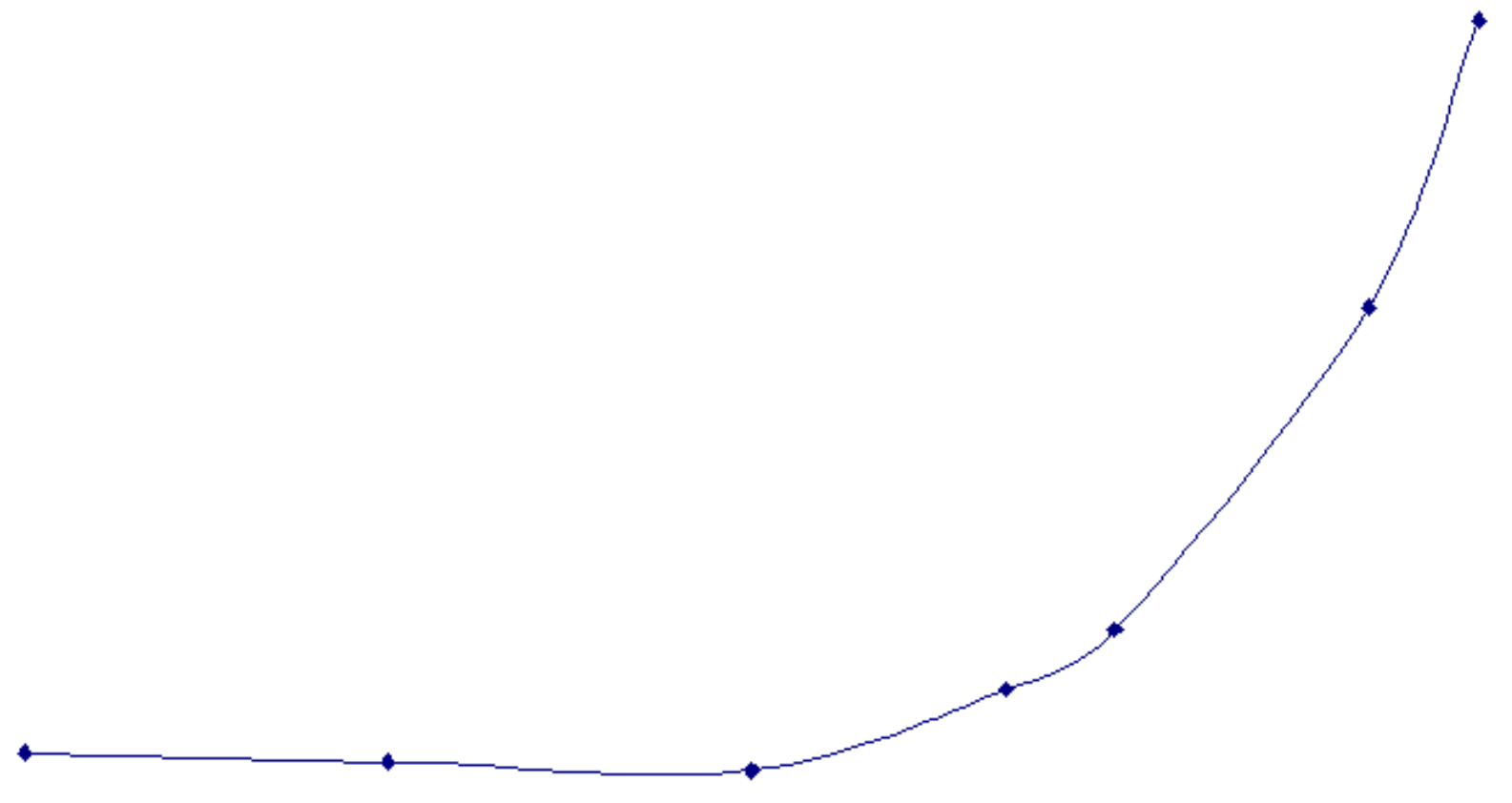


<smiles>C#CCCC</smiles> 


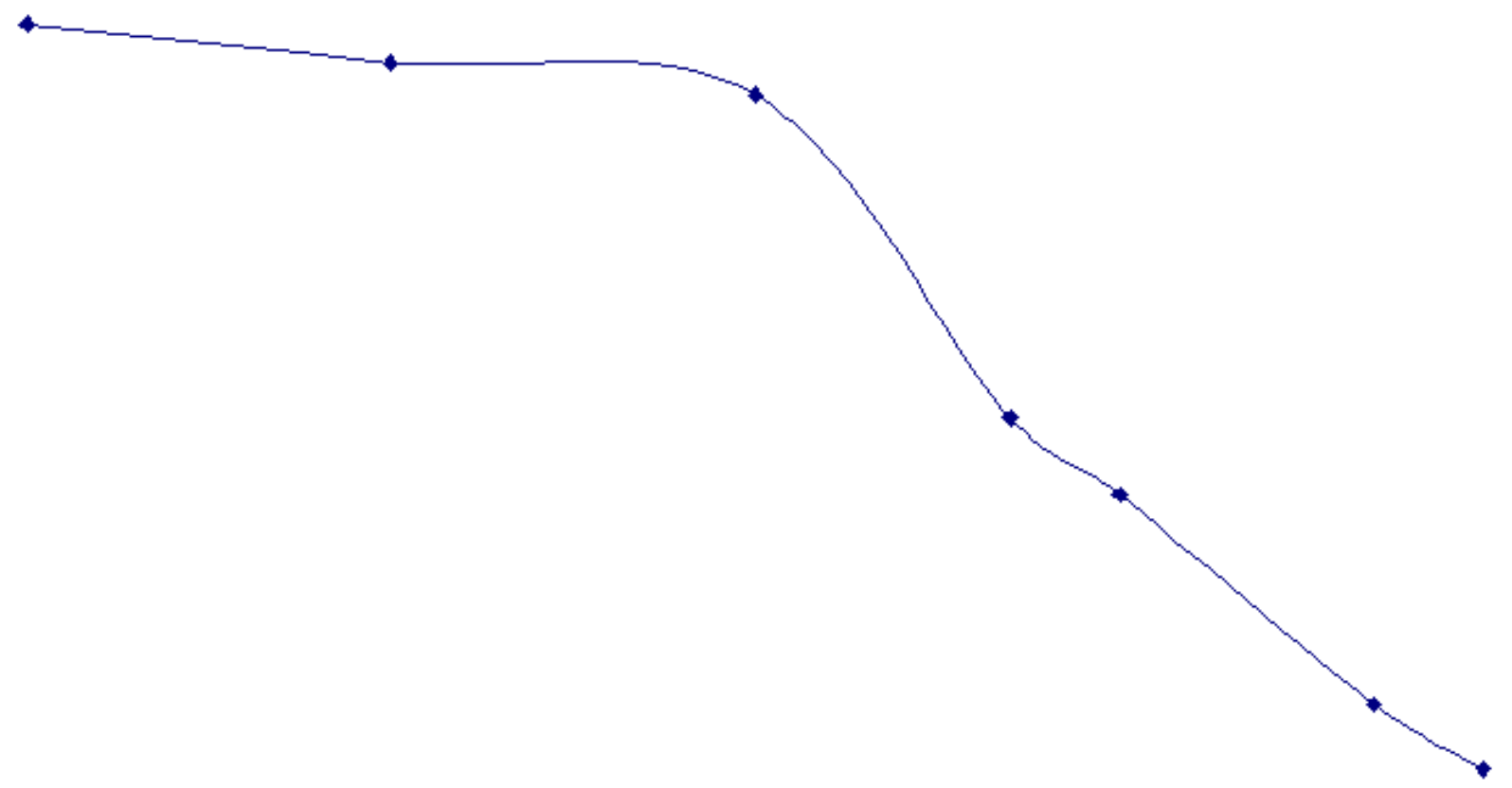




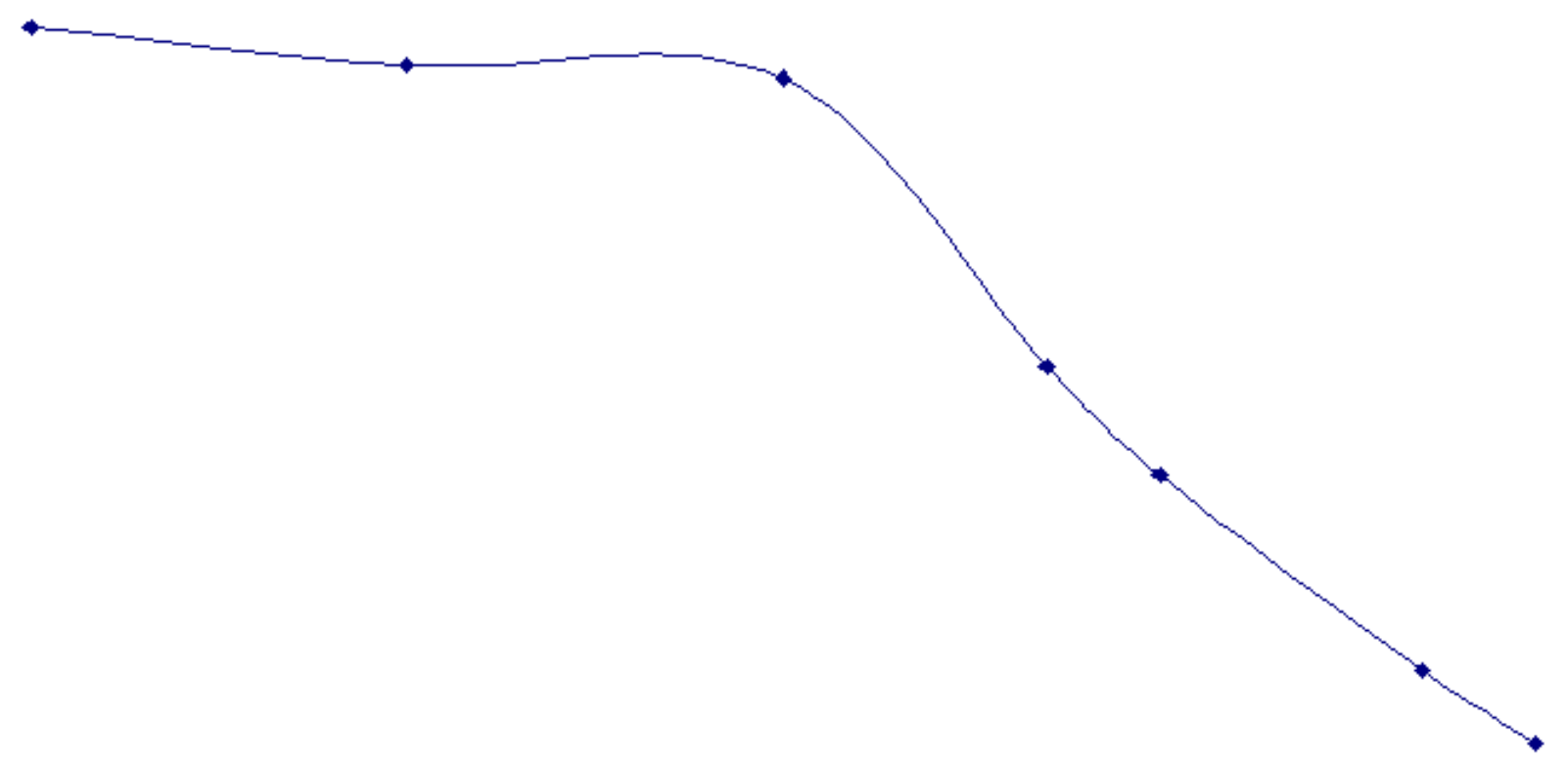




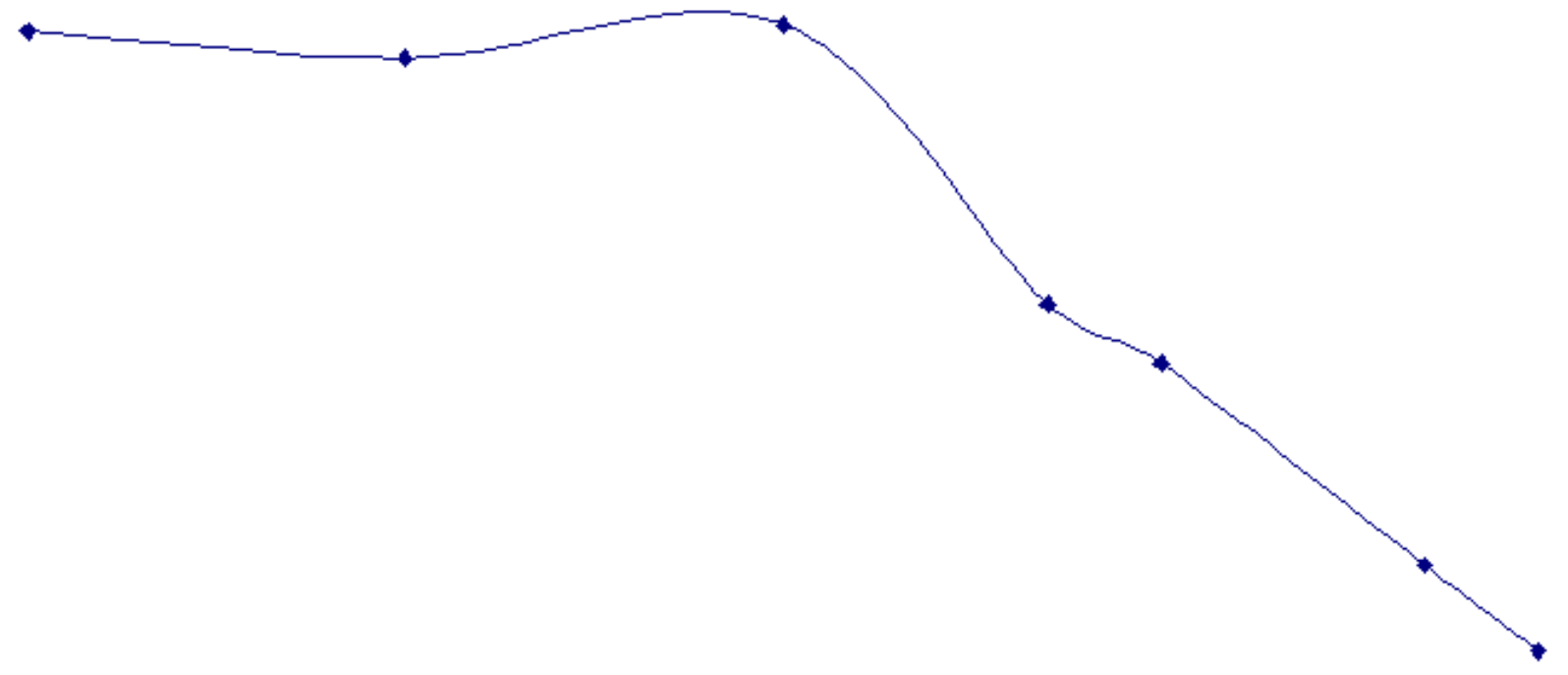


Appendix L

Calibration Curves for Thalco 550 Mold Release

on Aluminum Panels and Stainless Steel Disks

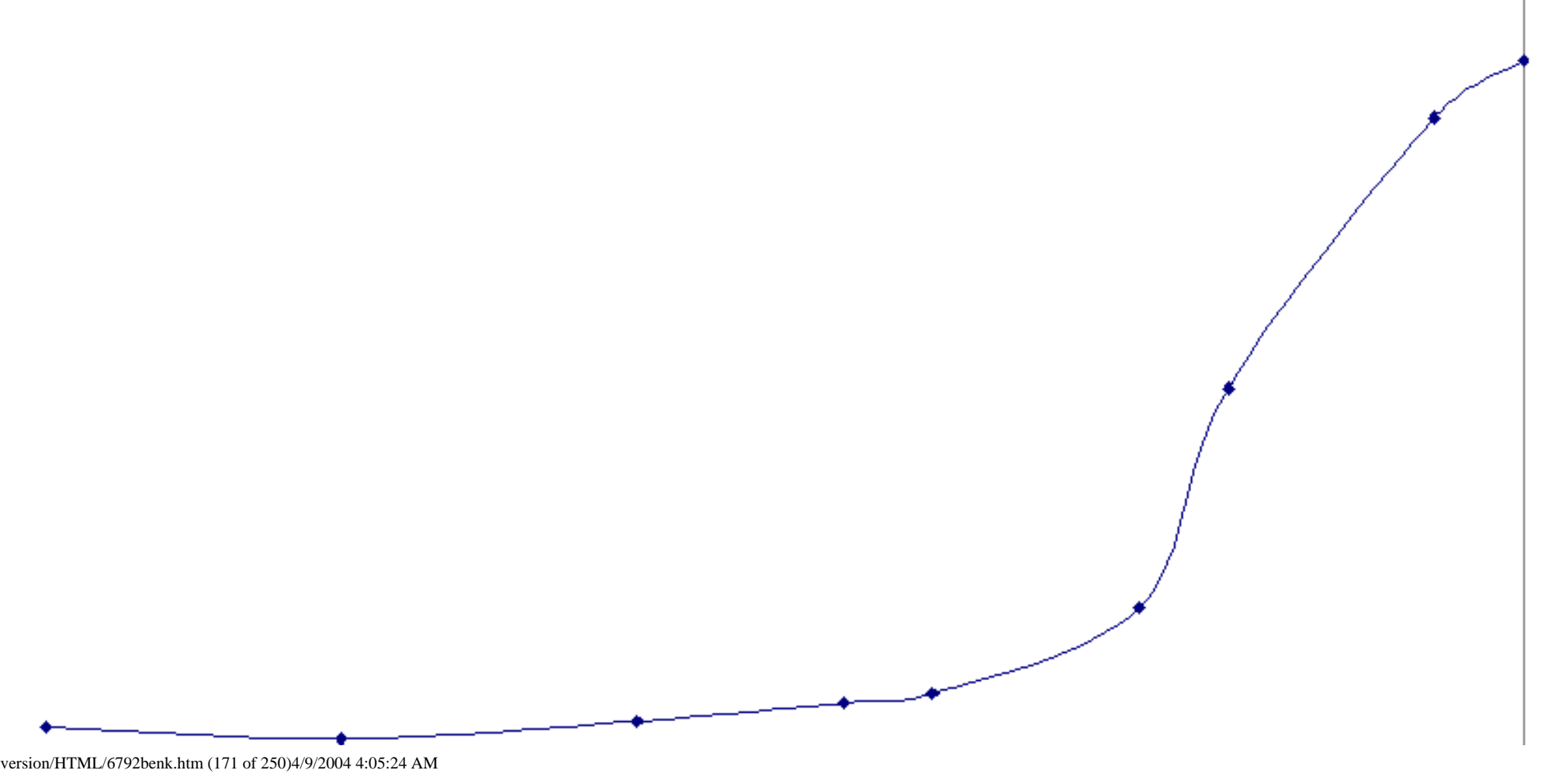

file:///C/ManualConversion/HTML/6792benk.htm (171 of 250)4/9/2004 4:05:24 AM 


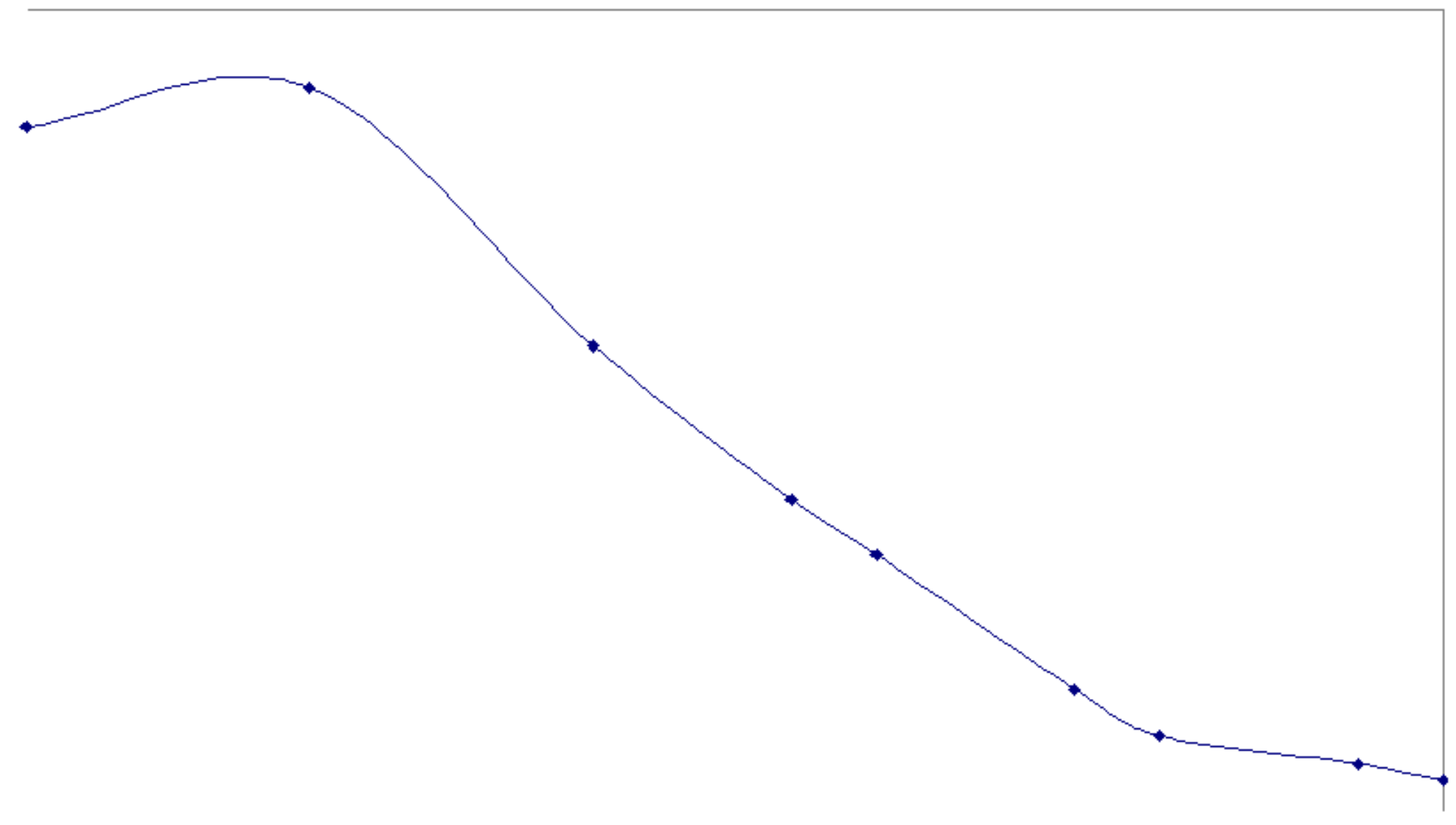




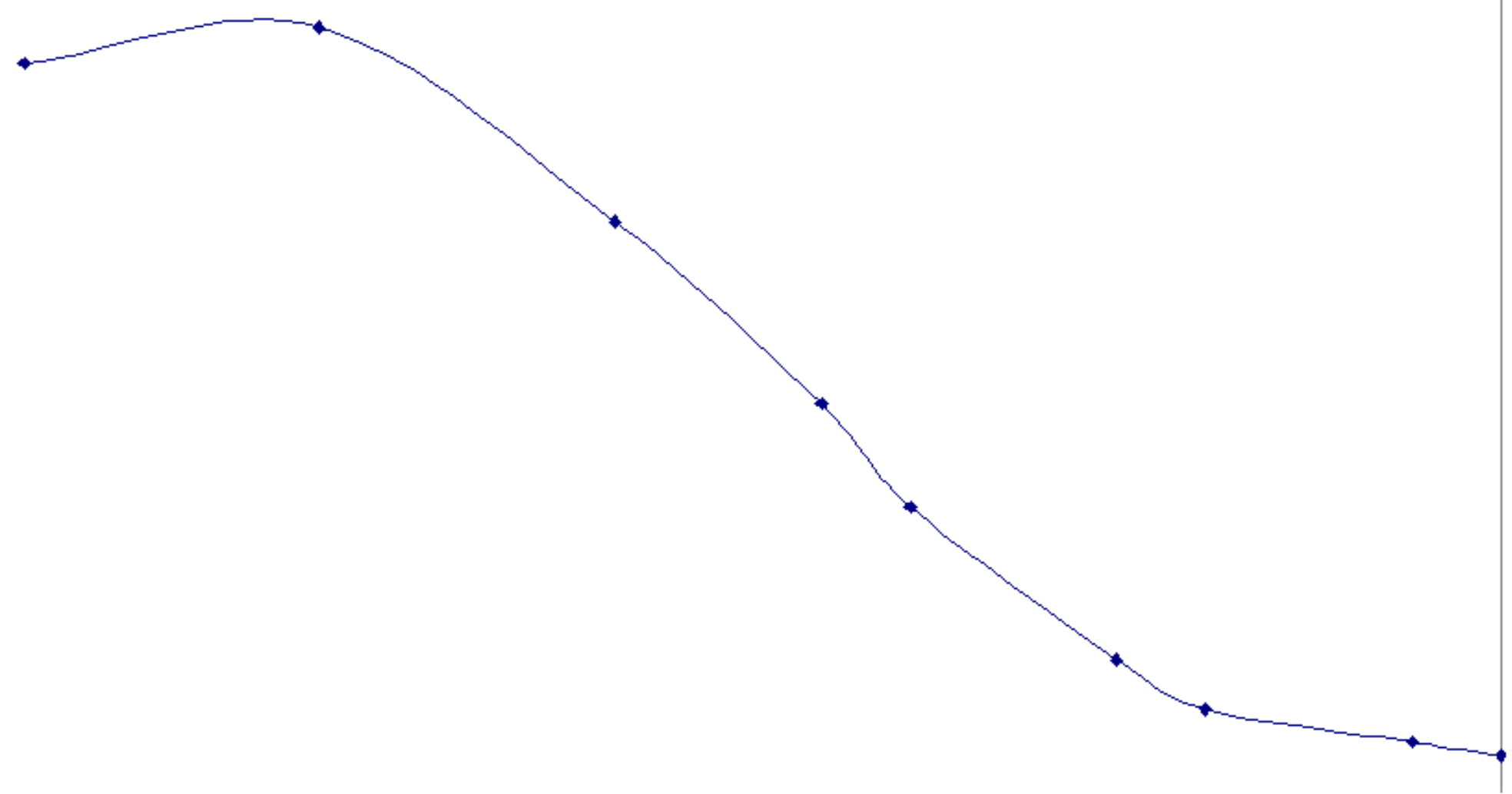



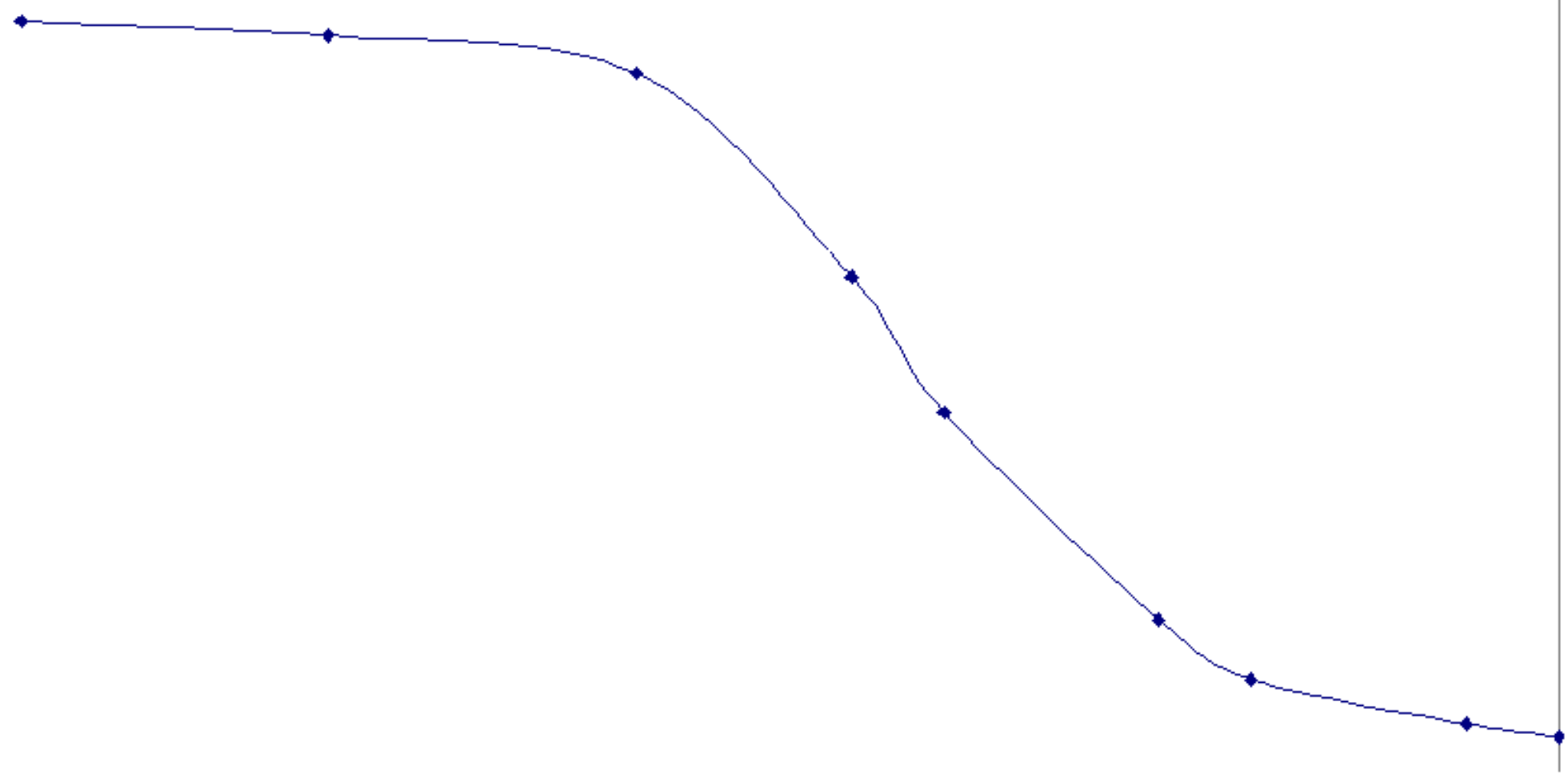


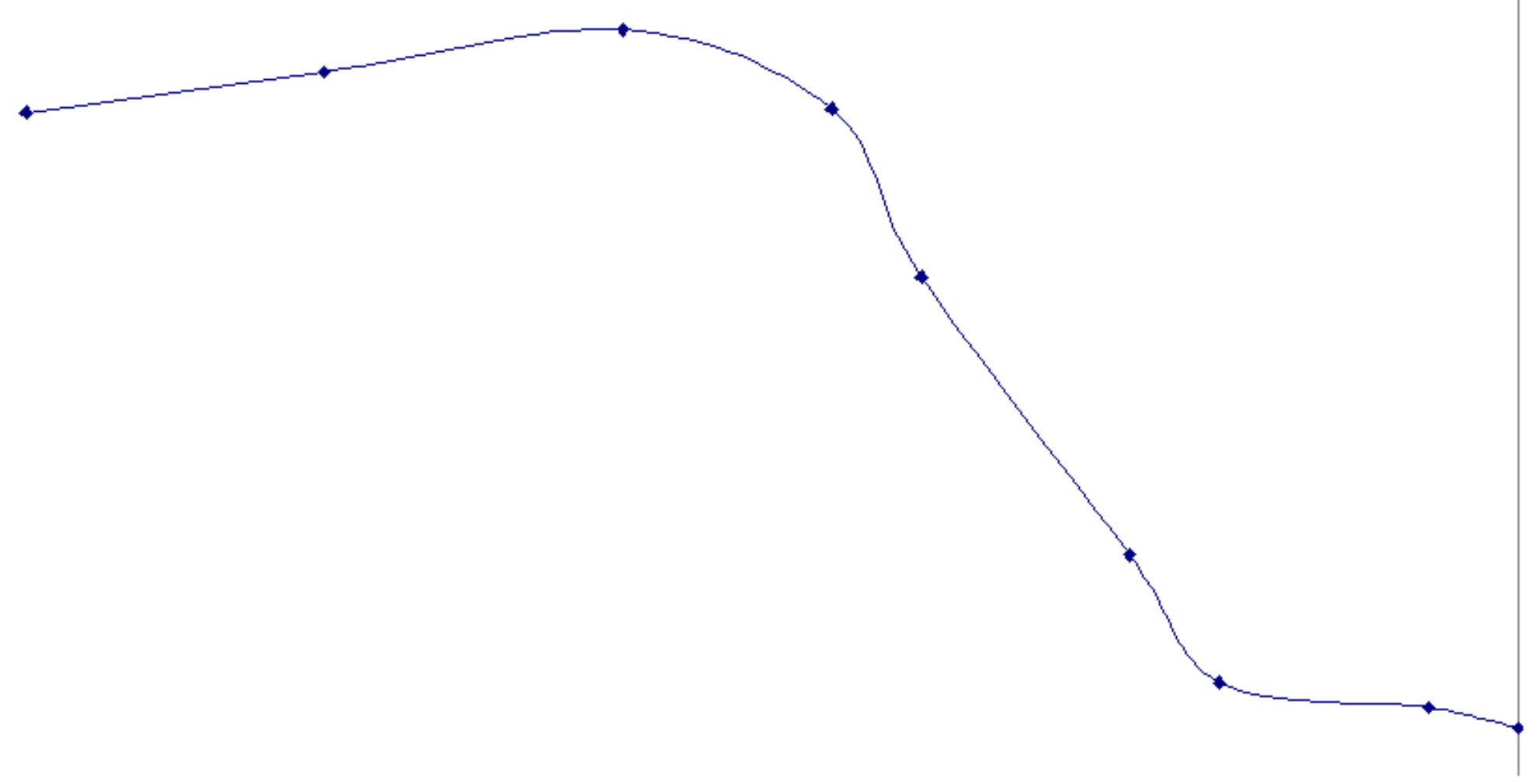


FY98 Annual Report

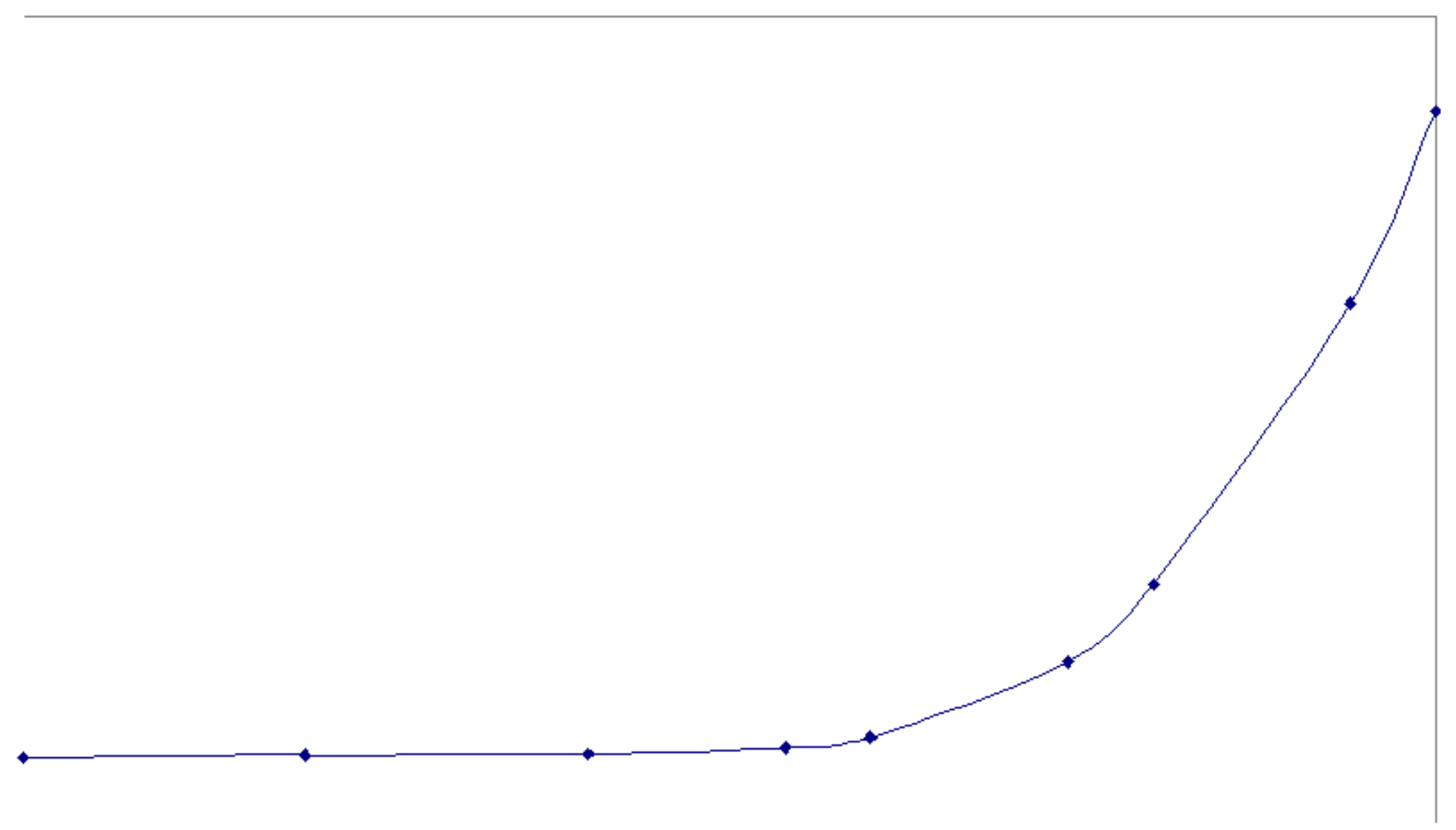

file:///C//ManualConversion/HTML/6792benk.htm (176 of 250)4/9/2004 4:05:24 AM 
4
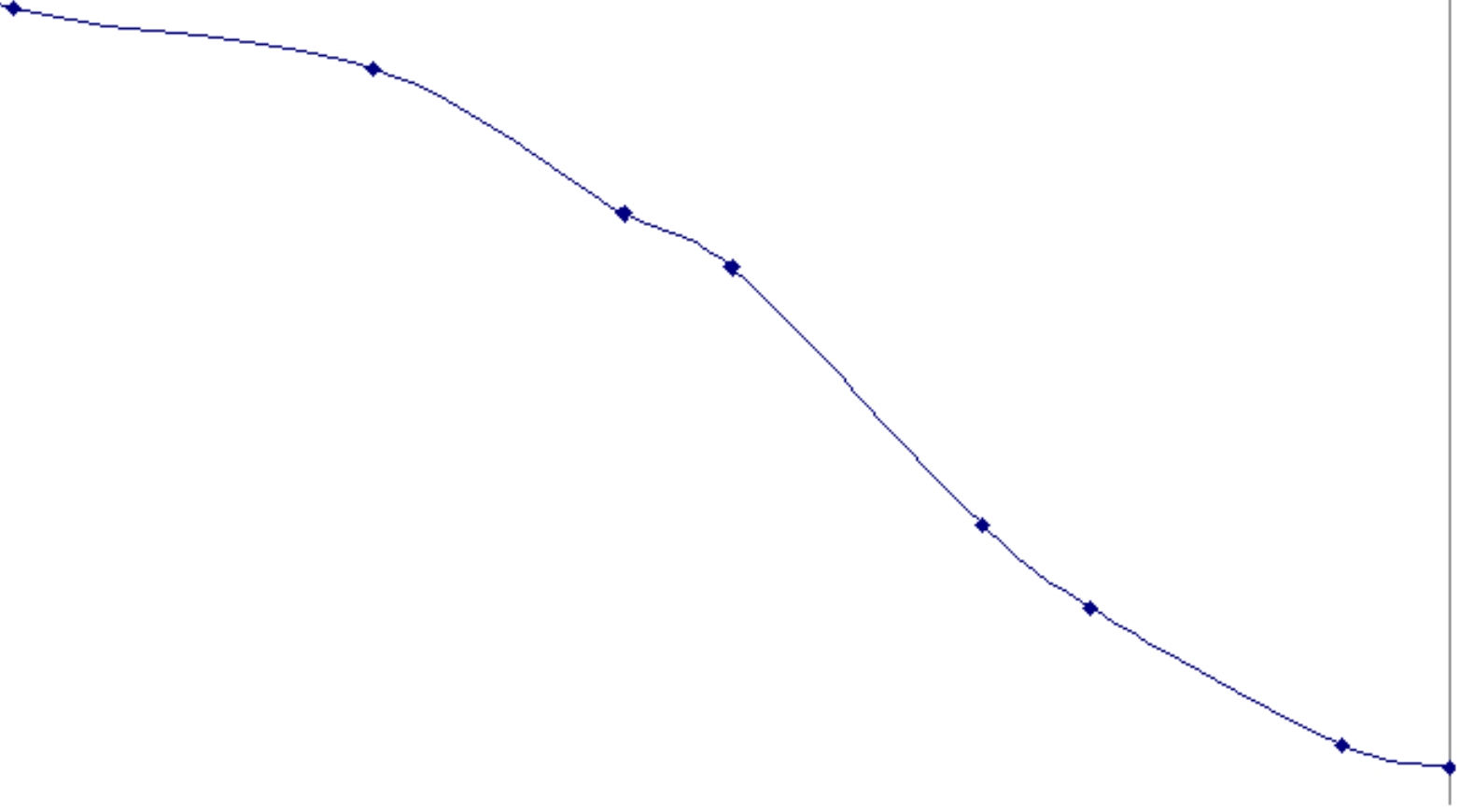


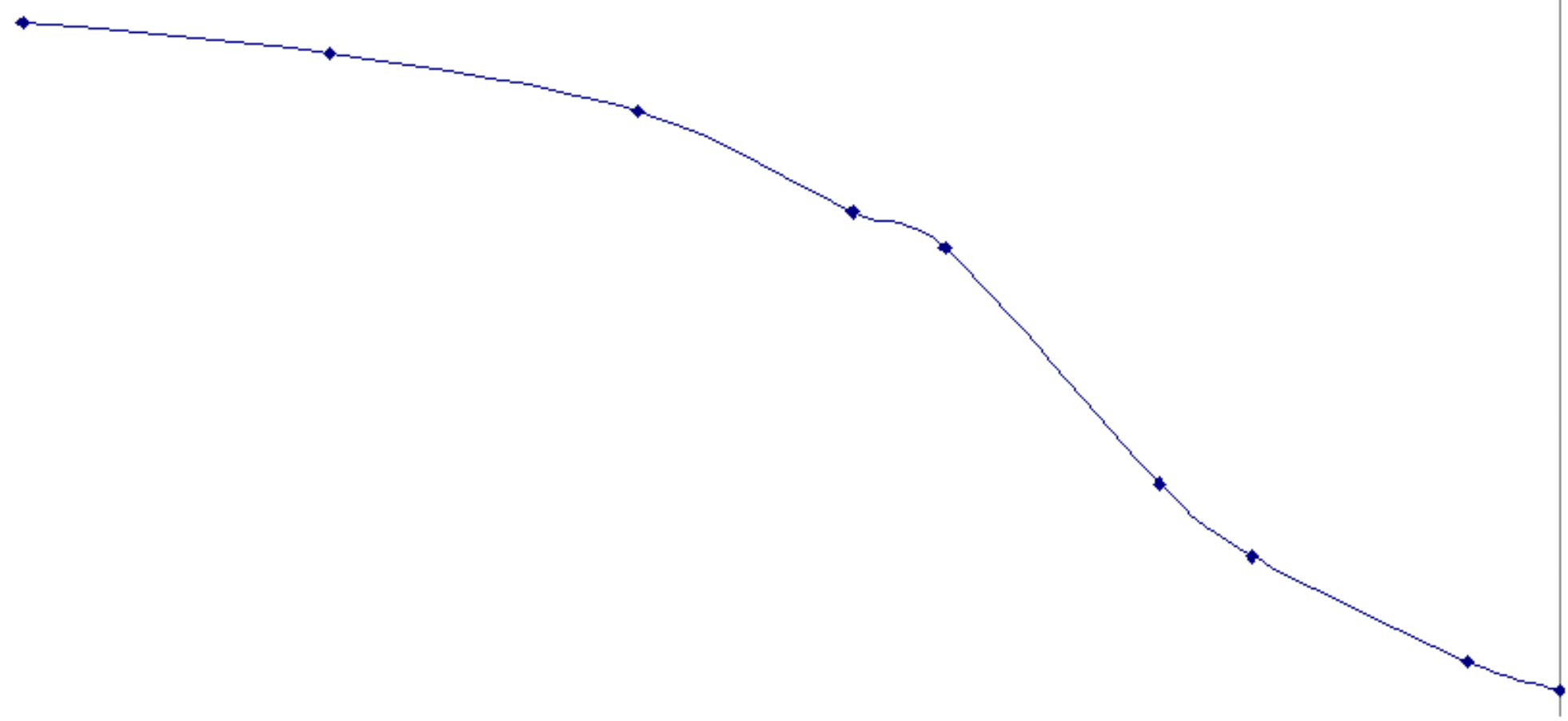




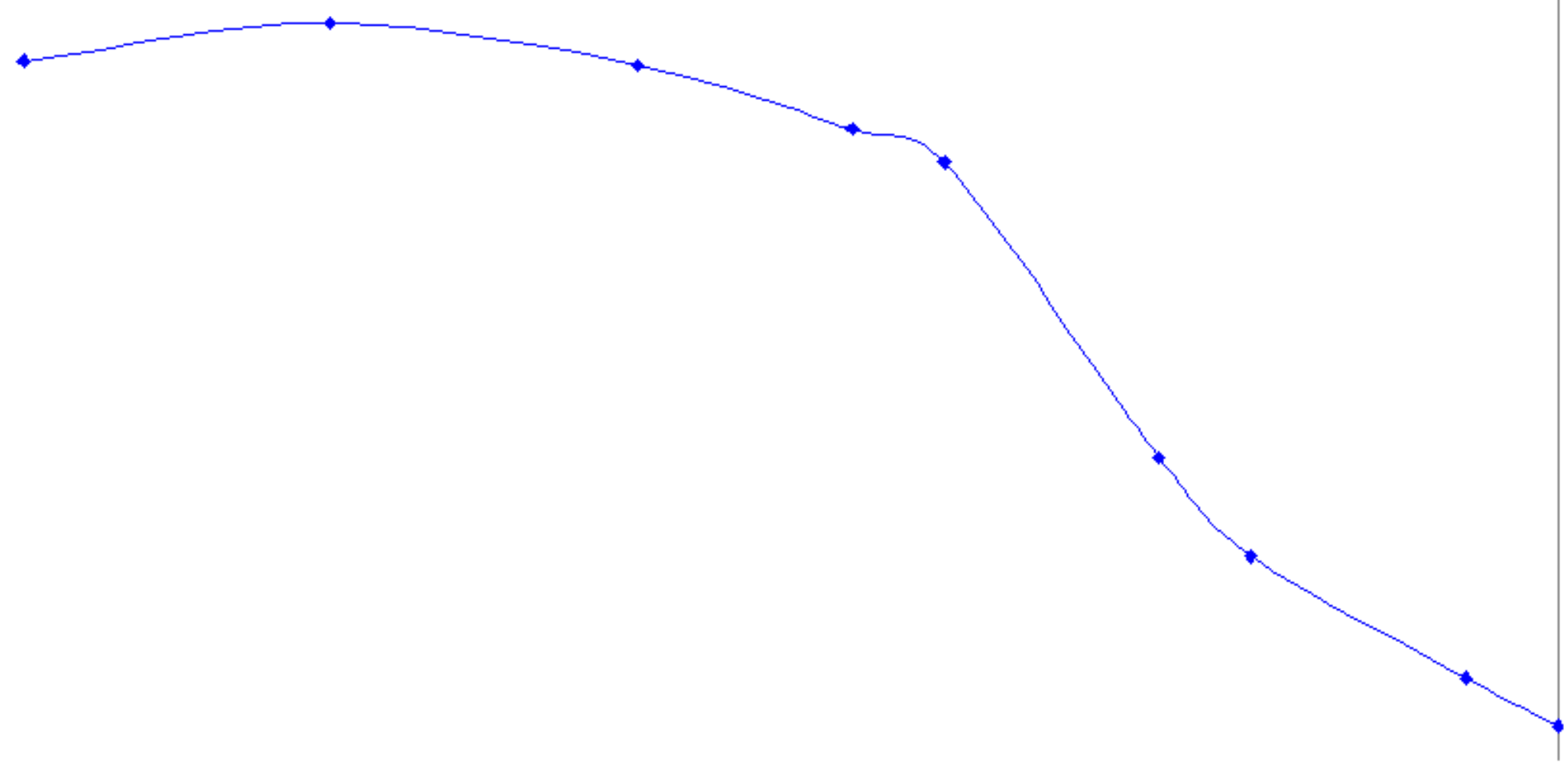




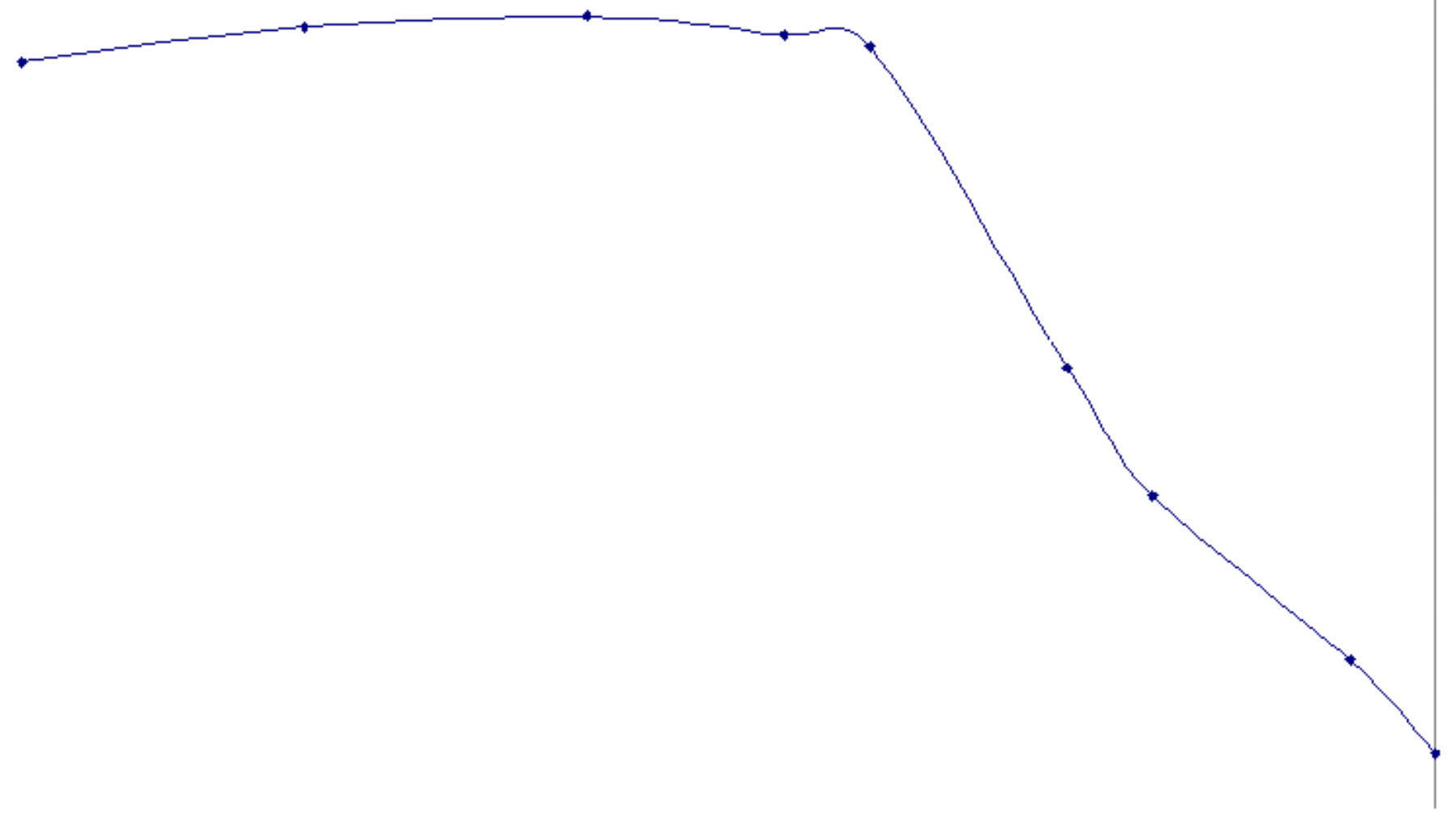




\section{Appendix M}

Calibration Curves for RAM 225 Mold Release on Aluminum Panels and Stainless Steel Disks

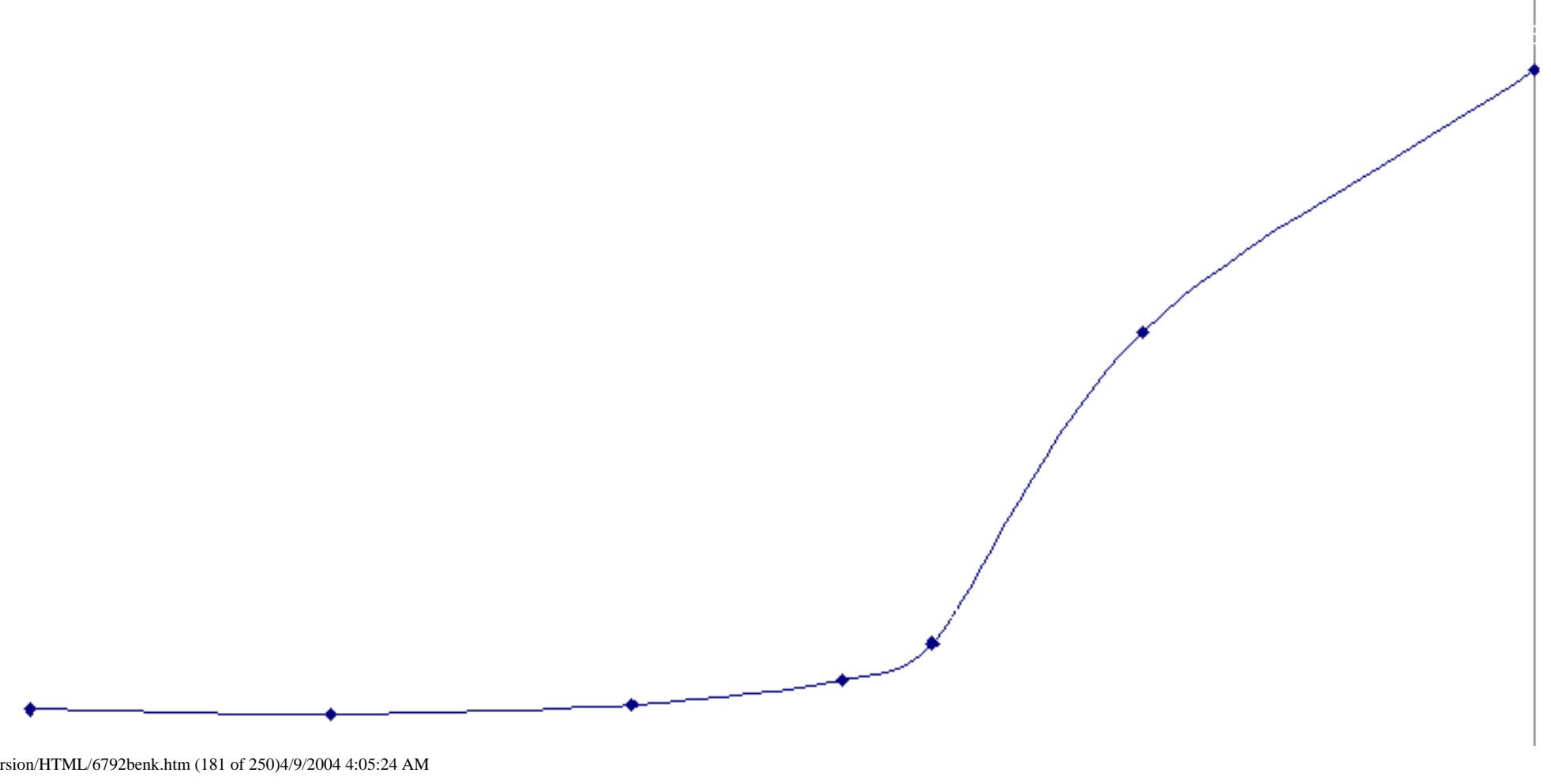




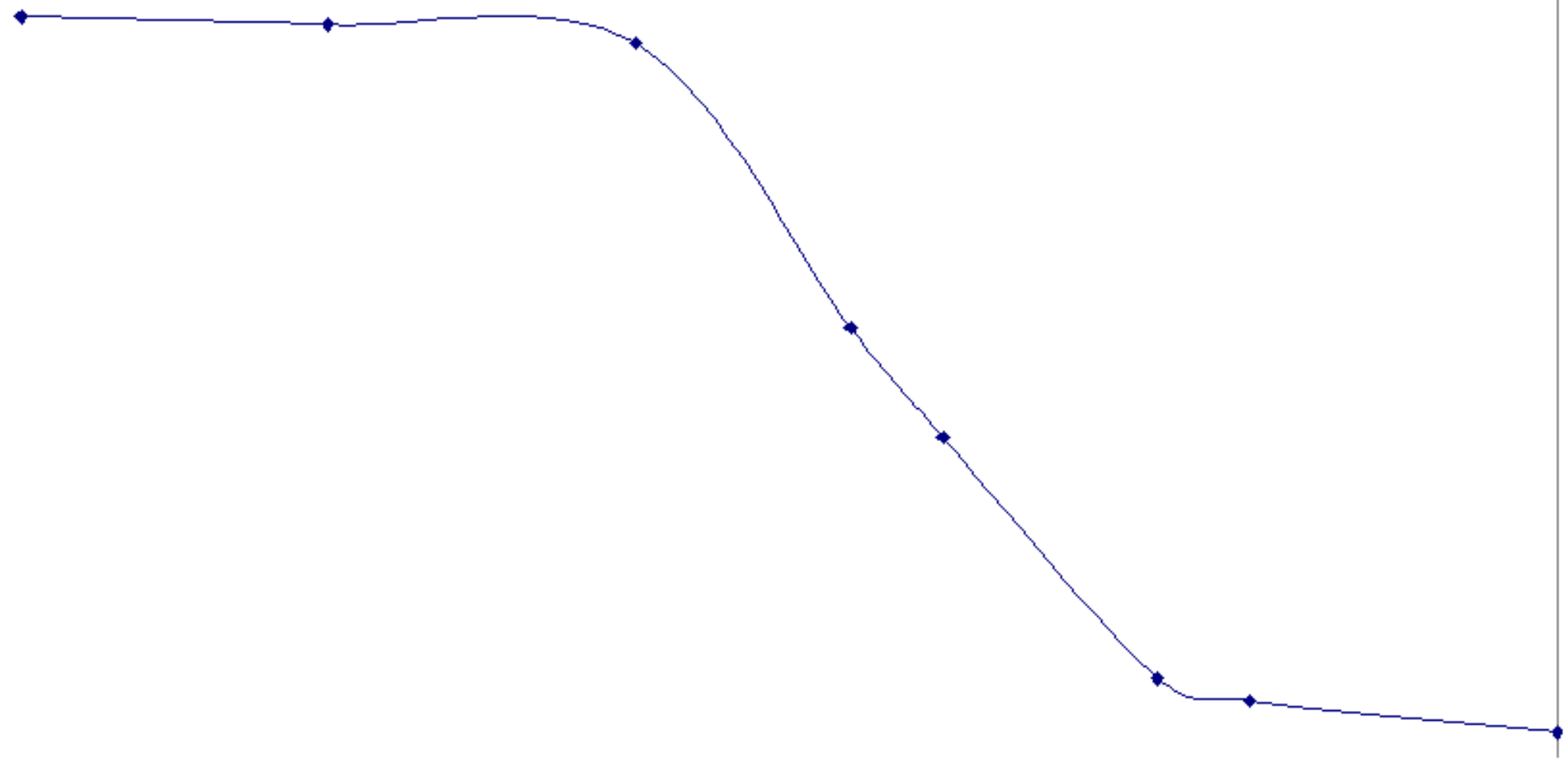

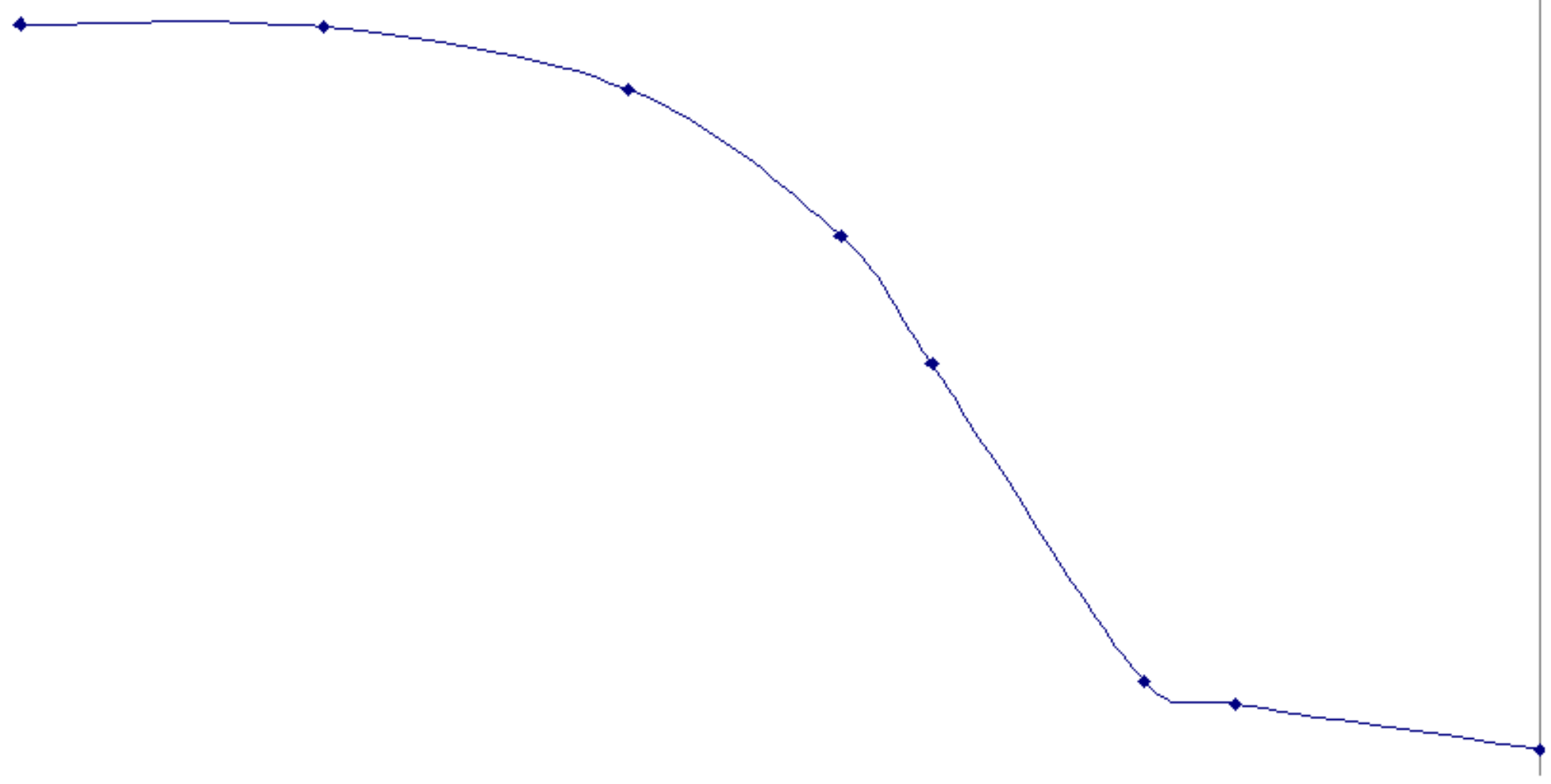
FY98 Annual Report

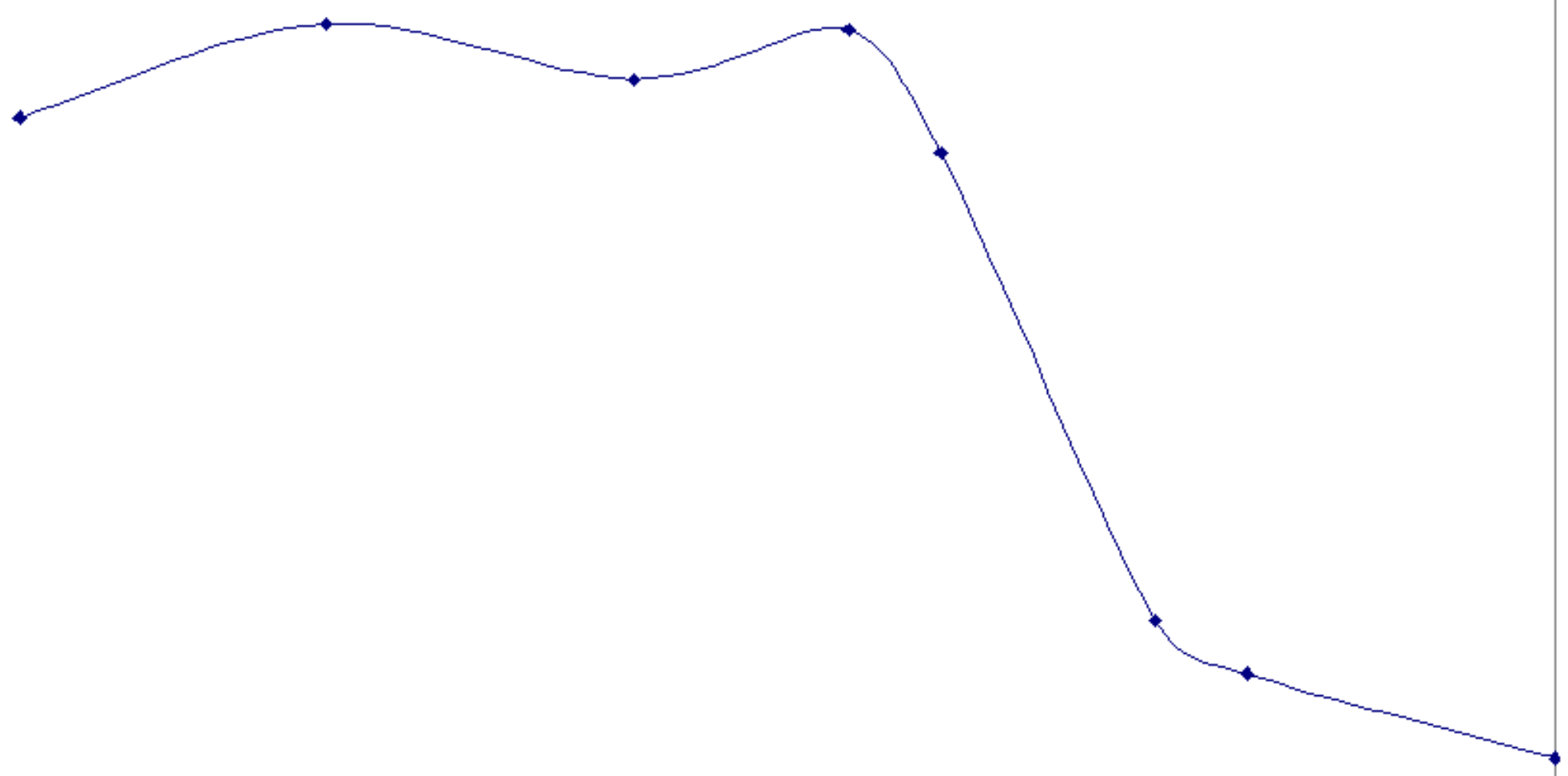

file:///C|/ManualConversion/HTML/6792benk.htm (185 of 250)4/9/2004 4:05:25 AM 
<smiles>C#CCCC</smiles> 

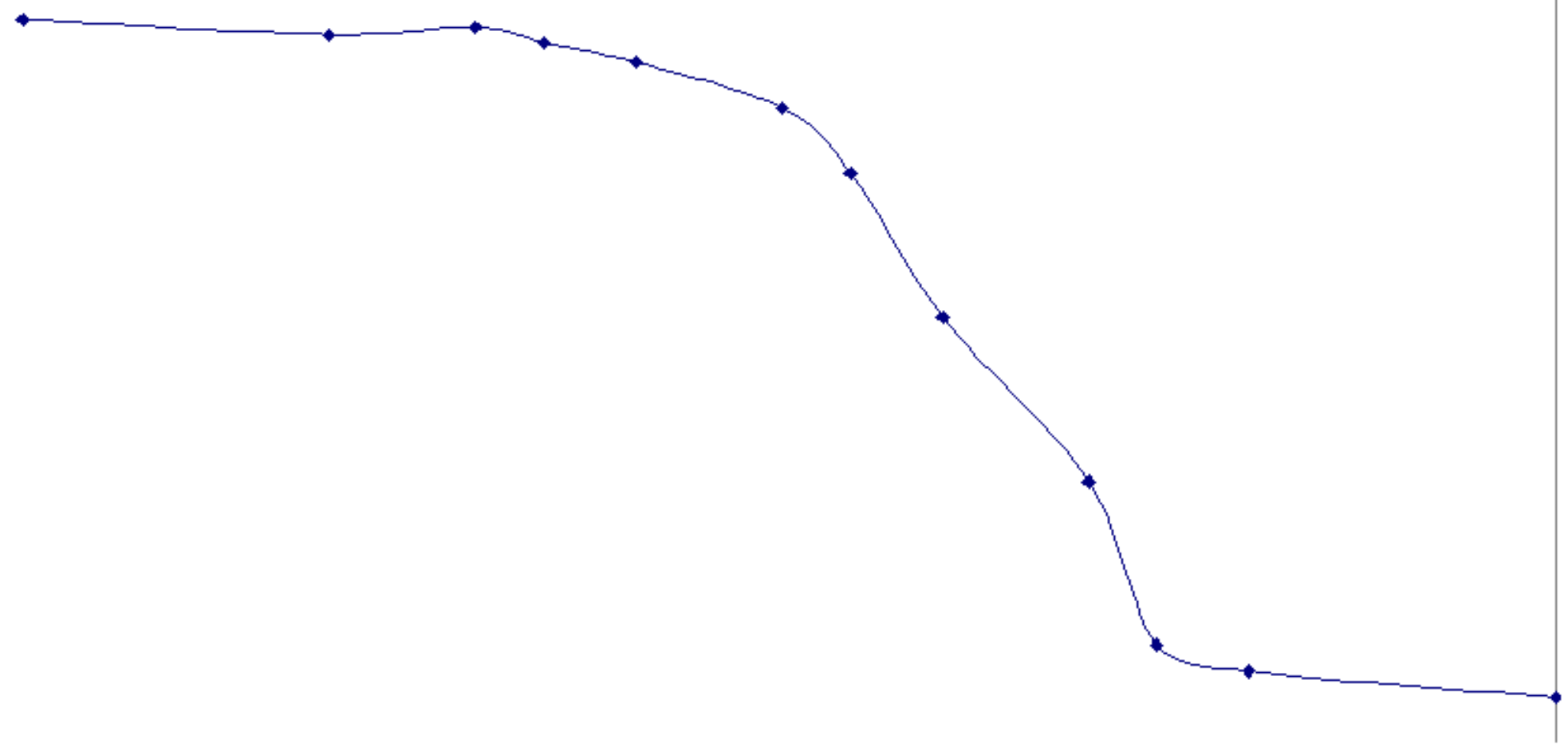

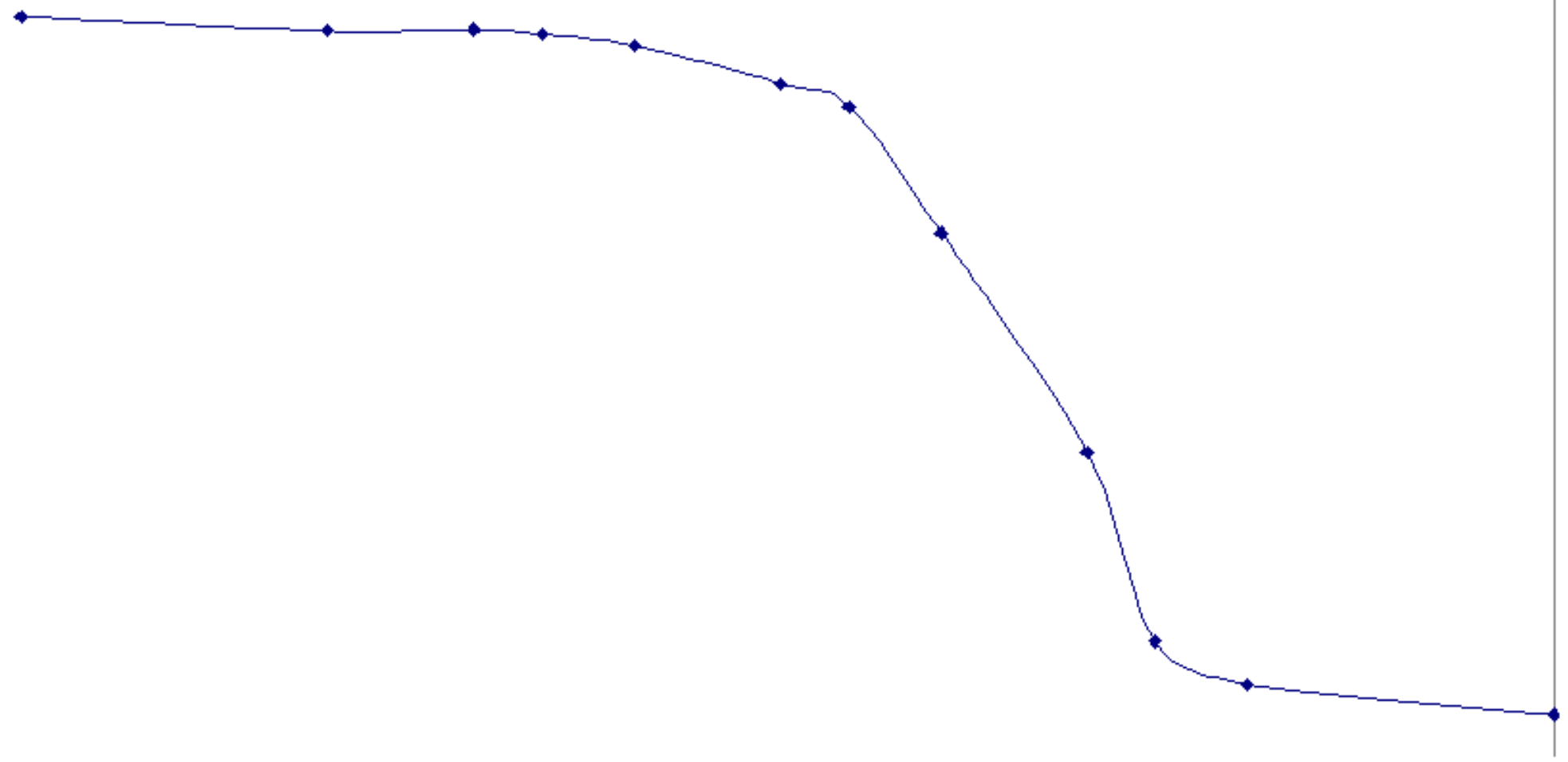


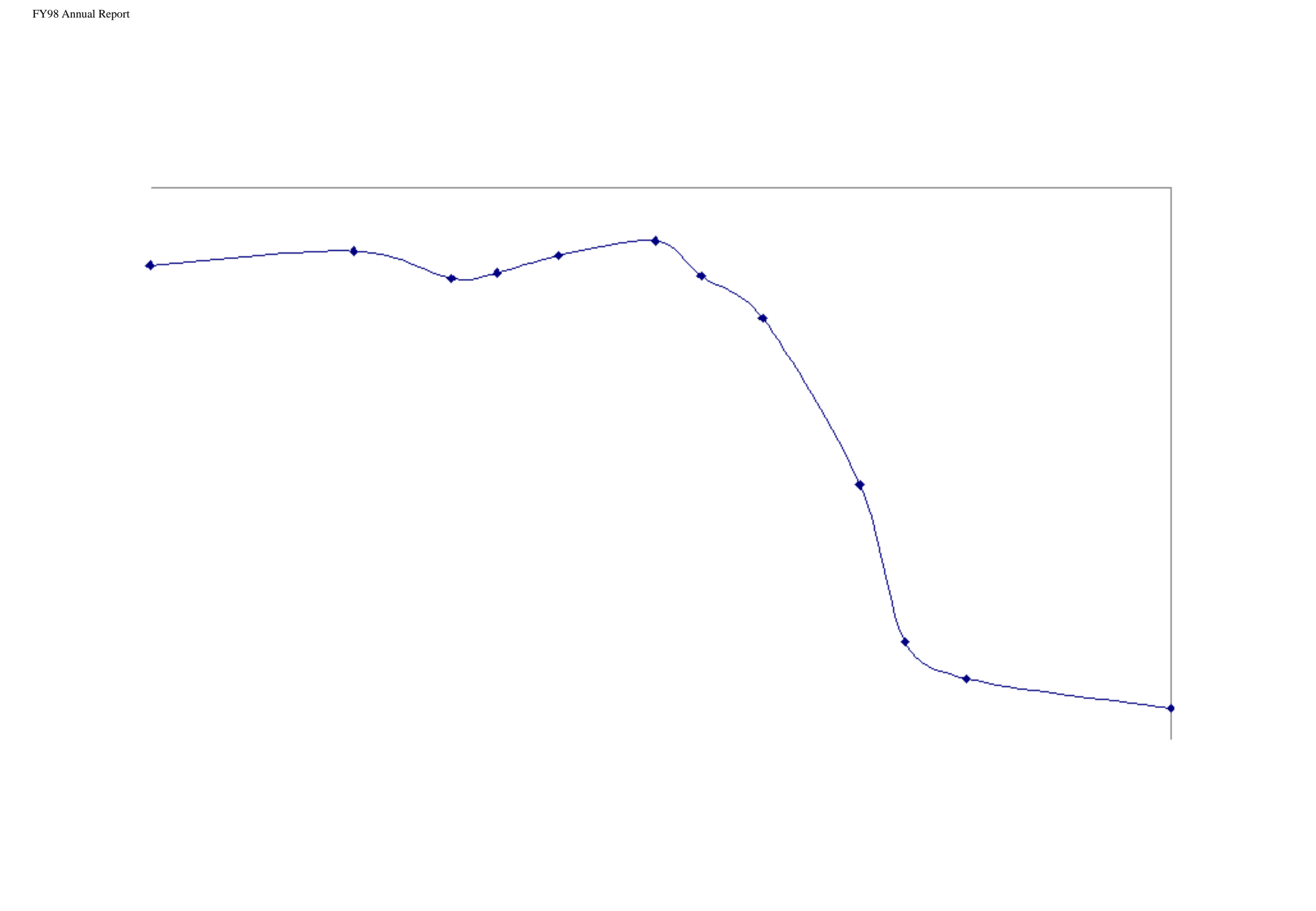


<smiles>CCCCCC</smiles> 


\section{Appendix N}

\section{Calibration Curves for Krylon Clear Covercoat Spray}

on Aluminum Panels and Stainless Steel Disks 
FY98 Annual Report

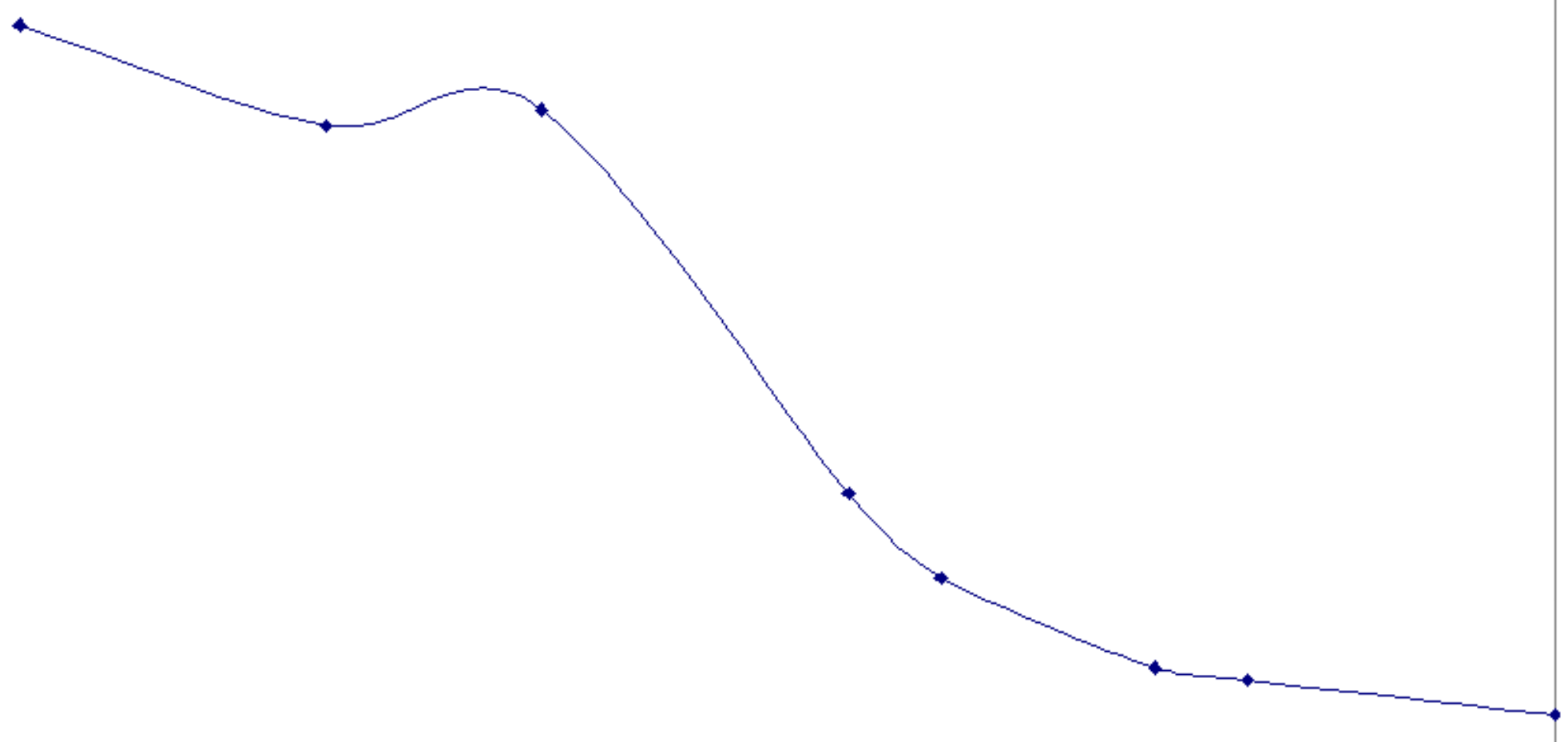

file://C|/ManualConversion/HTML/6792benk.htm (192 of 250)4/9/2004 4:05:25 AM 
FY98 Annual Report

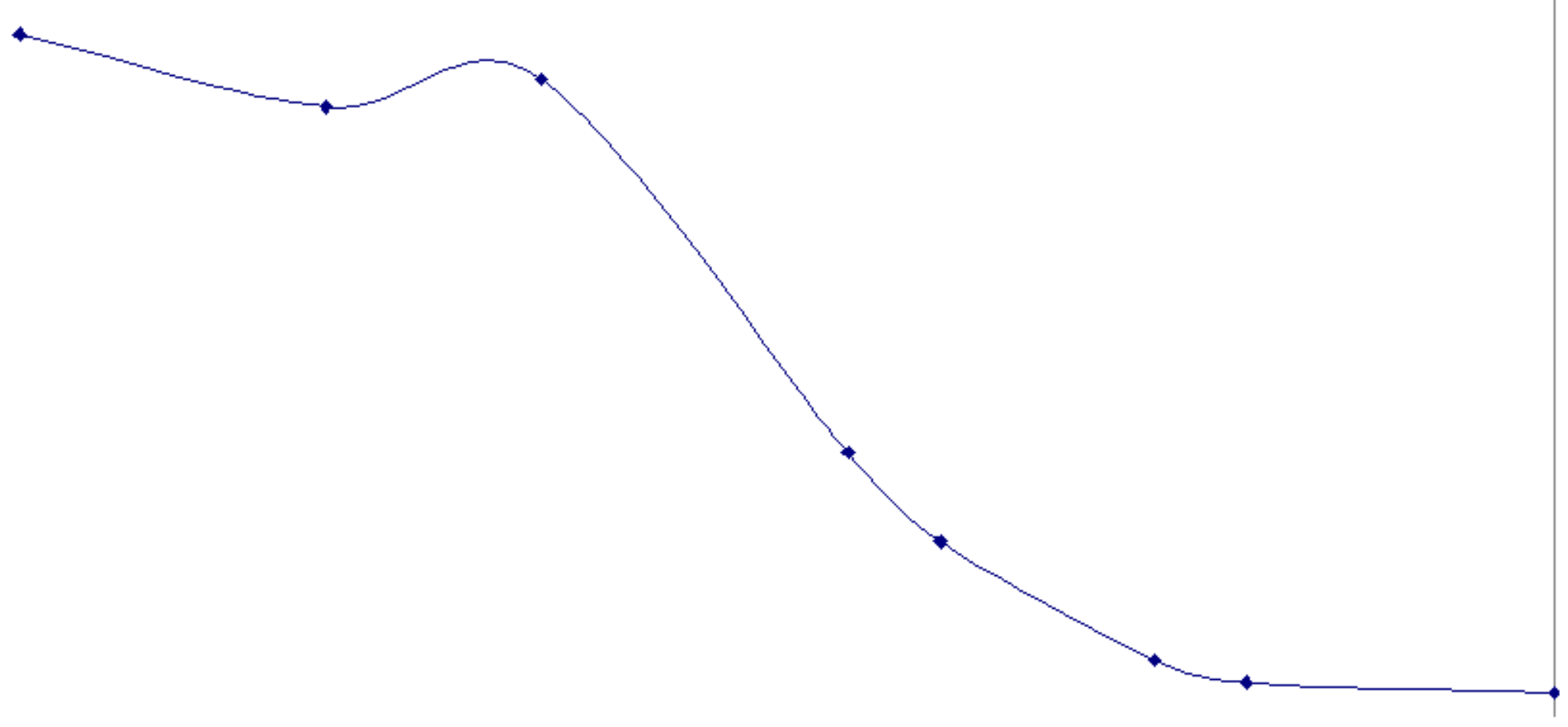

file://C//ManualConversion/HTML/6792benk.htm (193 of 250)4/9/2004 4:05:25 AM 

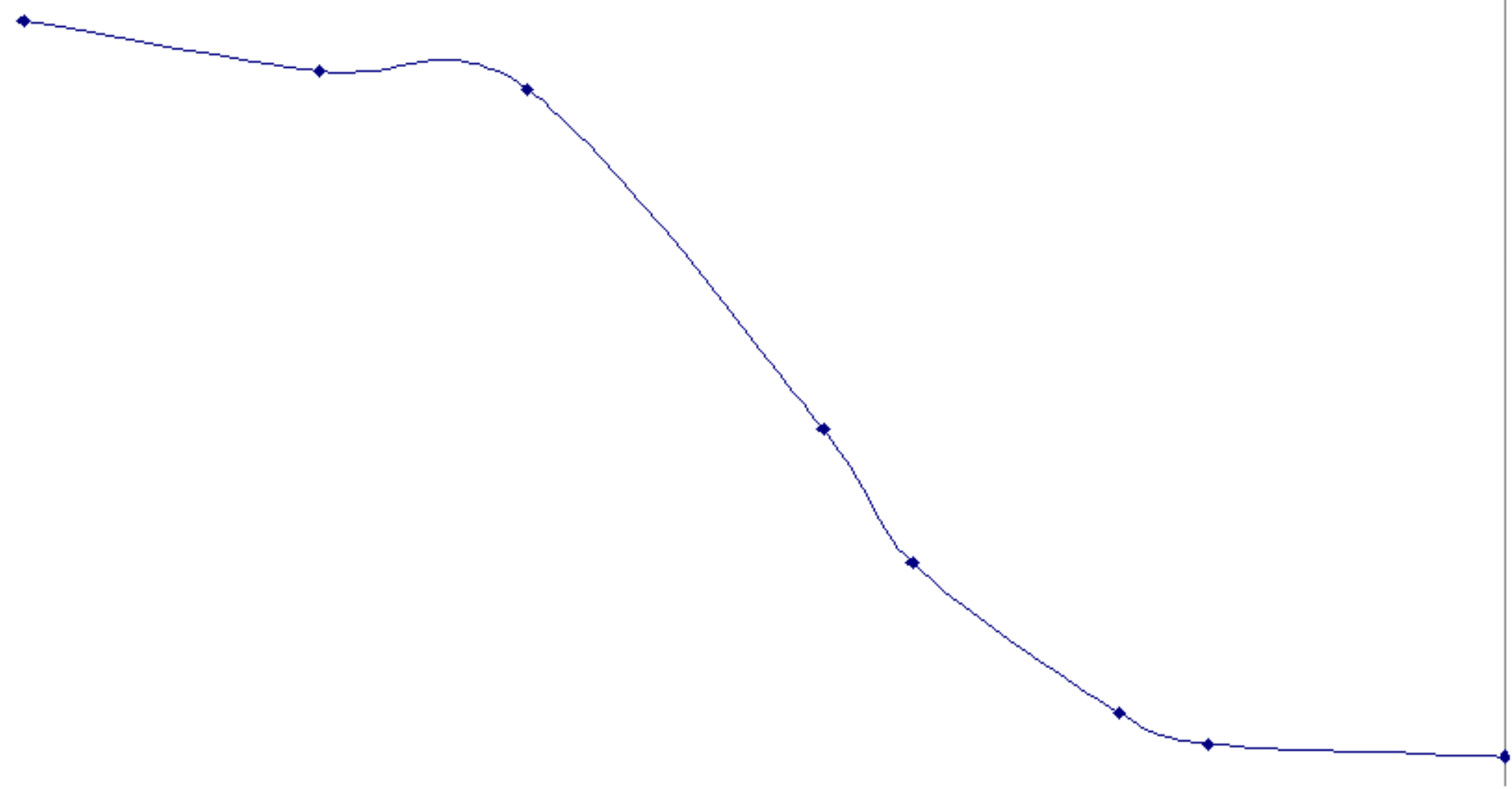


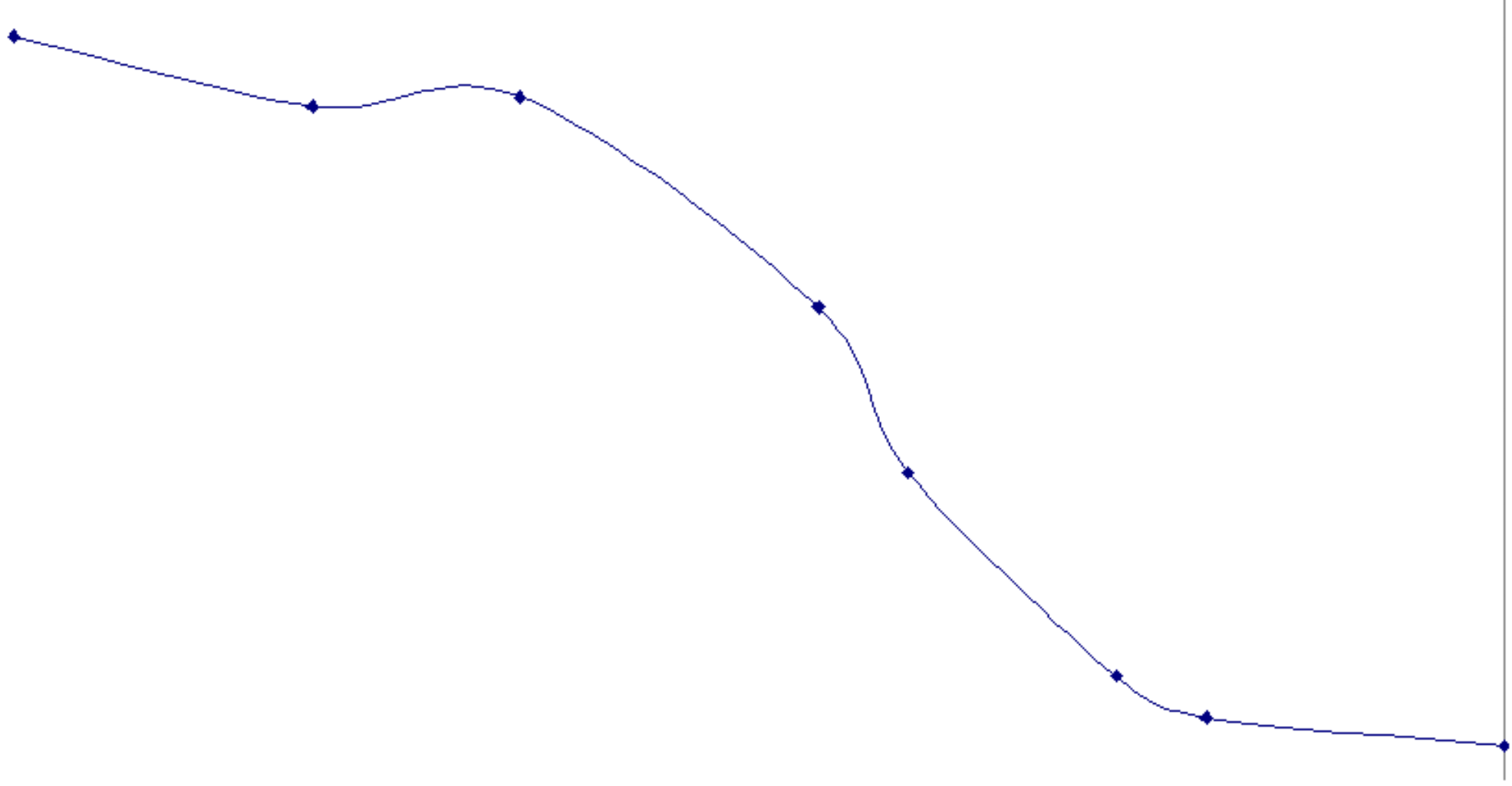




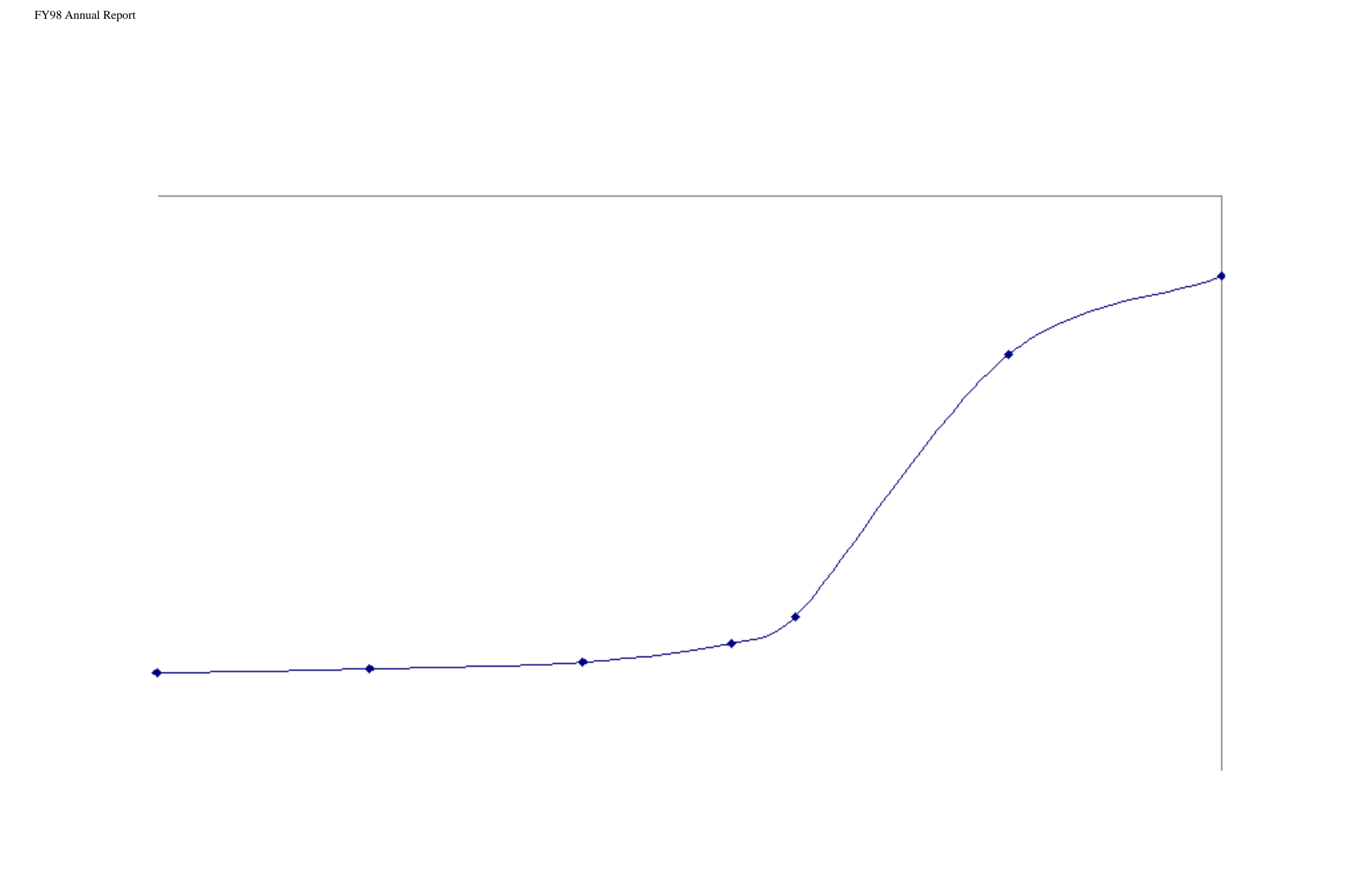


FY98 Annual Report

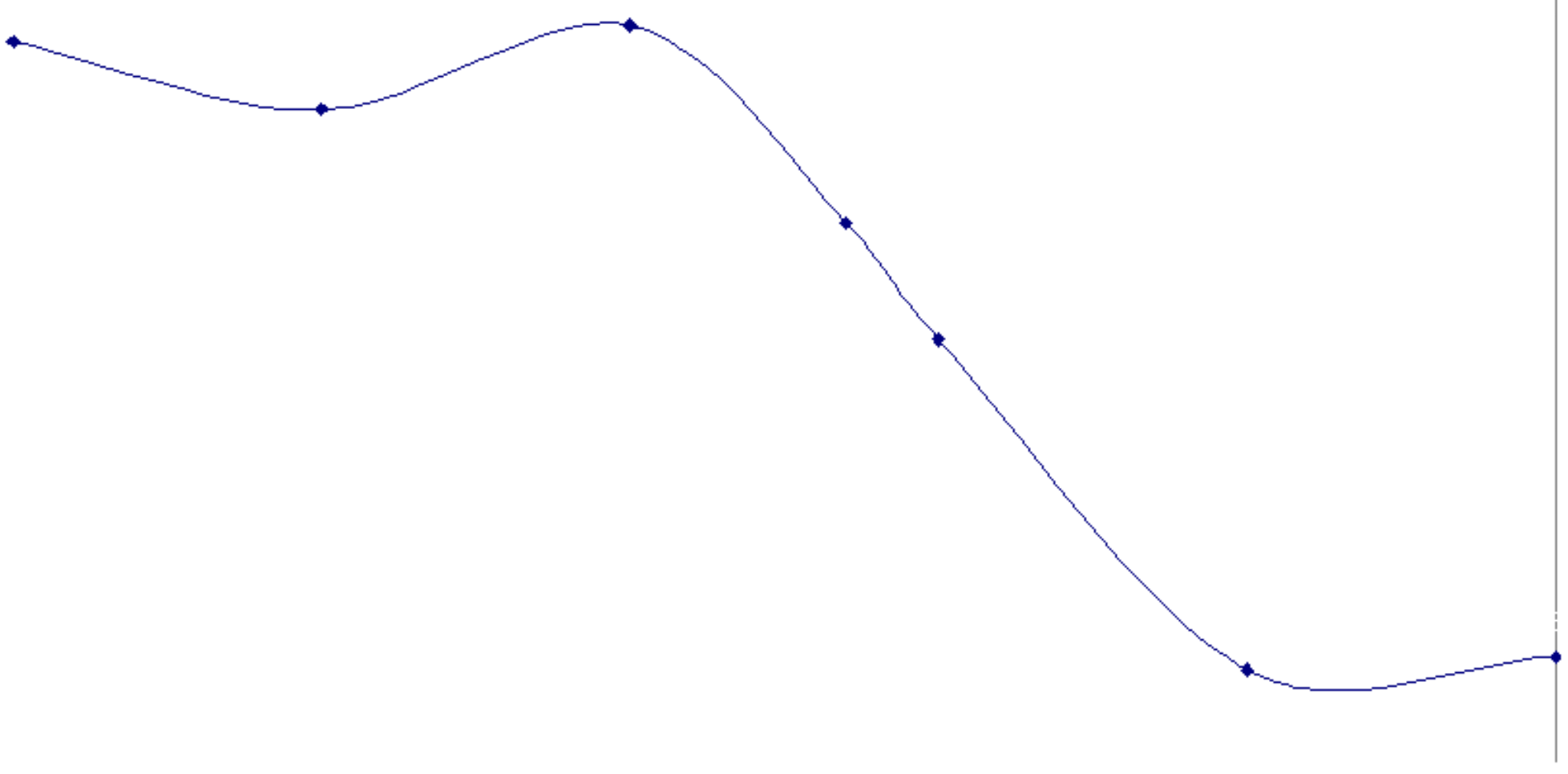

file:///C|/ManualConversion/HTML/6792benk.htm (197 of 250)4/9/2004 4:05:25 AM 
<smiles>CCCCCC</smiles> 


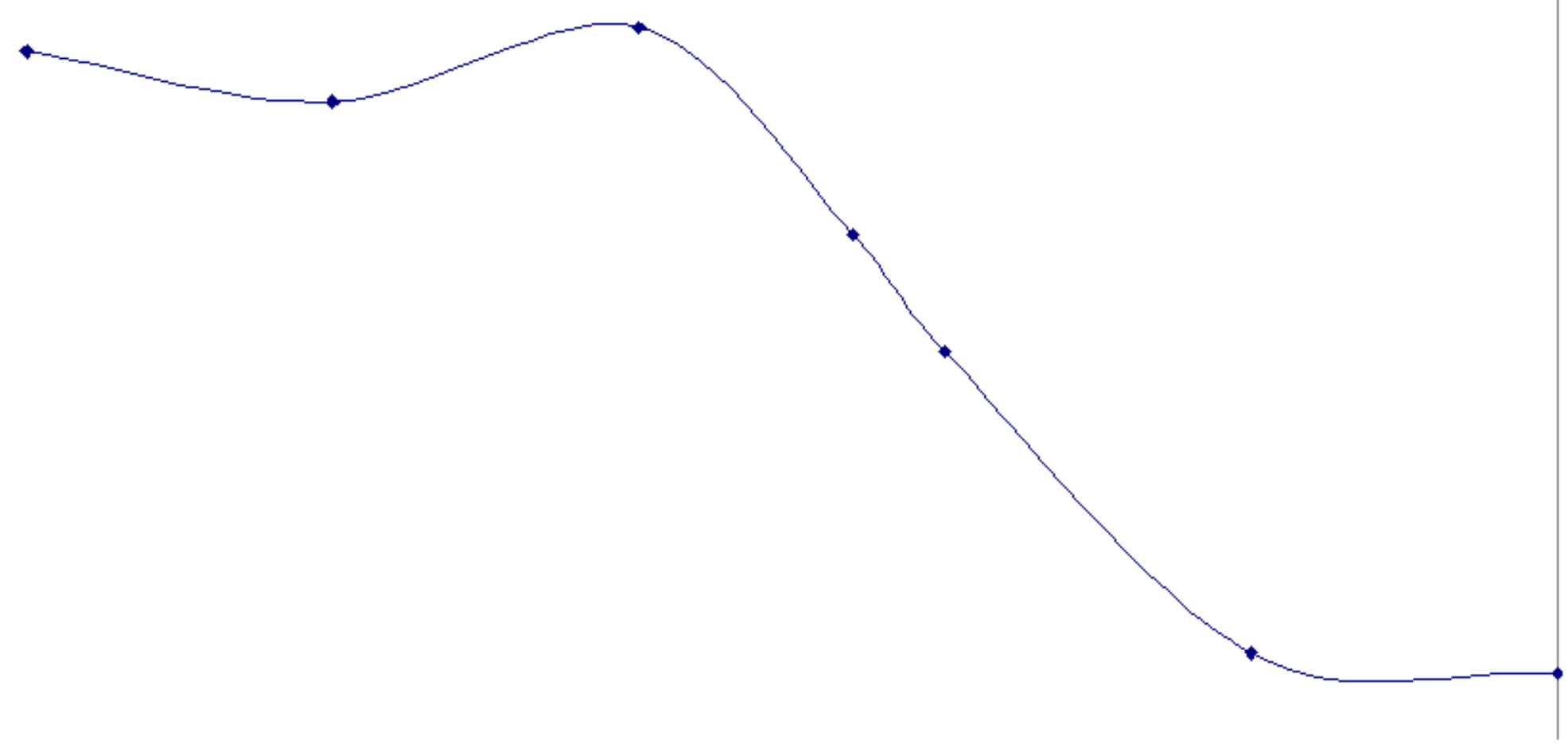




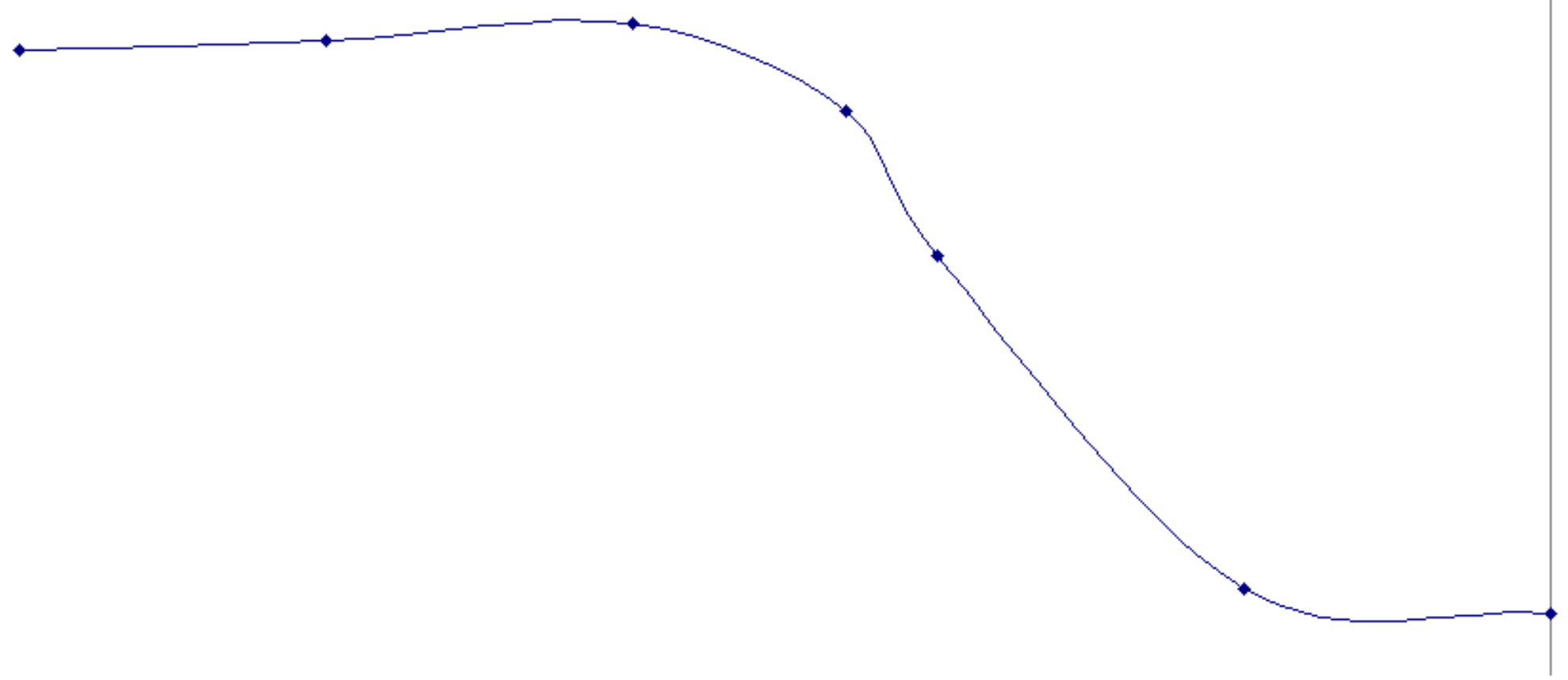




\section{Appendix O}

\section{Calibration Curves for EPON 828 Resin}

on Aluminum Panels and Stainless Steel Disks

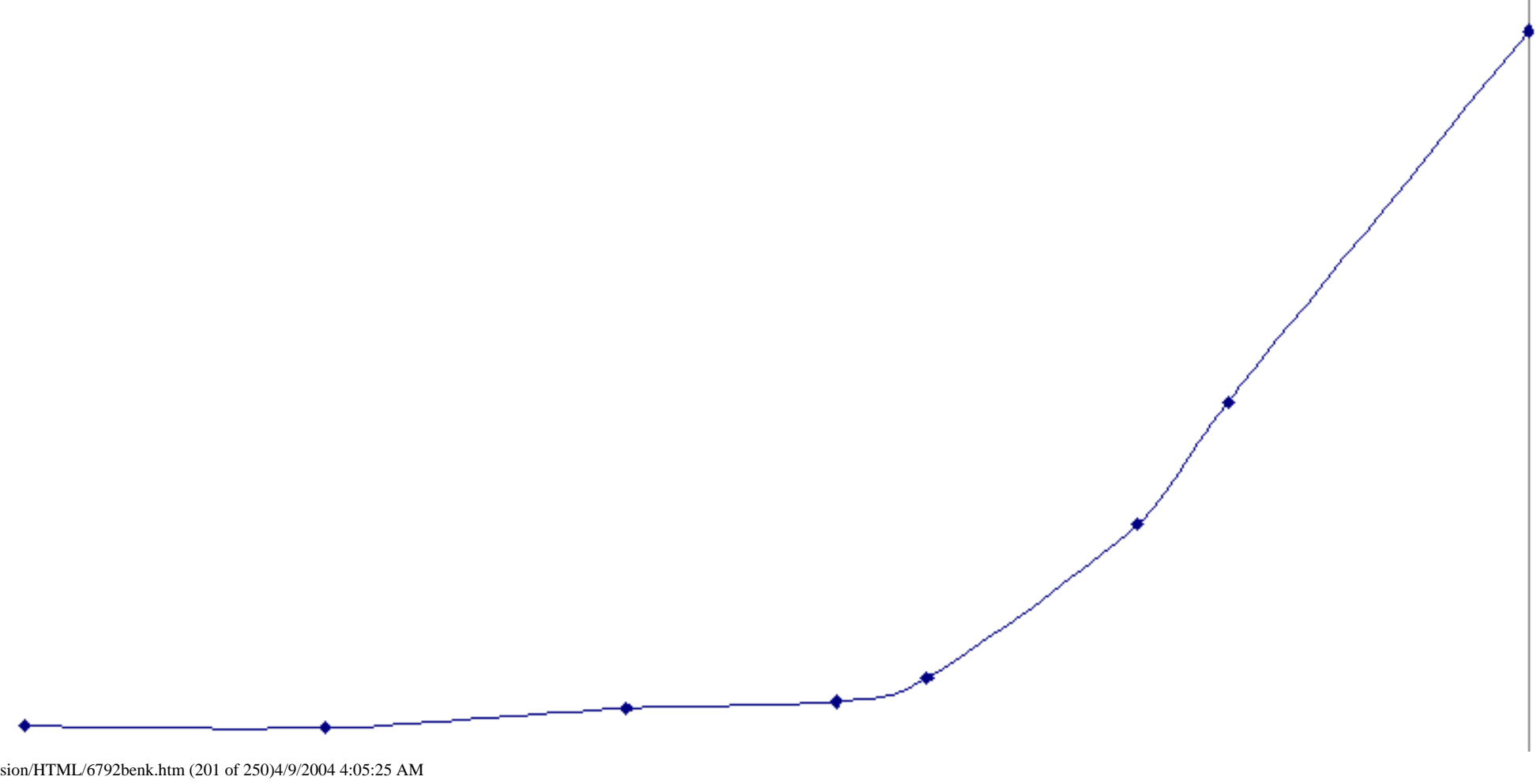


FY98 Annual Report

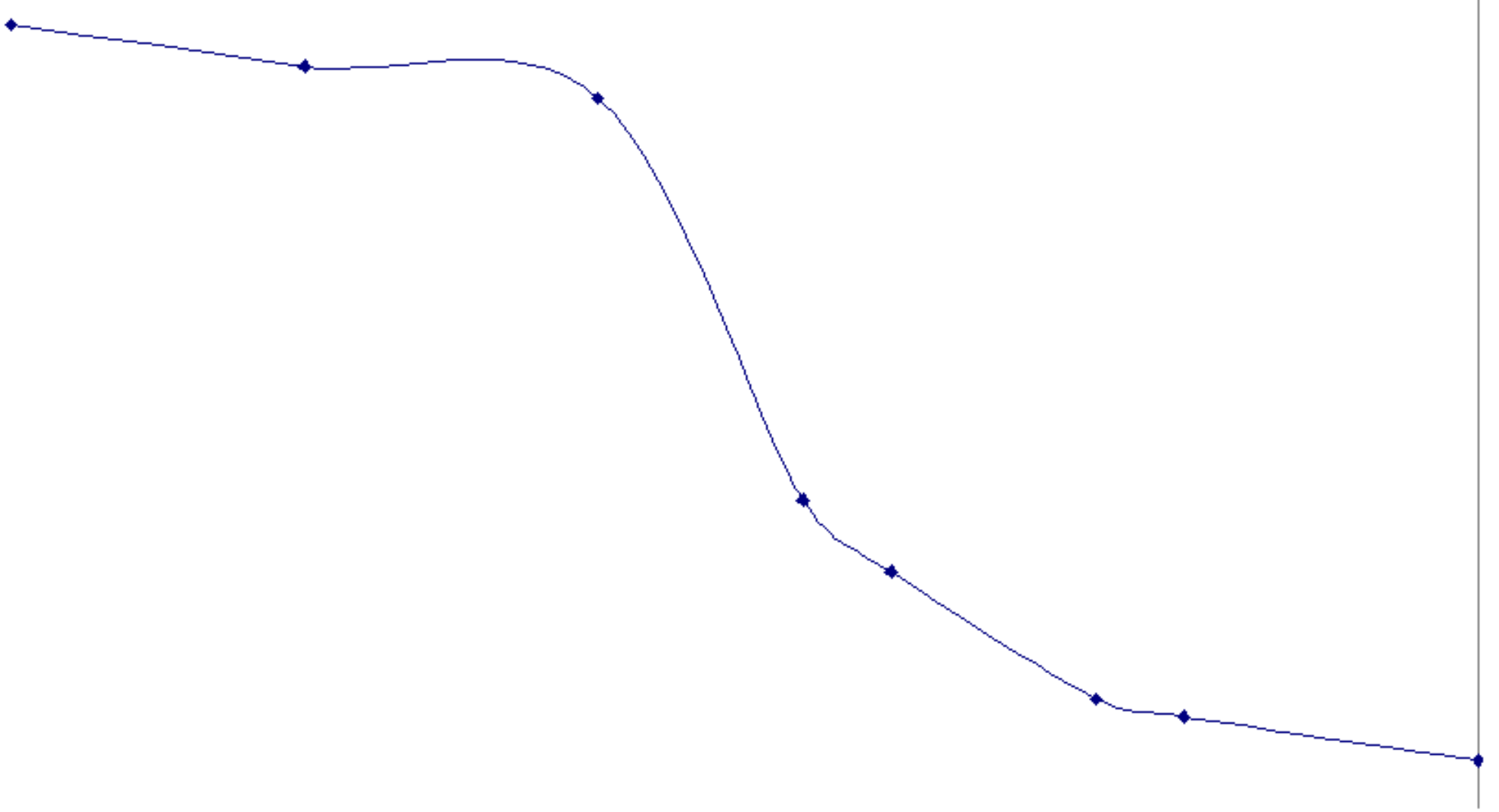

file://C//ManualConversion/HTML/6792benk.htm (202 of 250)4/9/2004 4:05:25 AM 
FY98 Annual Report

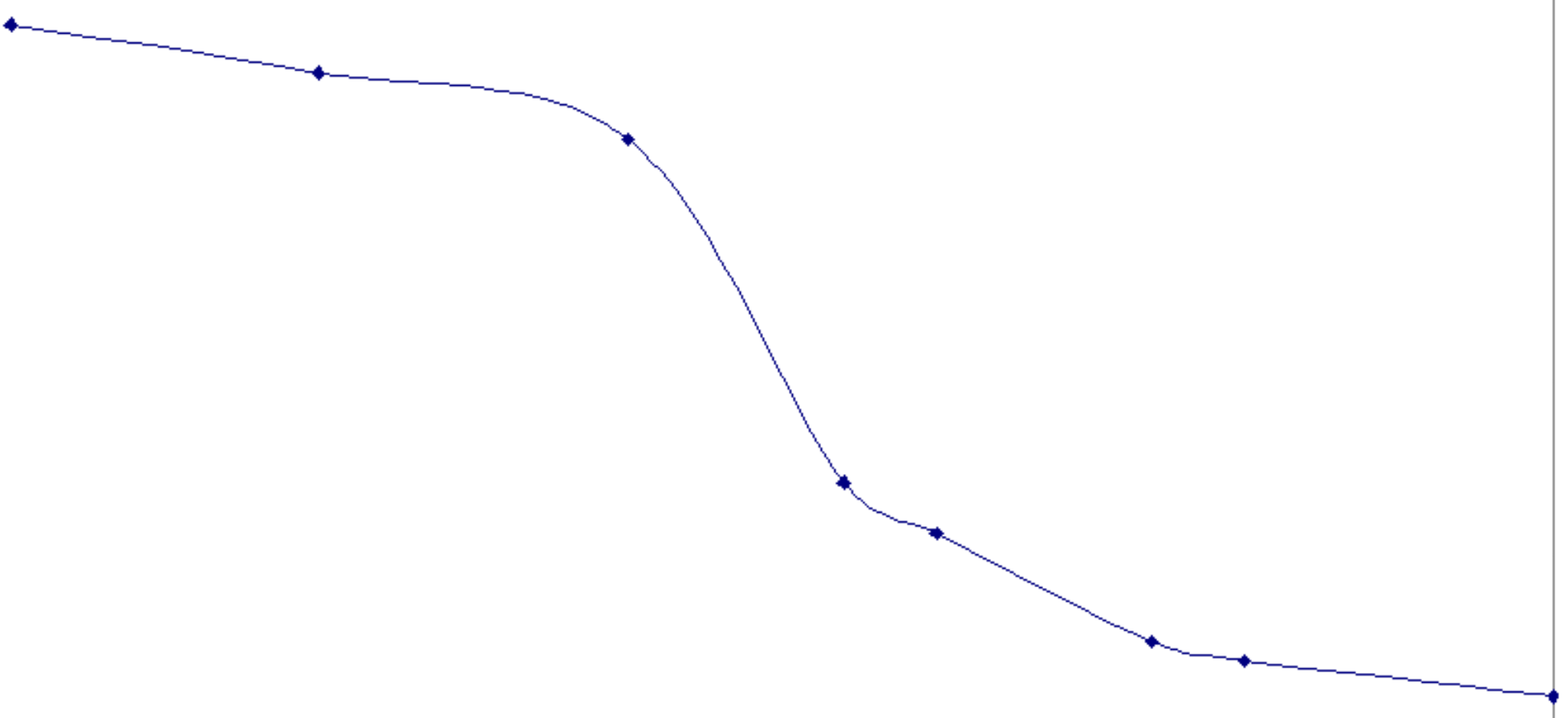

file://C//ManualConversion/HTML/6792benk.htm (203 of 250)4/9/2004 4:05:25 AM 
FY98 Annual Report

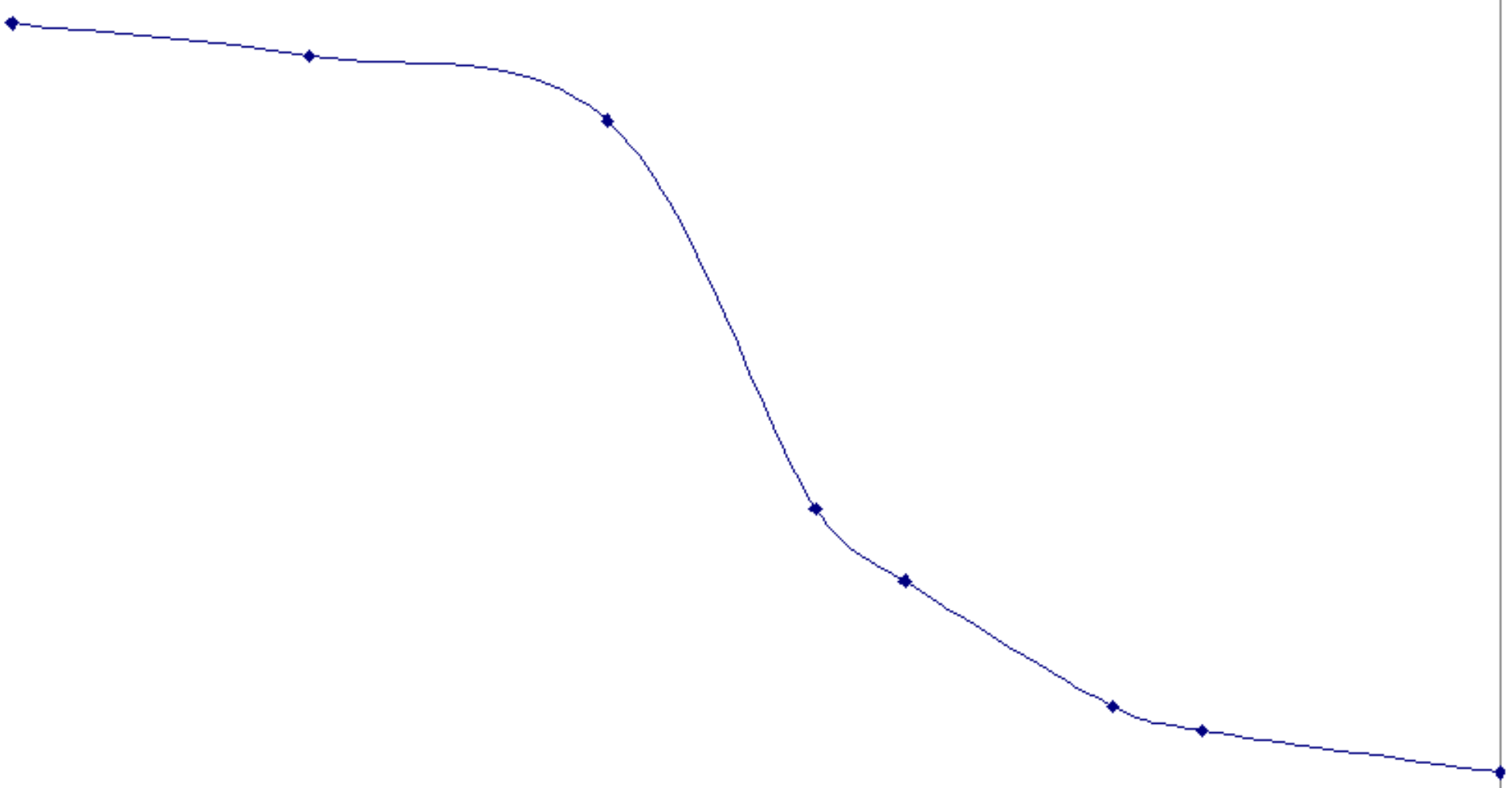

file://C//ManualConversion/HTML/6792benk.htm (204 of 250)4/9/2004 4:05:25 AM 
<smiles>CCCCC</smiles> 
<smiles>CCCC</smiles> 


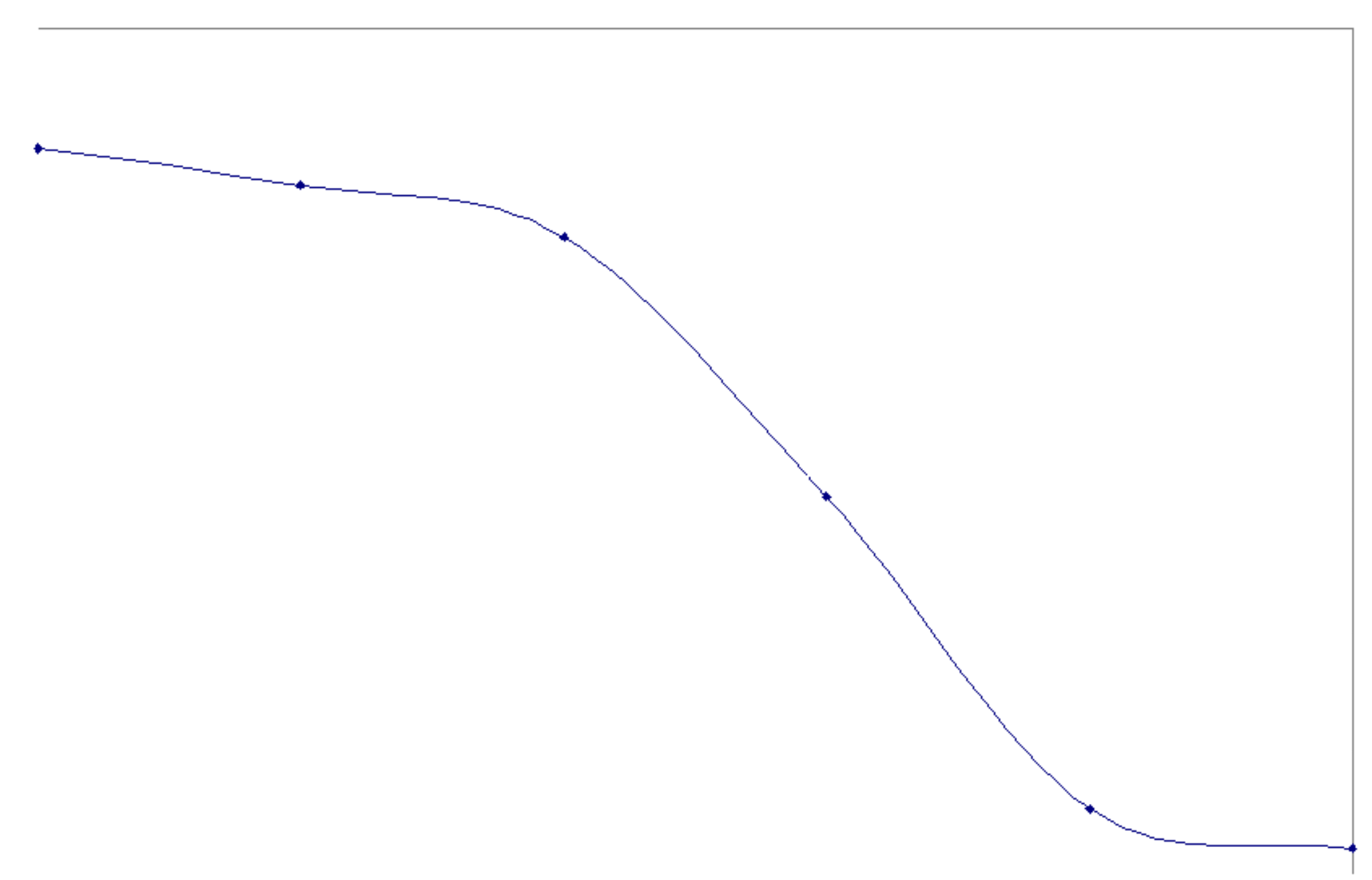


FY98 Annual Report

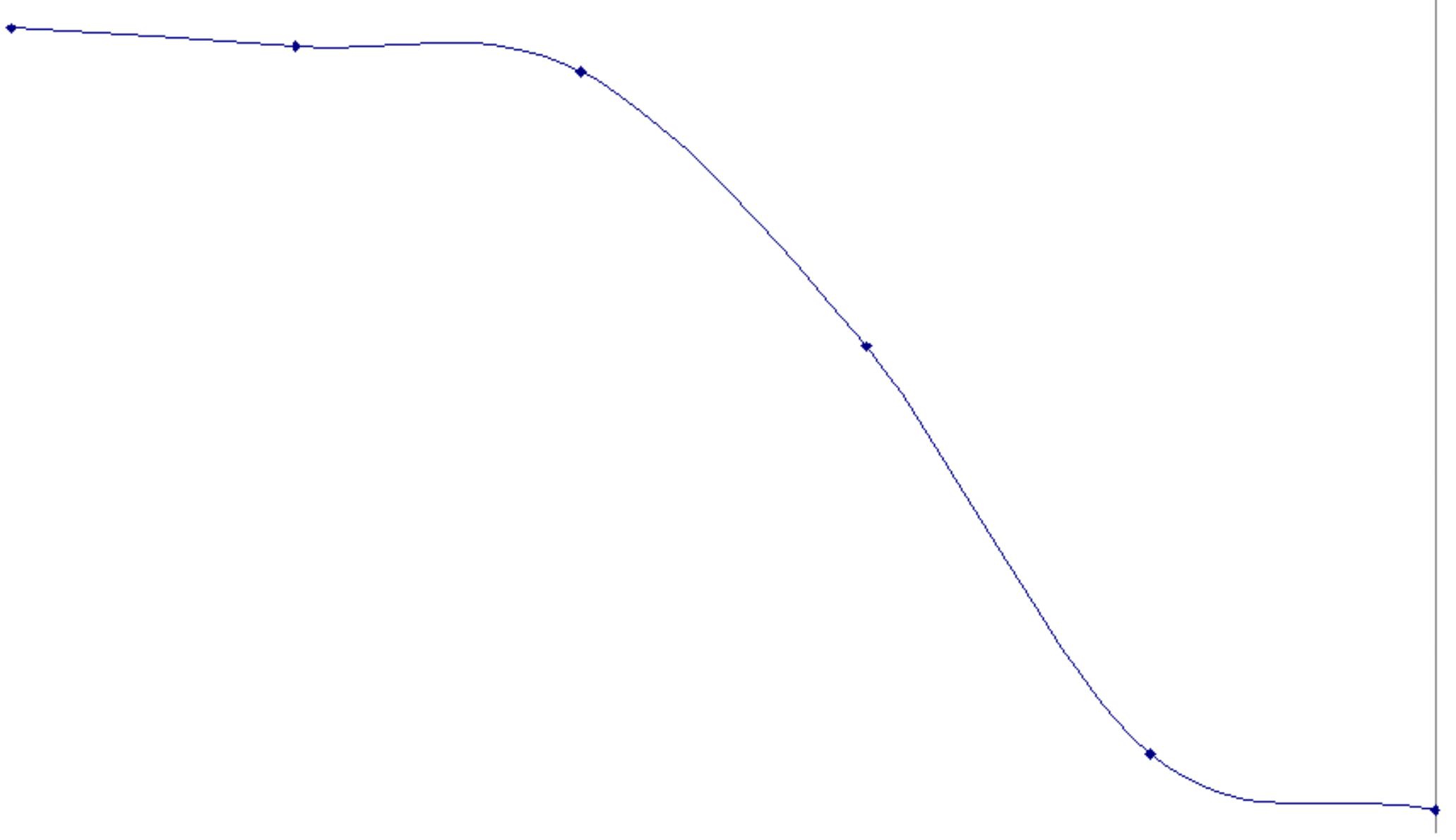

file:///C//ManualConversion/HTML/6792benk.htm (208 of 250)4/9/2004 4:05:25 AM 


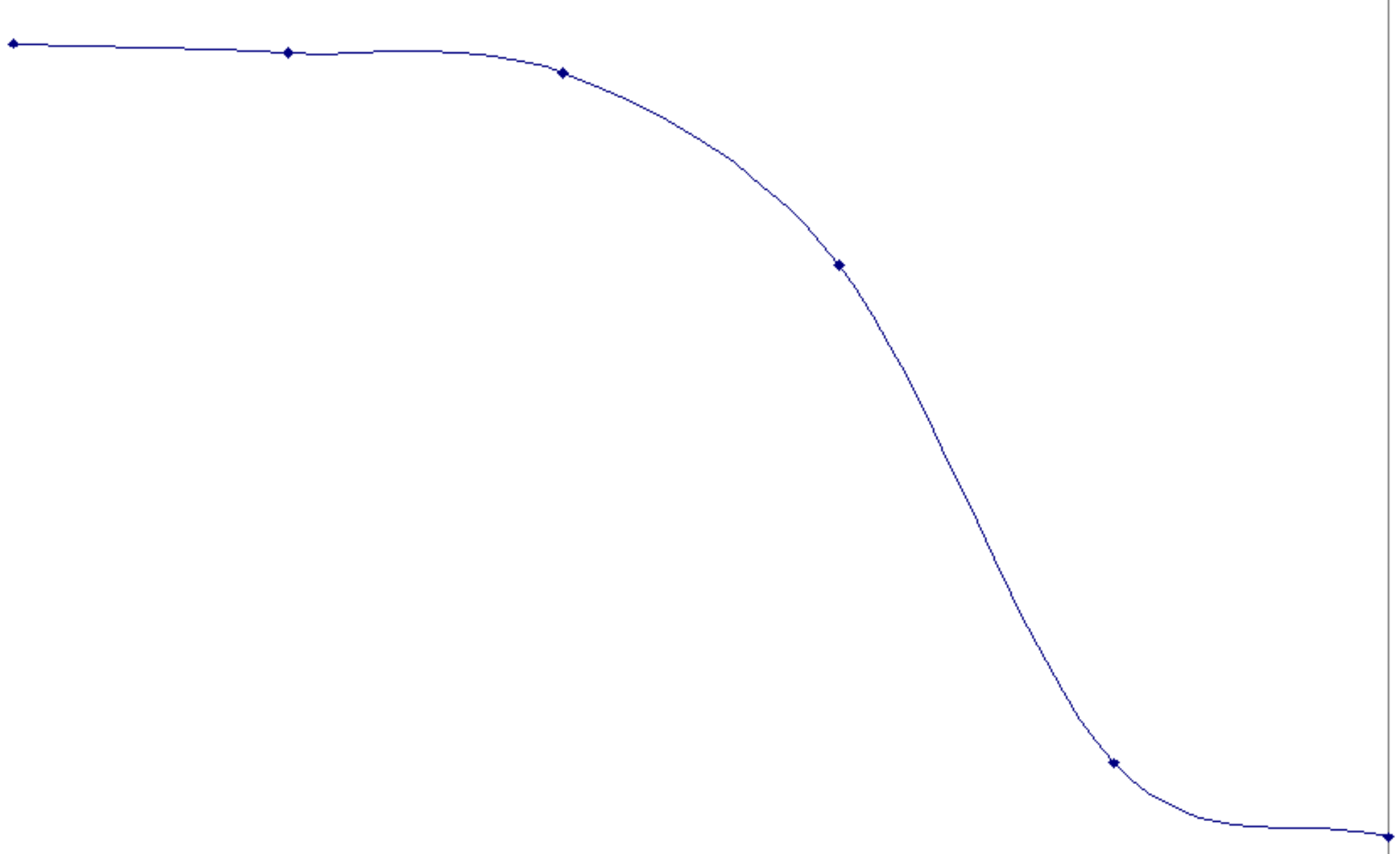



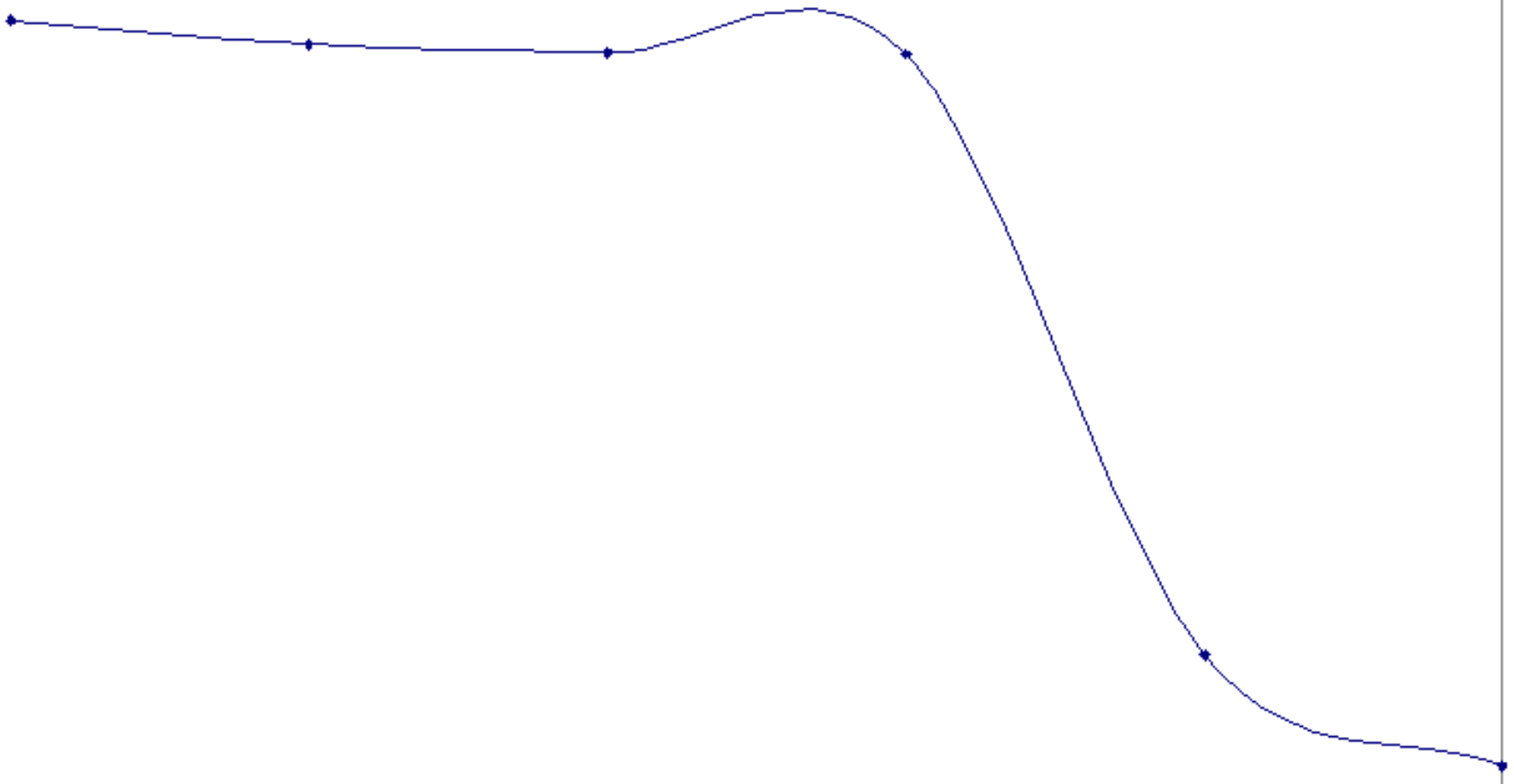


\section{Appendix P}

\section{Calibration Curves for Versamid 140 Curing Agent} on Aluminum Panels and Stainless Steel Disks 

$+$

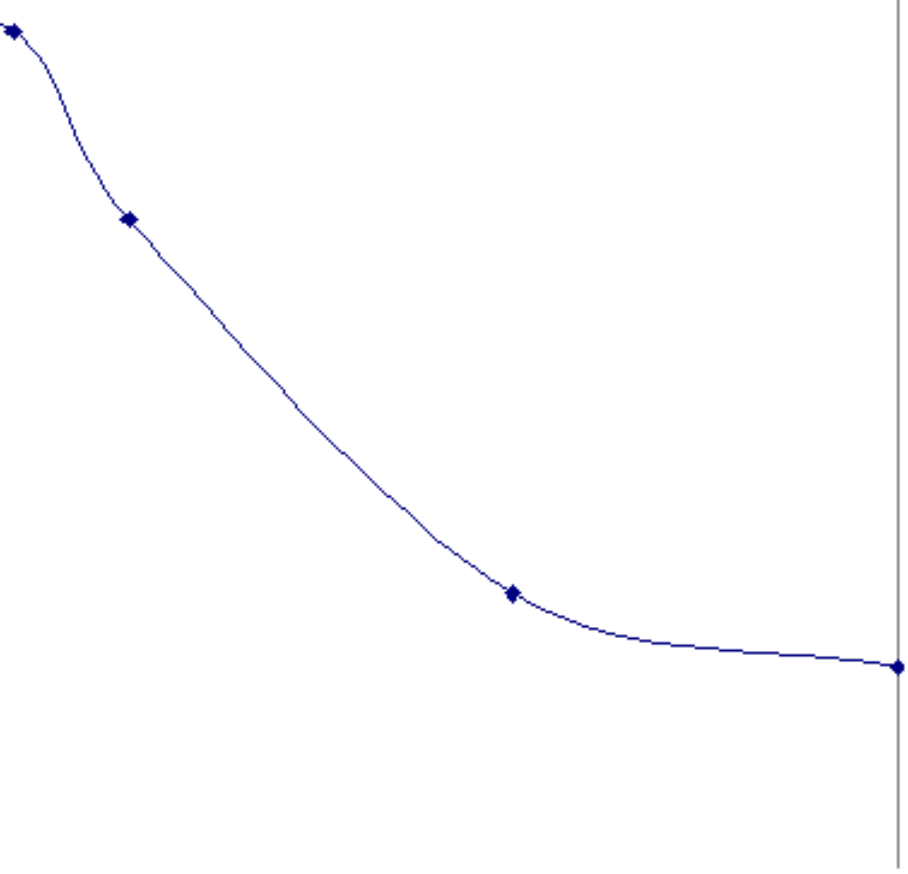


<smiles>CCCCCC</smiles> 


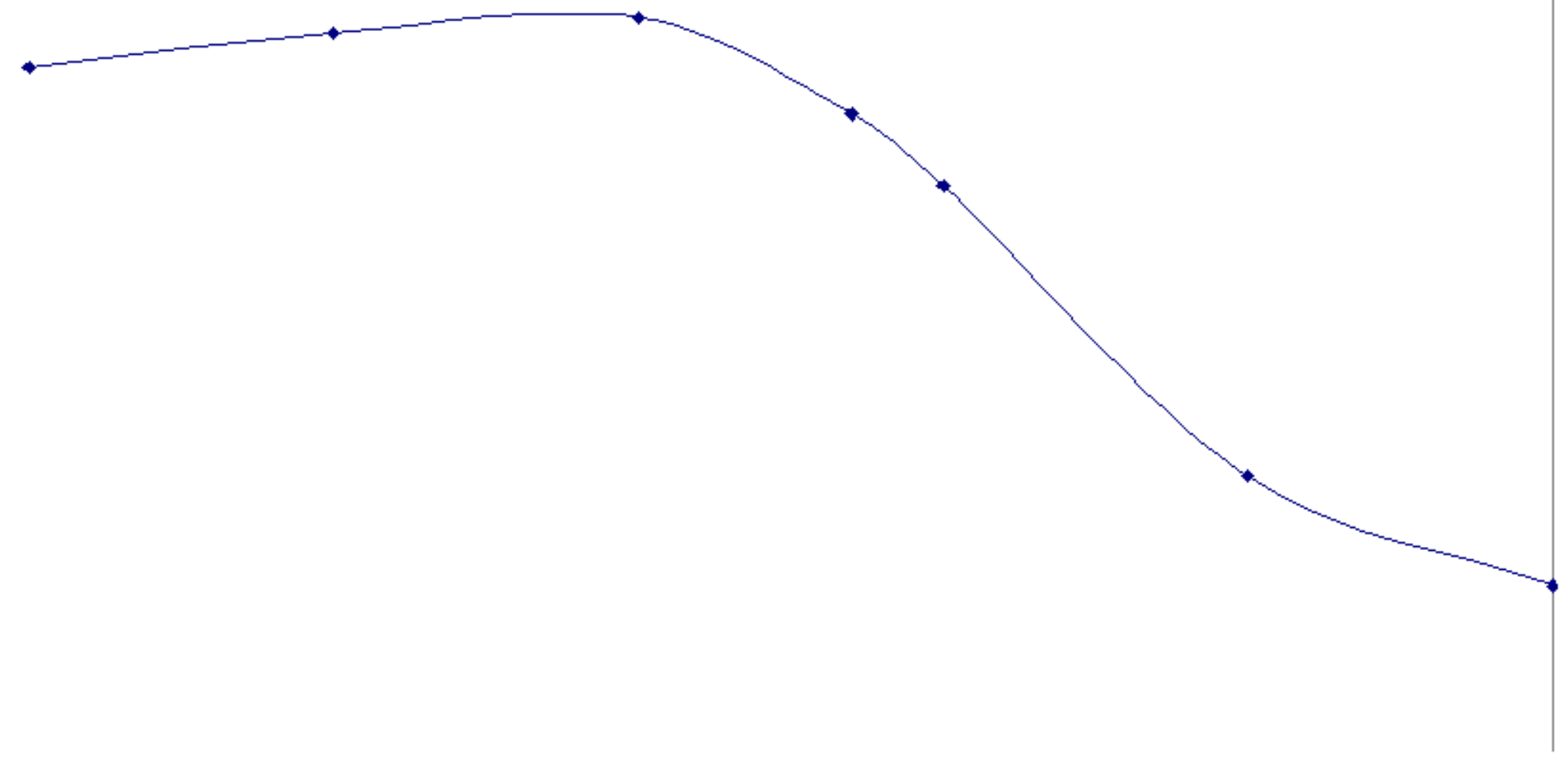


<smiles>CCCC</smiles> 


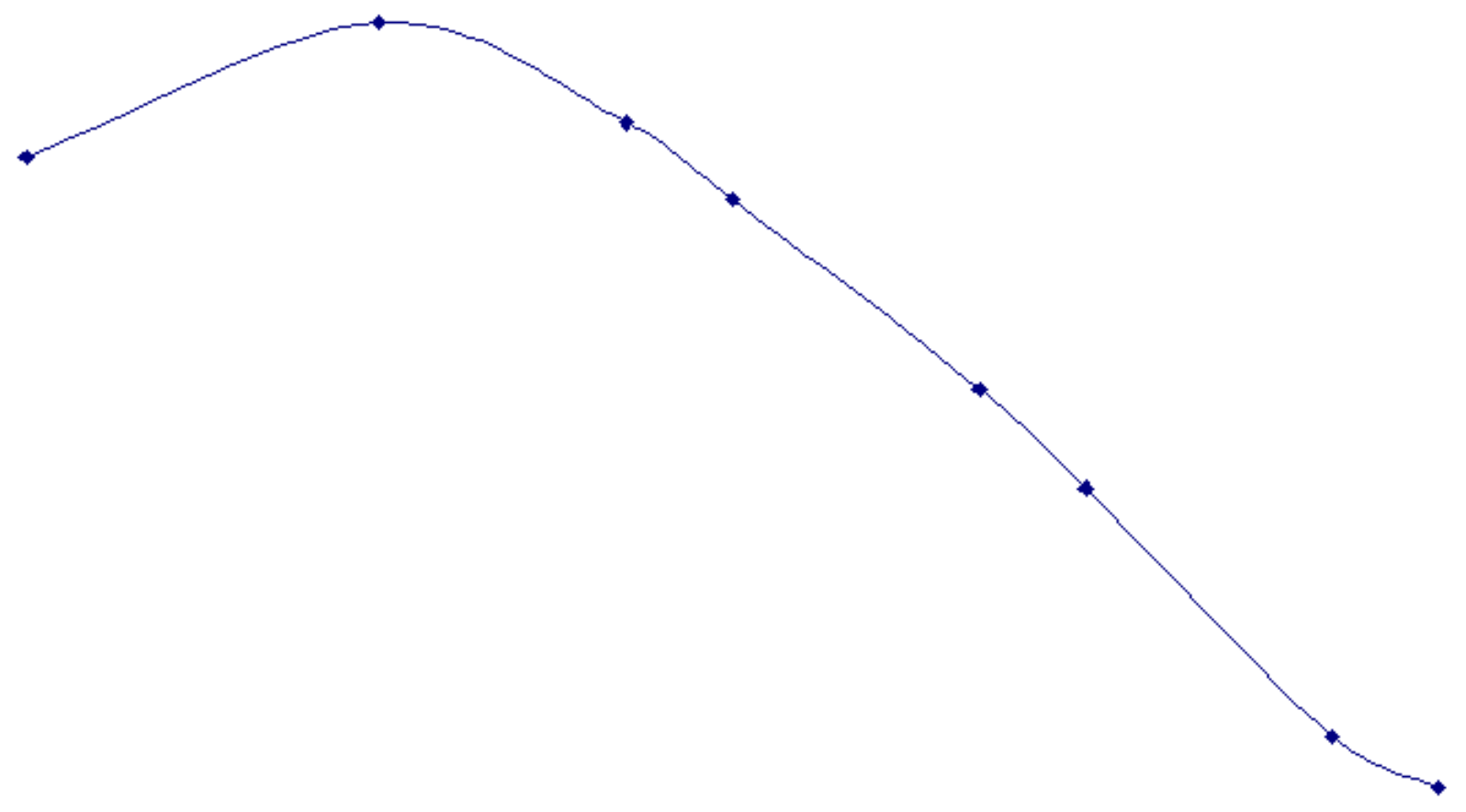


FY98 Annual Report

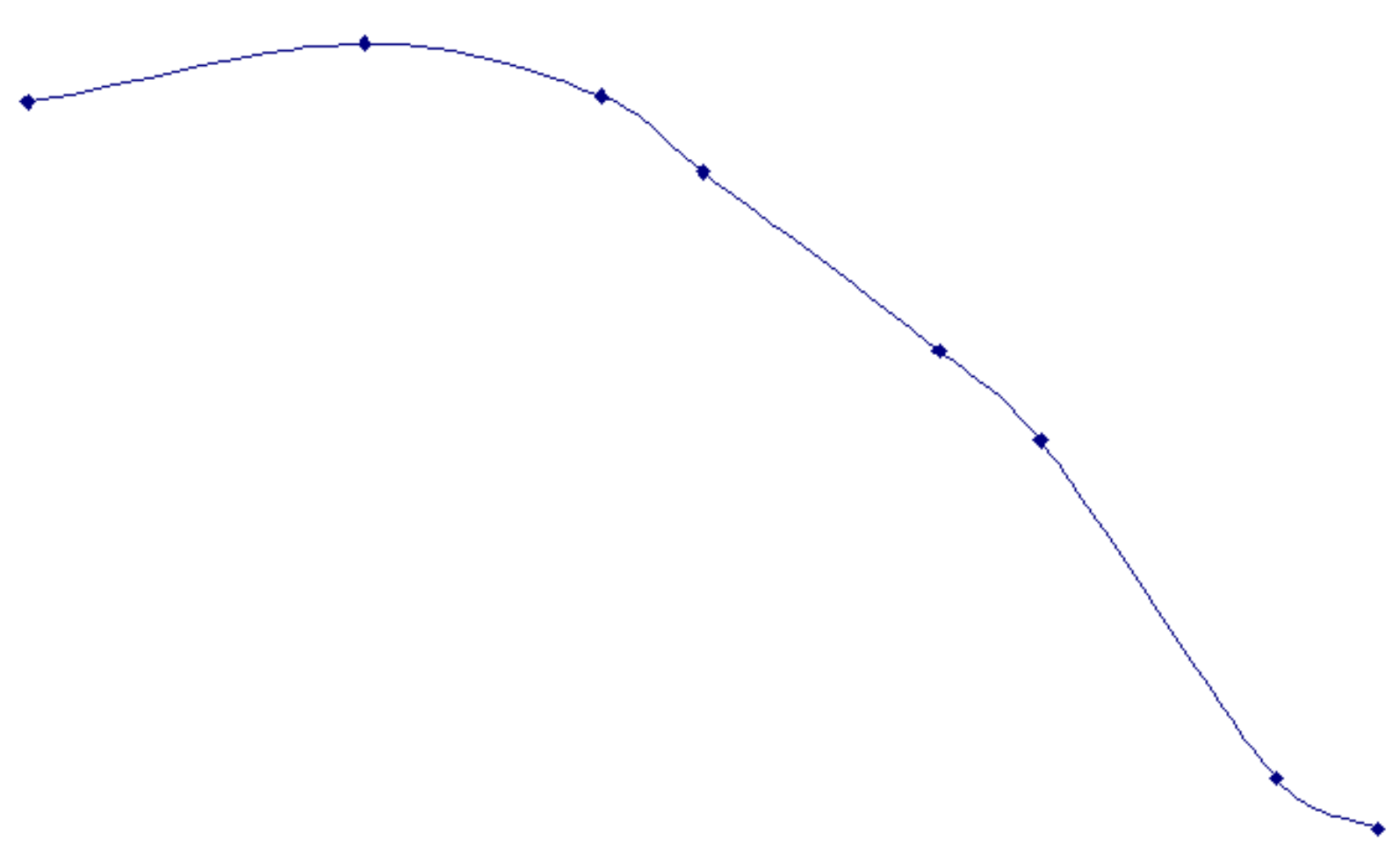

file://C//ManualConversion/HTML/6792benk.htm (218 of 250)4/9/2004 4:05:25 AM 


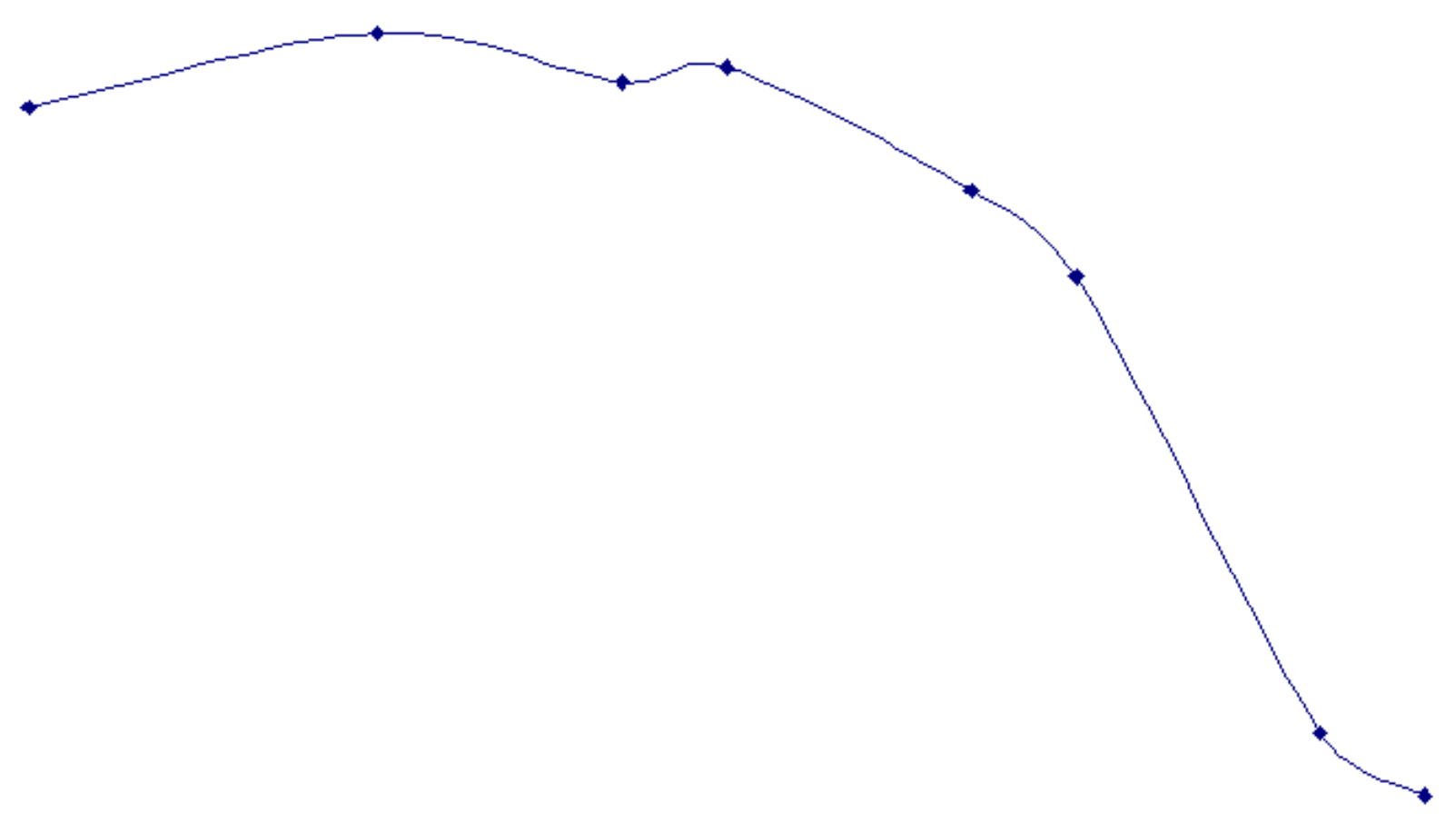


<smiles>CCCCC</smiles> 


\section{Appendix Q}

\section{Calibration Curves for Velva Sheen Mop Oil} on Aluminum Panels and Stainless Steel Disks

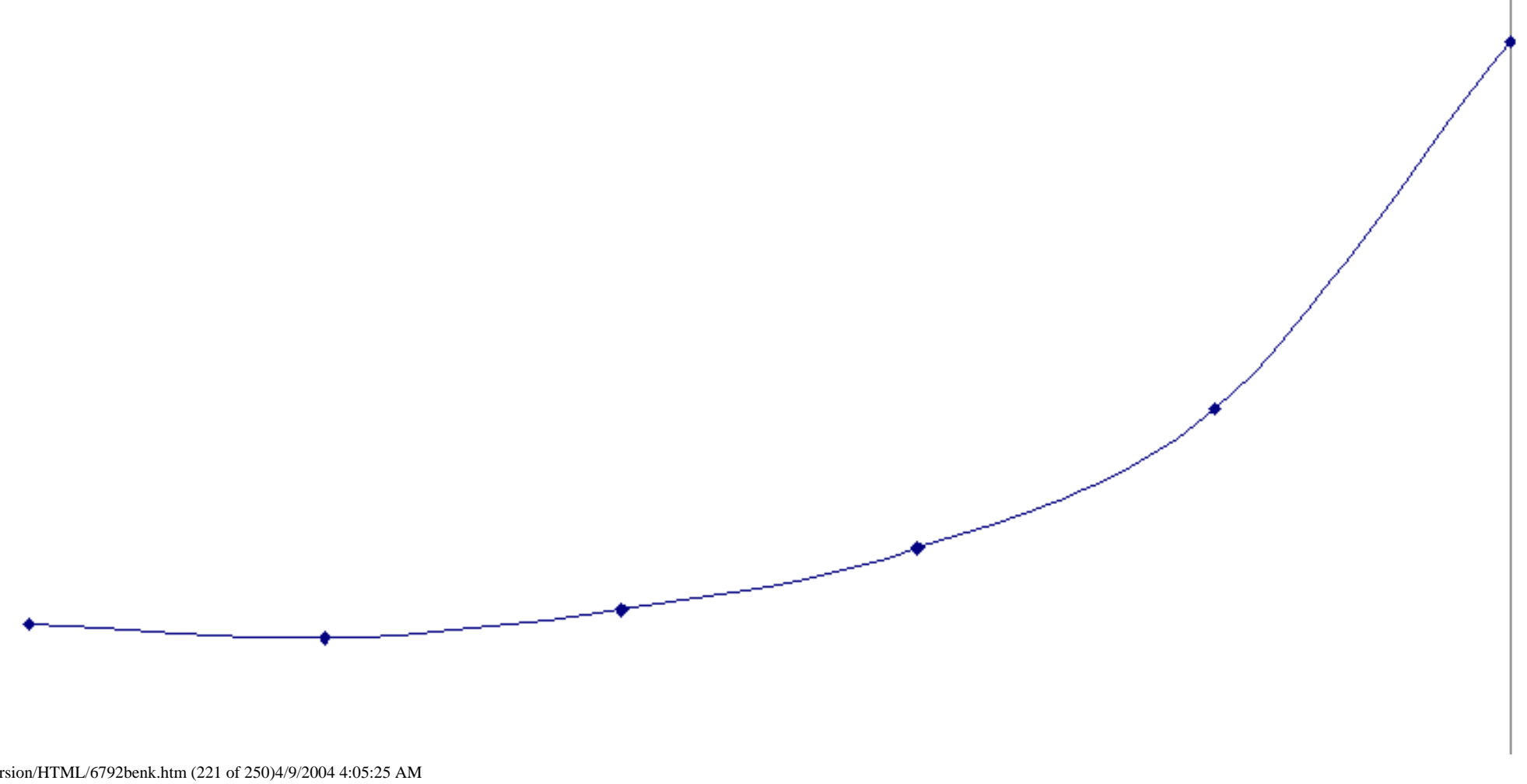


FY98 Annual Report

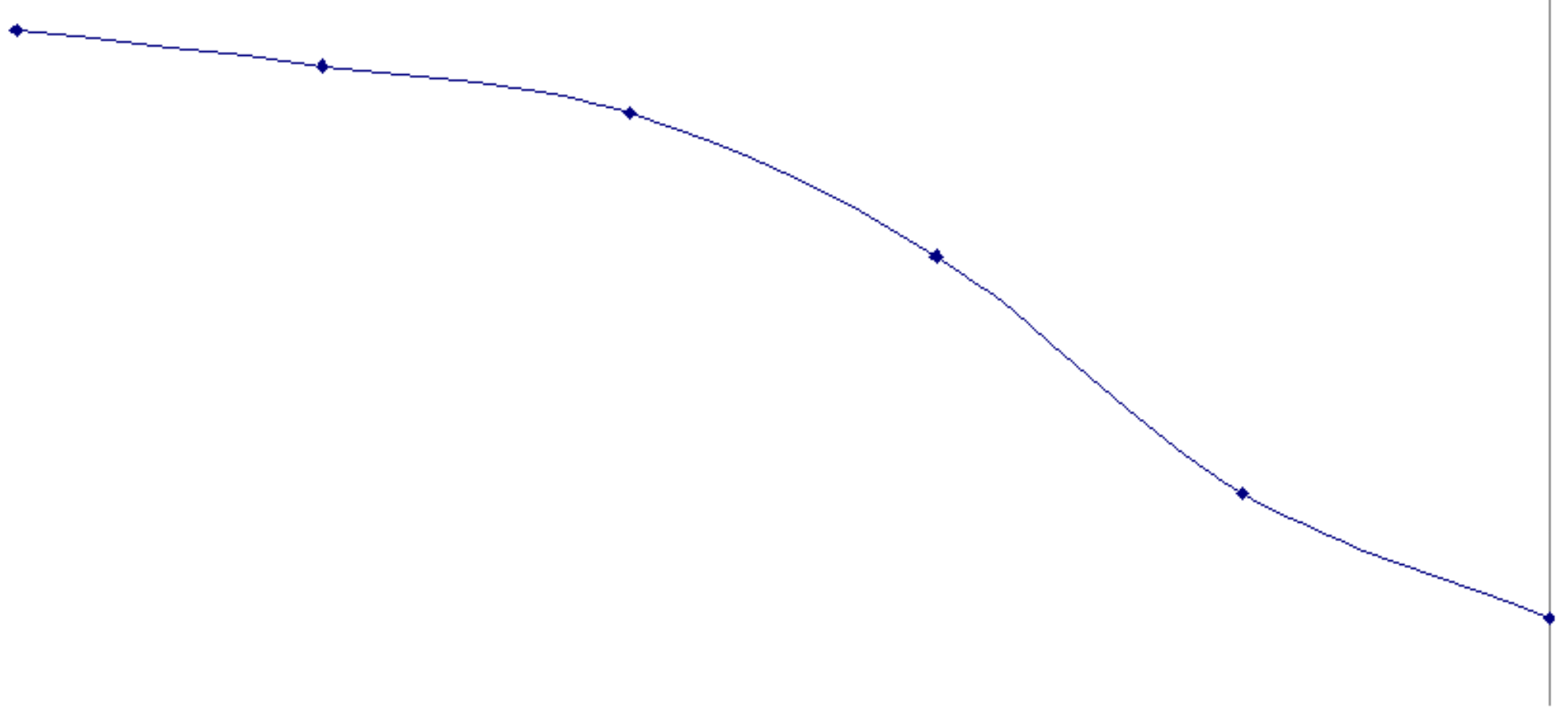

file://C//ManualConversion/HTML/6792benk.htm (222 of 250)4/9/2004 4:05:25 AM 

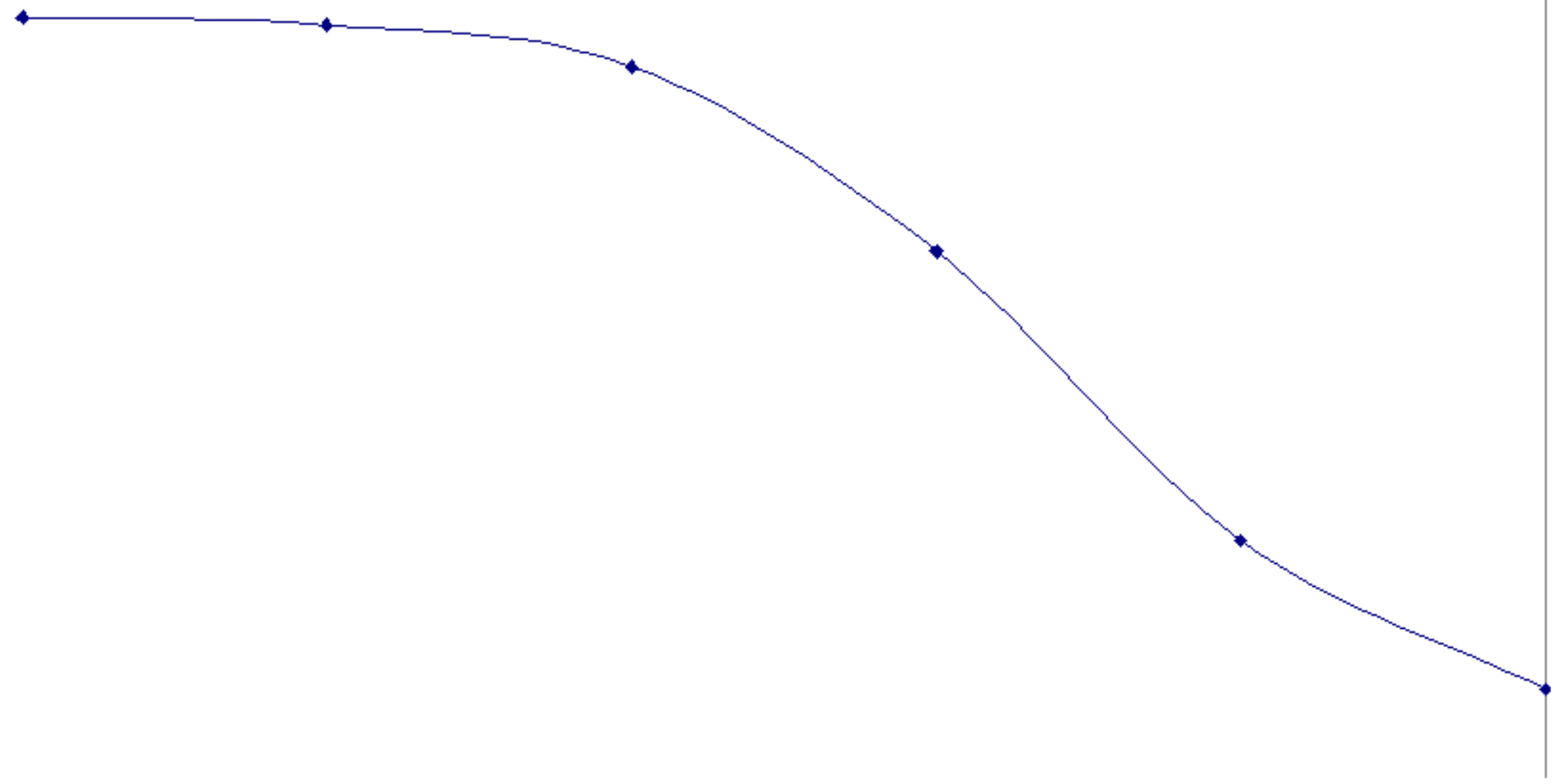


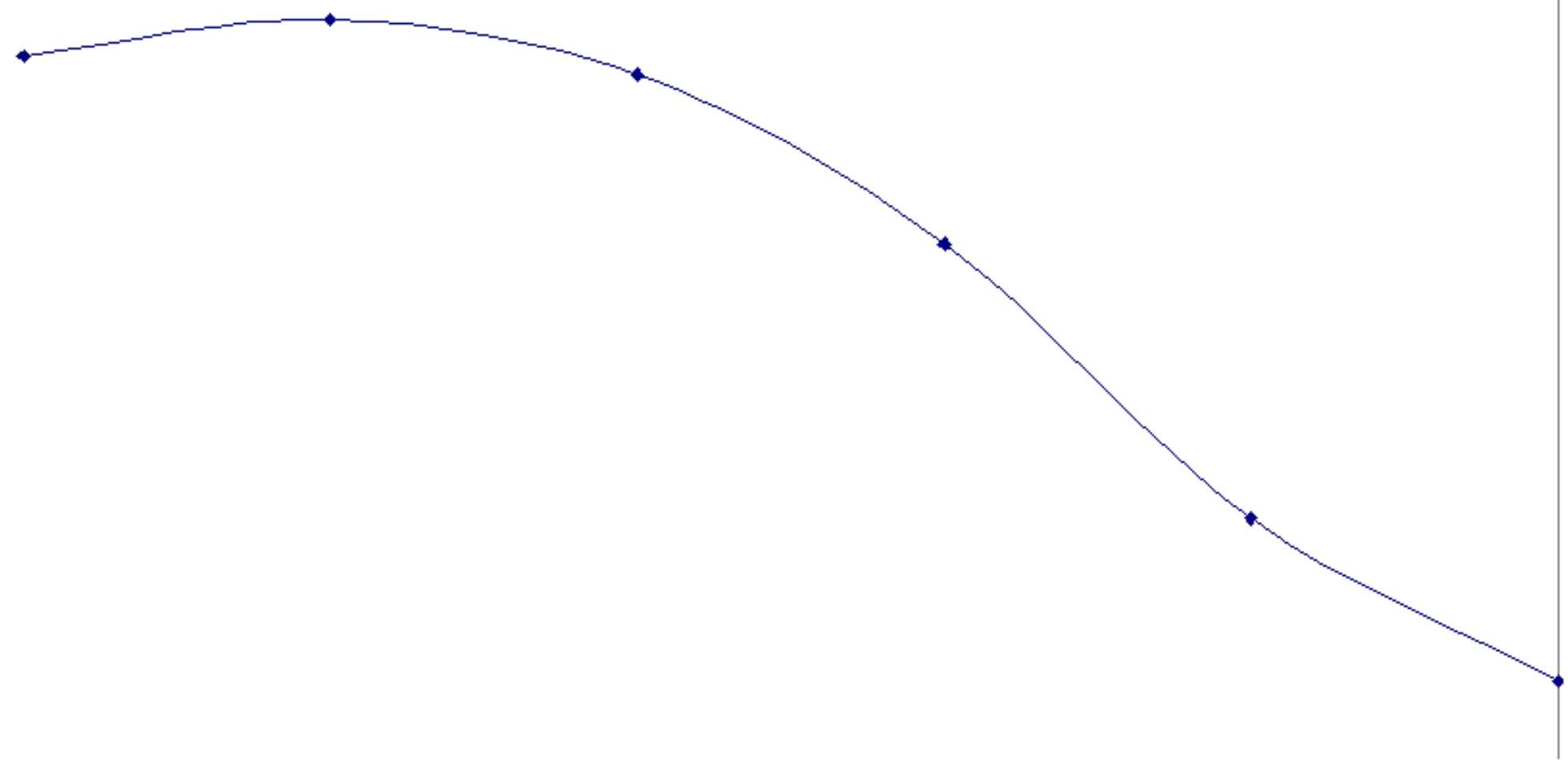




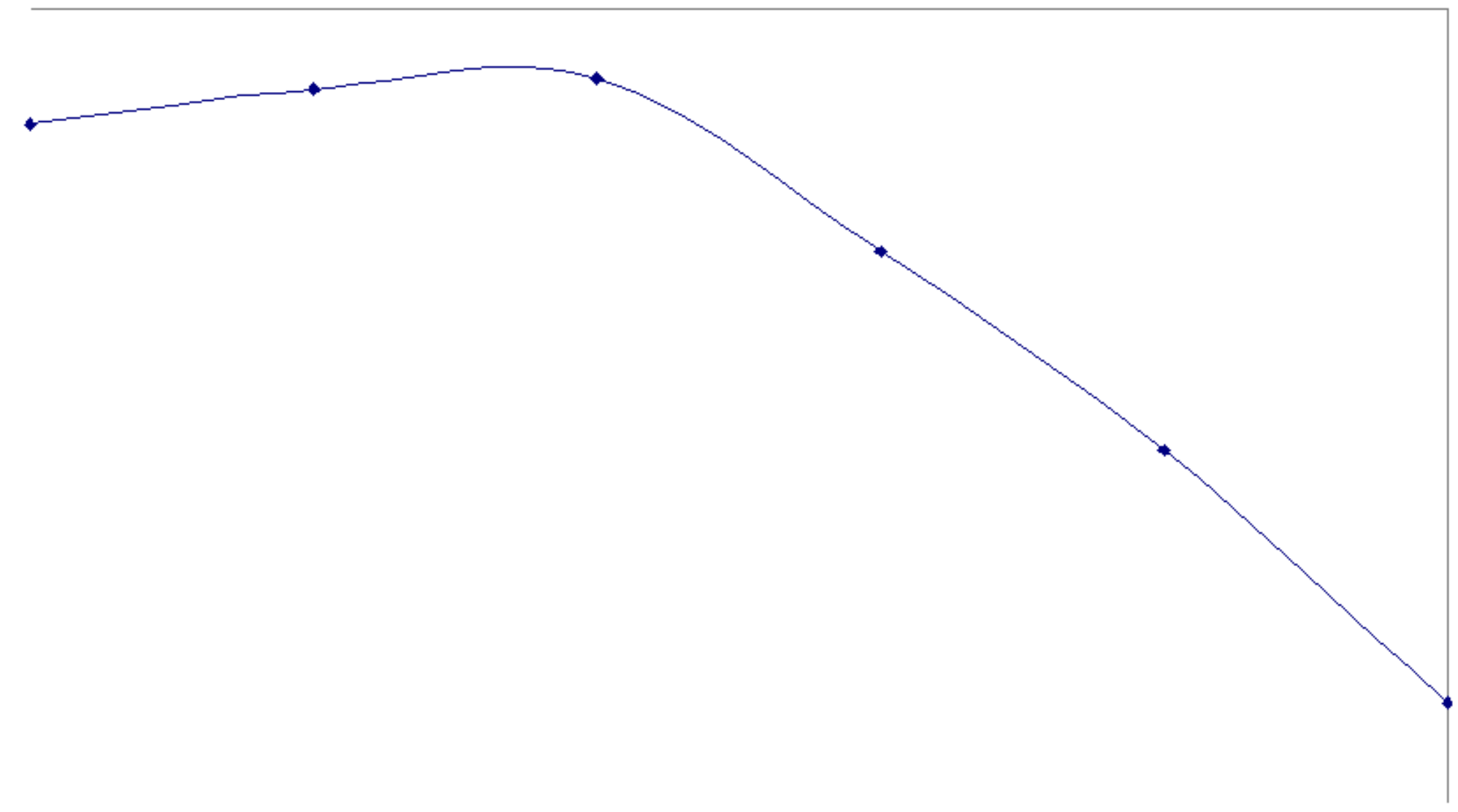




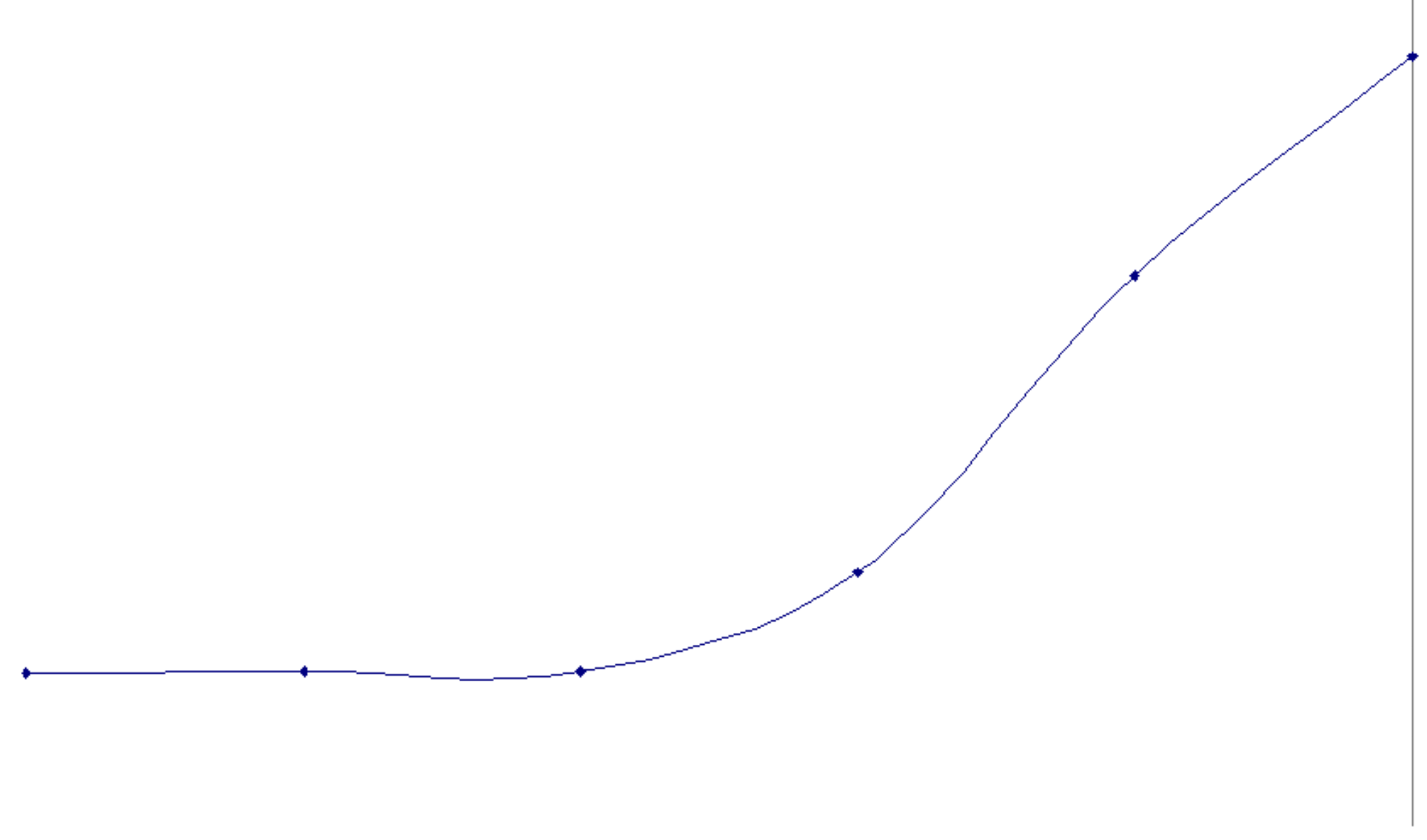



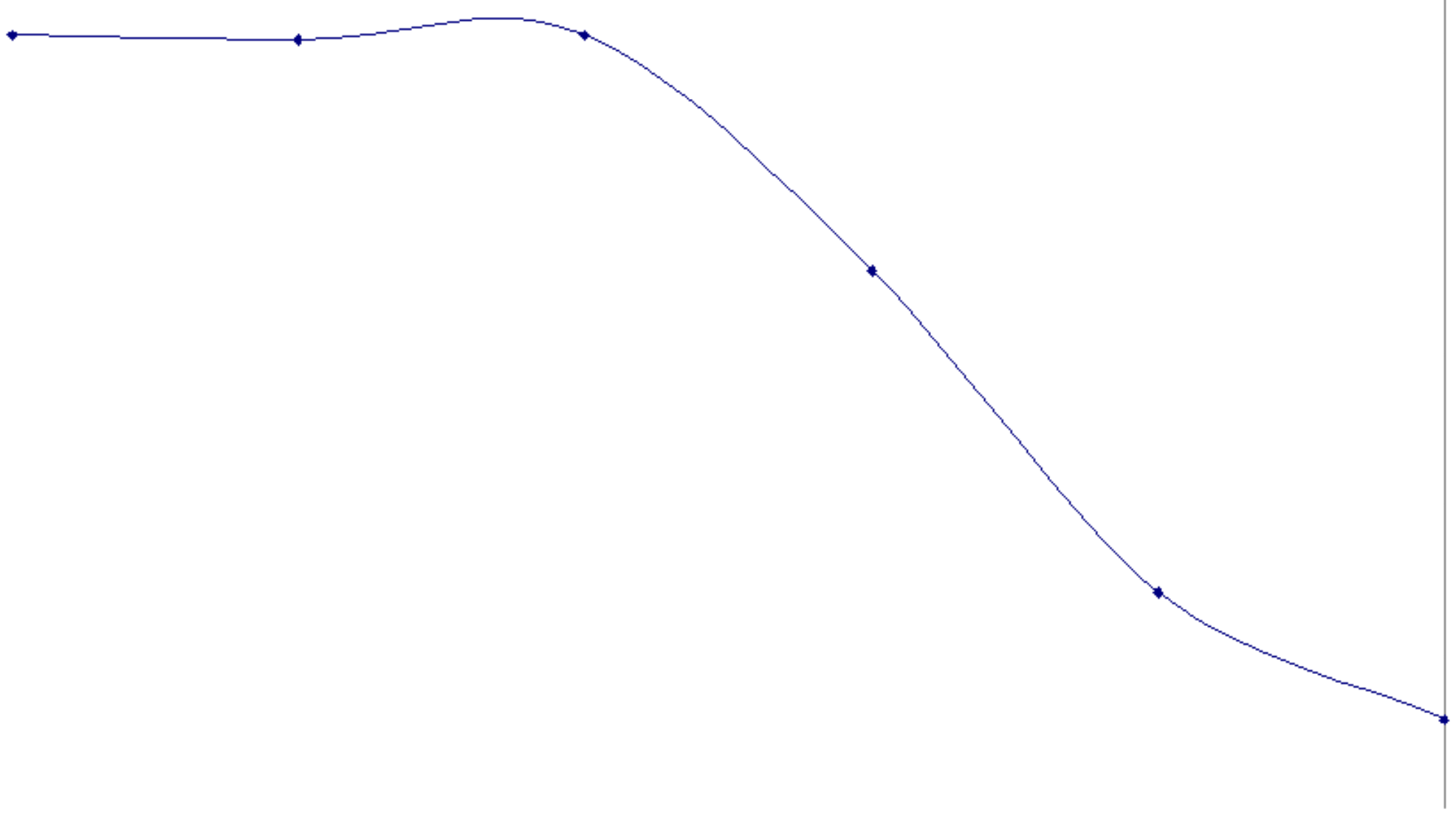
<smiles>CCCCC</smiles> 

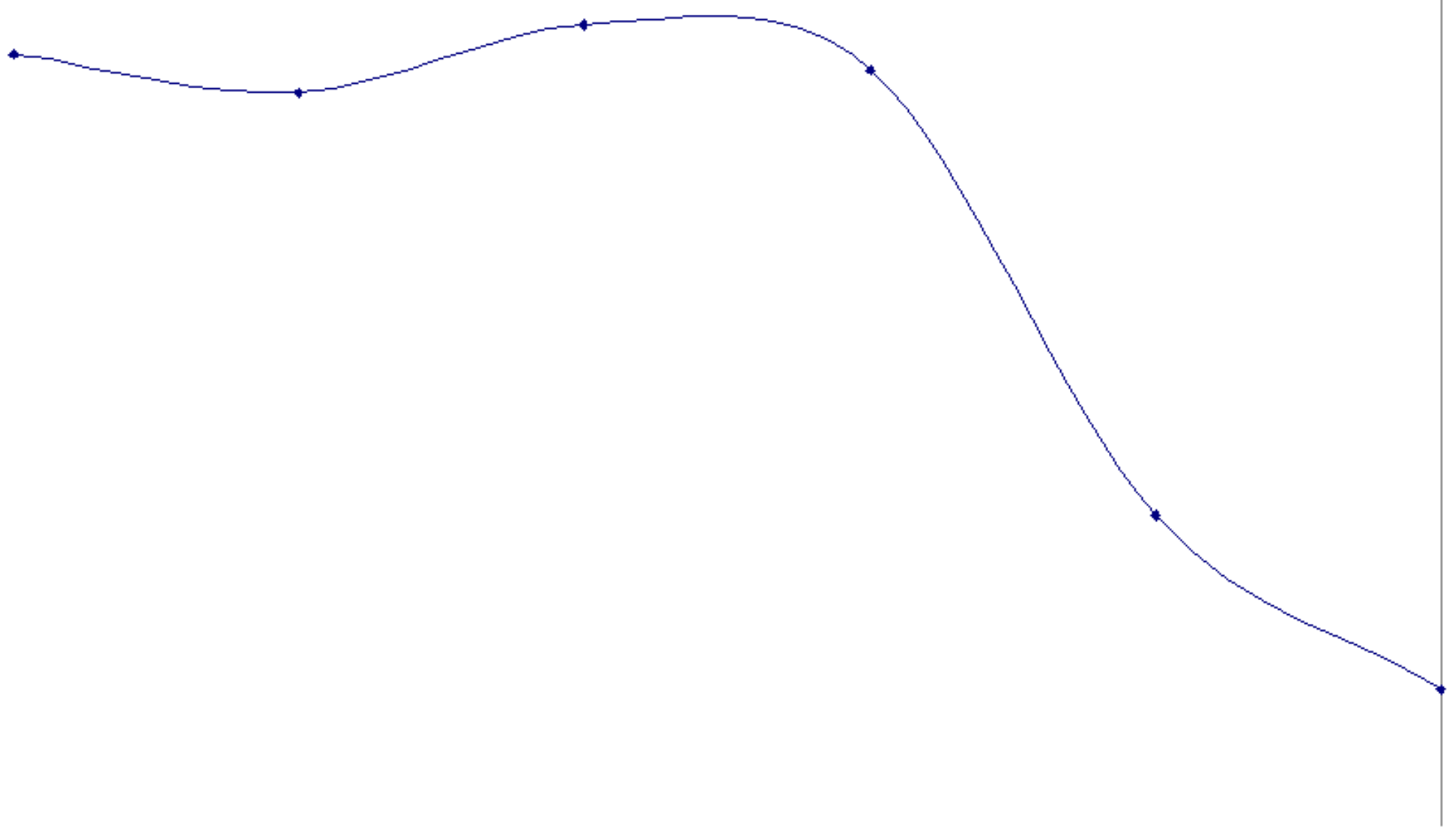


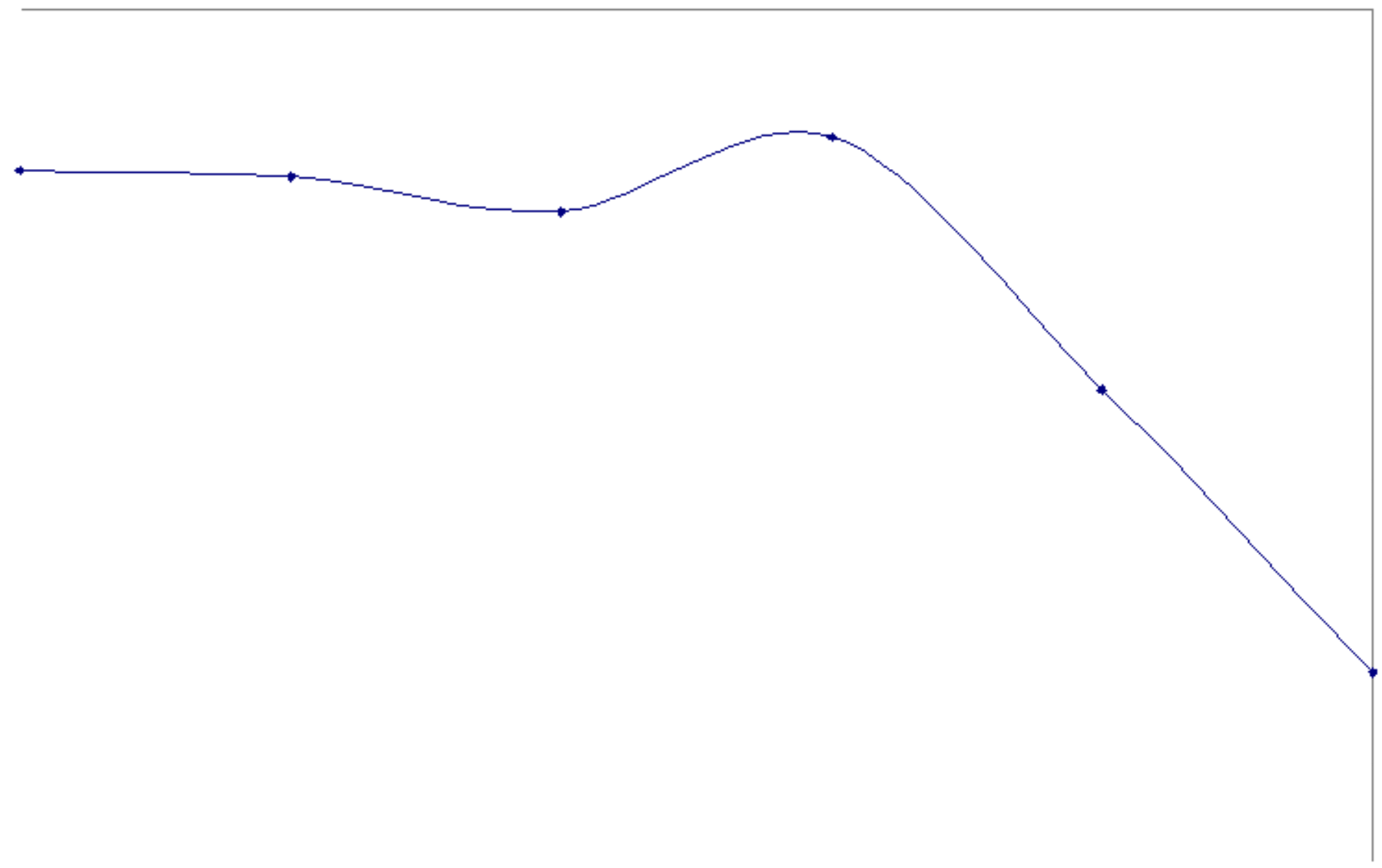




\section{Appendix R}

\section{Calibration Curves for Regal R\&O 32 Oil}

on Aluminum Panels and Stainless Steel Disks

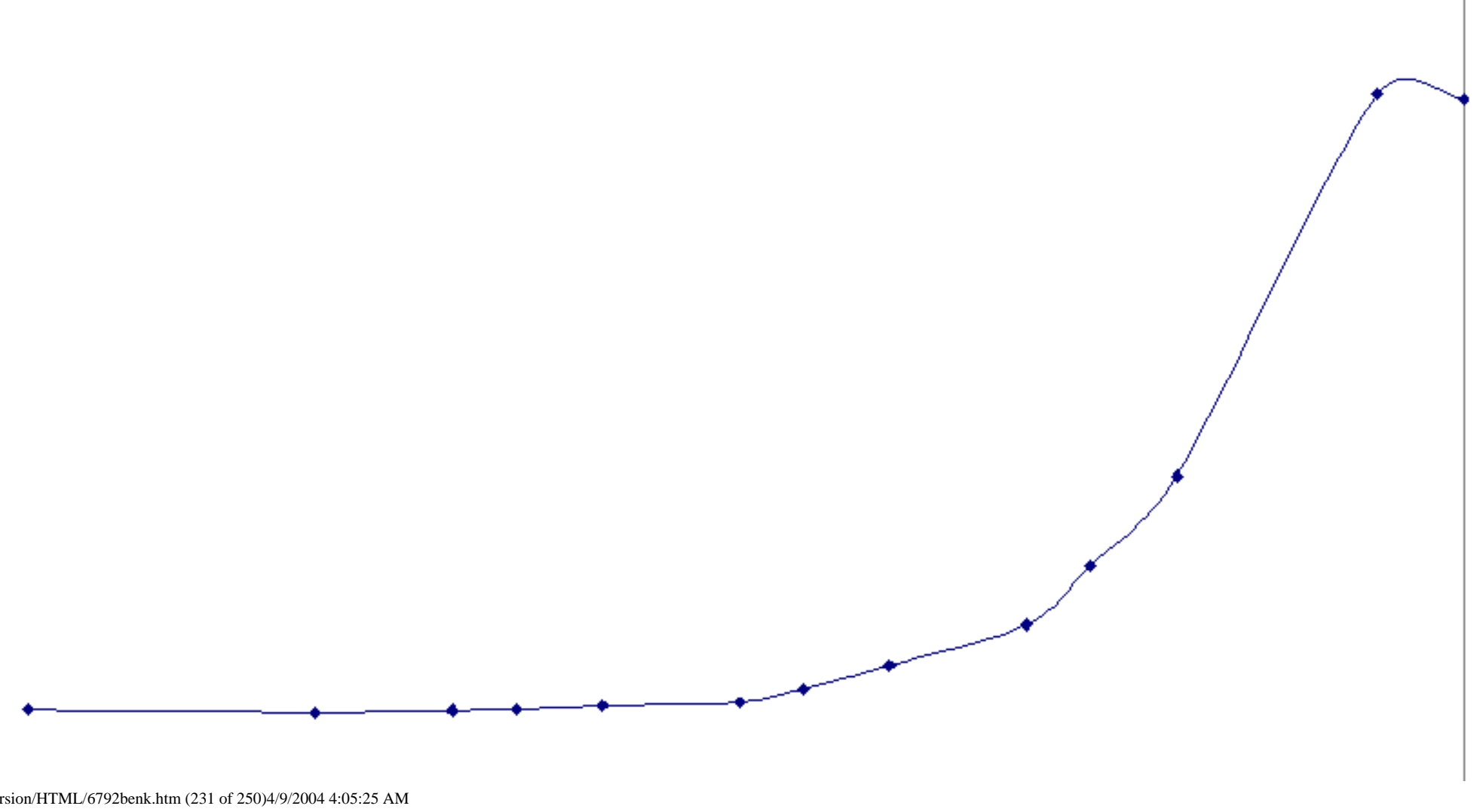


FY98 Annual Report

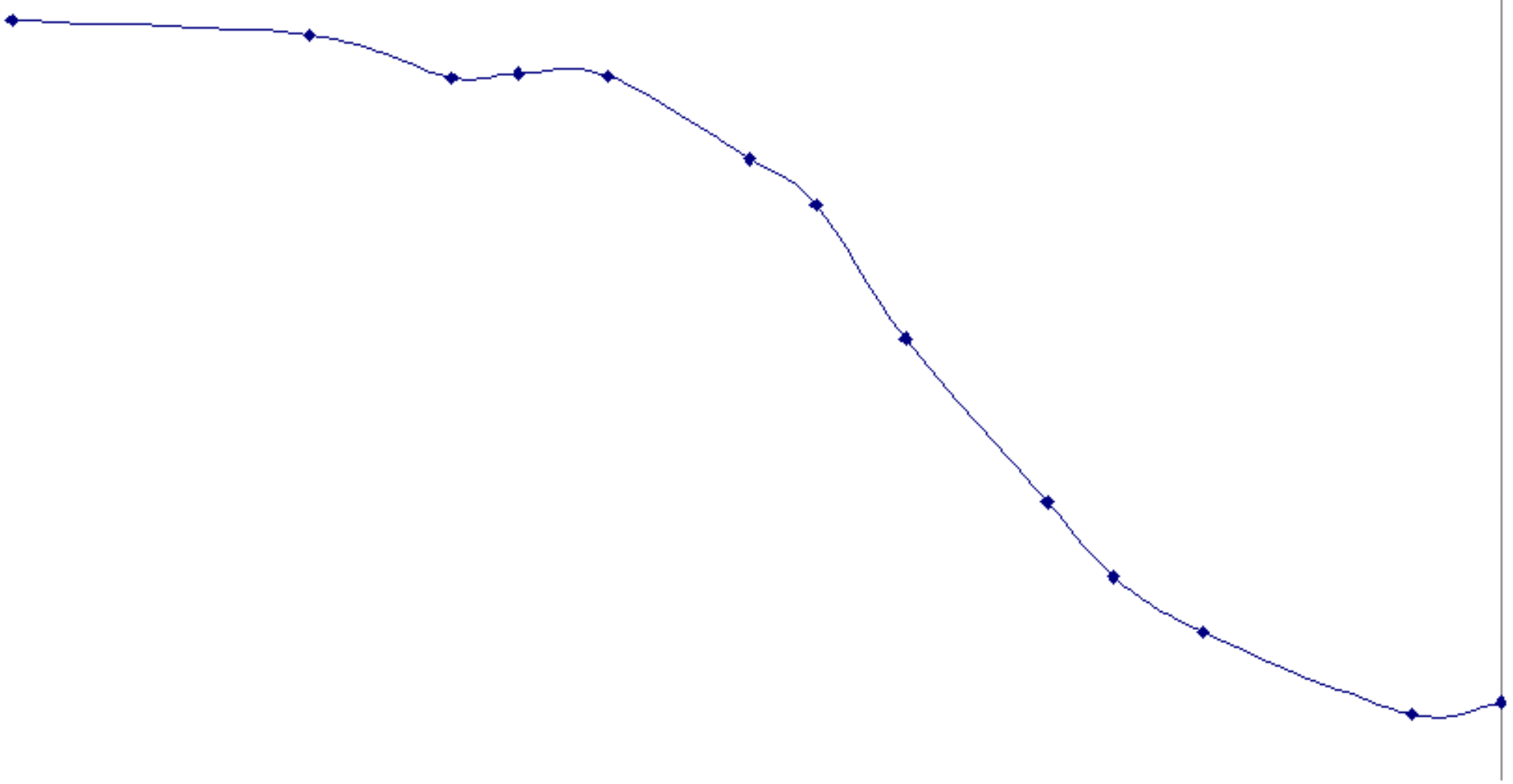

file://C|/ManualConversion/HTML/6792benk.htm (232 of 250)4/9/2004 4:05:25 AM 

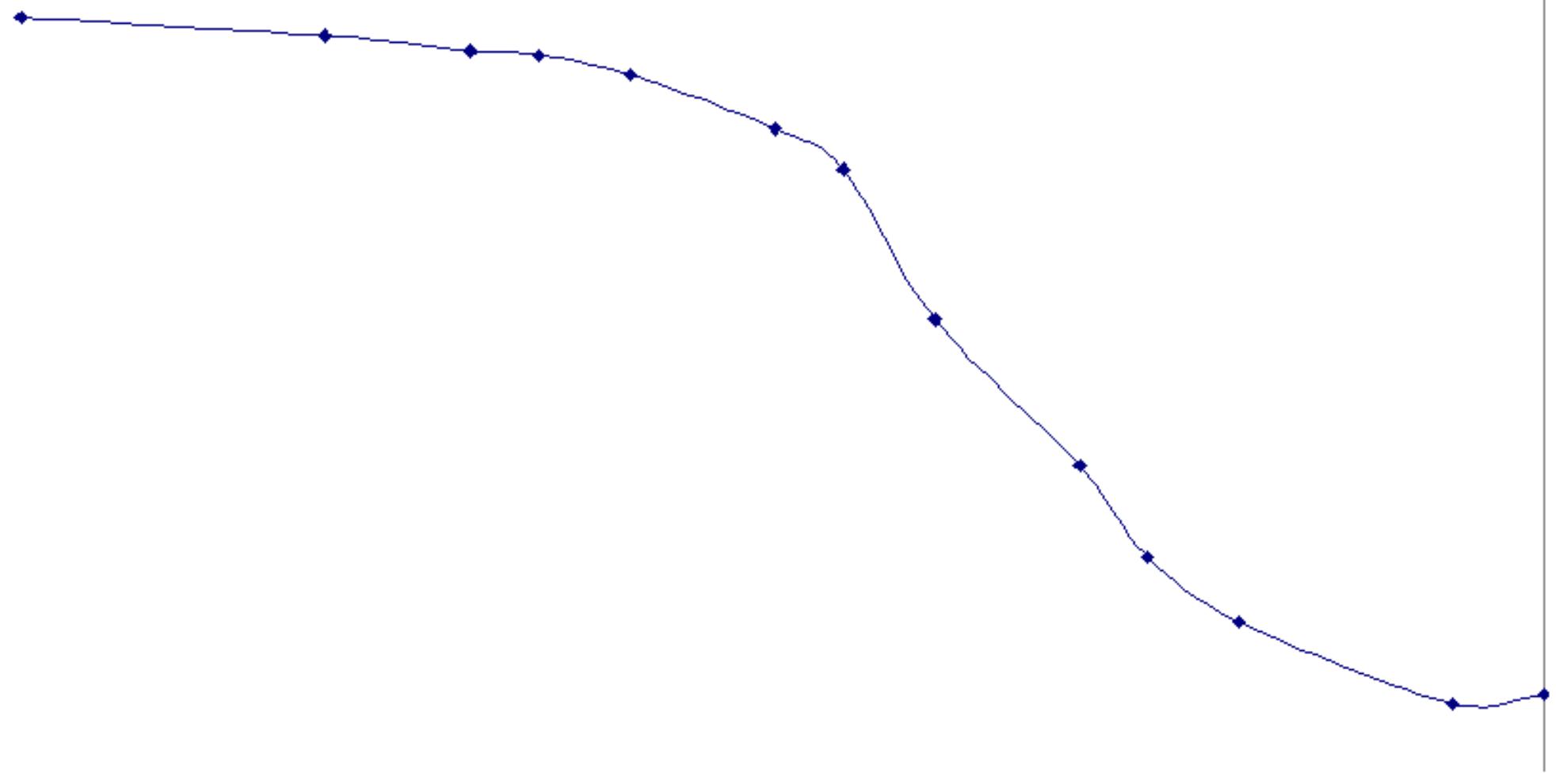


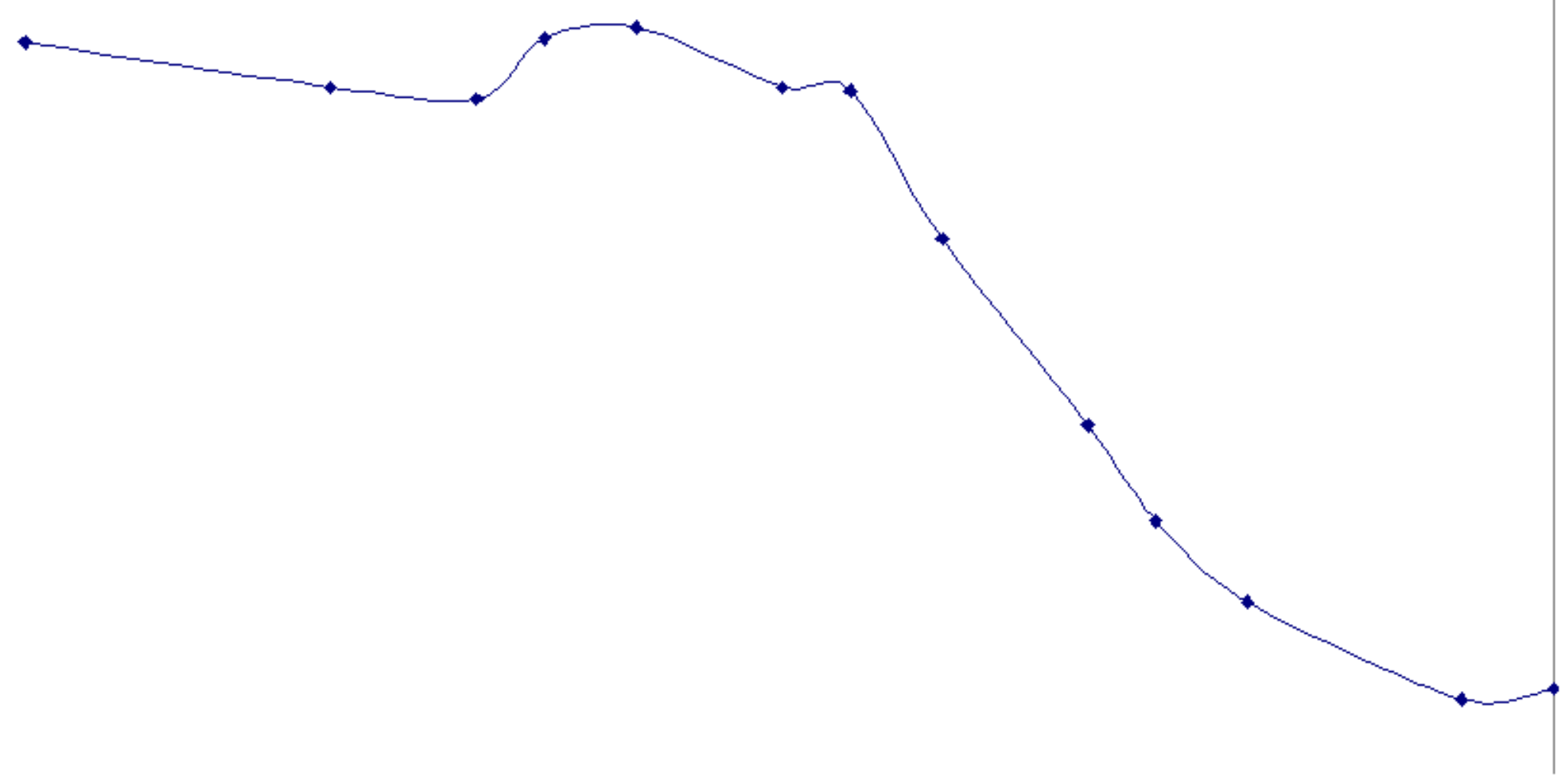




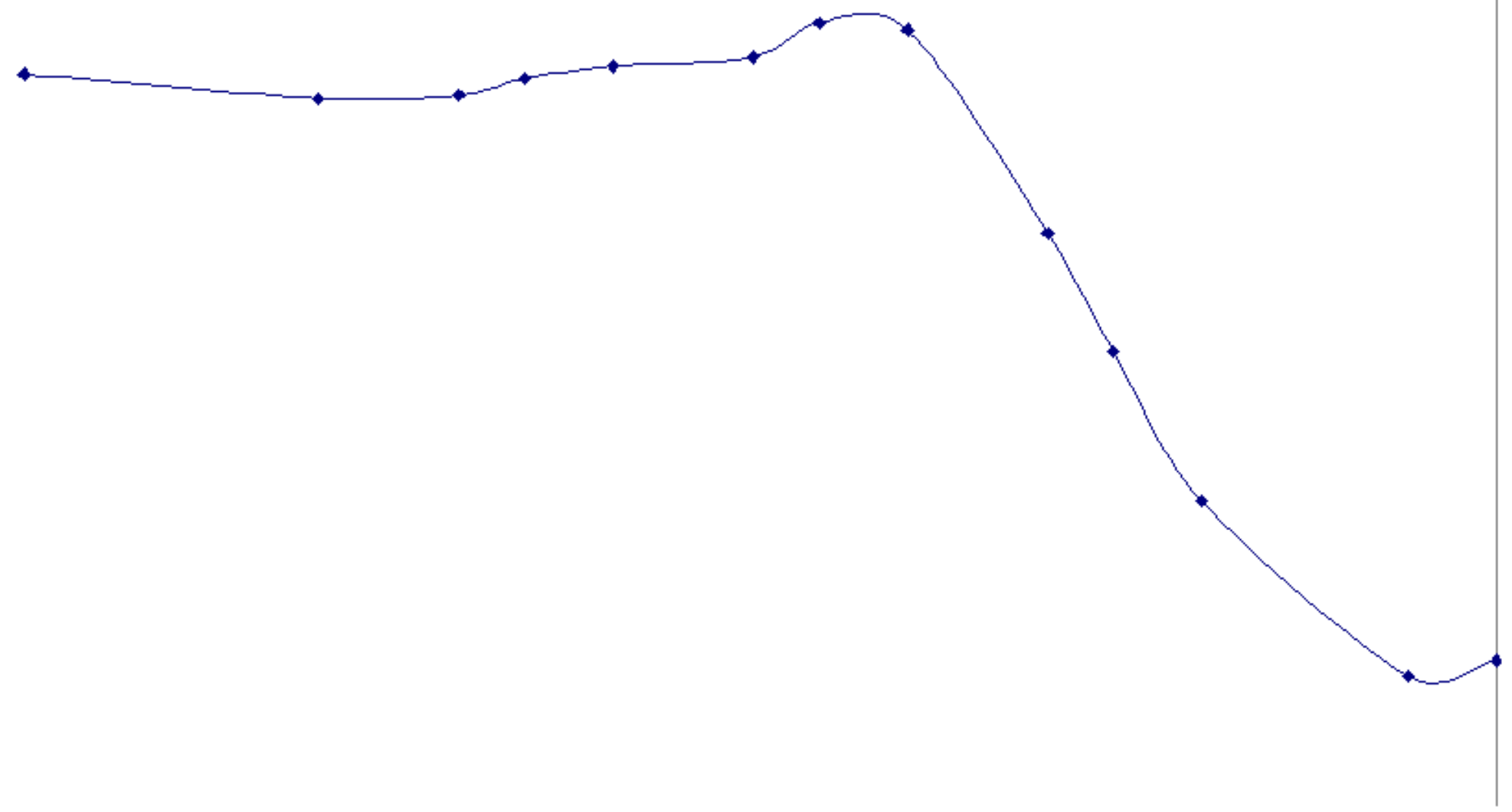


<smiles>CCCC</smiles> 
FY98 Annual Report

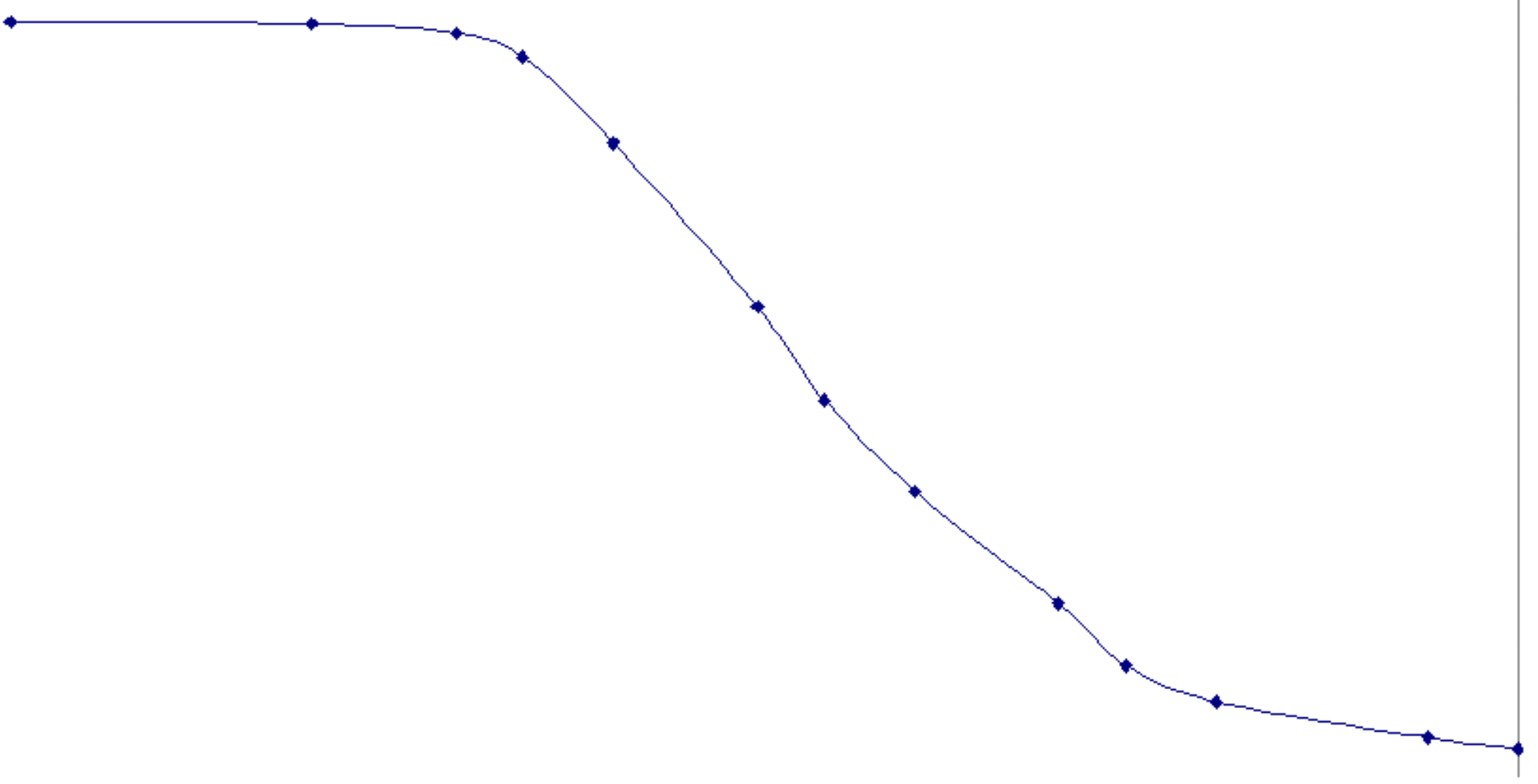

file://C//ManualConversion/HTML/6792benk.htm (237 of 250)4/9/2004 4:05:25 AM 


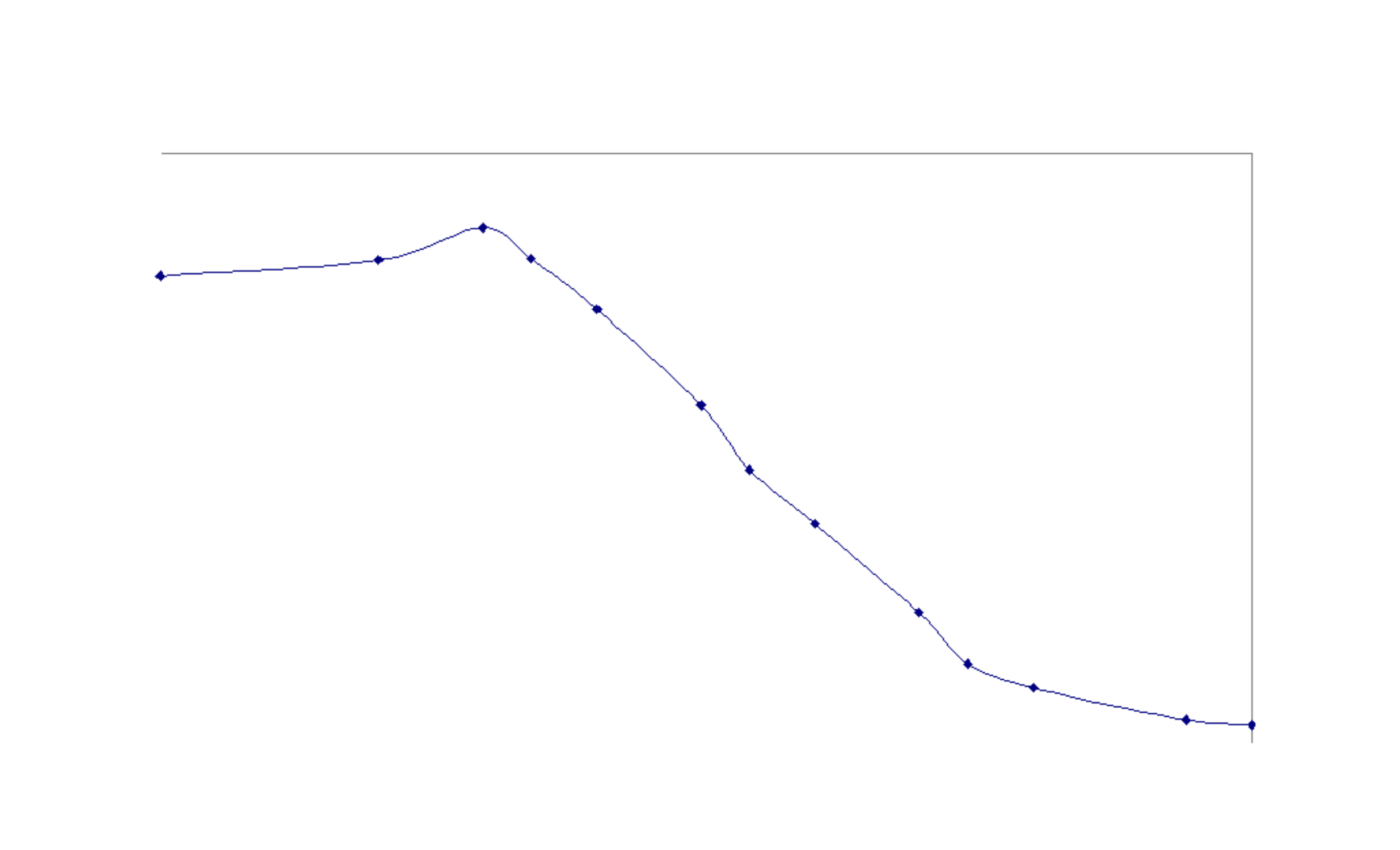


FY98 Annual Report

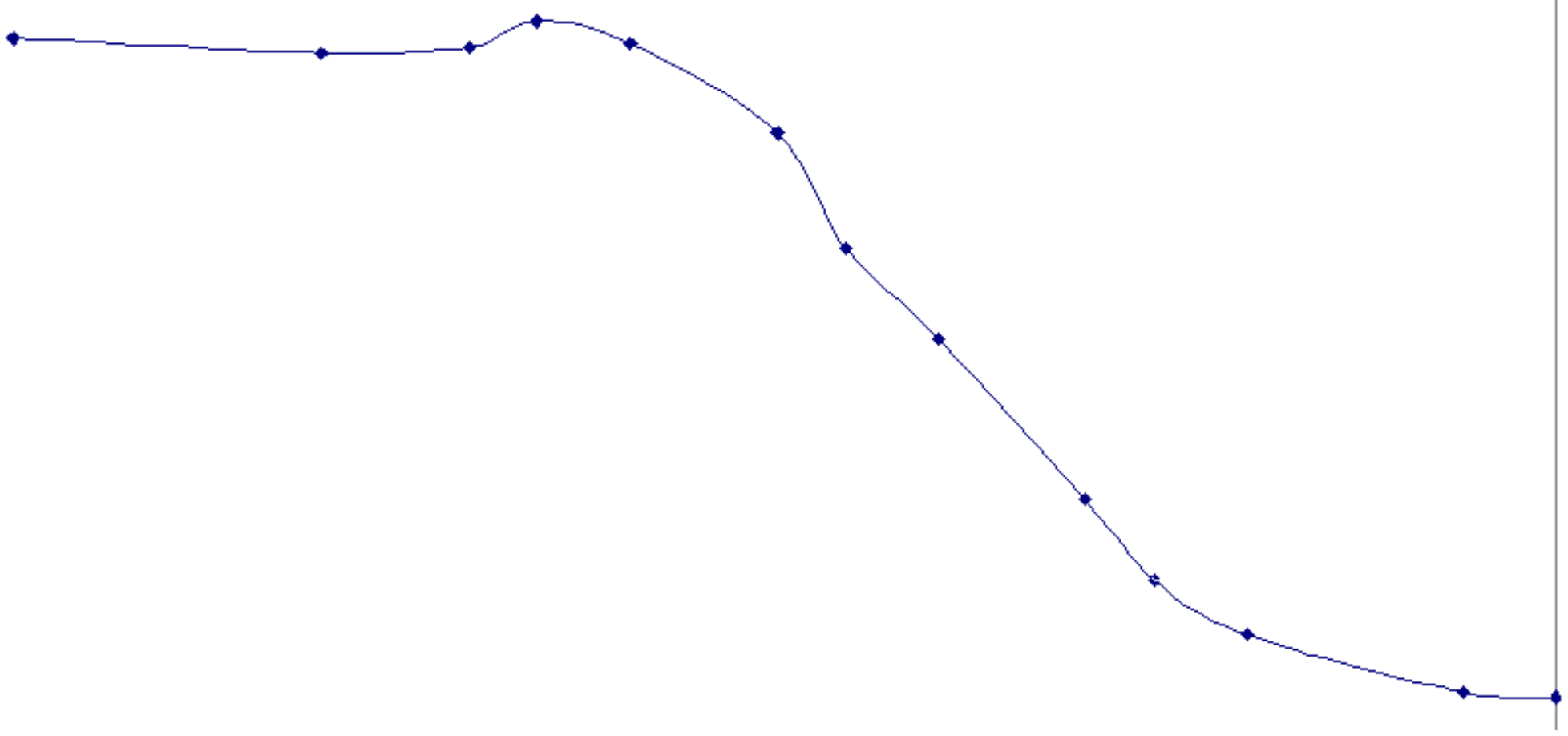

file://C|/ManualConversion/HTML/6792benk.htm (239 of 250)4/9/2004 4:05:25 AM 


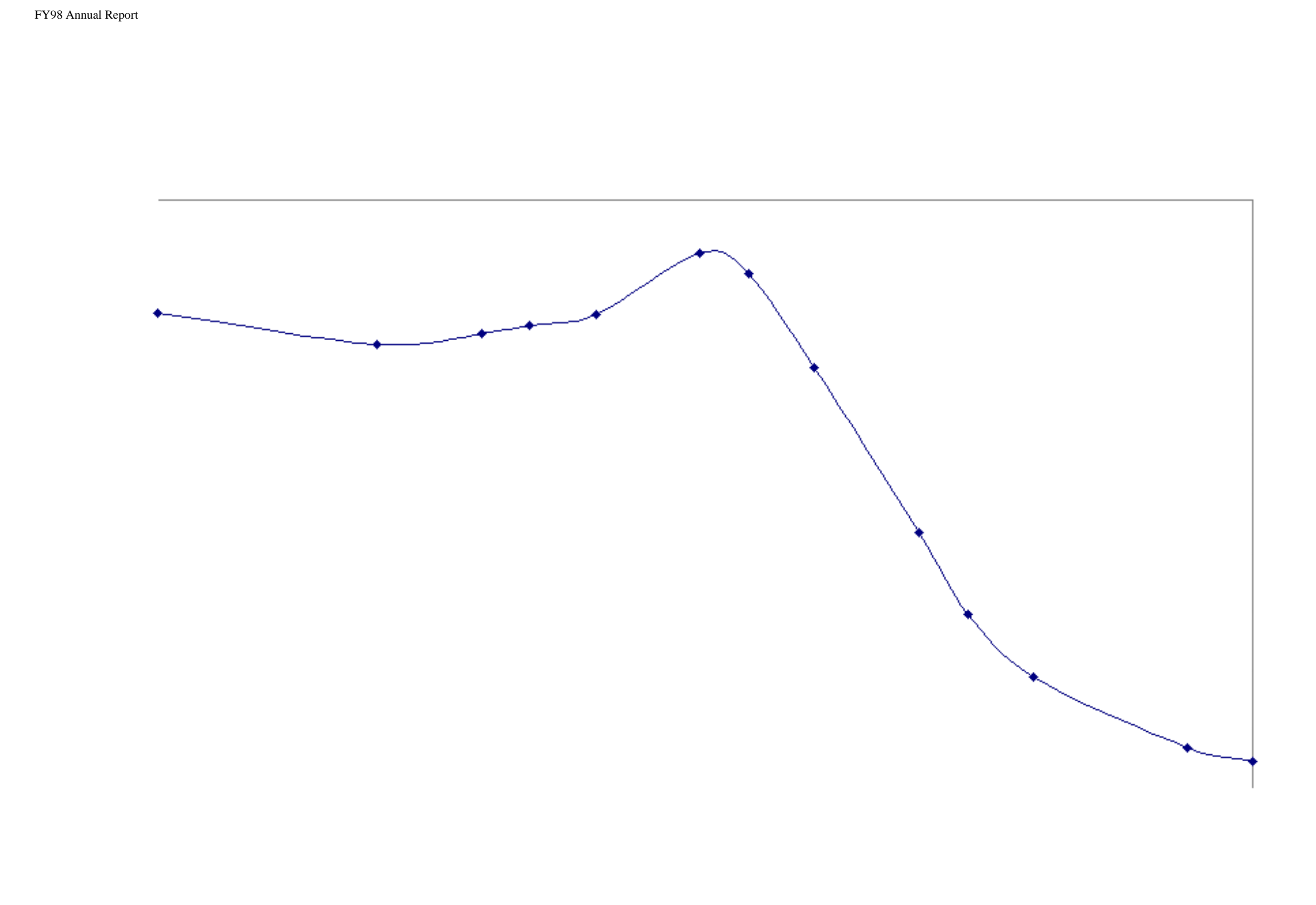




\section{Appendix S}

\section{MESERAN Data Plot and Calibration Curve}

for Hydrocarbon Blend

(70\% Pennex N47 and 30\% Hangsterfers Hard Cut \#511)

on Stainless Steel Disks

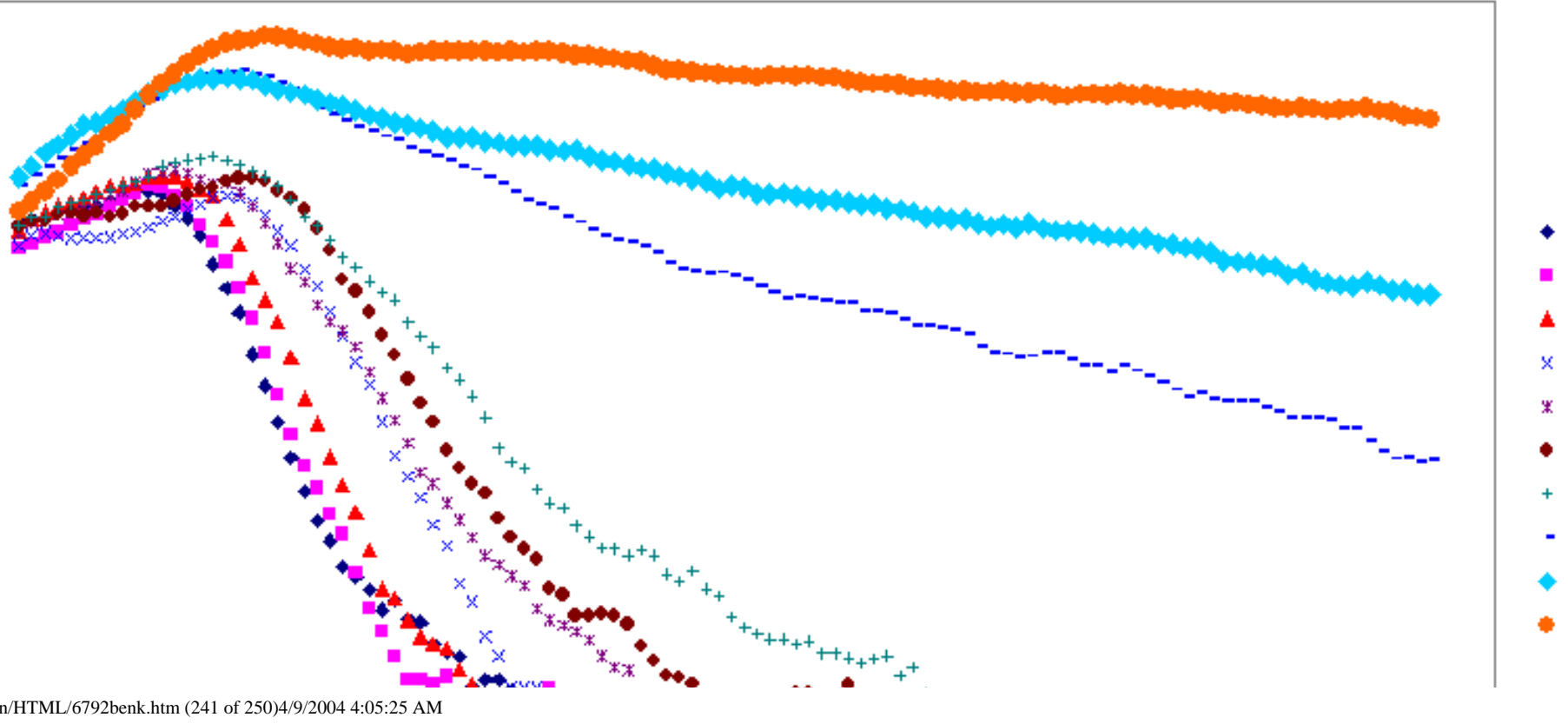




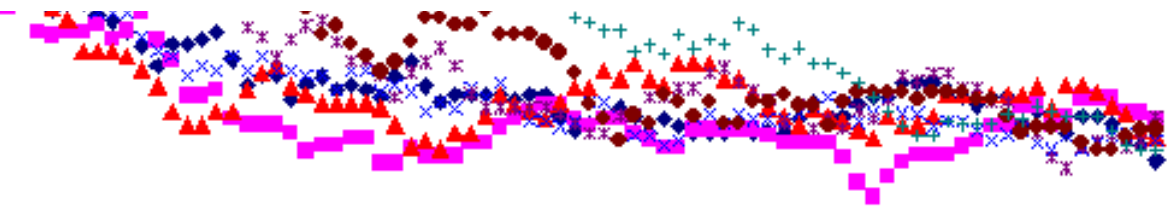

MESERAN Low Variance Slope Calibration of Hydrocarbon Blend (70\% Pennex N47 and 30\% Hangsterfers Hard Cut \#511) on Stainless Steel Disks Tested with BK

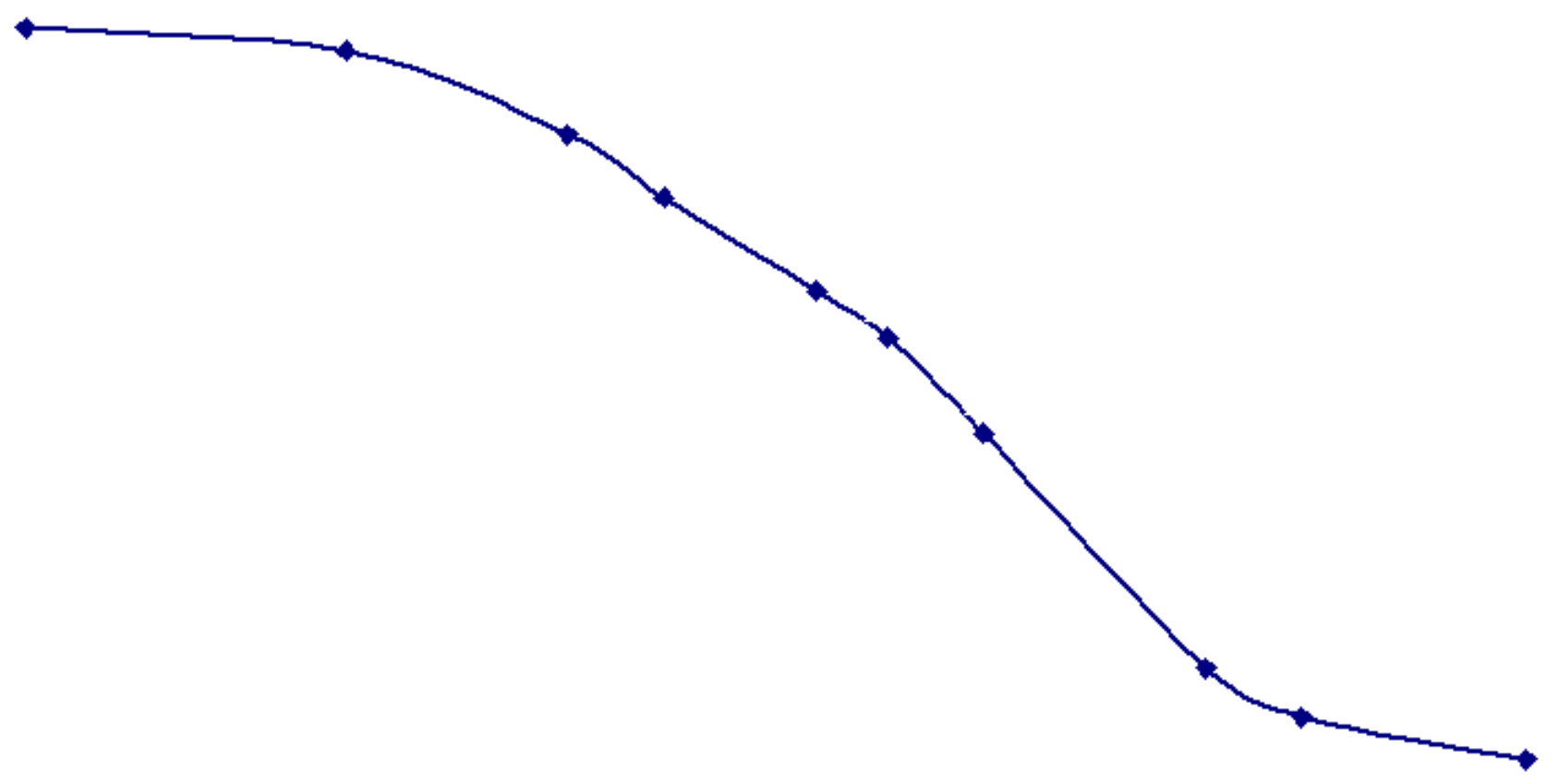


Appendix T

Calibration Curves for Mineral Oil on Stainless Steel Disks 
FY98 Annual Report

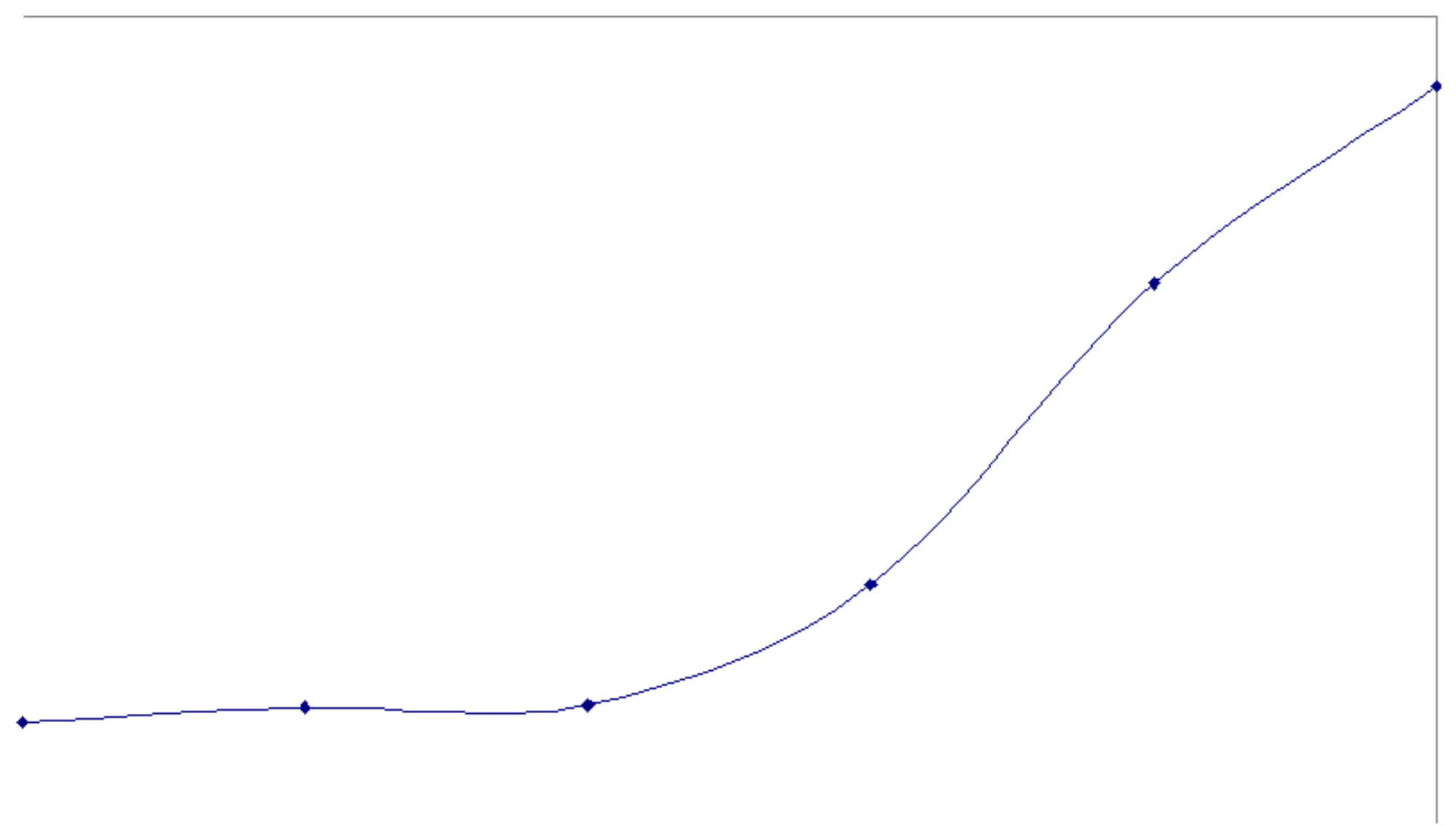

file:///C//ManualConversion/HTML/6792benk.htm (244 of 250)4/9/2004 4:05:25 AM 


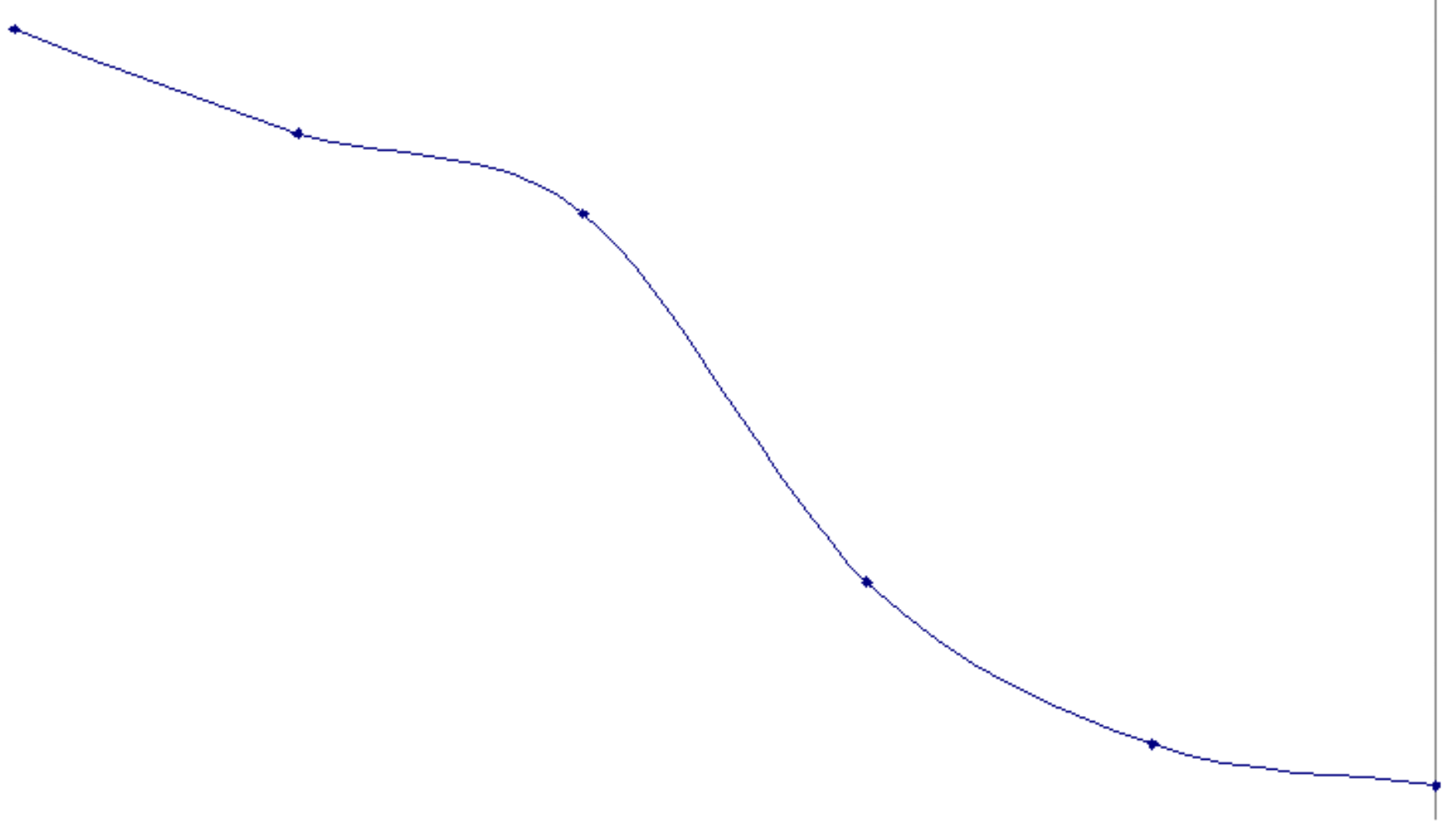


Appendix U

Calibration Curves for Hexatriacontane on Stainless Steel Disks

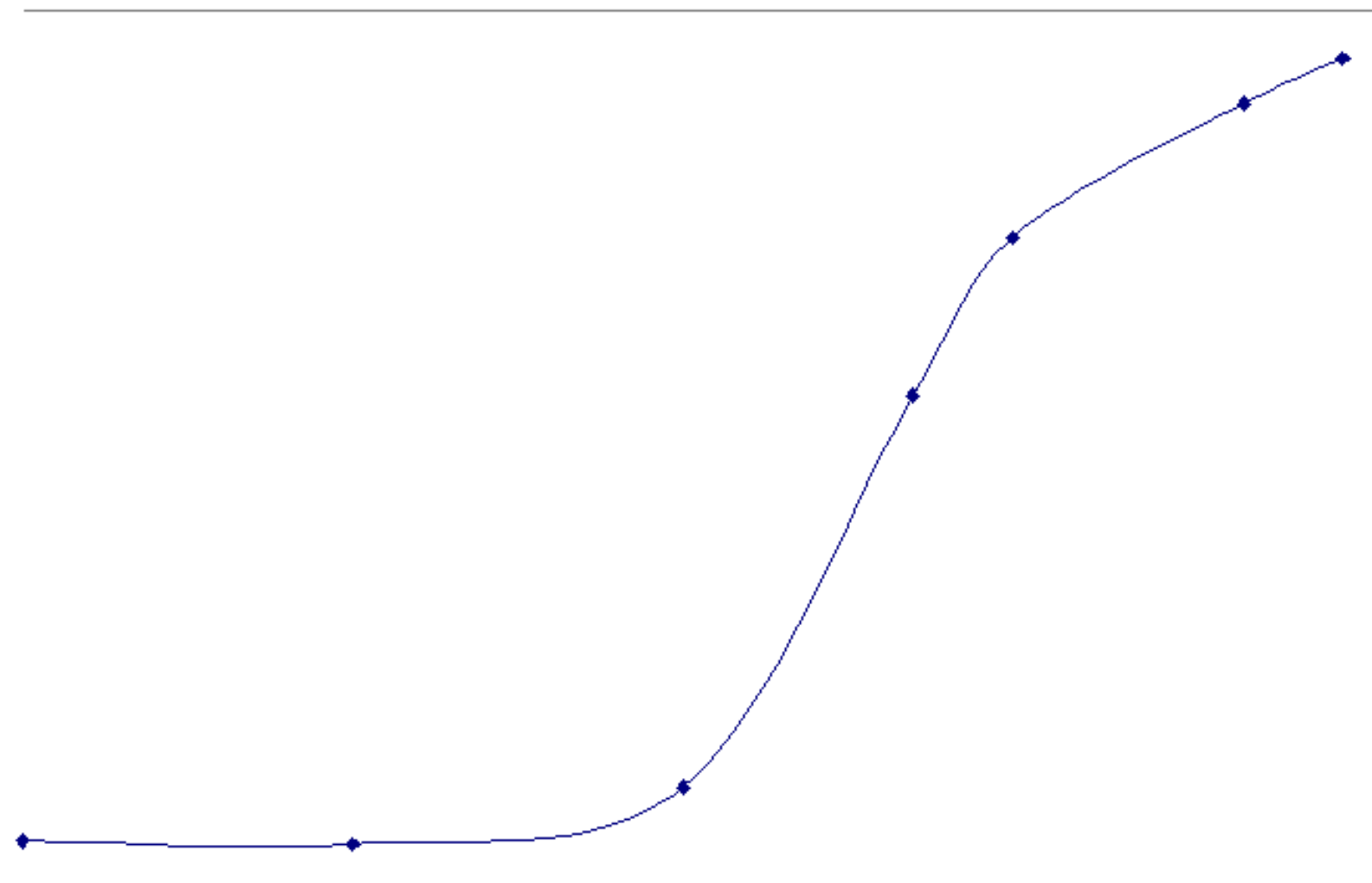



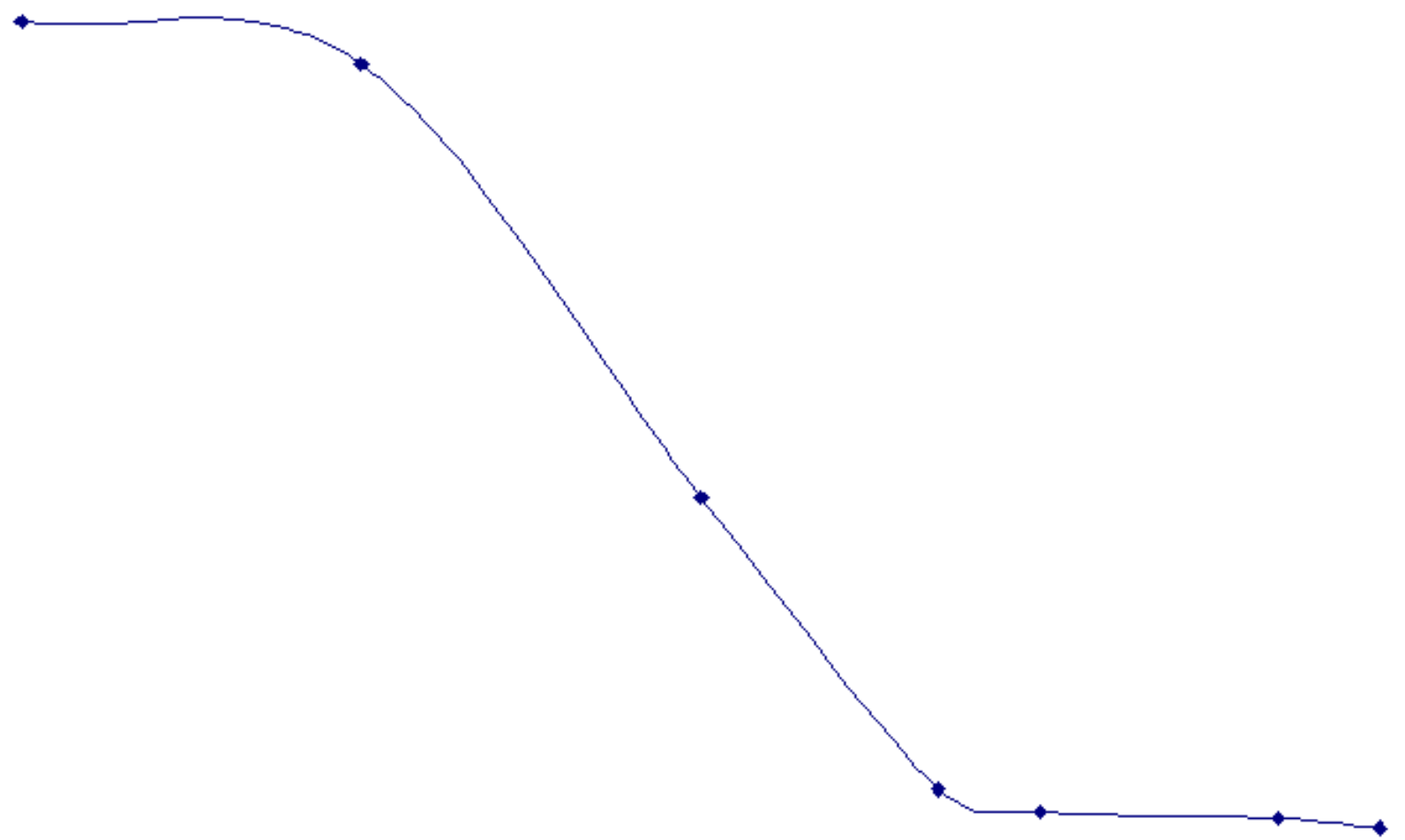


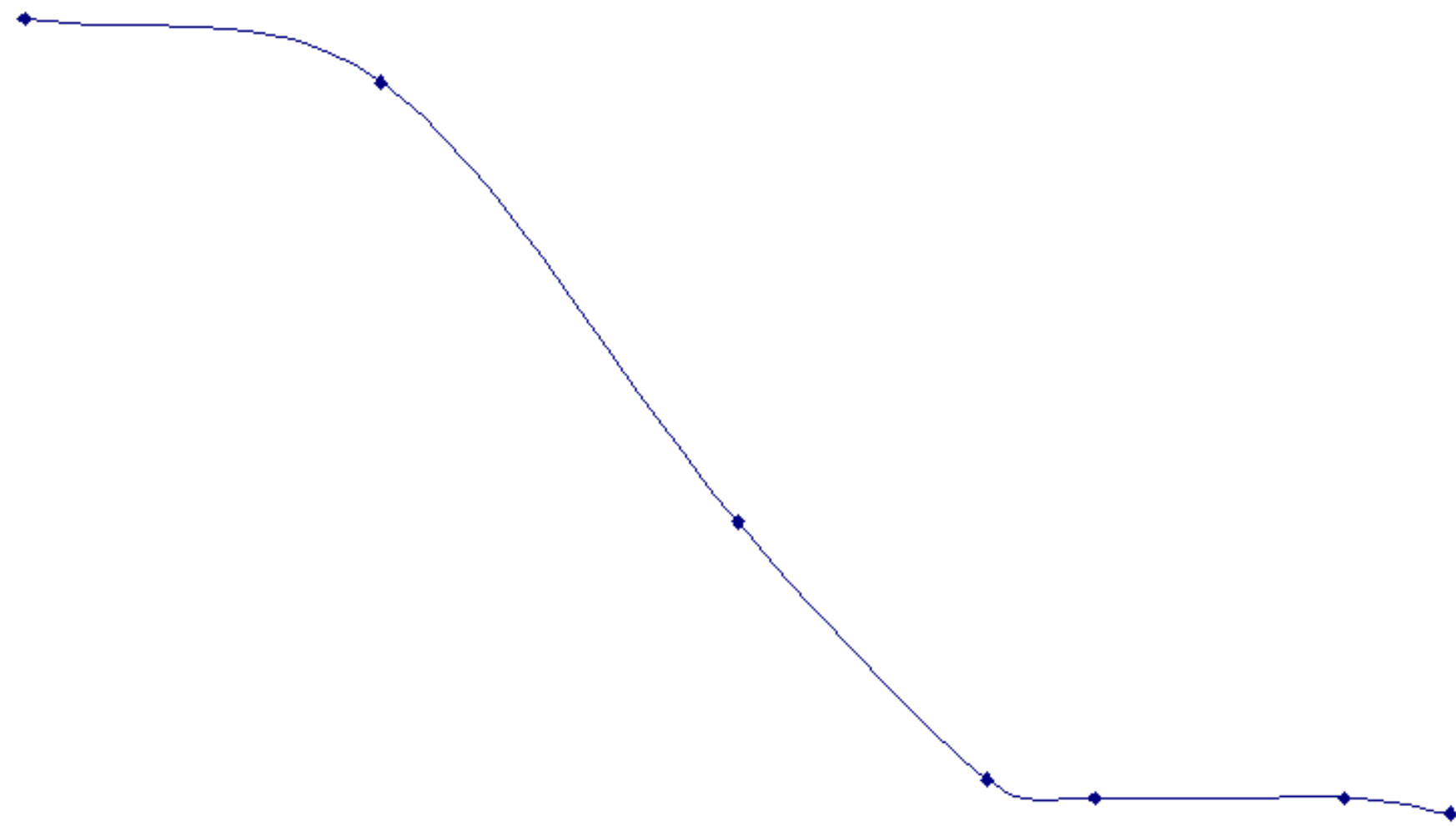




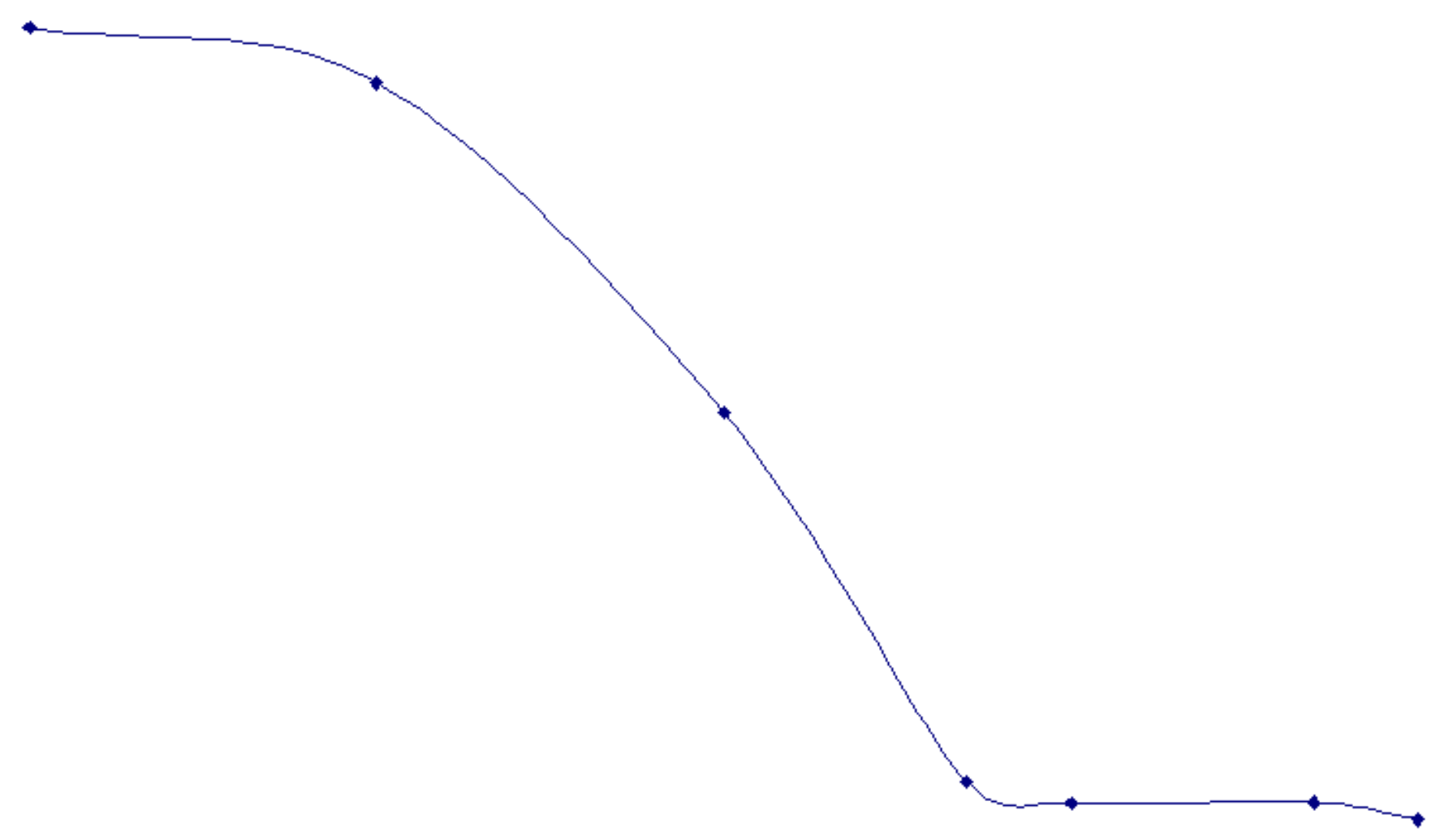



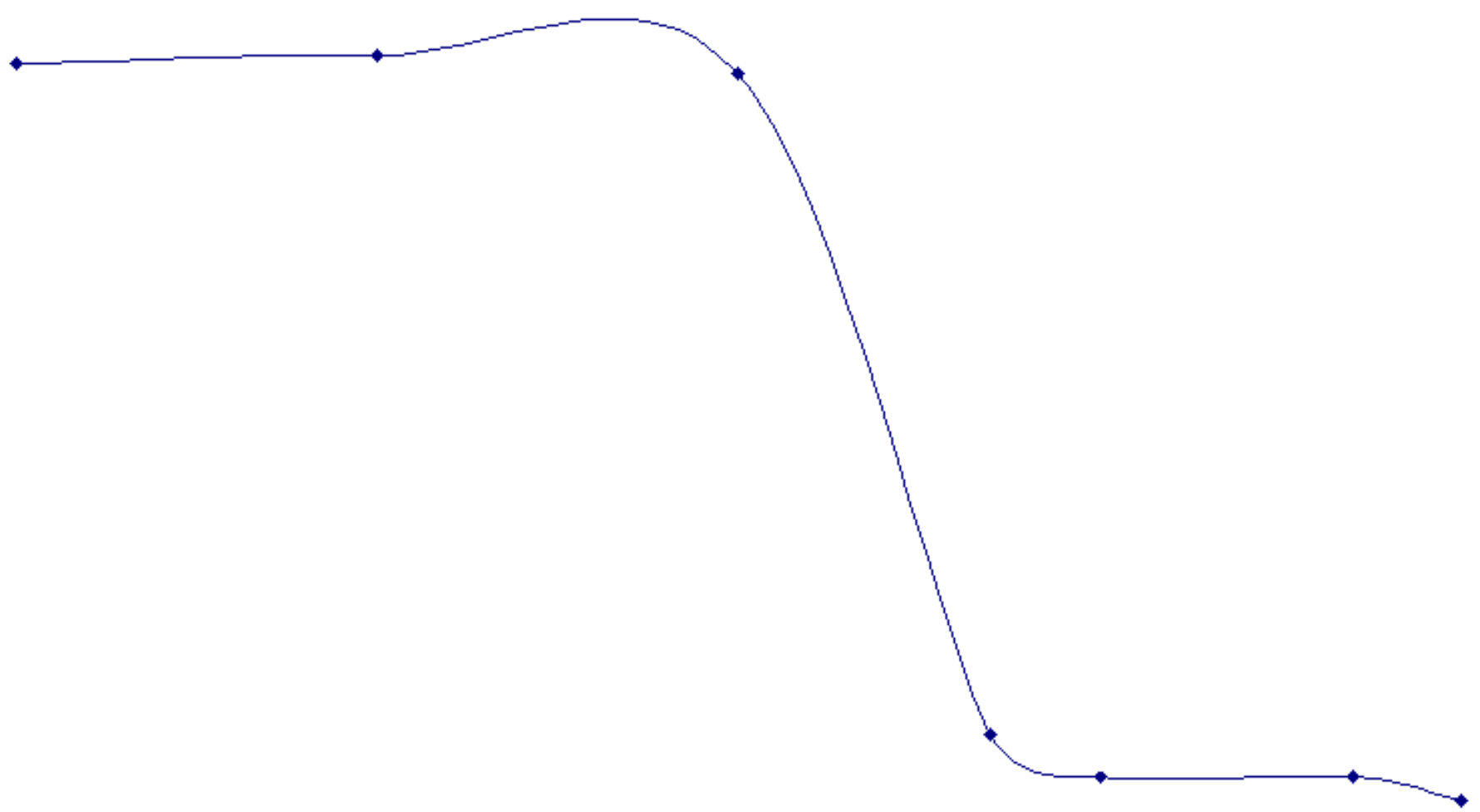